\title{
EMOTIONAL COMMUNICATION IN INSTANT MESSAGING
}

\author{
Afarin Pirzadeh
}

Submitted to the faculty of the University Graduate School

in partial fulfillment of the requirements

for the degree

Doctor of Philosophy

in the School of Informatics and Computing

Indiana University

May 2016 
Accepted by the Graduate Faculty, Indiana University, in partial fulfillment of the requirements for the degree of Doctor of Philosophy.

Davide Bolchini, Ph.D., Chair

Stephen Voida, Ph.D.

Doctoral Committee

Erik Stolterman, Ph.D.

October 29, 2015

Terri Wada, M.F.A. 
(C) 2016

Afarin Pirzadeh 


\section{Dedication}

To my parents. 


\section{Acknowledgements}

Pursuing a Ph.D. project is both an enjoyable and challenging experience and accomplishing this dissertation was not possible without the help of many people who have been patiently and generously supporting me throughout this journey. First of all, I would like to give my sincere thanks to my amazing supervisor, Dr. Mark Pfaff, who offered so much advice and patiently supervised me and guided me in the right direction over the last five years. He gave me the freedom to pursue my idea and encouragement to accomplish my project. Special thanks also go out to Dr. Mattew Palakal and the Indiana University, School of Informatics for believing in me and providing me with a scholarship to continue my Ph.D. program.

I would like to express my sincere gratitude to Dr. Davide Bolchini, who has always been a great support to me throughout the program. I had the most interesting $\mathrm{HCl}$ classes with Dr. Bolchini. His hard work and excitement has always inspired me and I have learned so much from him. Without his continued support, especially throughout my final year, I could not finish my dissertation successfully.

It's a pleasure to express my heartfelt thanks to my committee members, Dr. Erik Stolterman, Mrs. Terri Wada, and Dr. Steve Voida. Thanks go to Dr. Stolterman, who always enlightened my way of thinking and patiently provided me with guidance. I am very grateful for having Mrs. Terri Wada in my committee, who generously helped me through my design process and made sure that I conducted my design sessions properly. Thanks also need to go out to Dr. Stephen Voida for his great feedback on my dissertation, although he was part of my committee for the final year.

My sincere thanks go to Mrs. Karen White, and Dr. Agarwal from Innovation to Enterprise Central (ITEC) and Dr. Kody Varahramyan (Office of Vice Chancellor for Research) to allow me to be a part of the ITEC program and provided their feedback and 
financial support through the design and implementation of Gestchat. I also want to express my sincere gratitude to Dr. Ali Jafari and Cyberlab IUPUI for believing in me and continuously supporting me to implement Gesthchat. I would also like to express my appreciation to all people who offered me their time during my experiments and design sessions.

I am very grateful to my parents (Ahmad and Gita) and brothers (Iman and Peyman), for encouraging me to continue with my higher education, and for supporting me both emotionally and financially whenever it was needed. I cannot thank my parents enough for allowing me to experience this wonderful journey and supporting me through the ups and downs.

Last, but not least, I am greatly indebted to my friends. Their love and support for me without any complaint or regret has enabled me to complete this Ph.D. project. My life in the US would not have been as wonderful as it has been without the company of my friends, Dr. Romisa Rohani, Shiva Ladan, Rob Patterson, Sepideh Ansari, and Sholeh Shahrokhi,. Thank you for being such great and supportive friends. I would also like to thank my great team at Gestchat at Cyberlab: Mohamed Hamdouchi, Yhareli Chamboneth, Jasbir Kaur, Yhareli Chamboneth, David Johnson, Hanlin Li, and Mengyuan Zhao, for all their hard work to implement the first version of Gesthchat. Thanks also go out to Dr. Tao Yang, Dr. Arthur Liu, Dr. Sung, Yamini Karanam, Steve Entezari, and Yuan Jia, for all their support throughout the last five years. I wish you all good luck in your future endeavors. 


\section{Afarin Pirzadeh \\ EMOTIONAL COMMUNICATION IN INSTANT MESSAGING}

Emotional communication is fundamental to everyday interaction. How well emotions are communicated is crucial to interpersonal relationships and individual wellbeing. Emotional communication in instant messaging (IM), however, can be challenging because of the absence of visual and aural nonverbal behaviors. Despite the growing number of technologically-focused solutions for supporting emotional communication in $\mathrm{IM}$, limited design research has been done to study the actual users' behaviors in communicating their emotion in $\mathrm{IM}$ and strategies they use to adapt emotional communication in this medium, with the purpose of establishing design solutions to support users' emotional communication. Connecting several bodies of $\mathrm{HCl}$, design, and communication literature in the context of $\mathrm{IM}$, this dissertation critically examines how users communicate emotion in IM and accordingly establishes user-centered multi-touch gesture based design solutions to support emotional communication in this medium. Understanding how users communicate their emotion in $\mathrm{IM}$, the design issues, and corresponding design solutions help researchers and designers to support the user's emotional needs, resulting in the improvement of emotional communication strategies in IM.

Davide Bolchini, Ph.D., Chair 


\section{Table of Contents}

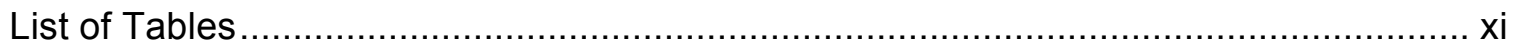

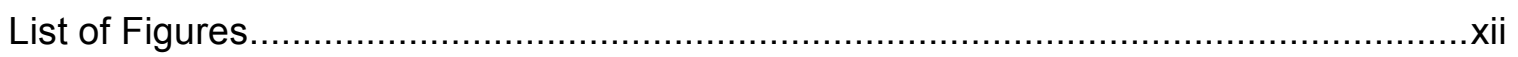

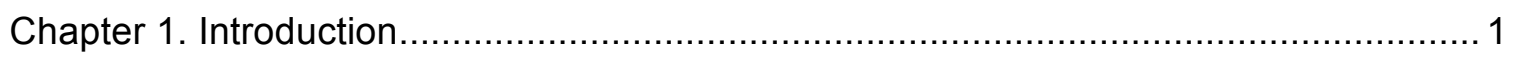

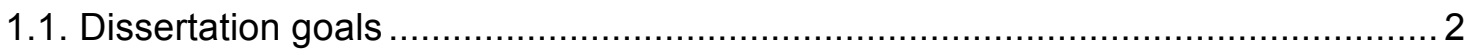

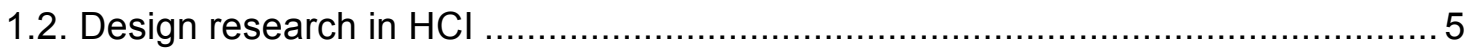

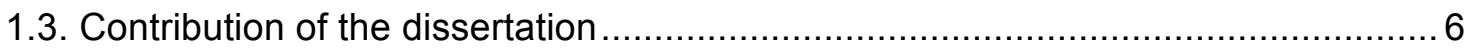

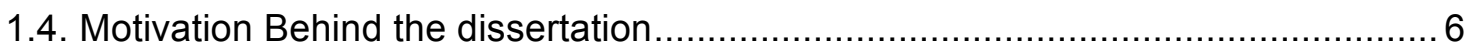

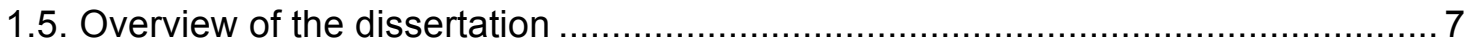

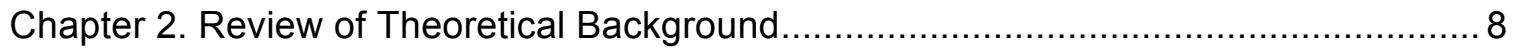

2.1. Text-based computer-mediated communication ......................................... 9

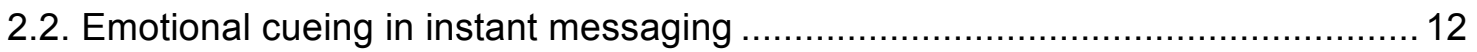

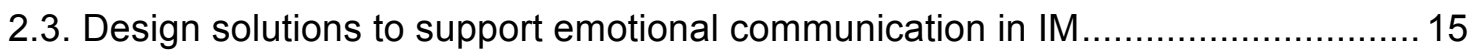

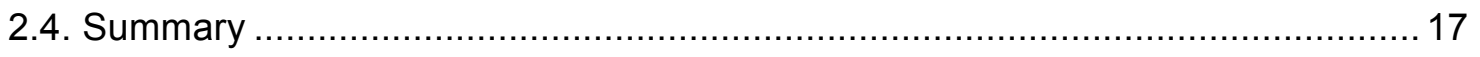

Chapter 3. Study I: Emotion Expression under Stress in Instant Messaging ................. 18

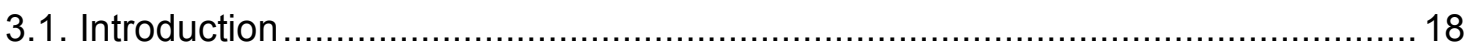

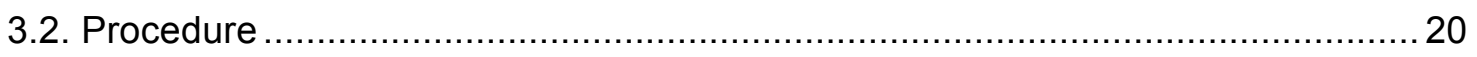

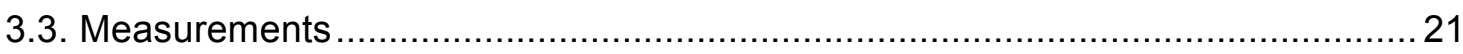

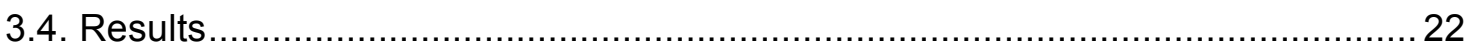

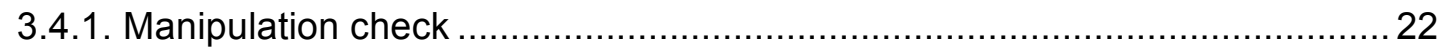

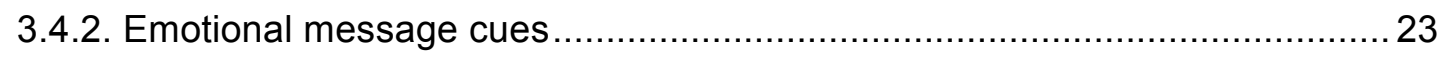

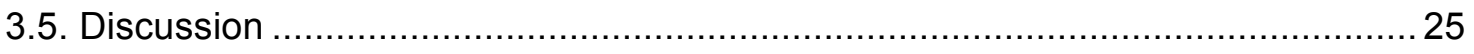

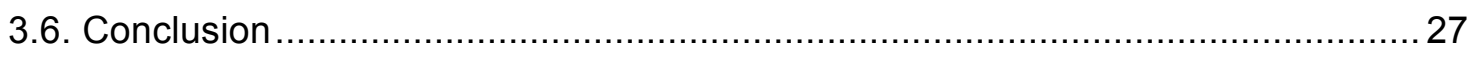

Chapter 4. Study II: Designing Multi-Touch Gestures to Support Emotional

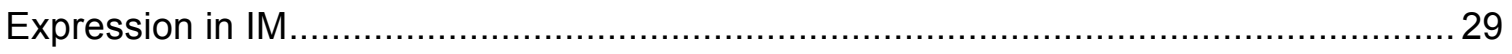

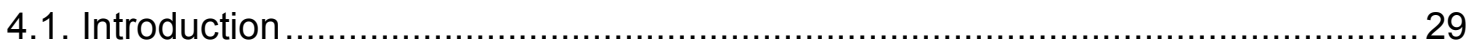




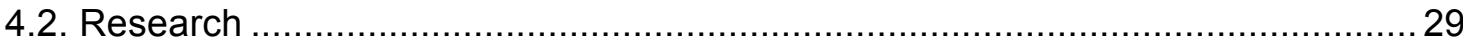

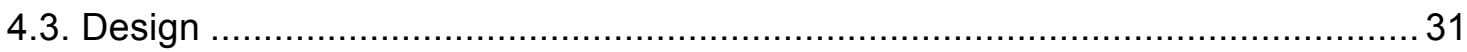

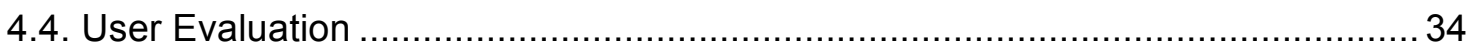

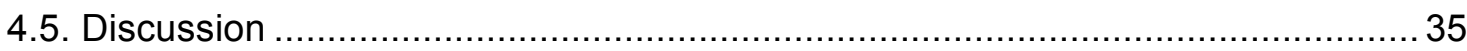

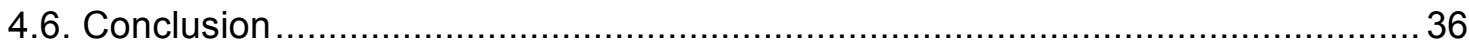

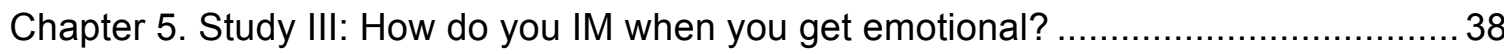

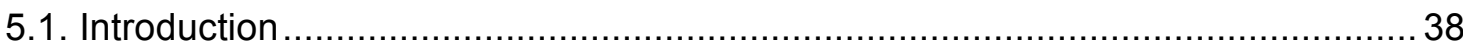

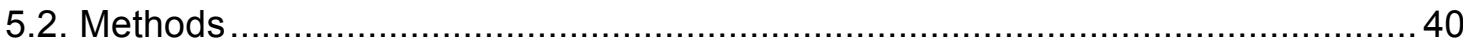

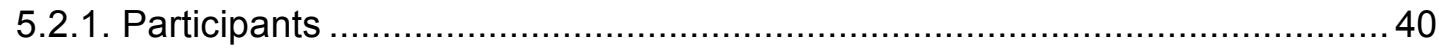

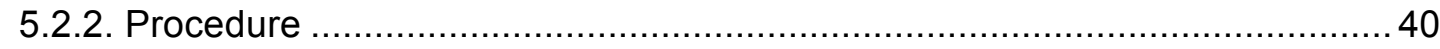

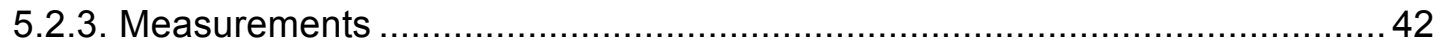

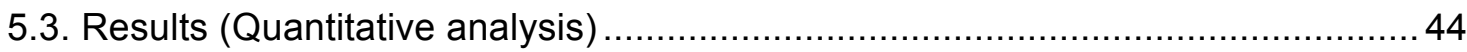

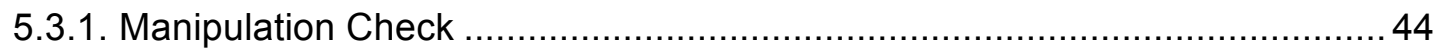

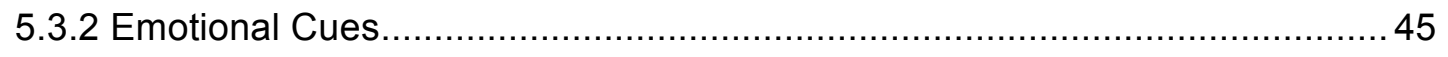

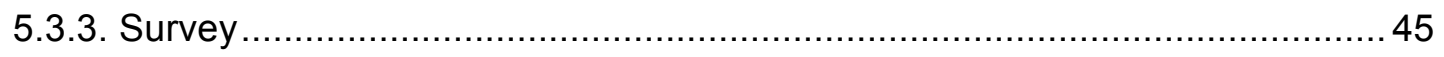

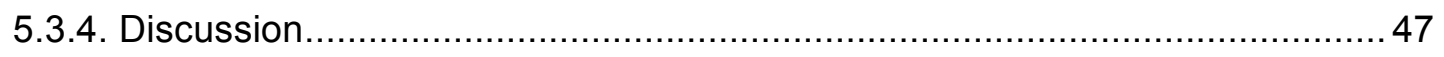

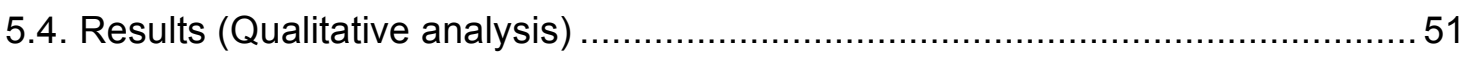

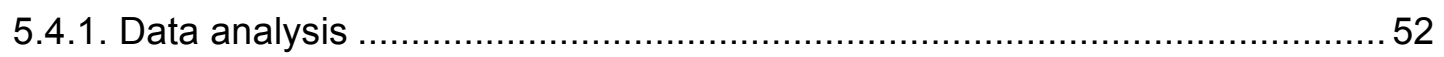

5.4.2. Overall Structure and Main Topics of The Conversations ..........................53

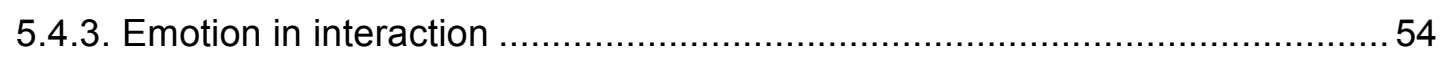

5.4.4. Emotional prosody (capital letters, punctuation, and vocal spelling) .............60

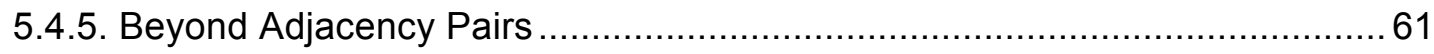

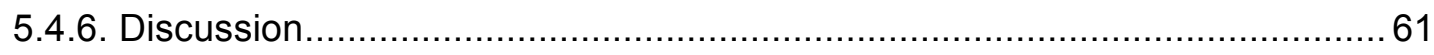

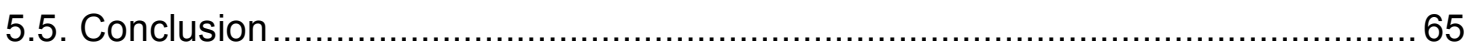

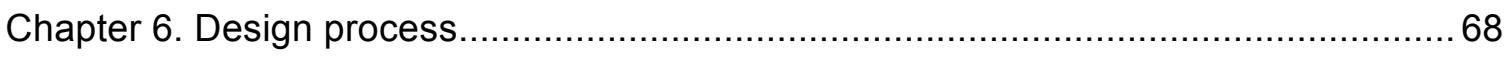

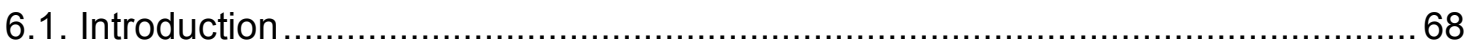




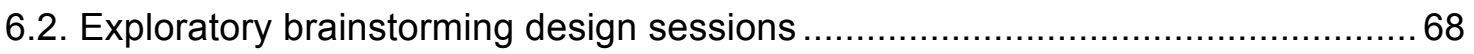

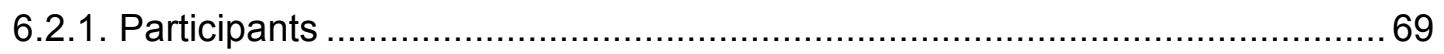

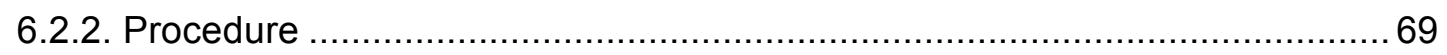

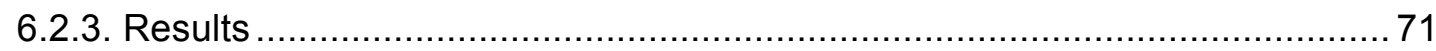

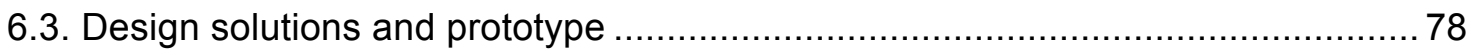

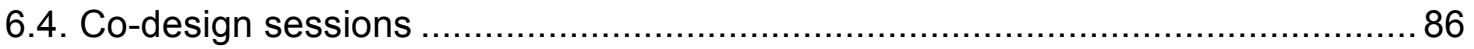

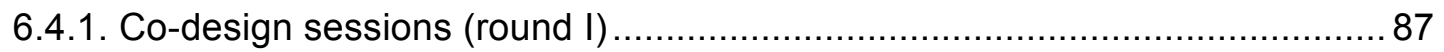

6.4.2. Design Solutions and prototype refinement .......................................... 91

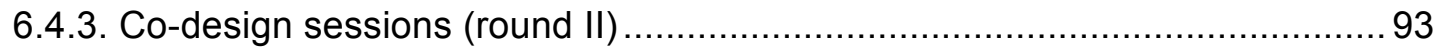

6.5. Design Solutions and prototype refinement............................................... 111

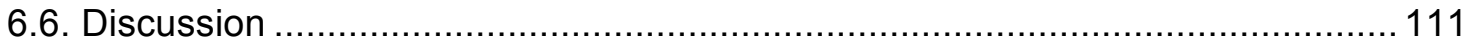

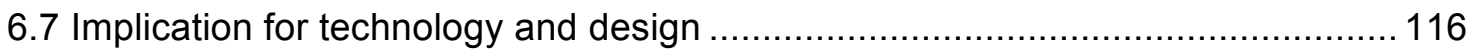

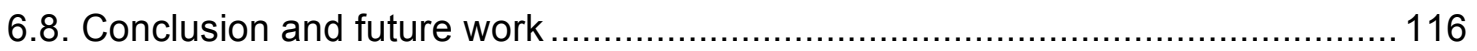

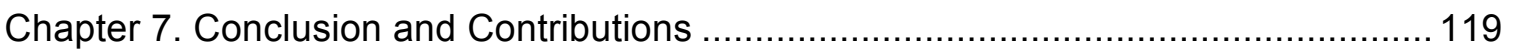

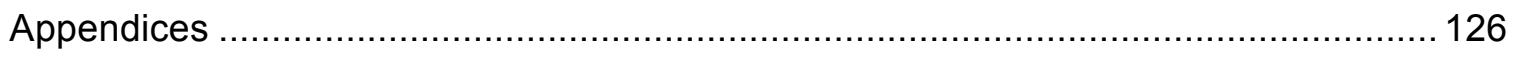

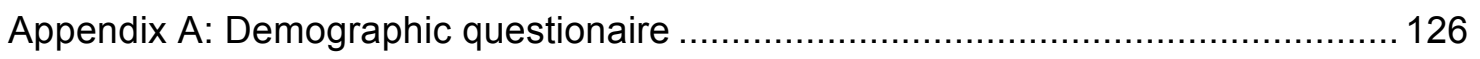

Appendix B: Emotion manipulation check survey ....................................... 127

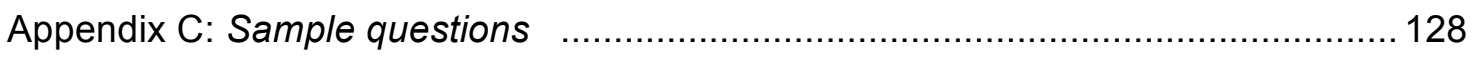

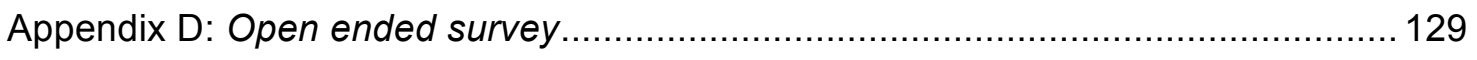

Appendix E: Brainstorming session plan ................................................. 130

Appendix F: Brainstorming session forms ................................................ 132

Appendix G: Emotonal gesture-based communication method patent .................. 136

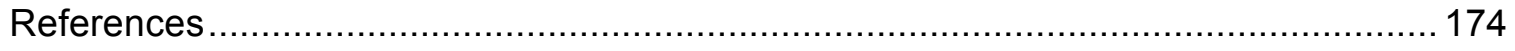

Curriculum Vitae 


\section{List of Tables}

Table 1. Factorial repeated-measures ANOVAs of verbal and nonverbal cues.............24

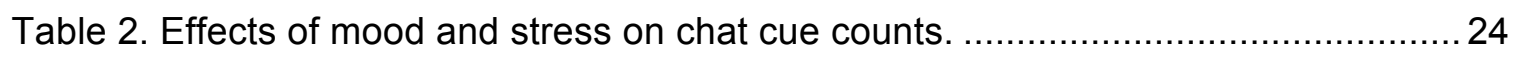

Table 3. Non-parametric correlations $\left(r_{s}\right)$ for trait measures and chat cues...................24

Table 4. Mean emotion levels by condition (SD in parentheses) ............................ 44

Table 5. Mean percentages of verbal and nonverbal cues by condition (SD in

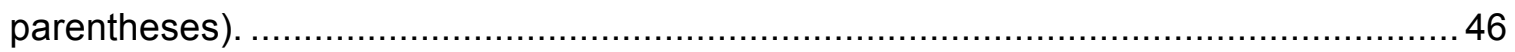

Table 6. Table Agreement cues and emotives in three conditions of sad, happy,

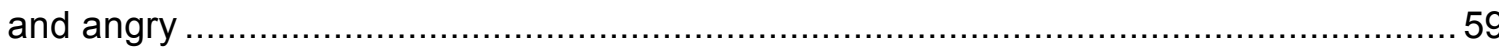

Table 7. List of emotional cues and equivalent multi-touch gestures, body icons,

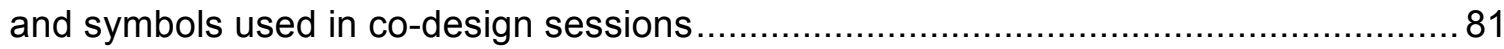




\section{List of Figures}

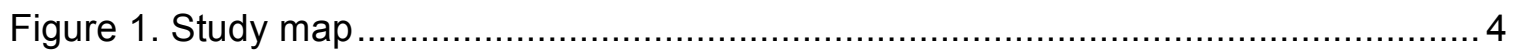

Figure 2. Modified version of Brunswik's lends model (adapted from Scherer,

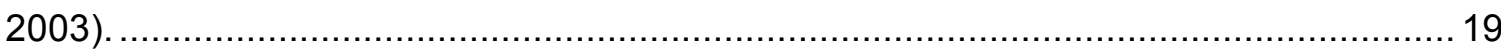

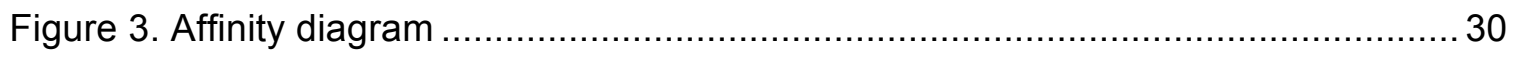

Figure 4. Facial expression and intensity gestures .......................................... 32

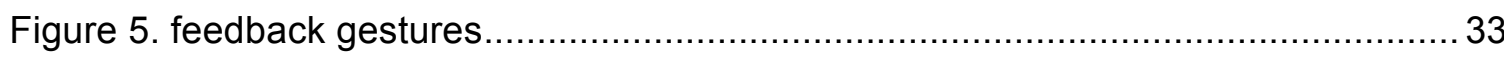

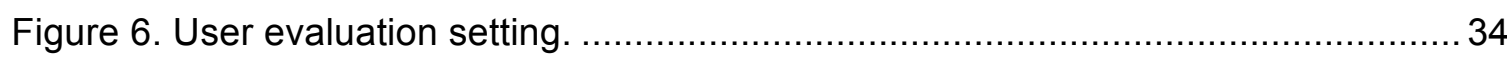

Figure 7. Emotion clusters selected from Russell's (1980) circumplex model, showing valence as the horizontal axis, and activation as the vertical axis.................43

Figure 8. Emotional cues used in emotional communication ...................................55

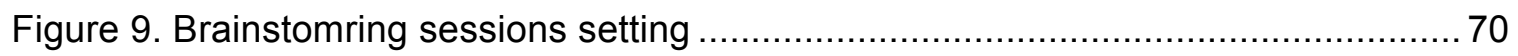

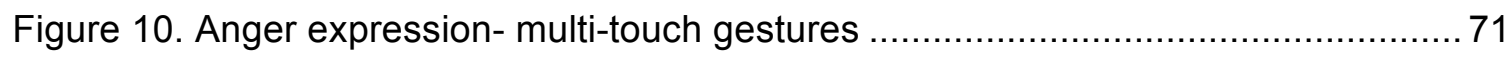

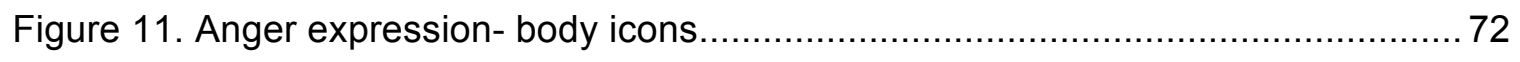

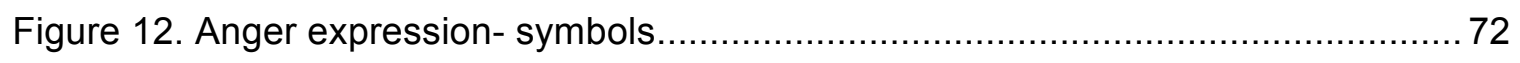

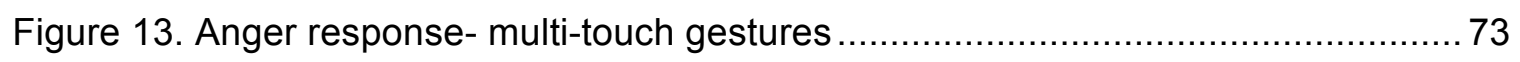

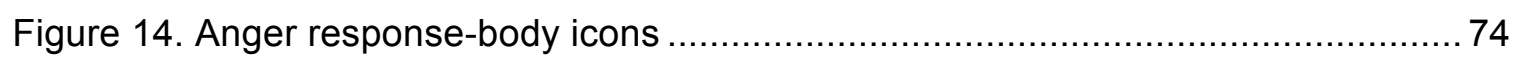

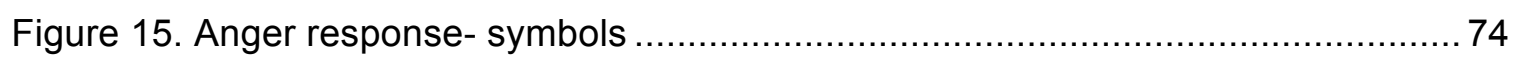

Figure 16. Sad expression- multi-touch gestures............................................. 75

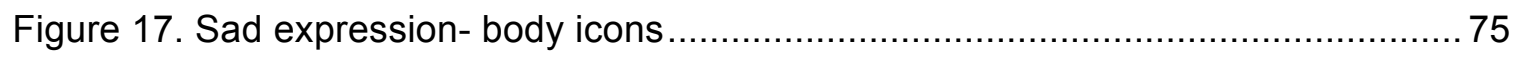

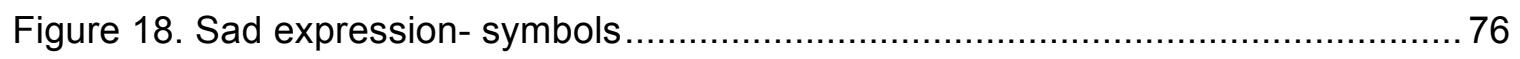

Figure 19. Sad response- multi-touch gestures ............................................ 76

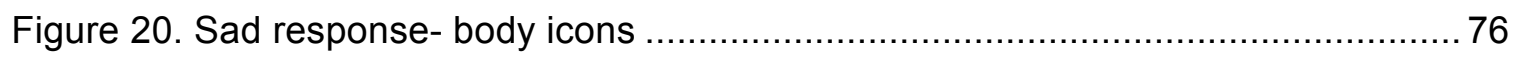

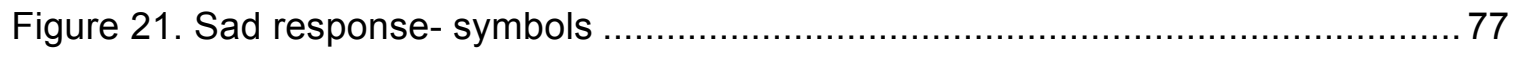

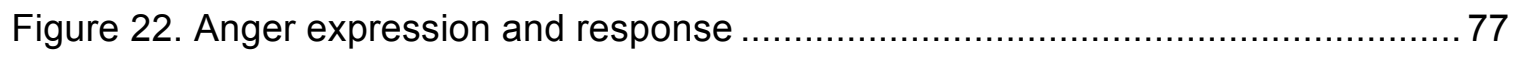

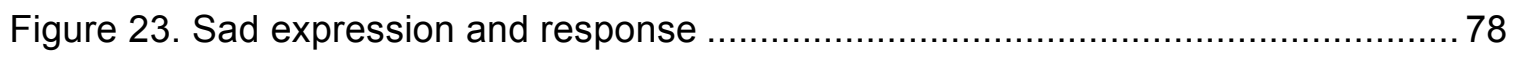




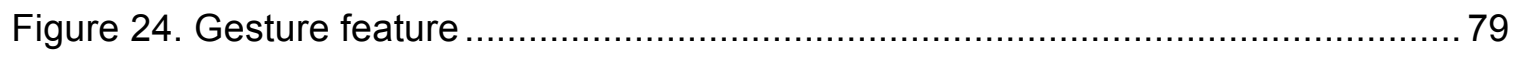

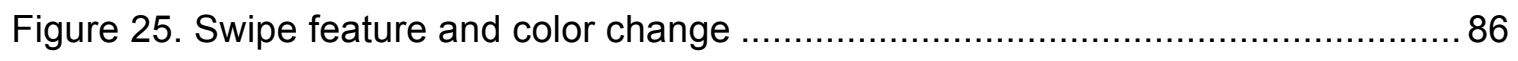

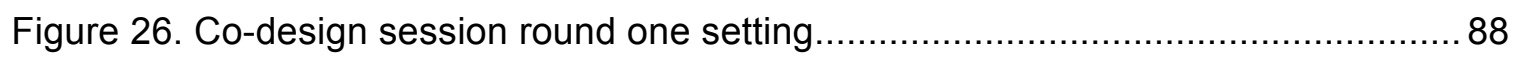

Figure 27. Multi-touch gesture categories created in co-design session round one .......91

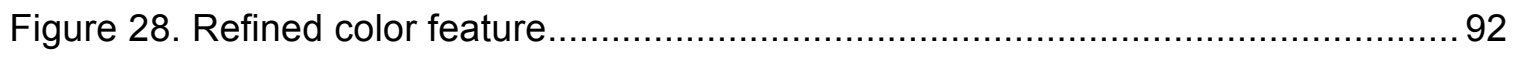

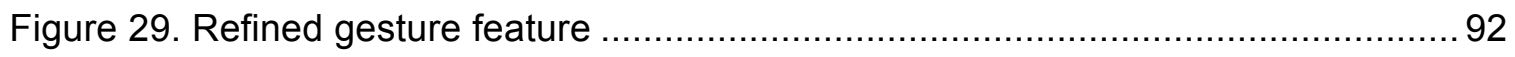

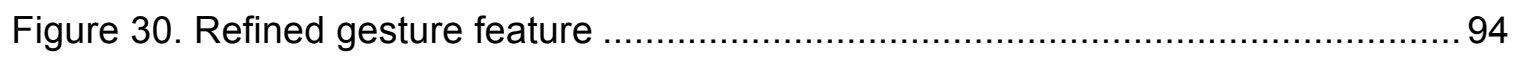

Figure 31. Gestures categories (happy, joking, sad) and sets of body icons,

face icons, symbols, and words related to each category .................................... 95

Figure 32. Gestures categories (indifferent, angry, annoyed, love) and sets of body icons, face icons, symbols, and words related to each category .......................96

Figure 33. Gestures categories (surprised/shocked, questions, confirmation)

and sets of body icons, face icons, symbols, and words related to each category .........97

Figure 34. Combining the results of brainstorming sessions and co-design

sessions with respect to sad and anger response ............................................ 108

Figure 35. Final design of the color and gesture features .................................. 112 
Chapter 1. Introduction

Technology has revolutionized the way people communicate. People use a variety of media to enhance and extend interpersonal communication depending on social, security, or efficiency factors. Communications media, however, affects the quantity and quality of the messages and can change senders' and receivers' behavior and attitudes (Cathcart and Gumpert, 1983). Instant messaging (IM), as one type of synchronous text-based computer-mediated communication (CMC), is not an exception. Despite the advantages of IM communication (e.g. convenience, mobility, and control) over face-to-face, the absence of visual and aural nonverbal behaviors affects communication. There are two lines of research that have studied the effect of IM technology on interpersonal communication.

One line of research showed that the lack of visual and aural nonverbal cues causes some limitations on users' emotional communication in IM (Walther, Loh, and Granka, 2005). IM users sometimes have difficulties expressing their emotions accurately and fail to accurately understand the actual emotion coming from their partners due to the absence of visual and aural nonverbal behaviors. According to Mehrabian (1972), in everyday communications only $7 \%$ of peoples' emotional communication stemmed from the words spoken, whereas $38 \%$ was attributed to verbal tone and $55 \%$ was related to facial expression. Another line of research, however, showed that as IM began growing in everyday life, especially among teenagers and college students (Ramirez and Broneck, 2009), despite the absence of visual and aural nonverbal behaviors, communicators discovered new ways to adjust emotional communication to IM. One of the main theories representing this perspective is Social Information Processing (SIP) (Walther, 1992). SIP argues that people are able to employ different active and passive strategies to convey visual and aural nonverbal behaviors in text-based CMC. Supporting SIP, a growing number of studies have identified different 
strategies (e.g. verbosity, speed of response, or degree of agreement) and diverse textbased emotional cues (e.g. lexical surrogates such as haha, and aha, vocal spelling such as sooo, and weeell) users apply to express their emotions in IM (Hancock, Landrigan, and Silver, 2007; Hancock, Gee, Ciaccio, and Lin, 2008). Connecting these two lines of studies, limited attention has been paid to critically examine how actually users communicate emotion in instant messaging, what challenges they have, and what factors affect emotional communication via this medium.

Emotional communication in IM also provides a variety of new challenges and opportunities for researchers in the area of $\mathrm{HCl}$ and design. A growing number of features, mostly technology focused, such as emoticons, avatars, haptics, and dynamic typography, have been integrated with existing IM systems to support emotional communication (Lo, 2006). This technological-focus leads to developing systems that are novel, but not always able to satisfy user needs in emotional communication via textbased IM.

Connecting different bodies of emotional communication in instant messaging and $\mathrm{HCl} /$ design, user-centered design research (Zimmerman, Forlizzi, and Evenson, 2007 ) is needed first to explore how IM users communicate emotions, and second to establish creative solutions to support emotional communication via this medium.

\subsection{Dissertation goals}

The main goal of this dissertation is to, through design research, critically examine how users communicate their emotions in IM (upfront research) and to establish user-centered design solutions (design process) to comprehensively support emotional communication via IM. The simplicity and variety of multi-touch gestures provide unique opportunities for new forms of human computer interaction. Investigating multi-touch gestures in computer-mediated communication, however, has received limited attention. This project specifically explores multi-touch gesture based design 
solutions and their potential to support emotional communication via instant messaging. A mobile instant messaging application is also designed to communicate and evaluate the design solutions.

Accordingly, this dissertation presents four studies (Figure 1). Three studies were conducted as upfront research to explore how actually people communicate their emotion in instant messaging and potential of multi-touch gesture based design solutions to support emotional communication in this medium. Results of the upfront research were used to inform and inspire the design process and consequently an active process of ideating, iterating, and critiquing potential multi-touch based design solutions to support emotional communication in IM.

The first study was an exploratory study on a preexisting set of 168 chat logs from a previously conducted study on the effects of mood and stress on group communication and performance in NeoCITIES, a multi-player emergency response simulation (Pfaff, 2012). This project presented an initial effort to understand emotional expression in text-based CMC. The main goal of this study was to investigate whether two psychological states (mood and stress) affect the type and quantity of emotional cues users apply in task-focused IM communication. The results confirmed that studying text-based emotional cues and how people actually communicate their emotion in IM merits further study.

The second study was an initial effort to explore the main goal of the dissertation in a small-scale exploratory study. The main purpose of the study was to, through design research, identify text-based cues users employ to express their emotions in IM communication and accordingly explore the potential of multi-touch gesture based design solutions to support emotional expression in this medium. 


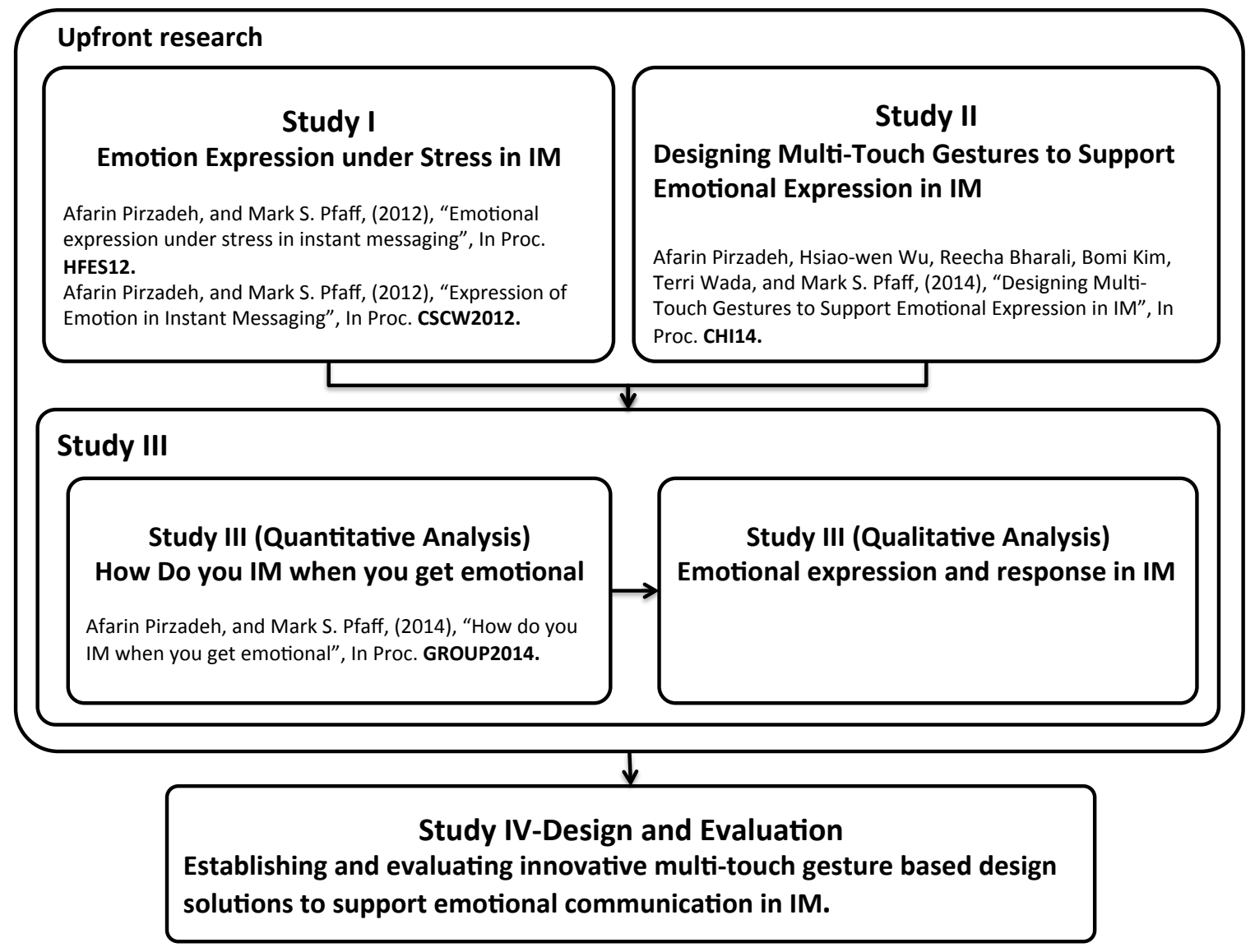

Figure 1. Study map

Based on the insights gained from the first two studies, the third study critically examined how people communicate their emotion in instant messaging through a mixed methodology approach. Participants were involved in informal emotional conversations with their friends in $\mathrm{IM}$, and their conversations were quantitatively and qualitatively analyzed. A quantitative approach was taken to investigate to what extent different emotional states (contentment, angry, happy, and sad) influence the type and proportions of emotional cues individuals apply to communicate their emotions in the context of informal IM conversations. A qualitative approach was also taken through conversation analysis to investigate how emotions were expressed, responded to, and 
developed in IM conversations. Accomplishing this study provided a framework for emotional communication in the context of informal IM conversations and detailed the different strategies and emotional cues users apply to communicate their emotions. It also completed the upfront research foundation for the following final project.

The fourth study was the design process. This study presented an active process of ideating, iterating, and critiquing potential design solutions (with the focus on multitouch gestures) to support emotional communications in mobile instant messaging. A tool (Gestchat) was also designed to communicate the design solutions through this process.

\subsection{Design research in $\mathrm{HCl}$}

This study follows the design research model developed by Zimmerman, Forlizzi, and Evenson (2007). In their model, design research in $\mathrm{HCl}$ (interaction design research) is about engaging in wicked problems found in $\mathrm{HCl}$. Zimmerman et al. (2007) extended Nelson and Stolterman (2003) frame of design as the integration of the real, the true, and the ideal. They argued that the interaction design researcher combines the true knowledge (the current theories and models related to the wicked problem), the how knowledge (technical opportunities presented by engineers), and the real knowledge produced by research. However, the main part of design research is an active process of ideation, iterating, and critiquing potential solutions to engage and continually reframe the wicked problem. The design research focus is not on the outcome (final artifact) to make a design contribution; it implies an inquiry focused on producing a contribution of knowledge. The final output of design research is a concrete problem framing, articulation of the preferred state, and a series of artifact in the format of model, prototype, product and documentation of the design process (Zimmerman, Forlizzi, and Evenson, 2007). 
Design research artifacts are different from design practice artifacts in two aspects. First, the intention behind building design research artifacts is producing knowledge rather than making commercially viable products. Therefore, Zimmerman et al. (2007) suggest researchers who take the design approach ignore or deemphasize the commercial aspects of the artifact such as detailed economics associated with manufacturability and integration of the product into a product. Second, design research artifacts should present a significant invention as the results of novel integration of theory, reality, users need, and technology than refinement or enhancement of the products that already exist.

\subsection{Contribution of the dissertation}

Understanding of users' emotional communication in IM, the design issues, and corresponding design solutions identify gaps in theories and models of emotional communication via this medium. This dissertation also contributes essential insights, theories, and tools to advance human-centered design knowledge at the intersection of $\mathrm{HCl}$ and interpersonal communication and provide the conceptual foundation and a solid, long-term intellectual basis for the creation of substantially improved text-based CMC applications. Such applications will support emotional communication and minimize emotional miscommunication that may occur due to the absence of visual and aural nonverbal behaviors in IM.

\subsection{Motivation Behind the dissertation}

This project was started with the ultimate goal of developing solutions to support IM users communicating their emotions via this medium. The idea for this project developed out of a combination of the researcher's personal experiences and academic interests. It started, on the personal level, with experiencing and witnessing difficulties IM users have in communicating their emotion in IM and how they overcome this challenge. Additionally, the researcher's academic interests drove her to pursue design 
research, prototyping, and developing tools to support individuals and improve the quality of their communication.

\subsection{Overview of the dissertation}

The rest of the chapters in this dissertation are organized as follows. Chapter 2 reviews the theoretical background of this dissertation, which includes a discussion on the computer-mediated communication and a summary of the related works on textbased instant messaging and design solution that have been developed to support emotional communication in instant messaging. Chapter 3 presents the first upfront study on the effect of two psychological states (mood and stress) on the type and quantity of emotional cues users apply in task-focused IM communication. Chapter 4 explains the second upfront study on exploring the potential of multi-touch based gestures to support emotional communication in instant messaging. It also documents lessons learned to design the following upfront study. Chapter 5 presents the third study on exploring how actually people communicate their emotion in $\mathrm{IM}$, including both quantitative and qualitative analysis of data. The quantitative analysis investigated the effect of four emotional states (relaxed, angry, happy, sad) on the type and quantity of emotion-related cues used during informal conversations between college friends in IM, while the qualitative analysis explored how actually people expressed and responded emotion in IM, through conversation analysis. Chapter 6 presents the design process of ideating and evaluation of multi-touch gesture based design solutions to support emotional communication via IM. This chapter also summarizes the design process of Gestchat, a mobile text messaging application that supports users emotional communication. Finally, Chapter 7 discusses conclusion, overall contribution of the dissertation, and possible future research directions. 
Chapter 2. Review of Theoretical Background

Common to all models of communication is the idea that information is transferred from sender to receiver. This information is encoded by the sender and has to be decoded by the receiver to understand the message (Harrison, 1974). Whittaker (2003) categorized communication phenomena in two main categories of cognitive and social/emotional cueing, which refer to the exchange of cognitive and social/emotional information through verbal (words) and nonverbal (wordless) cues in communication. Nonverbal cues include any body movement, facial expression, eye contact, vocal cues, haptic, physical appearance and artifacts, proxemics, and time.

Nonverbal behaviors play an important role in difference aspects of cognitive cueing such as turn-taking, initiating impromptu conversation (availability), referencing and shared attention (shared environment), and interactivity. Nonverbal behaviors also mediate different aspects of social/emotional cueing such as emotional content, negotiation, and social processes (e.g. participation and acceptance):

- Emotional content: Emotional content refers to information about communicators' affective and attitudinal state, along with back-channel feedback they use in communication. Visible and aural behaviors such as facial expression, gaze, body movement, gestures, and vocal cues contribute to the communication of affective and attitude state information. Facial expression plays an important role in communicating affective state of individuals and their attitudes. Ekman (1982) showed seven distinct emotional states, happiness, sadness, surprise, anger, disgust, fear, and interest can be recognized from facial expression. Gaze is also another visible indicator that shows the emotional states of conversational participants. When speakers are more persuasive or assertive they may gaze at the listener's face more (Kleinke, 1986). There are also a high 
number of studies on the relationships between body movement and gestures, and emotional expression. For example arm movement with different velocity, acceleration, and displacement usually are made with the intention to joy, sadness, and anger expression (Sawada, Suda, Ishii; 2003).

- Negotiation and deadlock: This aspect of social cueing refers to the engagement in negotiation and how it leads to a deadlock or success of the stronger participant in negotiation.

- Participation and acceptance: The amount that people talk and get involved in conversations and the pattern of acceptance of others contribution is another category of social cuing that is partly related to the perceived status of the individuals within the group.

Lack of nonverbal cues in text-based computer-mediated communication affects cognitive and social cueing via this medium. Since the main focus of this dissertation is on emotional communication in $\mathrm{IM}$, the following section presents a short history of textbased computer-mediated communication and explains a variety of studies in the CMC area that explore the impact of this type of communication on cognitive and social/emotional cueing, with regard to the lack of nonverbal cues. It also describes design solutions that have been developed with the purpose of supporting emotional communication via this medium.

\subsection{Text-based computer-mediated communication}

$\mathrm{CMC}$ arose from linking computers to one another in networks, and consequently operators found that in addition to sharing data, they also could send simple messages to one another. From there, CMC grew from simple message dispatch systems to multimedia group communication applications (Walther, 1996). Early research on CMC focused on different communication modalities and mainly argued that, regardless of the 
task, the more similar CMC is to face-to-face communication, the more efficient the communication will be. This is referred to as the bandwidth hypothesis (Whittaker, 2003), which itself is rooted in information theory (Shannon \& Weaver, 1949).

The main cause of the inefficiency of text-based CMC in earlier research was explained by the absence of visual and aural nonverbal cues, which convey cognitive, social, and emotional information in face-to-face communication (Kiesler, Siegel, \& McGuire, 1984, Culnan \& Markus 1987). Culnan and Markus (1987) refer to this as the cues-filtered-out perspective.

Further research on $\mathrm{CMC}$ began to reject the bandwidth hypothesis, leading to a wave of studies examining the exchange of cognitive information. Many studies (e.g. Chapanis, Ochsman, Parrish \& Weeks, 1972; Chapanis, Parrish, Ochsman \& Weeks, 1977) demonstrated that face-to-face interaction does not necessarily increase the efficiency of communication. In fact, several studies showed that CMC could be more efficient than face-to-face in some task-oriented communication, including cognitive problem solving tasks and team coordination (Reid, 1977). Lack of visual and aural nonverbal cues, however, can still affect different aspects of cognitive cuing such as turn taking, availability, referencing and shared attention, and interactivity. For example, based on Isaacs et al. (1997) information about the physical presence, current activities or movement of other people, which are lacking in text-based CMC, affect different aspects of impromptu conversations (Isaacs, Whittaker, Frohlich \& O'Conaill, 1997). Garcia \& Jacob (1999) showed how non-interactive technologies turn linear turn-taking in oral conversation to a multidimensional and non-linear sequentiality. Oviatt and Cohen (1991) explained how the lack of interactivity leads to more elaboration and redundancy in text-based CMC.

In 1976, researchers began to study the effect of text-based CMC on social cuing. Despite the effectiveness of text-based CMC for cognitive tasks, different studies named 
this type of communication as antisocial, impersonal, and unable to convey social and emotional cues (Walther, 1996; Walther, Anderson, \& Park, 1994). One of the main theories on social cuing in text-based CMC is social presence theory (Short, Williams, \& Christie, 1976). Social presence theory characterizes technological differences in terms of how they present a sense of other communication participants' goals, attitudes, and motives. Face-to-face communication and video/speech methods (e.g. Skype ${ }^{\mathrm{TM}}$ ) provide rich visual interpersonal information afforded by gaze and facial expressions, making them high on the scale of social presence, with text-based communication at the low end of this scale. According to this theory, technologies that fail to communicate social presence, such as text-based $\mathrm{CMC}$, will negatively affect social and emotional communication.

Over time, text-based CMC flourished in additional contexts such as social chat groups and online forums. Researchers began finding that text-based CMC could indeed be more interpersonal than previously thought, as communicators discovered new ways to express emotional and social information in text. One of the main theories representing this perspective is Social Information Processing (SIP; Walther, 1992). SIP argues that people are able to employ different active and passive strategies to convey visual and aural nonverbal behaviors in text-based CMC. In addition, it asserts that communicators can develop social relationships via text-based CMC as well as through face-to-face interaction (Walther, 1996). This perspective acknowledges that the rate of social information cues exchange may be less in text-based CMC compared to face-toface communication due to the absence of explicit and visible nonverbal cues, but the interpersonal relationship formed over text-based CMC may eventually exhibit the same qualities of face-to-face interaction (Walther, 1993).

Compared to prior views of text-based $\mathrm{CMC}$ as an impersonal mode of communication, these studies demonstrated that this type of communication could be 
highly interpersonal. Text-based CMC can indeed convey emotional and social information when desired and appropriate, or it can remain impersonal and task-oriented when that is the desired interactive setting. For example, to conduct brainstorming, group coordination, or decision-making through text-based CMC, strategies such as time pressure, reduced periods for discussion, anonymous interaction, turn taking, or floor sharing techniques can be applied to encourage task-oriented communication. Although the above strategies might be helpful to impersonalize text-based CMC, they may also affect task outcomes if social or emotional information is necessary to reach the best results. Hence, careful judgment should be applied when selecting communication tools for a given task.

To conclude, computer mediation alone does not determine whether a communication is impersonal or interpersonal. The specific technical implementation of a CMC system provides a basis for communicators to set their communication tone as preferred. Impersonal CMC may be desired when users wish to capitalize on its strengths for task-focused communication. On the other hand, CMC can also be a highly interpersonal means of communication when users have the time and technological affordances to build and express their emotions while exchanging information (Whittaker, 2003). Walther (1992) went even further and offered a new perspective explaining that at high levels of intimacy, emotions and affinity via CMC could exceed face-to-face interpersonal communication.

\subsection{Emotional cueing in instant messaging}

Supporting SIP theory, one growing line of research on text-based CMC (e.g. Walther, Loh, and Granka, 2005; Hancock, Landrigan, and Silver, 2007; Hancock, Gee, Ciaccio, and Lin, 2008) explored different strategies and text-based emotional cues people rely upon to overcome the loss of visual and aural nonverbal cues in emotional/social cuing in this channel of communication. Boonthanom (2004) described 
two main categories of text-based emotional cues, verbal and nonverbal, that users apply to express their emotions in text-based CMC. Verbal cues are a linguistic mechanism including emotion words (e.g. happy, angry) and linguistic markers (e.g. I want to thank him a thousand times). Linguistic markers express emotion although they do not contain emotion words. Nonverbal or paralinguistic cues in text-based CMC express meanings normally transferred by nonverbal physical behaviors, like tone of voice or body gesture. These include five types: vocal spelling (altering spelling to mimic a specific vocal inflection, e.g. weeeell, soooo), lexical surrogates (textual representations of vocal sounds that are not words, e.g. uh huh, haha), spatial arrays/emoticons (pictographs constructed from punctuation and letters, e.g. :-( for a sad face, or :-D to indicate laughing), manipulation of grammatical markers (alterations of the presentation of words, e.g. all capital letters, strings of periods or commas), and minus features (deliberate or inadvertent neglect of conventional formatting elements, e.g. lack of capitalization or paragraphing).

Hancock, Landrigan, and Silver (2007) suggested that individuals adapt their emotion expression to a text-based communication environment by developing four strategies (degree of agreement, negative affect terms, punctuation, speed of response and verbosity). Hancock, Gee, Ciaccio, and Lin (2008) also showed that people in a negative mood produced fewer words and used more negative terms.

Despite the growing number of quantitative studies that identified what emotional cues IM users apply to express different emotions via IM, limited attention has been paid on how users build and communicate emotion throughout the IM conversation using different emotional cues. This area, however, has been explored in face-to-face communication. A high number of studies on face-to-face communication investigated emotion as an emergent theme in conversation analytic research (Sindell \& Stivers, 2012). 
Conversation analysis research on emotion arose as a field of study in the1960s through Erving Goffman's essay “Fun in Games” (1961) that set a foundation for further studies on emotion in interaction (Jefferson, Sacks, \& Schegloff, 1987). Conversation analysis on emotion treats emotion as a social display and mainly depicts how emotion is communicated in specific situations in talk-in-interaction (Sindell \& Stivers, 2012). The early work on emotion in interaction in face-to-face communication through conversation analysis (Guffman, 1961) showed that emotion regulation is a constant task for the participants of a conversation. Participants engage in self-disclosure of their individual feelings according to their interpretation of the situation at hand throughout the conversation. However, "flooding out" may also happen where participants allow themselves to burst into laugher and crying, or become open to shame and anger. Couper-Kuhlen's (2009) research on displays of disappointment conceptualizes affect as "a context-dependent interpretation based on lexical and prosodic cues in specifiable sequential locations." She concentrates on tone of voice as a particular modality of producing "Oh" following a rejection of a request or proposal. Overall she argues how emotional displays can be relevant to the proceeding conversation and how people orient to an underlying affective structure in conversation.

Emotion is also studied as a co-constructed interactional resource. Wilkinson and Kitzinger (2006) show how surprise is interactionally achieved and how displays of surprise in interaction pursue different goals such as signaling the affective stance of the interlocutors and finding a shared understanding of the local moral order. Ruusuvuori and Peräkylä (2009) also described how the interplay of different modalities (facial expression alongside spoken interaction) in managing emotion in interaction could be studied using conversation analysis. In instant messaging communication, however, this in-depth qualitative approach received limited empirical scrutiny. 


\subsection{Design solutions to support emotional communication in IM}

The dramatically increasing use of text-based chat for interpersonal communication in everyday life also led to development of diverse design solutions to improve emotional and social communication via this medium (Lo, 2006). The following are some of the solutions designed to support users in emotional and social communication in IM:

- Emoticons: One of the most used and researched forms of design strategies to support nonverbal communication in IM is the use of graphic emoticons (Riva, 2002). Emoticons were defined as iconic forms or visual cues to substitute for the absence of nonverbal cues such as gestures and facial expression and indicate the sender' mood or feeling (Gajadhar \& Green, 2005).

- Avatars: Using avatars is another design strategy that has been integrated into IM environment in several studies. Kaliouby and Robinson (2004) presented FAIM as an instant messaging application that used automated facial expression recognition and displayed the emotion to the chat partner by an expressive avatar. Neviarouskaya, et al. (2007) also used avatars to convey emotion in IM chat. They developed AffectIM in which the emotion of the user is detected by analyzing the emotional content of the text and automatically transferred to an avatar with inferred emotion.

- Haptics: Integrating tactile technology into IM space is another strategy to support nonverbal communication. Haptic IM uses waveforms with different frequencies, amplitudes, and durations, to which emotional meaning can be attached and transferred by haptic devices such as joysticks and touchpad (Rovers and Essen, 2004). Brave and Dahley 
(1997) introduced InTouch, as a conceptual prototype, using haptic technology by suggesting a shared object that could be manipulated by both IM users. Rovers and Essen (2004) presented the concept of hapticons as programmed predefined waveforms used to communicate emotions, in a similar manner as emoticons, through haptic devices; for example, a smiley face as a waveform with moderate frequency and growing amplitude, or frowny face with high frequency and amplitude in abrupt pulses. The ContactIM application developed by Oakley and O'Modhrain (2003) is another example of integrating haptic information into IM environment. In their application users have a virtual field beside the IM window and can toss a ball between each other. The act of throwing a virtual ball may convey different degrees of emotion; for example, lightly thrown ball as playful and happy gestures compared to a fast thrown ball as disagreement and anger.

- Dynamic Typography: Kinetic typography is another design strategy that supports emotional communication in IM. It is explained as a real-time modification of text, such as font, color, and size in IM that can be used to communicate users' emotion (Yeo, 2008). Wang et al. (2004) developed an IM application that used galvanic skin response (GSR) as an indicator of user's arousal and animated text by changing color, font and size.

Gestures represent a form of nonverbal communication in face-to-face communications that is making an extensive contribution to the field of $\mathrm{HCl}$ (Karam and Schraefel, 2005). For example, in desktop and mobile computing different gestures have been developed as an alternative to the mouse and keyboard interactions for manipulating objects or annotating documents (lannizzotto, Villari, and Vita, 2001). Such 
gestures may fill the gap in emotional communication in IM. However using gestures in this area has received limited attention.

\subsection{Summary}

To summarize, previous studies supported SIP (e.g. Walther, Loh, and Granka, 2005; Hancock, Landrigan, and Silver, 2007; Hancock, Gee, Ciaccio, and Lin, 2008) explored different strategies and text-based emotional cues people rely upon to overcome the loss of visual and aural nonverbal cues in emotional/social cuing in this channel of communication. However, limited attention has been paid to examine how users apply emotional cues to express their emotion, and what challenges they still encounter to express their emotion via this medium. Exploring different challenges users encounter in emotional communication in IM may support social presence theory (Short, Williams, \& Christie, 1976), in which the lack of visual and verbal cues negatively affect emotional communication.

Therefore, with respect to two main bodies of literature on emotional communication in text-based CMC, social presence theory (Short, Williams, \& Christie, 1976) and social information processing (SIP) (Walther, 1992), the main goal of this dissertation is, through design research, to study emotional cuing in instant messaging in different emotional states of happy, sad, and angry. This research leverages the strengths of both quantitative and qualitative approaches to examine what and how emotional cues are used by IM users, to express their emotion via this medium. This dissertation also derives novel user-centered design solutions to comprehensively support emotional communication via IM, in particular through multi-touch gesture-based interaction. The following chapters present three upfront research studies and design processes that were conducted to reach the main goal of the dissertation. 
Chapter 3. Study I: Emotion Expression under Stress in Instant Messaging

\subsection{Introduction}

As an exploratory study, a preexisting set of 168 chat logs were analyzed from a previously conducted study on the effects of mood and stress on group communication and performance in NeoCITIES, a multi-player emergency response simulation (Pfaff, 2012). The main purpose of this study was to explore the influence of two psychological states (mood and stress) and a range of personality traits on the type and quantity of emotion-related cues in IM. Therefore, controlling for personality traits of the participants, the relationships between induced psychological states (mood and stress) and emotional cues participants used in IM were investigated in the context of the NeoCITIES team decision-making task.

This study aimed to extend the findings of previous studies on emotional expression in IM (e.g. Boonthanom, 2004; Hancock et al., 2007; Hancock et al., 2008) in three aspects. The first aspect was studying the combined effects of mood and stress on emotion communication in IM. A focus on task-related stress (in this case, time pressure) afforded an opportunity to better understand emotional cues in CMC in more missioncritical contexts, such as command and control, as opposed to casual or day-to-day IM. Although stress is associated with emotion, stress is a distinct cognitive and affective process that can be manipulated and measured independently of mood (Pfaff \& McNeese, 2010). Johnstone \& Scherer (2000) describe how stress reliably produces changes in speech and vocal communication of emotion, reporting that intensity and fundamental frequency are significantly higher in vocal communication of emotion under stress. Analogous cues may be evident in IM.

The second aspect was applying the lens model (Brunswik, 1956) as the theoretical framework for communication of emotion in synchronous text-based CMC. This model was frequently used for research on emotional communication (e.g. Aronoff, 
Woike, \& Hyman, 1992; Scherer, 2003). The results from Boonthanom's (2004) study on asynchronous text-based CMC confirmed the theoretical validity of the Brunswik's lens model in the field of text-based CMC. The modified version of the lens model applied in this study is shown in Figure 2. This approach models the process of encoding (expression) and decoding (impression) of emotional communication. All communication, including IM, is embedded in a framework of culture, social rules, situational context, and individual differences.

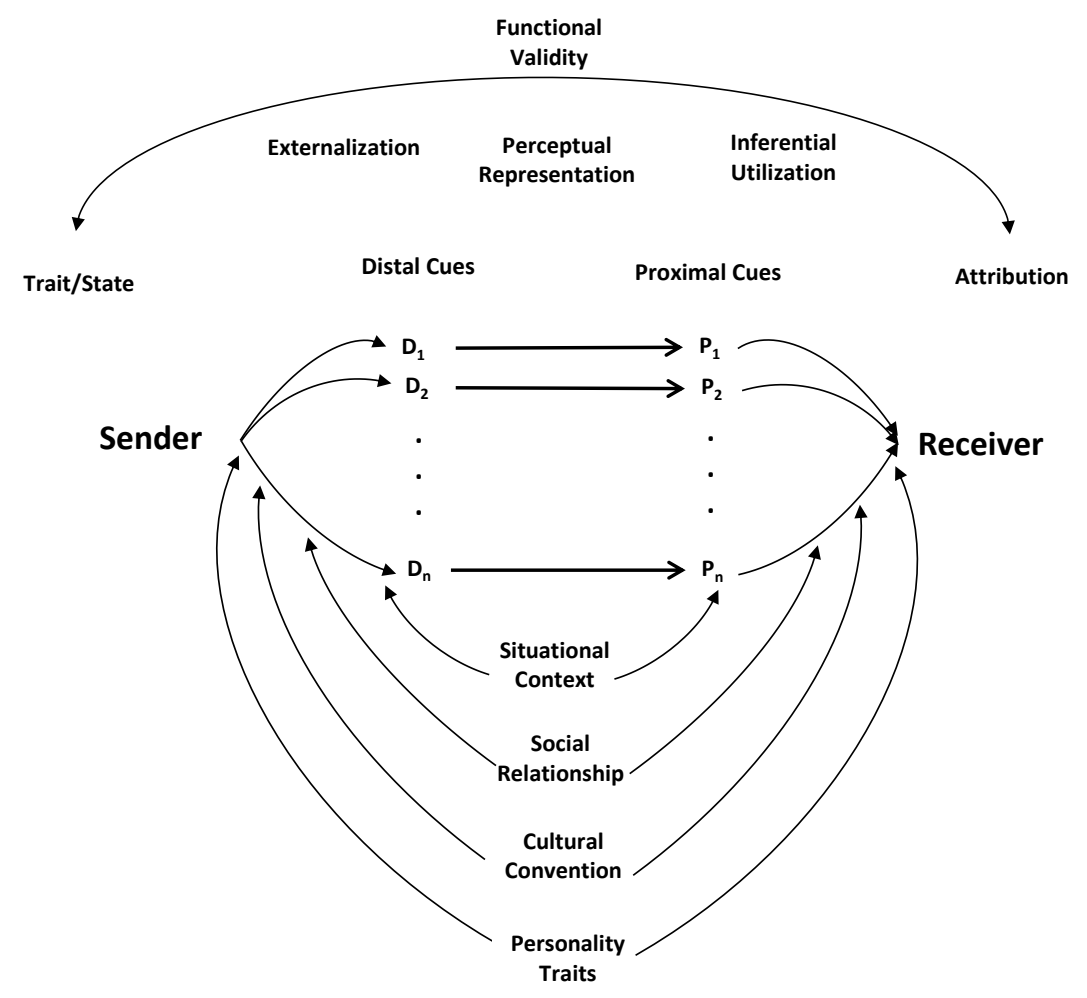

Figure 2. Modified version of Brunswik's lends model (adapted from Scherer, 2003).

The third aspect was investigating the relationship between Boonthanom's (2004) emotional cues in text-based $\mathrm{CMC}$ and personality traits of the individuals in the conversation. Many studies have explored the effect of personality traits on nonverbal 
behaviors in face-to-face communication. For example, Scherer (1978) demonstrated that extraversion could be directly recognized from the vocal cues; such individuals tend to speak with a louder and possibly more nasal voice. This is relatively unexplored in CMC.

Therefore, this study measured emotional communication under stress in IM as a way of understanding the relationship between two particular nodes in the lens model: the psychological traits and states of the encoder, and indicators and cues appearing as a result in IM in the context of situational stress. Specifically, this study measured the influence of two psychological states (mood and stress) and a range of personality traits on the use of emotion-related cues.

\subsection{Procedure}

This was an exploratory study using preexisting data to detect possible patterns or trends meriting more focused future research. 168 chat conversations were analyzed from a previously conducted study on the effects of mood and stress on group communication and performance in NeoCITIES, a multi-player emergency response simulation (Pfaff, 2012). Forty-two participants, ranging from 19 to 30 years $(M=21.1$ years, $S D=1.98$ ), were drawn from a pool of undergraduate students enrolled in a junior-level human-computer interaction course. Thirty-eight were male and four were female.

Participants were randomly assigned to seven teams of six members each. Each team played the NeoCITIES simulation four times over the course of two weeks in a within-subjects full-factorial design with two levels of mood (happy/sad) and two levels of stress (low/high time pressure). Mood was manipulated using pre-tested happy and sad film clips while time pressure was manipulated by assigning 18 vs. 30 emergency events to the team over a 10-minute session. The pace of the high-stress condition ensured that 
participants would be alerted of a new emergency event before they had finished addressing the preceding one.

NeoCITIES is a scaled world simulation designed to mimic the situation assessment and resource allocation tasks of emergency crisis management teams. Teams of six were divided into three pairs (Police, Fire/EMS, and Hazardous Materials) to collectively address a series of emergency events requiring a range of responses and interactions within the team. Participants played the game at individual computer terminals isolated by dividers and had to coordinate all action and communication exclusively through the NeoCITIES client software using a text-based chat tool. The members of each pair had unique roles. One was the Information Manager (IM) who received incoming information about the emergency events. The other was the Resource Manager (RM) who had control over the resources to respond to the event, as well as access to reports on the success or failure of those resources. As time passed, an event that was neglected or incorrectly addressed escalated in severity, which increased the number of resources required to bring that event under control. Furthermore, task scenarios could include complex underlying plots (e.g. an emerging terrorist attack) hidden among other disconnected events (e.g. trash can fire, domestic dispute, car accident, etc.). For complete details of the construction and game-play of NeoCITIES, see McNeese et al. (2005).

Prior to the beginning of experimental trials, all participants received role-specific training on how to operate the simulation and effectively perform their roles. Only the chat between IM-RM dyads was analyzed here.

\subsection{Measurements}

Manual and automated text analysis methods were used to search for specific emotional cues. Verbal emotional cues were analyzed using the Linguistic Inquiry and Word Count software (LIWC; Tausczik \& Pennebaker, 2010), which is used to analyze 
text for attentional focus, emotions, social relationships, cognitive styles, and individual differences. This software provided seven measures: self-references (I, me, my), social words (indicating relationships or interactions), articles (a, an, the), big words (more than six characters), cognitive or thinking words (causal and insight words), positive emotions, and negative emotions. Nonverbal cues (vocal spelling, lexical surrogates, spatial arrays, grammatical markers, and minus features) were counted manually by the researchers.

According to the lens model, the emotion communication is influenced by psychological state and personality traits of the sender and receiver. The original study from which this chat data was drawn performed a range of personality trait assessments on all participants. These included the "Big Five" personality factors of openness to experience, extraversion, conscientiousness, agreeableness, and neuroticism, which were measured by NEO PI-R TM Five-Factor Personality Inventory (Goldberg et al., 2001). Other trait measures included the State Trait Anxiety Inventory (STAI; Spielberger, 1983) and locus of control (MMCS; Lefcourt, 1981). These measures provided the opportunity to incorporate individual differences in the current analysis, though the choice of traits analyzed here was strictly opportunistic given the data available for the present study, rather than motivated by specific hypotheses.

The stress manipulation was checked using the Short Stress State Questionnaire (SSSQ, Helton \& Garland, 2006). The mood manipulation was checked using items from the Positive and Negative Affectivity Scales (PANAS; Watson, Clark, \& Tellegen, 1988) and Short-Form State Trait Anxiety Inventory (Marteau \& Bekker, 1992).

\subsection{Results}

\subsubsection{Manipulation check}

Both mood and stress manipulations were successful. The measures of selfreported stress and mood states were averaged across both members of each dyad and analyzed using within-subjects ANOVAs (due to the small sample, the level of 
significance was relaxed to $\alpha=.10$ ). Participants reported higher positive affect in the happy condition $(M=1.80, S E=.12)$ than in the sad condition $(M=1.14, S E=.12)$, $F(1,56.98)=45.53, p<.01$, and likewise reported higher negative affect in the sad condition $(M=.81, S E=.07)$ than in the happy condition $(M=.19, S E=.07), F(1,57.14)$ $=91.30, p<.01$. The differences between the post- and pre-task measurements of the three dimensions of the Short Stress State Questionnaire (SSSQ) tested the time pressure stress manipulation. Stressed participants reported lower engagement $(M=.83$, $S E=.13)$ than non-stressed $(M=1.10, S E=.13), F(1,58.77)=3.31, p<.10$. Stressed participants reported more worry $(M=.19, S E=.08)$ than non-stressed participants $(M=$ $-.03, S E=.08), F(1,58.92)=4.20, p<.05$. No significant effect was found for the distress dimension.

\subsubsection{Emotional message cues}

Table 1 shows the results of factorial repeated-measures ANOVAs for each of the verbal and nonverbal cue categories. Only one main effect was found for mood while four were found for stress. There were no significant interactions between mood and stress. Mean counts of cues for the significant effects are shown in Table 2. Table 3 highlights the significant correlations between the personality trait measures and the verbal and nonverbal cues. No significant correlations were found for agreeableness, conscientiousness, neuroticism, or trait anxiety. 
Table 1. Factorial repeated-measures ANOVAs of verbal and nonverbal cues.

\begin{tabular}{lccccc}
\hline & \multicolumn{2}{c}{ Mood } & & \multicolumn{2}{c}{ Stress } \\
\cline { 2 - 3 } \cline { 5 - 6 } Measure & $F(1,41)$ & $r$ & & $F(1,41)$ & $r$ \\
\hline Vocal Spelling & 3.65 & & & $0.34^{*}$ & .63 \\
Lexical Surrogates & 3.51 & & & 1.84 & \\
Spatial Arrays & 0.20 & & & 1.06 & \\
Grammatical Markers & 1.12 & & & 0.38 & \\
Minus Features & 0.00 & & & $0.65^{*}$ & .65 \\
Self References & 0.75 & & & 1.43 & \\
Social Words & 1.07 & & & $4.31^{*}$ & .62 \\
Positive Emotions & 0.85 & & & $8.21^{* *}$ & .78 \\
Negative Emotions & 0.24 & & & 2.15 & \\
Cognitive Words & 0.89 & & & 1.78 & \\
Articles & 0.67 & & & & \\
Big Words & $9.18^{* *}$ & .82 & &
\end{tabular}

Notes: Effect sizes are only reported for significant results.

${ }^{*} p<.05,{ }^{* *} p<.01$.

Table 2. Effects of mood and stress on chat cue counts.

\begin{tabular}{|c|c|c|c|c|}
\hline \multirow[b]{2}{*}{ Measure } & \multicolumn{2}{|c|}{ Sad Mood } & \multicolumn{2}{|c|}{ Happy Mood } \\
\hline & $M$ & $S E$ & $M$ & $S E$ \\
\hline \multirow[t]{3}{*}{ Big Words } & 9.43 & 1.03 & 5.84 & 0.85 \\
\hline & \multicolumn{2}{|c|}{ No Stress } & \multicolumn{2}{|c|}{ High Stress } \\
\hline & $M$ & $S E$ & $\bar{M}$ & $S E$ \\
\hline Vocal Spelling & 0.82 & 0.26 & 0.27 & 0.07 \\
\hline Self References & 4.20 & 0.66 & 6.05 & 0.66 \\
\hline Negative Emotions & 1.05 & 0.27 & 2.41 & 0.54 \\
\hline Cognitive Words & 4.06 & 0.63 & 6.48 & 0.69 \\
\hline
\end{tabular}

Table 3. Non-parametric correlations $\left(r_{s}\right)$ for trait measures and chat cues.

\begin{tabular}{lccc}
\hline Measure & Extraversion & $\begin{array}{c}\text { Openness to } \\
\text { Experience }\end{array}$ & $\begin{array}{c}\text { Locus of } \\
\text { Control }\end{array}$ \\
\hline Vocal Spelling &. $\mathbf{2 4}^{* *}$ & .03 & $-.19^{*}$ \\
Lexical Surrogates &. $\mathbf{2 0}^{* *}$ & .14 & -.15 \\
Spatial Arrays & -.02 & .01 & .15 \\
Grammatical Markers & .07 & .15 & -.09 \\
Minus Features & $.25^{* *}$ & $.18^{*}$ & -.14 \\
Self-References & .12 & .08 & -.11 \\
Social Words &. $\mathbf{2 4}^{* *}$ & $.17^{*}$ & .01 \\
Positive Emotions & $.18^{*}$ & $.16^{*}$ & .06 \\
Negative Emotions & .10 & .10 & -.03 \\
Cognitive Words & .07 & $.21^{* *}$ & $-.16^{*}$ \\
Articles & $.16^{*}$ & .01 & .01 \\
Big Words & .11 & .10 & -.06 \\
\hline${ }^{*} p<.05,{ }^{* *} p<.01$. & &
\end{tabular}




\subsection{Discussion}

A significant change in the use of big words was the only significant effect for mood, with sad participants using nearly twice as many as happy participants. This was somewhat surprising, as we expected to see some difference in at least the negative emotion category, given the increase of negative terms used by participants in a negative mood in Hancock et al. (2008). A possible explanation for the increase in big words may lie in the effect of a negative mood to focus attention on local details rather than the big picture (Gasper, 2003), as sad participants may dwell longer on technical specifics than happy participants. Sexton \& Helmreich (2000), analyzing cockpit communication, found that flight engineers used more big words than captains and first officers, possibly because the information they communicate is necessarily more technical than the others. However, big words negatively correlated with performance in the cockpit overall, possibly due to either the lack of a concise and succinct vocabulary, or a tradeoff between the cognitive effort to speak elaborately and the ability to maintain effective situation awareness.

Participants under stress produced significantly more self-references, negative emotions, and cognitive words, but fewer vocal spellings than non-stressed participants. Pfaff (2012) showed that participants under time pressure stress engaged in greater amounts of sense-making behavior, trying to figure out what someone was doing or what happened on a certain event, which would account for the increase in self-references and cognitive words. The cognitive words category includes references to causation and knowledge of relationships (e.g. cause, effect, know, maybe, should, would). Messages under these conditions are frequently explaining or justifying actions taken, which would require such word choices (e.g. "I think another fire truck should take care of it"). An increase in words suggesting negative emotions is also to be expected during task overload conditions, which were designed to frustrate the player through frequent task 
switching. Most interesting is the significant decrease in vocal spellings, which may reveal that using such a technique requires additional time and cognitive effort to consider and execute, indicating that one known mechanism for coping with the restricted emotional bandwidth of text-based IM appears to be of limited use under time pressure.

With respect to the Big Five personality factors, there was a significant correlation between extraversion and several of the chat cue categories. While extraversion could be readily linked to talkativeness in general, there was no significant correlation between extraversion and the overall amount of chat. Therefore, there is something about these particular cues (vocal spelling, lexical surrogates, minus features, social words, positive emotions, and articles) that extraverts appear to employ more than introverts. The use of vocal spelling and lexical surrogates are especially intriguing as paralinguistic cues that mimic real speech. As such, this finding corresponds to the findings of Scherer (1978), which was able to detect extraversion directly from an assessment of voice quality, specifically vocal effort and dynamic range.

Openness to experience was the only other "Big Five" personality trait with significant correlations to any chat cues (minus features, social words, positive emotions, and cognitive words). It shares three of these four correlations with extraversion, in part due to a significant correlation between extraversion and openness in this sample $\left(r_{\mathrm{s}}\right.$ $=.28, p<.01)$. Individuals high on the openness scale tend to have richer vocabularies, vivid imaginations, and be more sensitive to emotion and creative ideas. Individuals low on the openness scale tend to favor conventional approaches, which could explain the correlation with minus features. Such individuals may be more likely to adhere to conventions of punctuation and formatting in text, while those high on the openness scale may simply be less diligent about their typography in IM. 
Locus of control was inversely correlated with vocal spellings and cognitive words, meaning those with a high internal locus of control were more likely to produce these cues (a high score on the MMCS indicates an external locus of control). In organizational settings, internals tend to seek out and process complex information more than externals (Spector, 1982), which aligns with the correlation with cognitive words. Apart from defining the category, Boonthanom (2004) does not provide any theoretical justification for why an individual would utilize vocal spellings in text-based CMC, though its use did diminish under time pressure. Internals tend to be more motivated to perform, and may possibly exert the effort to enrich their textual expression despite the time pressure. The current data is unfortunately insufficient to show whether this moderating effect exists.

\subsection{Conclusion}

This study served to extend previous studies on emotional communication in CMC (e.g. Boonthanom, 2004; Hancock et al., 2007; Hancock et al., 2008) and the results confirmed that studying text-based emotional cues in IM merits further study. Although this study presented an initial effort to understand emotion expression in textbased CMC, following contributions can be explained.

The results of this study provided empirical support for Brunswik's lens model (1956) in synchronous text-based CMC, demonstrating that situational context and personal traits of encoder can affect emotional communication. These results also contributed toward sentiment analysis and automatic extraction of opinions and emotions from text. Detection of emotional cues applied in text-based CMC can inform different models which are employed in text analysis (Alm, Roth, \& Sproat, 2005; Pang, \& Lee, 2008). Armed with these exploratory findings, the results of this study provided the following helpful insights for future studies and specifically subsequent studies of this dissertation. 
Future studies need to cultivate controlled emotionally-laden situations in which participants are more likely to apply text-based emotional message cues to express their emotions, rather than opportunistically mine data sets such as these, which are more task-focused than expressly intended to elicit emotional exchanges between partners. Future studies also need to examine chat data from a more diverse participant pool (in terms of age, gender, and ethnicity) than this overwhelmingly young, Caucasian male sample. One of the most used and researched forms of non-text-based input methods in IM is the use of graphic emoticons. Future studies need to operationalize emoticons in addition to strictly text-based emotional message cues.

This study provided empirical support for Brunswik's lens model (Brunswik, 1956) in text-based CMC, which argues that situational context and personal traits of the encoder can affect emotional communication. Future studies also need to apply the modified version of the lens model to characterize the influences of other emotional states (relaxed, angry, happy, sad), on the proportions of different emotion-related cues used during informal IM conversations in controlled emotionally-laden situations.

The above insights were applied to design study III that explored how people actually communicate their emotion in instant messaging. However, before moving to that study, since the main goal of this dissertation was to conduct a design research focusing on multi-touch gesture based design solutions, an exploratory design research was conducted to investigate the potential of multi-touch gestures to support emotional communication in this medium. The following section will explain the design research. 
Chapter 4. Study II: Designing Multi-Touch Gestures to Support Emotional Expression in IM

\subsection{Introduction}

The simplicity and variety of multi-touch gestures provide unique opportunities for new forms of human-computer interaction. However, using such gestures to support emotional communication in IM has received limited attention. While Study I mined communication data to explore how people express their emotion in IM, the main goal of this exploratory study is to, through design research, explore the potential of multi-touch gestures in instant messaging and develop a set of multi-touch gestures to support emotional expression in this medium.

\subsection{Research}

The main goal of this stage was to, through a qualitative research study, examine how IM users express their emotions in IM. The "5-Why" method (Ogura, 2002) was used to explore the main challenges users encounter in emotional expression in IM and the "role-playing" method to identify text-based cues users apply to overcome those challenges.

The "5-Why" method was conducted with five IUPUI students, four female and one male (24-29 years old, $M=27.6)$ to identify the main challenges they encounter in expressing their emotion via IM. "5-Why" is composed of an iterative process to uncover unclear causes and effects of a problem (Ogura, 2002). It starts from a first why question for a specific statement and the participant providing an answer. Participants then propose another why question based on that answer and then repeat this process for a total of five questions. " 5 -why" method was selected for this stage of the study since we were interested in exploring challenges users encounter in expressing their emotion via IM to identify the main focus of our design. We started with the question of "Why is emotion communication via IM sometimes challenging?" Participants were asked to write 
their answers on sticky-notes. We then proposed further questions based on their answers. Some of the questions that were proposed were: "Why are words not enough to express your emotion?" "Why is it difficult to read the emotion of the person you are chatting with?" "Why do you have to use IM?" "Why is it challenging not being able to communicate facial expression and voice in text?"

An affinity diagram (Figure 3) was created, which showed conveying facial expression, expressing the intensity of their emotion, and providing feedback as three main challenges users encounter in emotional expression in IM. Some of the answers in the process of " 5 -why" method were: "limited number of emoticons are not enough to express all facial expressions", "since there is no voice in IM, sometimes it's hard to show how angry or happy you are in text", and "you can not figure out the other person's feeling." Analysis also revealed some advantages of IM over face-to-face communication such as communication speed, the ability to send short messages, and multi-tasking.

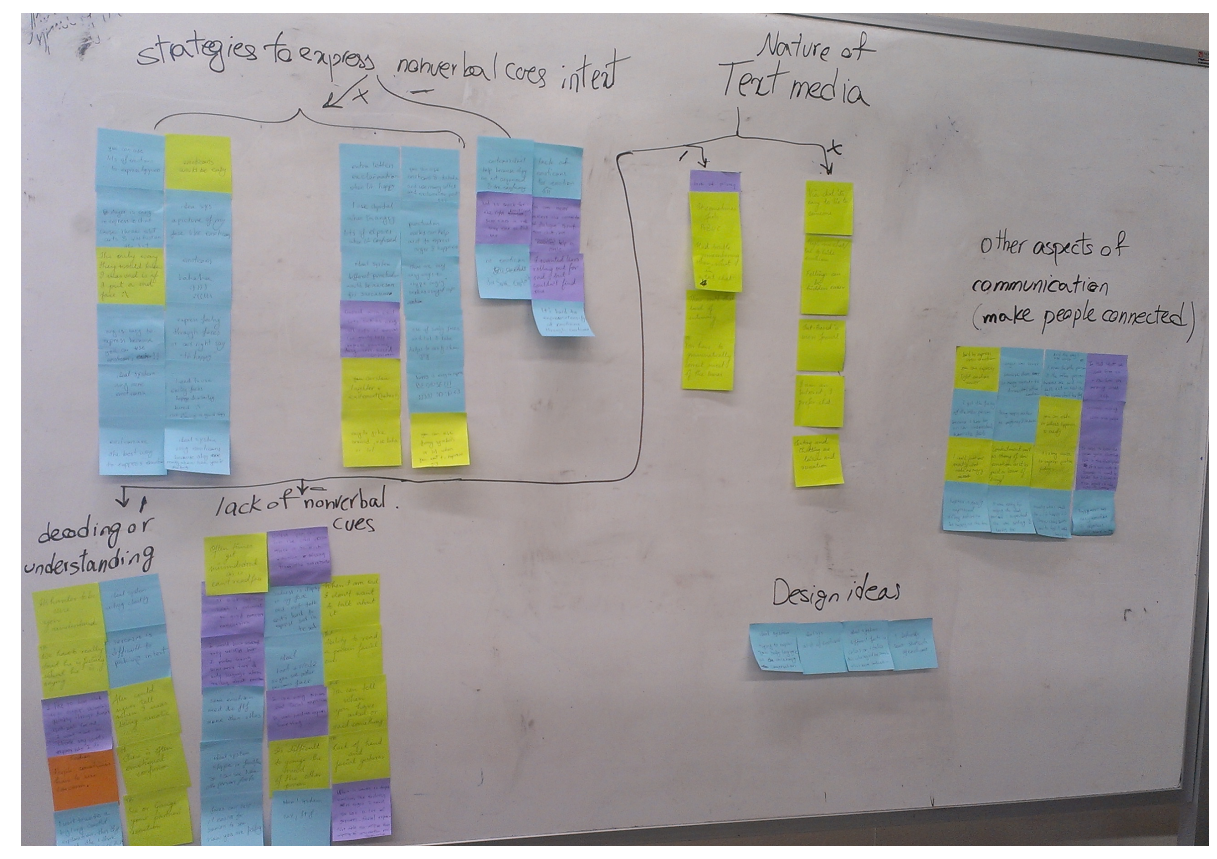

Figure 3. Affinity diagram 
Based on the results of the " 5 -Why" method, the main focus of our design aimed to support facial expression, express the intensity of emotion, and provide feedback in IM communication. We then used a "role-playing" method to explore different text-based cues participants use to overcome these three main challenges identified from the "5why" method.

In the "role-playing" phase, six topics were developed to involve participants in emotional conversations so that they were more likely to express their emotions in different levels of intensity and give feedback to their partners. Topics were developed to elicit happiness in three levels of intensity (winning a lottery, getting a free lunch, and passing the course with good grade), sadness in two levels of intensity (your pet just died and your cousin passed away today), and anger in one level of intensity (your boyfriend/girlfriend is cheating on you). Eight IUPUI students in four pairs, two male and six female $(22-28$ years old, $M=23.8)$ participated in this phase of the study. Two partners of each pair received email on the instruction of the study with the list of six topics. They were asked to role-play in a chat conversation, from separate locations, via Google chat. They were also asked to chat for five minutes for each topic and email all the chat conversations to the researchers.

Data was gathered and emotional cues participants used were categorized into three categories of facial expression (e.g. :( : : :-D : :))) :-O :-P), intensity of emotion (vocal spelling such as sooo and weeell, upper case such as SO MAD) and feedback (e.g. Haha, lol, umm, OMG, really?).

\subsection{Design}

In this stage multi-touch gestures were created equivalent for all the text-based emotional cues that were identified in the previous stage. Brainstorming was done for this stage of the project by the researcher and three other $\mathrm{HCl}$ students. Random objects (e.g. ball, small cube, pillow) and sign language gestures were used to stimulate 
idea generation. Researchers generated as many gestures as they could in five minutes for each emotional cue. The output was 3-10 gestures for each emotional cue. Design ideas were then evaluated and narrowed down to one or two gestures for each emotional cue based on the IM advantage criteria participants reported in the "5-Why" method (communication speed, the ability to send short messages, multi-tasking). The result was a set of multi-touch gestures equivalent to emotional cues users used to express their emotions in three categories of facial expression, intensity of the emotion (Figure 4) and feedback (Figure 5).
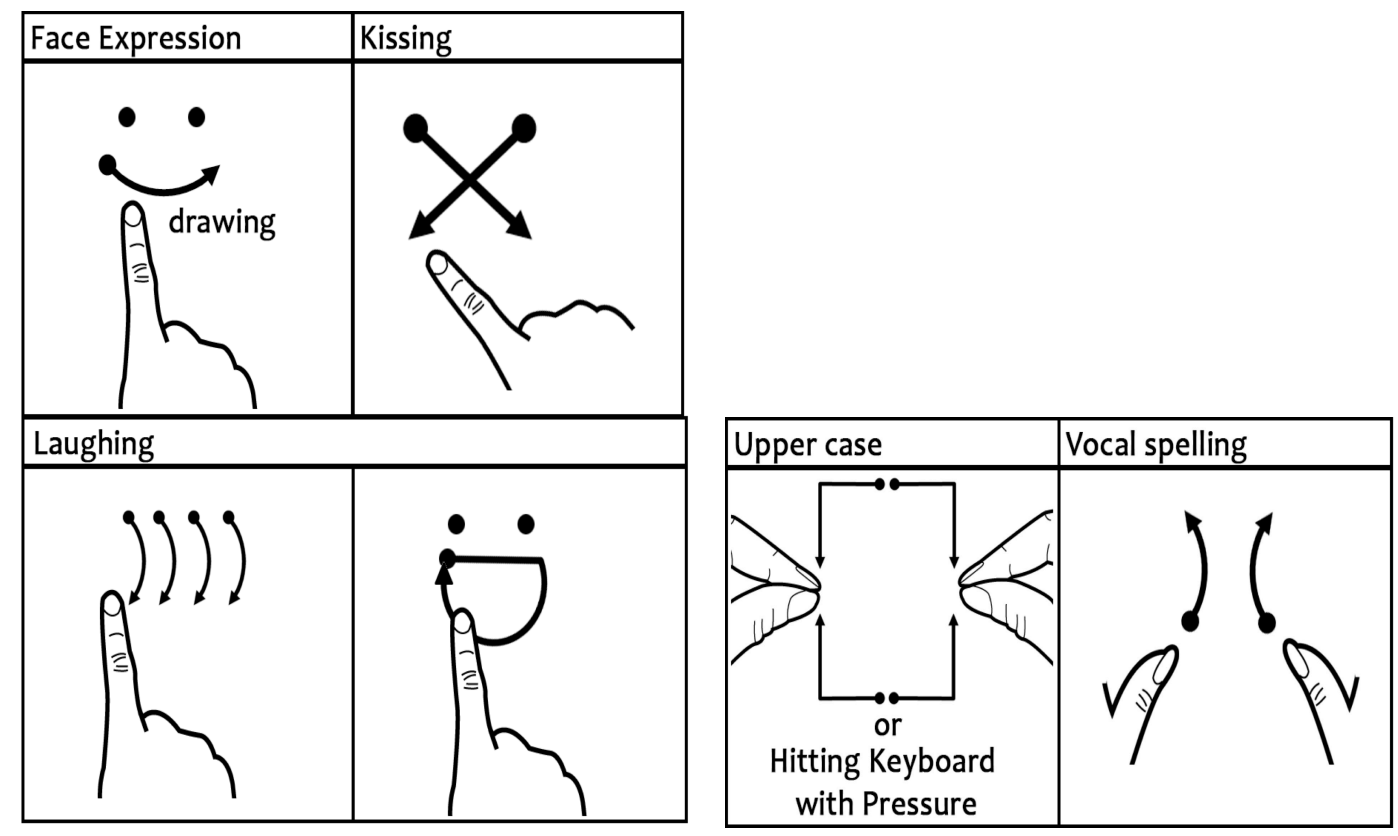

Figure 4. Facial expression and intensity gestures 


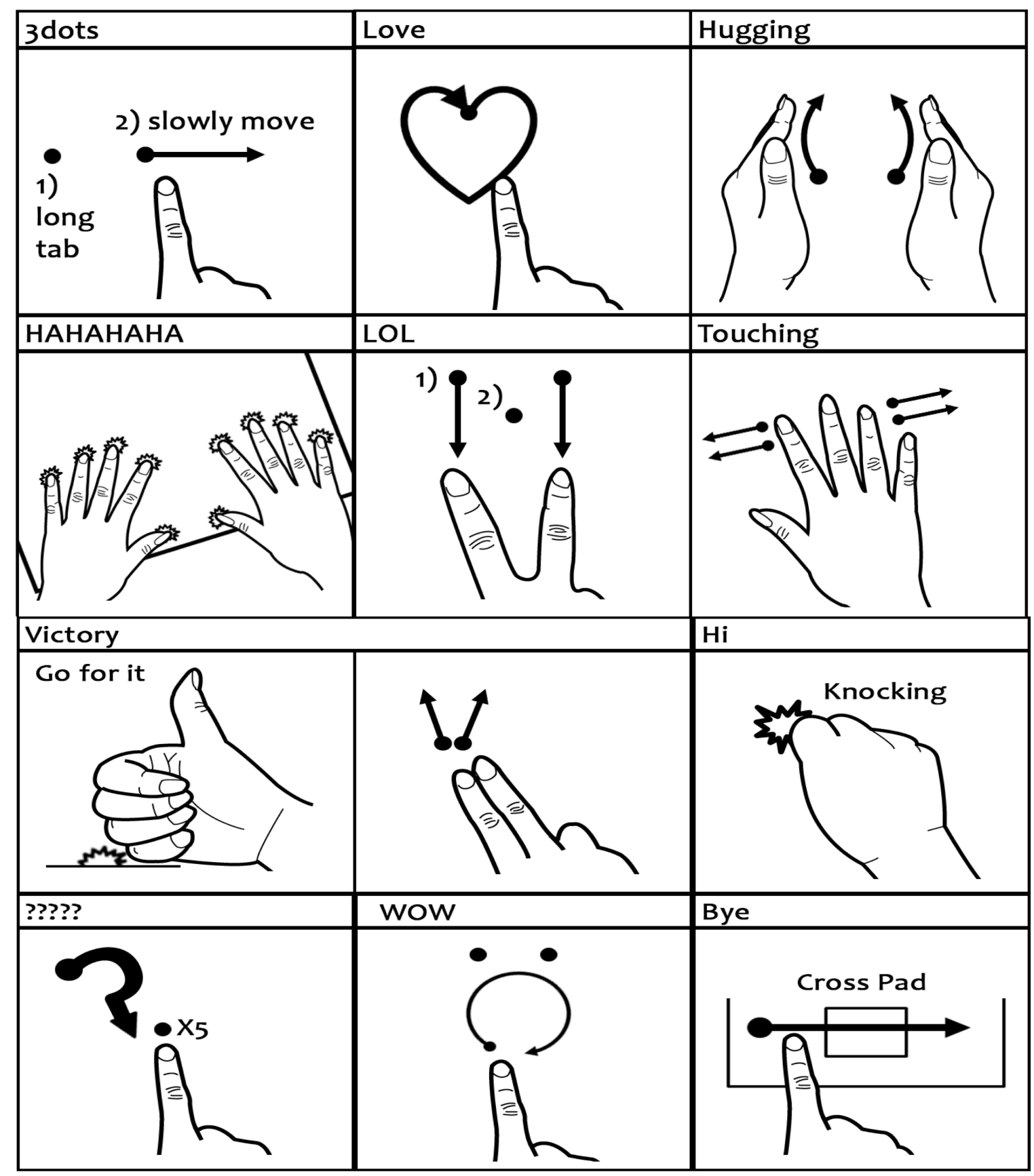

Figure 5. feedback gestures 


\subsection{User Evaluation}

An exploratory usability evaluation was conducted by six IUPUI students, two male and four female (range $23-29$ years, $M=24.6$ ). Three scenarios (sad, happy, and angry) wee created and highlighted emotional cues that could be expressed by gestures in those scenarios. Participants were asked to go through and type the chat scenarios they were given. As they got to the highlighted emotional cue, they needed to create an equivalent gesture for that specific cue. They were asked to manually express their gestures on two blank yellow sticky notes that were placed on each side of a laptop touch pad (Figure 6). They were then introduced to the gesture that design researchers had created for that specific emotional cue and asked which one they preferred, the designers gesture or the gesture they created, and why. The same process was used for all highlighted emotional cues in all three scenarios. Users were asked to think aloud when they were creating their own gestures or using designers' gestures. All sessions were video recorded for later analysis.

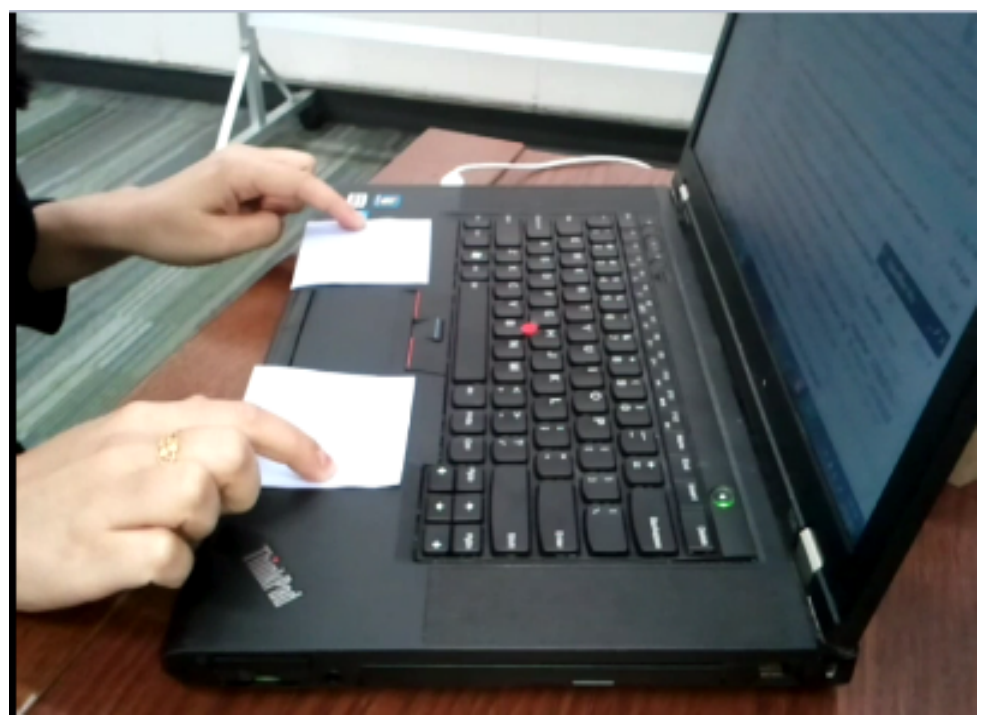

Figure 6. User evaluation setting. 
Overall participants provided positive comments on using gestures to express their emotions in IM. Feedback gestures received the most positive comments from users since they were easy to use and they could use gestures instead of typing a word or phrase such as "really?", "go for it", or "hahaha." Facial expression gestures received positive comments when they were used as feedback. However, participants reported this group of gestures was not effective when they were used in the middle of a sentence. They reduced their typing speed and participants preferred to type those facial expressions instead of using gestures. Finally, gestures with the purpose of supporting intensity were the least favorite from the users' perspective. However, vocal spelling gestures, in which participants used the keyboard and touch-pad simultaneously, received more positive feedback than the upper case gesture, in which participants needed to switch between keyboard and touch-pad.

\subsection{Discussion}

Although our study should be seen as an initial effort to examine the potential of multi-touch gestures to support expression of emotion in IM, the approach we applied in this study presented our design research process and provided a framework for future design studies in this area. The results of the user evaluation showed how participants perceived and reacted to multi-touch gestures for emotional expression in IM. Participants were not interested in gestures that needed them to keep switching from keyboard to touch-pad and vice versa (e.g. smiley faces in the middle of a sentence, or upper case), as it reduced their typing speed, which was reported as one of the advantages of IM communication. Designers need to consider the nature of IM communication (i.e. short and fast messages) in their design in addition to supporting emotional communication, which is challenging.

Gestures with the purpose of supporting intensity received the least positive feedback from participants. One possible explanation of this result could be the relation 
of this category of the emotional cues to vocal cues, which is difficult for users to relate to gestures. Alternately, it may simply be more efficient to use the keyboard for these cues, rather than invent new gestures. Therefore supporting voice cues through gestures may be more challenging compared to other nonverbal behaviors and future studies need to be open to different input options.

\subsection{Conclusion}

As an exploratory study, there were several limitations that pointed toward improvements for future studies and specifically the subsequent study III (chapter 5) and design process (chapter 6 ) of this dissertation.

One of the limitations of this study was the small sample size and the higher number of female compared to male participants. Since it was a preliminary study, convenience sampling was used. However, future studies need to use a bigger sample size and higher number of males for upfront research and design process.

Another limitation of this study was the emotion elicitation strategy. Participants were asked to act out the different emotions of happy, sad, and angry. The role-playing nature of their emotional expression might have affected their emotional communication and made it unnatural or exaggerated. Future studies need to explore emotional cues by cultivating controlled emotionally-laden situations, in which participants are more likely to engage in natural emotional conversation.

This study focused on gestures as input technology from the sender's perspective, while the output will remain the same as emoticons and other emotional cues senders apply in IM. Future studies need to focus on alternative output technologies for visualization of gestures from the receiver's perspective.

This study examined emotional expression in different emotions of sadness, happiness, and anger in each role-playing phase. However, the emotional cues identified from all conversations with different emotional states were combined, and the 
design ideation phase was done for all the emotional cues. Future studies need to focus on the specific emotional state from the research phase to design ideation and evaluation.

The findings of the " 5 -why" method revealed three main challenges users think they encounter in IM communication. What challenges users encounter in reality however, may be different than what they think. A more in-depth qualitative research in future studies may reveal other challenges users encounter in IM.

Text-based emotional cues in this study were manually identified from participants' conversations. However, future studies need to conduct a more in-depth conversation analysis to study the structure and the sequential organization of the conversations and how emotions are expressed, responded to, and worked through in IM conversations. Conversation analysis may also reveal a wider range of strategies and emotional cues participants apply to communicate emotions in the context of IM conversations.

The results of the user evaluation showed how participants perceived and reacted to multi-touch gestures for emotional expression in IM. Participants were not interested in gestures that needed them to keep switching from keyboard to touch-pad and vise versa (e.g. smiley faces in the middle of a sentence, upper case), as it reduced their typing speed, which was reported as one of the advantages of IM communication. Designers need to consider the nature of IM communication (i.e. short and fast messages) in their design in addition to supporting emotional communication, which is challenging.

Based on the insights that were gained from studies I and II, and continuing the trajectory of the prior research in the area of emotional communication in instant messaging, study III was designed and conducted. This will be explained in the following chapter. 
Chapter 5. Study III: How do you IM when you get emotional?

\subsection{Introduction}

The main goal of this study is to investigate how people actually communicate their emotions in IM through a mixed-methodology approach. This study was designed based on the main insights from studies I and II, and sought to address some limitations of the previous studies in this area and extend their findings in several aspects.

The first aspect is the emotion elicitation strategy. In previous studies (Hancock, Landrigan, and Silver, 2007; Walther, Loh, Granka, 2005), participants were asked to act likable or unlikable, and happy or sad. As Hancock et al. (2008) explained, the roleplaying nature of their emotional expression in prior studies might have affected their emotional communication and made it unnatural or exaggerated. In the study reported here, short video clips were used to induce four specific moods, followed by open-ended text-based chat accompanied by memory elicitation designed to maintain those moods and encourage participants to express their emotions more naturally during chat. Therefore, text-based IM communication was explored by cultivating controlled

emotionally-laden situations in which participants were more likely to engage in natural emotional conversation and apply text-based emotional cues to express those emotions.

The second innovative aspect of this study is the wider range of moods studied to investigate the emotional cues in text-based IM. Compared to previous studies (Hancock, Ciaccio, and Lin, 2008; Hancock, Landrigan, and Silver, 2007; Walther, Loh, Granka, 2005), which examined a limited range of positive and negative emotions (generally happy or sad), this study investigated a range of four distinct emotions of relaxed, sad, happy, and angry. These are four major emotional states that can be expected to occur frequently in daily life. In Russell's (1980) circumplex model of affect, these four different emotions occupy four separate quadrants in a two-dimensional space composed of pleasure-displeasure and degree of activation. 
The third innovative aspect of this study is the large set of text-based emotional cues selected for detection and analysis. A relatively new and novel set of verbal and nonverbal cues was extracted from Boonthanom's (2004) study of asynchronous CMC (email). This set includes emotion words (e.g. happy, angry), vocal spelling (altering spelling to mimic a specific vocal inflection, e.g. weeeell or soooo), lexical surrogates (textual representations of vocal sounds that are not words, e.g. uh huh, haha), spatial arrays or emoticons (pictographs constructed from punctuation and letters, e.g. :-( for a sad face, or :-D to indicate laughing), manipulation of grammatical markers (alterations of the presentation of words, e.g. all capital letters, strings of periods or commas), and minus features (deliberate or inadvertent neglect of conventional formatting elements, e.g. abbreviation and acronyms, lack of capitalization or paragraphing). Additional cues were derived from the existing literature on the text-based CMC. Hancock et al. (2007) showed that degree of agreements, verbosity, and punctuation are three strategies participants use to express positive versus negative emotion in text-based CMC. Therefore, the following categories were also explored in this study: assent (e.g. agree, OK, yes), negation (e.g. no, not, never), punctuation, and number of words per conversations as an indicator of verbosity. Note that, based on Boonthanom's (2004) cue taxonomy, vocal spelling, lexical surrogates, spatial arrays/emoticons, punctuation, minus features, and manipulation of grammatical markers are called nonverbal emotional cues since they mimic visual and aural nonverbal behaviors in face-to-face communication, such as facial expression, tone of voice, body gesture, or posture (Boonthanom, 2004; Hancock, 2004). We categorized positive, negative, and swear words as verbal emotional cues.

The fourth aspect of this study was the combination of quantitative and qualitative analysis. The main focus of this study is to, through a mixed methodology, quantitatively and qualitatively explore emotional communication in instant messaging. 
The quantitative content analysis was conducted by counting the frequency of textbased emotional cues and focused on how four emotional states (relaxed, angry, happy, sad) influenced the type and quantity of emotion-related cues used during informal conversations between college friends in IM. While qualitative content analysis approach looked closely at how emotions were expressed, responded to, and worked through in IM conversations. Qualitative analysis in addition to quantitative generated an in-depth, holistic understanding of communication of emotion in instant messaging. The results of this study provided a valuable foundation for the design process. The following sections explain the experiment methodology and the results of the quantitative and qualitative analysis.

\subsection{Methods}

\subsubsection{Participants}

Twenty college students in ten pairs of friends, three males and 17 females, ranging from 18 to 31 years old, received a $\$ 10$ gift card for their participation in this study. We required partners to be friends who knew each other for at least six months so they were more likely to IM each other in real life, to express their emotions, and engage in natural emotional conversation (Wagner and Smith, 1991; Parkinson, Fischer, and Manstead, 2005; Derks, Fischer, and Bos, 2008; Ramirez and Broneck, 2009). Despite the motivation that was gained through previous studies to recruit an equal number of male and female participants, this study could not overcome that limitation because of the time limitation and convenient sampling.

\subsubsection{Procedure}

Each pair of friends arrived at the laboratory together, and each friend was randomly assigned to separate rooms equipped with similar equipment (computer, table, and chair). After sitting at the computers, they were asked to sign a consent form and answer several demographic questions (See appendix A). 
Participants were informed that the purpose of this study was to learn about textbased communication, with no mention of emotion. Telling participants the actual goal of the study might have increased the demand effects in which participants might not naturally achieve the desired emotional state and by either resisting or pretending to be in the mood.

The study used a within-subjects design. The experiment included two phases, repeated for each of the four emotional conditions of relaxed, angry, happy, and sad. The first phase was the mood induction. For each mood induction, a short video clip was selected to elicit the condition mood. Partners were asked to watch the video, followed by a manipulation check survey using the 28 emotion items from Russell (1980) (See appendix B). Among different procedures to elicit emotions or induce moods, such as imagination, images, film/story, sound/music, or social interaction, the meta-analysis results by Westermann et al. (1996) argued that the film/story mood induction procedure was the most effective procedure when subjects are treated individually (Westermann, Spies, Stahl, and Hesse, 1996). The relaxed clip was treated as the baseline condition and always shown first. To avoid carryover effects, suggested by Rottenberg, Ray, and Gross (2007), video clips of the same valence were shown in a blocked order. Therefore, the happy video clip was shown as the third video and anger and sad video clips were shown randomly in second and fourth position.

The second phase was informal chat via IM. The goal of this phase was to keep participants in the emotional state that was elicited using the video clip and engaging them in a chat conversation likely to include expression of their emotions. A memory elicitation technique (Morris, 1989) was used to reach the goal of this phase. Participants were asked to trigger each other's memory to remember different life experiences they had related to the emotion of the film they just watched, and talk about them for ten minutes via Google Chat before watching the next movie. The memory elicitation 
technique is favored by several researchers since they directly trigger individual experiences of emotion (Morris, 1989). Participants were given some sample questions (See appendix C)to use for memory elicitation (e.g. "Have you had any experiences similar to what you watched in the video?" "What makes you relaxed/happy/sad/angry?").

After watching and chatting about all four movies, participants answered a short survey (See appendix D) on how satisfied they were expressing their emotions in chat conversation and why. They were asked to report their satisfaction in a 7-point scale of 1 (totally dissatisfied) to 7 (totally satisfied). Other questions were also asked such as "Which emotion was the hardest/easiest to express through text-based chat? Why?" and "What differences do you see between expressing your emotion in IM compared to faceto-face?" At the end, participants were compensated and dismissed.

\subsubsection{Measurements}

For the manipulation check, participants reported their feelings after watching the video clip on a 5-point scale of 1 (slightly or not at all) to 5 (extremely), for each of the 28 emotion terms in Russell's circumplex model (1980). Clusters of emotions were created from the four highest-rated emotions in each condition. This created four roughly orthogonal emotion clusters, one in each quadrant of the two-dimensional circumplex model, in which the horizontal axis represents emotional valence (unpleasant on the left and pleasant on the right) and degree of activation on the vertical axis (Figure 7). The relaxed mood was calculated by taking mean of four emotions of at ease, serene, calm, and relaxed. The same process was done for measuring happy (happy, glad, pleased, delighted), angry (annoyed, frustrated, angry, tense), and sad (gloomy, sad, depressed, and miserable) moods. All four moods of relaxed, anger, happy, and sad were calculated for each of the four conditions. 


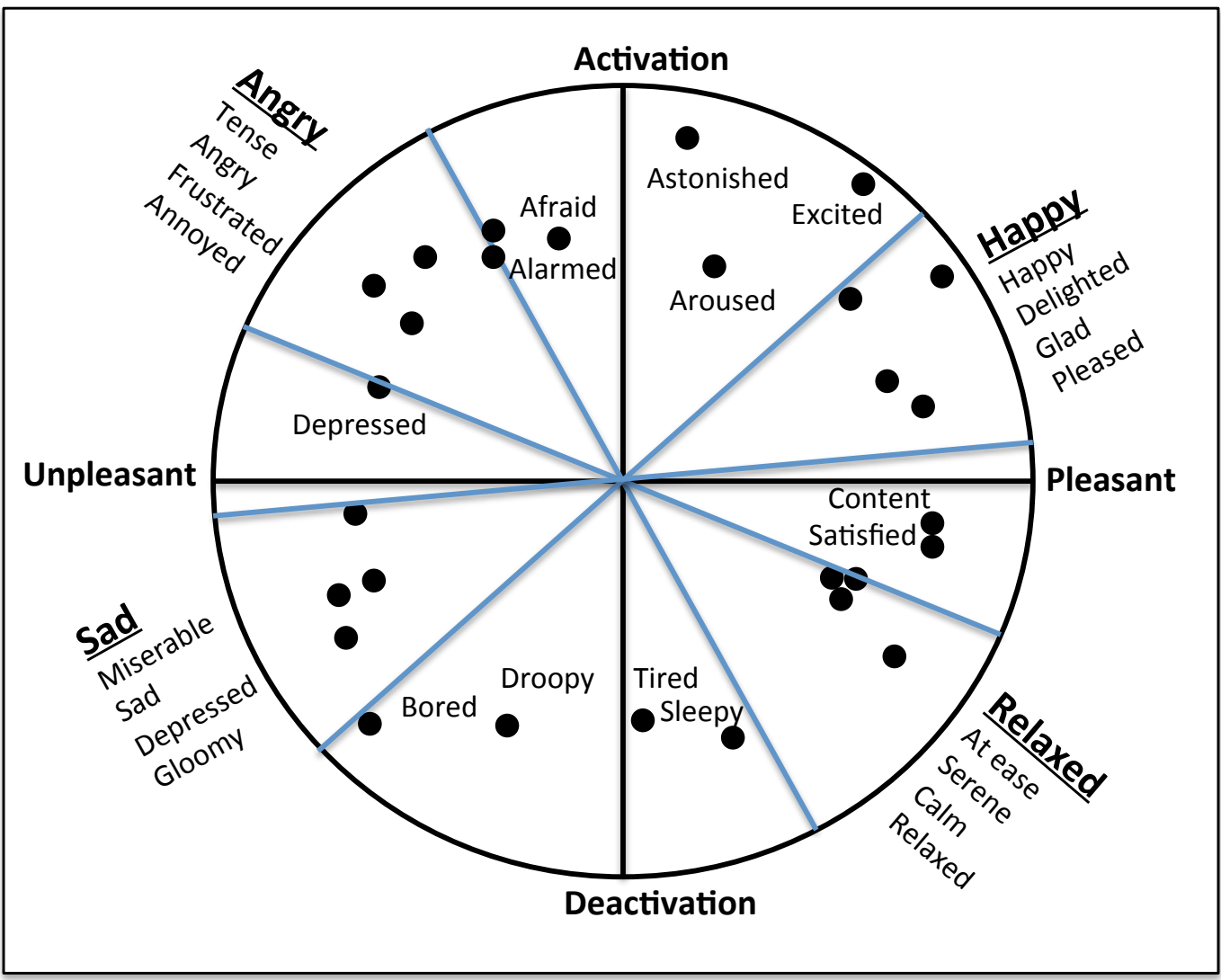

Figure 7. Emotion clusters selected from Russell's (1980) circumplex model, showing valence as the horizontal axis, and activation as the vertical axis.

Verbal emotional cues including positive emotion words, negative emotion words (angry, sad, anxiety), and swear words were counted using the Linguistic Inquiry and Word Count (LIWC) software (Tausczik and Pennebaker; 2010). LIWC was also used to count the assent and negation words, big words (words > 6 letters), fillers (blah, I mean, you know), cognitive (e.g. know, think), and perceptual (e.g. see, hear, feel). All nonverbal cues except punctuation (vocal spelling, lexical surrogates, spatial arrays/emoticons, minus features, and grammatical markers) were counted manually by the researchers. Punctuation was counted using LIWC. Since verbal cues identified by LIWC represent percentages of total words produced in each condition, we calculated nonverbal cues as percentages of total words produced in each category. 


\subsection{Results (Quantitative analysis)}

The main goal of the quantitative analysis is to investigate the effect of users' emotional state on different emotional cues they apply in text-based IM.

\subsubsection{Manipulation Check}

The mood manipulation was checked first to test whether the levels of each emotion (relaxed, angry, happy, and sad) were significantly higher than the other three for each of the corresponding mood inductions. Since the distribution of moods in none of the conditions was normal, the manipulation check was done by conducting Friedman's ANOVAs for all four emotions in each condition. The results were significant in all conditions at $p<.001$ : relaxed $\left(x^{2}(3)=43.43\right)$, angry $\left(x^{2}(3)=45.65\right)$, happy $\left(x^{2}(3)\right.$ $=54.71)$, and $\operatorname{sad}\left(X^{2}(3)=52.14\right)$. Results of pairwise comparisons of the means using a Bonferroni correction are in Table 4.

Table 4. Mean emotion levels by condition (SD in parentheses)

\begin{tabular}{ccccc}
\hline \multirow{2}{*}{ Measure } & \multicolumn{5}{c}{ Condition } \\
\cline { 2 - 5 } & Relaxed & Angry & Happy & Sad \\
\hline Relaxed & $\mathbf{2 . 8 2 a}(\mathbf{0 . 9 3})$ & $0.14 \mathrm{a}(0.28)$ & $1.75 \mathrm{a}(0.83)$ & $0.11 \mathrm{ab}(0.25)$ \\
Angry & $0.17 \mathrm{~b}(0.28)$ & $\mathbf{1 . 8 3 b}(\mathbf{0 . 7 3})$ & $0.07 \mathrm{~b}(0.23)$ & $0.84 \mathrm{a}(0.76)$ \\
Happy & $1.76 \mathrm{a}(0.99)$ & $0.00 \mathrm{a}(0.00)$ & $\mathbf{3 . 1 8 a}(\mathbf{0 . 6 9 )}$ & $0.00 \mathrm{~b}(0.00)$ \\
Sad & $0.14 \mathrm{~b}(0.20)$ & $1.01 \mathrm{~b}(0.77)$ & $0.01 \mathrm{~b}(0.06)$ & $\mathbf{2 . 5 6 c}(\mathbf{0 . 9 2})$ \\
\hline
\end{tabular}

Means in columns not sharing a letter are significantly different at a Bonferroniadjusted $\alpha=.008$.

The results of the pairwise comparisons for each mood among four conditions showed that the desired emotional state was highest compared to the other three (noted in bold), though more in terms of valence than activation. Participants reported the negative emotion sad significantly higher than the other three emotions in the sad condition. However, in the remaining conditions, the two positive emotions were significantly different from the two negative emotions in their respective conditions, but the two same-valenced emotions were not significantly different from each other. This suggests that the manipulation was highly successful in terms of emotional valence 
(positive or negative), but only partially successful in terms of activation, as only the sad condition produced an emotional response significantly less activated than the angry emotion. Alternately, the emotion clusters simply may not have included items high enough in activation to produce a significant difference. This result may also be due to an emotional ceiling effect. Participants generally arrive in the laboratory in a positive mood, causing negative mood manipulations to have a much stronger effect than positive mood manipulations. However, even without a neat and mutually exclusive division of the four emotional responses, the four mood conditions successfully produced four clearly distinguishable and appropriate mood profiles, which was the goal of the manipulation.

\subsubsection{Emotional Cues}

Table 5 shows the results of Friedman repeated-measures ANOVAs for each of the cues categories. There were significant differences among all categories, except big words (words > 6 letters), filler (I mean, you know), emoticons, anxiety, cognitive, perceptual, and negation words in the four conditions. Follow-up pairwise comparisons were conducted for each condition using a Bonferroni-corrected level of significance $(.008)$

\subsubsection{Survey}

Overall, participants were satisfied (average response: 5.9/7) with emotion expression to their friends via IM communication. The main reason of their satisfaction was reported as comfortable conversations they had with friends that have known them for a long time. For example, "It helps to know the person you're chatting with in the first place. If it was with a stranger it would be harder but I found it easy because of the way we communicate already and then we just transferred that to this chat room" "I feel very comfortable talking to (friend's name). It almost felt as though we were chatting at home." "I feel like (friend's name) knows me well enough to understand what tone I am using 
Table 5. Mean percentages of verbal and nonverbal cues by condition (SD in parentheses).

\begin{tabular}{|c|c|c|c|c|c|}
\hline \multirow[b]{2}{*}{ Cue Categories } & \multicolumn{4}{|c|}{ Condition } & \multirow[b]{2}{*}{$x^{2}(3)$} \\
\hline & Relaxed & Angry & Happy & Sad & \\
\hline \multicolumn{6}{|l|}{ Verbal } \\
\hline \multicolumn{6}{|l|}{ Emotional Cues } \\
\hline Affect words & $8.66 \mathrm{ab}(2.68)$ & $8.01 \mathrm{a}(2.93)$ & $11.22 b(3.02)$ & $8.78 \mathrm{ab}(2.27)$ & $9.60^{*}$ \\
\hline Positive words & $7.46 \mathrm{ab}(2.17)$ & $4.01 \mathrm{a}(1.77)$ & $9.49 b(2.71)$ & $3.98 a(1.91)$ & $27.42^{* * *}$ \\
\hline Negative words & $1.15 a(0.83)$ & $4.00 b(1.99)$ & $1.70 \mathrm{a}(0.89)$ & $4.80 \mathrm{~b}(1.47)$ & $45.72^{\star * *}$ \\
\hline Anger words & $0.30 a(0.44)$ & $2.36 \mathrm{~b}(1.50)$ & $0.29 a(0.33)$ & $1.22 b(1.02)$ & $30.02^{* * *}$ \\
\hline Sad words & $0.30 \mathrm{a}(0.59)$ & $0.42 a(1.12)$ & $0.37 a(0.36)$ & $2.64 b(1.49)$ & $41.44^{* * *}$ \\
\hline Swear words & $0.00 \mathrm{a}(0.00)$ & $0.44 b(0.49)$ & $0.15 a b(0.26)$ & $.50 b(0.54)$ & $19.04^{* * *}$ \\
\hline \multicolumn{6}{|c|}{ Nonverbal Emotional Cues } \\
\hline Vocal Spellings & $1.24 a b(1.37)$ & $1.04 a(0.93)$ & $2.23 b(1.94)$ & $0.67 a(1.03)$ & $18.28^{* * *}$ \\
\hline $\begin{array}{l}\text { Lexical } \\
\text { Surrogates }\end{array}$ & $2.34 \mathrm{ab}(1.88)$ & 2.09ab (1.71) & 2.95a (1.86) & $1.38 b(1.75)$ & $9.09^{*}$ \\
\hline Minus Features & $1.92 \mathrm{ab}(2.40)$ & $1.26 a b(1.43)$ & $2.05 a(2.83)$ & $0.76 b(0.89)$ & $8.08^{*}$ \\
\hline Punctuation & $\begin{array}{c}16.64 a b \\
(9.14)\end{array}$ & $13.61 \mathrm{a}(7.84)$ & $\begin{array}{l}21.27 \mathrm{~b} \\
(11.30)\end{array}$ & 13.11a (8.82) & $11.59^{* *}$ \\
\hline $\begin{array}{l}\text { Grammatical } \\
\text { Markers } \\
\text { Other verbal } \\
\text { cues }\end{array}$ & $0.38 a(0.66)$ & $0.42 a(0.82)$ & $1.32 b(1.30)$ & $0.65 a b(1.01)$ & $17.19^{* *}$ \\
\hline Assent words & $3.06 \mathrm{ab}(1.66)$ & $2.78 a b(1.61)$ & $4.65 a(2.67)$ & $2.28 \mathrm{~b}(1.38)$ & $10.18^{*}$ \\
\hline
\end{tabular}

when I use text-based chat".

More than half of the participants (14/20) reported happiness as the easiest emotion to express, since they can apply different strategies such as emoticons, lol, haha, and punctuations to show their emotions. They also reported that they tend to IM with their friends more when they are happy, so they know how to express happiness and joy to their friends. For example, "it is very easy to joke around, use a 'haha' or 'lol' or emoticons to express happiness while chatting", "BECAUSE YOU CAN DO THISSSS! :D HAPPPPINNNNESSSSSS", or "the use of smiley faces, "lol", and "hahah" helps to easily show joy and ease. Also, usually when I talk to (friend's name) I'm happy so I knew how to tell her I was happy". The rest of participants $(6 / 20)$ reported anger as the easiest emotion to express in their conversations. They reported emotion words, emoticons, and upper case letters as the main cues that make it easy for them to 
express anger. For example, "it's very easy to portray when you're angry and type angry, using different words, visual cues and using all caps."

The answers to the most difficult emotion to express, however, were more diverse. Nine out of twenty participants reported sadness as the hardest emotion to express. Lack of emoticons was one of the reasons they reported. They also reported that sadness is a deep emotion and they usually communicate it through subtle facial cues such as eye expression, which are missing in IM. "For me, sadness is displayed in my face and my eyes. When I'm sad, I don't want to talk about it. So when the only way of communicating my sadness is through message, that makes it difficult to do.", "sadness is a very personal emotion so you have to be around people to feel it", or "I think sadness is the hardest because the only way (friend's name) would know I was sad is if I put a sad face $(:(:)$ ). Seven out of twenty participants reported contentment/relaxed as the hardest emotion to express, since they were in a neutral state and had no extreme emotion to express. The rest of participants, four out of twenty, reported anger as a hard emotion to express. Some of their answers were: "when it comes to deeper emotions like sadness or anger I tend to use a lot of gestures, facial expressions and I seek them in the respondent. Not being able to utilize those aspects of conversation was frustrating", "I like to focus on why I'm angry before expressing my anger", " or "I like to vent anger physically, through sports and lifting weights rather than emotionally".

\subsubsection{Discussion}

Overall, the results of this study provide empirical support for Brunswik's lens model (1956) in synchronous text-based CMC, demonstrating that emotional state of the encoder can affect emotional communication and the usage of text-based cues in IM. 


\subsubsection{Verbosity}

The first interesting point was the number of words per conversation and words per minute participants used among four conditions. Similar to Hancock et al. (2007) participants in all four conditions produced words at approximately the same rate. However, inconsistent with their results that showed participants in negative mood used fewer words compared to a positive mood, we found no significant differences in the number of words per conversation among four conditions. A possible explanation could be the substantially different informal context of our experiment and using participants that knew each other for a quite some time that could talk about positive and negative emotions. Since the number of words per conversation was consistent across conditions, this increased the interest in exploring the relative proportions of the number of cues that were used in different conditions.

\subsubsection{Verbal Emotional Cues}

As expected, in the happy condition, participants used more positive emotion words compare to the other three conditions. Similarly, in the angry condition participants used more angry words compared to the other three conditions, and likewise for the sad mood. Of course, this is partially influenced by the topics of their conversations in those conditions, which were life experiences specifically portraying events that would be described with those words. These results demonstrated that memory elicitation in the second phase of the experiment could maintain users in the emotional states we required.

\subsubsection{Nonverbal Emotional Cues}

Consistent with the Hancock et al. (2007) study, participants used more punctuation in the happy condition than the sad and angry conditions. Participants also used a significantly higher number of vocal spellings (e.g. sooo, weeell) in the happy condition compared to the sad and angry conditions. This result from informal chat aligns 
with a previous task-based study I (Pirzadeh and Pfaff, 2012) in which participants used less vocal spelling during time-pressured conditions that were designed to frustrate participants through frequent task switching. The proportion of manipulations of grammatical markers (alterations of the presentation of words, e.g. all capital letters, strings of periods or commas) were higher in the happy condition than the angry and relaxed condition, but not the sad condition. The proportion of lexical surrogates (e.g. uh huh, haha) and minus features (abbreviations and acronyms) were used significantly higher in happy than sad condition.

Cue categories such as punctuation, vocal spelling, lexical surrogates, and manipulation of grammatical markers are noteworthy in how they attempt to mimic real speech (Hancock, 2004). Participants seem to apply these types of emotional cues to adapt the prosody of face-to-face communication to text-based CMC, especially when they are in a happy mood more than the other three moods of relaxed, angry, and sad. As such, these results extend to CMC the face-to-face findings of Scherer (1978) and Ekman (1982), which showed prosody (e.g. tone of voice, frequency, pitch) is one of the main cues to emotional expression and is exhibited differently across various emotions. These results may have implications for the automatic detection of positive mood, as these groups of emotional cues are relatively easy to capture in real time during a chat conversation.

Another interesting result is the usage of emoticons. Emoticons were defined as symbols that resemble facial expression and body movements and are used very often especially in instant messaging (Derks, Bos, and Grumbkow, 2008). Derks, Bos, and Grumbkow (2008) showed that participants used more emoticons in positive than negative emotional states. The results of this study, however, showed no significant difference in the number of emoticons participants used in four conditions. One possible explanation of this result may be the diversity of emoticons (both positive and negative) 
we had in our experiment, since in their study (Derks, Bos, and Grumbkow, 2008) a limited number of emoticons for negative emotional expression was provided.

Overall, the happy condition led to more use of nonverbal cues than the two negatively-valenced conditions of sad and angry. The manipulation check after watching the video clips confirmed that participants were in the desired mood at the beginning of the chat conversation and it persisted throughout each chat session as shown by the use of significantly different numbers of positive, angry, and sad words. Yet the main nonverbal manifestation of the negative moods (angry and sad) in the chat logs was simply a reduction in the number of nonverbal cues used compared to the happy condition. It suggests that a happy mood promotes an overall increase in nonverbal emotional expressivity in IM, which could be a possible explanation of why most of the participants reported happiness as the easiest emotion to express. This increase in nonverbal emotional expressivity has three potential explanations.

The first is that IM, as it is currently implemented in common desktop and webbased applications such as Google Chat, may not support negative expressivity sufficiently. It may be that letters, numbers, punctuation, and even emoticons are illsuited to express negative emotions. This explanation is in line with the survey results, in which some of the participants reported that sadness and anger are difficult to express in IM because of the lack of cues to communicate facial expression in this medium. This explanation also put SIP theory in a new perspective with respect to the negative emotions, since the results showed that users were not able to find any strategies to convey nonverbal behaviors to express their negative emotions. The second explanation is that participants expressed negative emotions using cues not among those captured in this analysis. Lastly, the third explanation is that perhaps sad and angry partners chatting informally turn their focus inward and become less expressive overall. This explanation is in line with the survey results, in which some participants reported they 
want to focus more on the reason that made them angry before expressing their anger. This is also in line with the results of Pfaff's (2012) study showing an increased inward focus of participants in a negative mood in the NeoCITIES simulation, a six-person team decision-making task. This behavior manifested as reduced attentiveness to their partners.

\subsubsection{Assent words}

Participants used a significantly higher number of assent words (e.g. agree, OK, yes) in the happy condition than the sad condition. Hancock [11] also showed the degree of agreement as one of the main strategies that participants used to express their positive emotion compared to negative emotions in CMC. However, their data showed that it is the frequency of negation words (e.g. not, no, never), rather than the frequency of assent words, which differentiates positive emotion from negative. Our data showed no significant difference in the frequency of negation words in different conditions. A possible explanation for not having significant difference among negation words can be explained by the conversation context of this study. Participants mainly talked about their personal experiences and they might not have had many opportunities to express disagreement, compared to the task-based context in Hancock et al. (2007) study.

To conclude, the main goal of the quantitative analysis was, through a quantitative approach, to see whether pre-defined cues, such as those provided by Boonthanom (2004), would appear in different proportions under different emotional conditions. The advantage of the quantitative approach was the speed and efficiency to mine large data sets, though they only capture content, not context. However, the next phase of analysis took a qualitative approach and applied conversation analysis to preserve the complexity and richness of the content.

\subsection{Results (Qualitative analysis)}


A qualitative analysis in this section focuses more intently on the emotional cues participants used and the reasons behind using those cues in different emotional states and specifically explored if/why IM supports happy emotion more richly than negative emotions of sadness and anger. We focused on conversations only in three mood conditions of happy, sad and angry, since quantitative analysis (previous section) showed that participants were in a neutral state had no extreme emotion to communicate in the relaxed condition.

\subsubsection{Data analysis}

Qualitative data analysis was influenced by the grounded theory approach (Glaser \& Strauss, 1967). The results were grounded in and generated from naturally occurring data collected from 30 conversations in three moods of sad, happy, and angry. Within the grounded theory approach, two methods of data analysis were used in this study: content analysis (Krippendorf, 1980) and conversation analysis (Schegloff, 1987). Content analysis was used to identify emerging themes and categories and also persistent patterns and differences within and across three conditions of sad, happy, and angry. Sequence analysis (Schegloff, 1987; Schegolff, Koshik, Jacoby \& Olsher, 2002) was the specific form of conversation analysis applied in this study. It enables a microanalysis of emotion in interaction by breaking down the data into groups of related utterances and provides insights into how participants express and acknowledge emotion in interaction. In conversation analysis, it is assumed that conversation has structure and the meaning is shaped in the context of the exchange. According to conversation analysis, each conversation occurs in a series of utterance exchanges between two participants of a pair. However, in order for a conversation to be understood as a coherent episode, the exchanges of utterances need to have a structure. Utterances could be grouped in different sequences. A sequence is a group of utterances through which participants accomplish and coordinate an interactional activity 
(Mazeland, 2006). A question followed by an answer, a request followed by a decision, criticism and the reply to it, or a simple comment/inform-acknowledgment are all examples of two-way sequences or adjacency pairs. This study specifically focused on sequence analysis and how emotion is expressed and responded to throughout different sequences.

The following section reports how the overall structure and main topics of conversations were affected by the mood condition. It is followed by exploring emotion expressions and responses through utterances related to each mood.

\subsubsection{Overall Structure and Main Topics of The Conversations}

Conversations in the sad condition mostly started with participants expressing sadness from watching the video clip (a son witnessing the death of his dad), which was followed by topics related to the video such as empathizing with the main character of the movie or questioning what movie the video was from. Anger expression was also part of the beginning of the conversations in the sad condition. Participants got angry about why the young actor gets exposed to his father's body after he died. The most common topics discussed after this initial period were related to participants' sad personal stories, such as losing a friend or family member or sad movies they watched before. Personal stories were either triggered by the video clip, specifically elicited by a partner, or were brought up to empathize with one another's stories. Overall, participants empathized with each other through confirmation cues and similar personal stories, except a few instances of one partner trying to change the mood of the conversation to the baseline mood.

In the angry condition, participants were more engaged in topics related to the video (the bully scene) such as asking what movie the video was from, discussing the main scenes of the movie, how the bully made them angry, how they would react if they were the main character of the movie, and the reasons behind the bully scene of the 
video clip. Related personal stories were also discussed, but not as often as in the sad condition. Compared to the sad condition, where participants tried to empathize with each other by talking about similar sad experiences in their lives, in the anger condition participants tended more to restore the mood of conversation to the baseline mood by making jokes and teasing.

In the happy condition, more diverse topics were discussed than in the sad and angry conditions. Participants mostly started their conversations with expressing their positive feeling about the video (funny clips of babies) and talking about different parts of the video that were more interesting to them. After this initial period, participants tended to sustain the happy mood of conversation by talking about a diverse range of topics from their feeling about having babies and funny stories from childhood to telling jokes and making fun of each other.

\subsubsection{Emotion in interaction}

The following sections explore how emotion was expressed and responded to in different sequences of utterances. Emotional cues participants used to express their emotions throughout the conversations and strategies they used to provide a response to their partner's emotional comments were identified at this stage. Figure 8 shows an overall view on different emotional cues participants used in the emotional expression and feedback.

\subsubsection{Emotional Expression}

Participants used emotional words as the main strategy and lexical surrogates and emoticons as supporting strategies to express their emotions. 


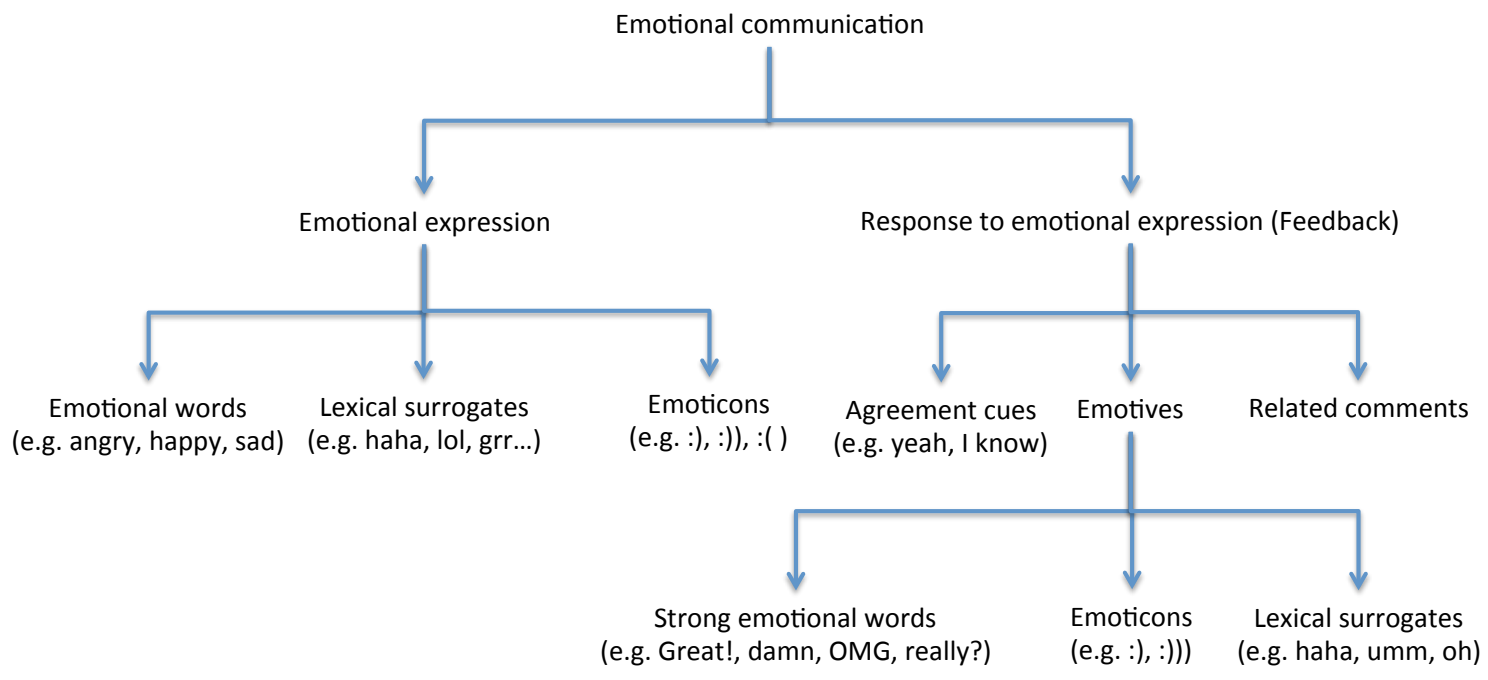

Figure 8. Emotional cues used in emotional communication

\section{Emotional words}

The main strategy that participants used to express their emotion in the utterance was using emotional words to describe their emotion. In sad expression, sad words were used such as sad, crying, bawling, poor, tough, depressed, downer, not liking, tearing up, and awful. For example, "he kept crying and it made me sad again", "poor him and the champ!", "Well that was a downer.", "Holy depressing.", "that sucks", "i always have nightmares about my dad dying so idk that stuff gets me", "it sucks to lose a grandparent your close to.", "i'm sad the guy died but im more sad for the kid.", "It doesn't get sadder than that my dude."

In the happy expression, happy words were used such as like, hilarious, love, laugh, funny, favorite, and adorable. For example: "that was my fav clip!", "Hands down best movie ever!", "It was just so adorable and funny.", "I love his laugh.”, "i love babies", "it was hilarious", "He is the funnies little dude ever.". 
Angry words such as horrible, hate, angry, mad, annoying, pissed off, and hell, or epithets such as mean, stupid, and loser were used in anger expression: "omg like i hate how everyone just stood around except for like one kid", "i get really mad at hypocrites. they make me want to scream", "it was so annoying", "Those make me angry.", "what a jerkface".

\section{Lexical surrogates}

Lexical surrogates, mostly at the end of utterances, were used for emotional communication to mimic real speech and communicate vocal sounds. Lexical surrogates are textual representations of vocal sounds that are not words, such as "uh huh", "haha", and "oh". "lol" (laugh out loud) was also considered to be in this category, since it is a textual representation of vocal sound of laughing out loud.

No lexical surrogates were used in sad expression. In anger expression, only one instance of "grr..." was found: "that happened to me a little in 5th grade. I had no friends in 5th grade and kids would like lick my pencil and give it back to me because they knew i would get grossed out....grrrrrr". In happy expression, however, lexical surrogates of "haha" and "Iol" were repeatedly used. For example, "yay babies lol", "i think its worth it. they are a little bundle of emotions haha", "i loved the first one and the one who liked the ripped paper. they were my favorites lol", "And then you get all the fun parts of a baby but not the hard parts. Lol", "all kids are weird in a gap lol", "they all laughed like old men lol".

\section{Emoticons}

Using emoticons at the end of utterances was another strategy to communicate emotion by mimicking facial expression. Emoticons are pictographs constructed from punctuation and letters, such as :-( for a sad face, or :-D to indicate laughing in IM. Emoticons were only used in the happy and sad expression and not in anger expression. Five total instances of ":(" and ":[" were the only emoticons that were used in sad 
expression. In the happy expression, 11 different types of emoticons were used, including “:D”, “;-)", “:)” , “:]” , and “:)))”. Some examples of using emoticons as a supporting tool for emotional expression are as follows: "i started crying :( " "but i feel bad for my papa because now he's the last one left in his family :( ", "my stomach really hurts :( ", "the first time i saw home alone 2 in the movies i peed my pants :D", "but when I like 4 laughing I was thinking how would it be if 4 start crying together ;-)" , "i sometimes imagine things and laugh :D”.

\subsubsection{Response to emotional expression (feedback)}

Feedback can express the receiver's interpretation of the prior utterance and can provide a means for making sure everyone has the same understanding of what has been said. The previous sections explained what strategies participants used to express emotion in their utterances throughout their conversations. This section specifically focuses on feedback and explores how participants responded to their partners' emotional expression.

Based on the data analysis, three main response categories were identified including agreement/confirmation cues, emotives (strong emotional words, lexical surrogates, and emoticons), and related comments (personal opinions, experiences, and stories).

Agreement cues

The first category of feedback identified from the conversations was agreement/confirmation words or phrases such as "I know", "right", "yeah", or "agree”, which are called agreement. This category of cues was used to show the receiver agreed and empathized with sender's utterance and emotional expression. For example:

A: Well that was a downer.

B: Yea... 
A: the one that fell over was sooo cute

B: i know!!!!

A: i hate how some men are so violent. I just really dont like violence, it is truely never the answer

B: i agree.

\section{Emotives}

The second category of feedback, emotives, included three subcategories: strong emotional words (e.g. "oh my god", "damn”, "holy crap", "what?”, "really?", "outstanding!", "great"), lexical surrogates (e.g. "oh”, "Awe”, "haha”, "Iol”), and emoticons (e.g. “:(“, “:)))”, “:D”). Emotives were used alone, in a group, or in combination with agreement cues.

A: his wife got cancer like....10 years down the line and they only caught it when she had like 3 months left

$A$ : and i went to her funeral

B: damn........

$A$ : the first time i saw home alone 2 in the movies i peed my pants :D

$\mathrm{B}$ : whaaaaat?

B: hahahahahahahaha

A: the one kid that was laughing and then sort of fell over, that was really cute

$\mathrm{B}$ : hahaha yeah

Table 6 shows the list of most frequent agreement cues and emotives participants used in three conditions of sad, happy, and angry.

\section{Related comments}

The third category of feedback was identified as related comments, which include any comment related to the sender's utterance such as the receiver's personal opinions, experience, or stories. Personal stories were mostly used in the sad condition in response to sad personal stories that participants talked about. 
Table 6. Table Agreement cues and emotives in three conditions of sad, happy, and angry

\begin{tabular}{|c|c|c|c|c|c|c|c|}
\hline Feedback & Cue & Sad & $\begin{array}{l}\text { Percentage } \\
\text { from the } \\
\text { total } \\
\text { feedback } \\
\text { cues }\end{array}$ & Angry & $\begin{array}{l}\text { Percentage } \\
\text { from the } \\
\text { total } \\
\text { feedback } \\
\text { cues }\end{array}$ & Happy & $\begin{array}{l}\text { Percentage } \\
\text { from the } \\
\text { total } \\
\text { feedback } \\
\text { cues }\end{array}$ \\
\hline \multirow{8}{*}{ Agreement } & yeah & 48 & 0.32 & 36 & 0.26 & 19 & 0.11 \\
\hline & right & 8 & 0.05 & 5 & 0.03 & 4 & 0.02 \\
\hline & I know & 12 & 0.08 & 6 & 0.04 & 7 & 0.04 \\
\hline & agree & 4 & 0.03 & 5 & 0.03 & 1 & 0.00 \\
\hline & TRUE & 1 & 0.00 & 4 & 0.03 & 3 & 0.01 \\
\hline & Exactly & 0 & 0 & 3 & 0.02 & 1 & 0.00 \\
\hline & $\begin{array}{l}\text { me too/me } \\
\text { neither/same/ }\end{array}$ & 10 & 0.07 & 4 & 0.02 & 7 & 0.04 \\
\hline & Total & 83 & 0.55 & 63 & 0.45 & 42 & 0.24 \\
\hline \multirow{4}{*}{$\begin{array}{l}\text { Strong } \\
\text { emotional } \\
\text { words }\end{array}$} & OMG & 13 & 0.08 & 4 & 0.02 & 8 & 0.04 \\
\hline & really?/seriously/ & 4 & 0.03 & 5 & 0.03 & 5 & 0.03 \\
\hline & $\begin{array}{l}\text { e.g. outstanding, } \\
\text { great, damn }\end{array}$ & 11 & 0.07 & 4 & 0.02 & 18 & 0.10 \\
\hline & Total & 28 & 0.18 & 13 & 0.09 & 31 & 0.18 \\
\hline \multirow{8}{*}{$\begin{array}{l}\text { Lexical } \\
\text { surrogates }\end{array}$} & oh & 7 & 0.05 & 16 & 0.11 & 6 & 0.03 \\
\hline & awe & 0 & 0.00 & 3 & 0.02 & 2 & 0.01 \\
\hline & wow & 0 & 0.00 & 2 & 0.01 & 1 & 0.00 \\
\hline & ummm & 9 & 0.06 & 1 & 0.00 & 9 & 0.05 \\
\hline & haha & 10 & 0.06 & 19 & 0.13 & 56 & 0.32 \\
\hline & Iol & 2 & 0.01 & 2 & 0.01 & 7 & 0.04 \\
\hline & $\begin{array}{l}\text { Other (e.g. Eh, } \\
\text { ahh, ew) }\end{array}$ & 6 & 0.04 & 14 & 0.10 & 14 & 0.08 \\
\hline & Total & 34 & 0.22 & 57 & 0.41 & 95 & 0.55 \\
\hline Emoticons & $:),:)), \ldots$ & 5 & 0.03 & 5 & 0.03 & 4 & 0.02 \\
\hline Total & & 150 & & 138 & & 172 & \\
\hline
\end{tabular}

Related comments were used with and without agreement cues and emotives and could be considered to be the emotional expression in response to the most recently discussed topic. For example:

A: i hate when bad stuff happens to kids in movies

$B$ : $i$ hate when bad things happen in general

A: ever since I was little I always wondered what it felt like to die, but I didn't want to stay dead

B: I know! I used to run into my parents room at night and cry because I was thinking about death 
A: like the one with the baby and ripping the paper

$B$ : haha I liked the one that is most viewed on youtube

A: you know the girl at iu that died at a little 5 party? that was my friends roomate and i didn't even know it. she was sleeping when her friend died. i know its gonna take her a long time to get over it B: : ( awh i didnt know that

\subsubsection{Emotional prosody (capital letters, punctuation, and vocal spelling)}

In both emotional expression and response, participants used different strategies to adapt the prosody of face-to-face emotional communication to instant messaging, such as altering the presentation of words using capital letters and punctuating statements using strings of exclamation or question marks to emphasize certain words in a statement or express the intensity of their emotion. They also used vocal spelling to mimic a specific vocal inflection and ellipsis to indicate a pause or silence.

Participants used capital letters and strings of exclamation or question marks to mimic vocal intensity and loudness of specific words or phrases. These two strategies were mostly used to express excitement, joy, surprise, and anger. For example: "O.M.G. FOUR babies" (happy), "I can't even IMAGINE" (happy), "so WTH movie was THAT?" (angry), "I HATE BULLIES" (angry), "GO HELP LOSERS" (angry), "OMG THAT WAS ADORABLEEEEE" (happy), "OMG I STILL CRY!" (sad), "THAT WAS SO FREAKIN SAD", (sad)", "right?!?!?!" (sad), "like elementary kids can be evil!!!" (angry) , "i love pretty little liars more!!!!” (happy).

Another strategy used to mimic the prosody of face-to-face emotional communication in IM was altering of spelling to mimic a specific vocal inflection. For example: "sooooooo glad the internet was not a big thing back then" (happy), "i laughed and i'm suuure people thought i was nuts" (happy), "freaking cuuute" (happy), "they were soooo cute" (happy), "you loooooooove k names" (happy), "interrrrrrrresting" (happy), "its sooo sad" (sad), "like i thought my sister's keeper was soo0o0000000 sad " (sad), "i 
so000000 did not bully that kid but they still suspended me" (angry), "i was like why are you friends with a bully? whhyyy?" (angry).

Participants also used ellipsis "..." to indicate pause of silence or unspoken words. For example: "god...so miserable" (sad), "damn......." (sad), "I don't get embarrassed by much.... did fall up the stairs with my hands in my pocket once", "haha...way to go!!!" (happy).

\subsubsection{Beyond Adjacency Pairs}

Adjacency pairs were not the only type of sequence throughout the conversations. Three, four, and five-way sequences were also identified which mostly consisted of using agreement cues and emotives back and forth. For example:
A: she kills herself
B: holy crap
B: SUPER SADDDDDDNESSSS
A: i KNOWWWWWW
A: OK. Hands down best movei ever!
B: FOR REALS. Completely agree.
A: Hahaha! So hilarious!
A: we had to sit down and laugh
B: LOL
A: silly me
B: oh my god
$\mathrm{B}$ : hahahahaha
A: O.M.G. FOUR babies
B: RIGHT?!!?!?!?
A: YIKES
$\mathrm{B}$ : haha
A: hahaha :)

\subsubsection{Discussion}

The results of this study, including the level of participants' satisfaction in communicating their emotion (5.9 out of 7 according to the prior quantitative analysis), presented more evidence to support the SIP theory in which individuals adapt different textual cues to communicate their emotion via IM. However, the results of the 
quantitative analysis put SIP theory in a new perspective with respect to the negative emotions, since sadness and anger are more challenging for users to express.

Considering emotion expression and response separately was a new perspective taken in this study. Firstly, in emotion expression, users mainly used words to explain how they feel and why they feel so. However, they used different strategies such as lexical surrogates and emoticons to support their uttered emotion. As expected, this result shows that verbal cues including emotional words play the main role in emotional communication in IM, and other strategies such as emoticons and lexical surrogates are used as tools to support expressing emotion in their utterances. Lexical surrogates and emoticons were mainly used for two purposes. First, to emphasize the emotion of the utterance, where the emotion of the cues (lexical surrogates and emoticons) was the same as the emotion of the utterance for example: "yay babies lol" or " it made me sad :(“. Second, they were used to convey teasing or joking, where the positive lexical surrogates and emoticons were used at the end of statements that did not convey necessarily positive emotion. For example, "all kids are weird in a gap lol”, "And then you get all the fun parts of a baby but not the hard parts. Lol”. Overall, lexical surrogates and emoticons were used at the end of the statement to convey the tone of voice or facial expression.

The limited emoticons usage (16 instances across all three conditions) in the conversations was unexpected. In their study, Walther and D'Addario (2001) found little impact of emoticons in messages that include attitudinally rich verbal statements. Therefore, one possible explanation for the limited usage of emoticons could be that participants used rich emotional statements that didn't need to be emotionally emphasized by emoticons. Another possible argument could be that some participants might have been new to Google chat and not comfortable finding and using the emoticons. Yet another possible explanation could be that IM users replaced "haha" and 
"lol" instead of emoticons since they do a similar job as emoticons and may be easier to type.

In response to emotional expression, participants used agreement cues, emotives (strong emotional words, lexical surrogates, and emoticons), and related comments, or a combination of them. Agreement cues and emotives were used in two ways. They were used alone to indicate acknowledgement in a way that could be considered to be back channeling, in which the receiver provides quick feedback without claiming the floor or taking a turn in the conversation. They were also used in combination with related comments in a way that mimics turn-taking in face-to-face communication since the receiver takes the floor and expresses himself on the topic that the sender just talked about.

The results showed the strong role of short cues, specifically agreement cues and lexical surrogates, in responding to emotional expression. The main strategy receivers used to acknowledge emotional expression and empathize with senders in the sad and angry conditions were agreement cues (specifically "yeah" and "I know") and in the happy condition was lexical surrogates (specifically "lol" and "haha"). One possible explanation for the high number of agreement/confirmation cues is that data consisted of free conversations and not task-oriented dialog. Feedback was mainly used by receivers to agree or empathize with the senders' message. Tagliamonte and Denis (2008) also identified "haha" and "Iol" as the highest frequency cues used by their participants in the flow of conversation as a signal of interlocutor involvement or feedback. They obtained this result after searching and documenting a range of linguistic phenomena in a corpora involving 72 teenagers and over a million words of natural, unmonitored IM.

In both emotional expression and response, participants used capital letters, punctuation, and vocal spelling to adapt the prosody of face-to-face emotional communication (high pitch, prolonged duration, and intensity) to IM. In their study, 
Sasaki and Ohbuchi (1999) compared emotional interaction in CMC and vocal face-toface communication and their results showed that CMC could evoke emotions as intensely and easily as vocal communication. In the vocal condition, however, angry emotions and perceived negative intents prompted aggressive responses, while these effects were absent in CMC (Sasaki \& Ohbuchi, 1999). Therefore, overestimating or underestimating emotional states may happen in $\mathrm{CMC}$, which could lead to inappropriate reactions. Based on the results of the present study, participants often replied to anger expression of their partners with joking and teasing, which might have been caused by receivers underestimating the senders' anger.

Overall, the results of the present study show three main strategies participants used to adapt emotional expression and response to IM: words (emotional words and agreement cues), mimicking the prosody (including lexical surrogates, capital letters, punctuation, vocal spelling) and emoticons. Initial studies on the impact of facial affect in face-to-face communication indicated that facial expressions have greater effects and provide more consensual and accurate judgments of emotion than vocal nonverbal cues (Burgoon, Buller, \& Woodall, 1996). Critics of those studies pointed out, however, that the studies involved unnatural experimental stimuli. Further natural investigation found that vocal expressions could be even more important predictors of emotions than facial expressions in everyday life (Planalp, 1998; Fridlund, Ekman, \& Oster, 1987). Based on the results of the present study, using different strategies to mimic the prosody of speech provides some evidence that supports the importance of vocal cues over facial expression in the context of informal conversations via IM when the visual and aural nonverbal cues are missing.

Later research by Scherer (2003) showed there are some major differences between facial and vocal expression with respect to their recognition accuracy scores. For example, joy is easily recognized in the face, while sadness and anger, followed by 
fear, are generally best recognized in voice. The results of the prior experiment in which the data in the present study was generated showed that participants had more challenges communicating their sadness and anger compared to happiness. The lack of strategies to mimic the voice in anger and sadness in IM communication could be a possible explanation for challenges users reported in communicating their anger and sadness. Although users already developed some strategies, they might not be enough to fully satisfy sadness and anger communication.

\subsection{Conclusion}

This study explored how college friends actually communicate their emotion during informal conversations in instant messaging through a mixed methodology. The quantitative analysis specifically investigated how different emotional states influence cues individuals apply in instant messaging. While qualitative analysis investigated emotion in interaction and identified emotional cues IM users used in emotional communication including emotional expression and response via this medium.

The results of this study provide further empirical support for Brunswik's lens model (1956) in synchronous text-based CMC, demonstrating that the emotional state of the encoder can affect emotional communication in IM. This model can be used as a framework for future studies to explore how unique patterns of emotional cues in addition to other factors (such as personal traits, culture, or social relationships) can characterize different emotional states in IM. Understanding the usage patterns of emotional cues has implications for future research on emotion communication via CMC, as well as for the design of the next generation of IM tools that can facilitate a wider range of emotional expression.

Overcoming the limitations of prior work on emotion expression in text-based CMC (Hancock, Landrigan, and Silver, 2007; Walther, Loh, Granka, 2005) that used role-playing to make users express their emotions, this study explored the topic by 
cultivating emotionally-laden situations, in which participants were more likely to engage in natural emotional conversation, by using video clips and memory elicitation techniques. Future studies need to apply other creative techniques to increase the emotion expression in natural conversation among friends. An alternate approach is to acquire a large natural corpus of IM data and use content analysis to classify conversations into appropriate emotional categories to then be analyzed for verbal and nonverbal cues. Continuing with the need to better understand IM users, prior work demonstrates the importance of considering individual personality traits when assessing communication behaviors (Whittaker, 2003).

Based on the results of study I, extraversion (Scherer, 1978) and emotional intelligence (Petrides and Kokkinaki, 2007) could be the most likely factors to explain some of the variation between individuals in their use of verbal and non-verbal cues to express and respond emotion in $\mathrm{IM}$, and should be considered in future research in this area. Future studies also need to consider that emotional or social cueing (verbal and nonverbal) is not the only mechanism that connects people together in text-based CMC. Whittaker (2003) explains different cognitive cues such as turn taking, availability, shared attention, and interactivity are additional types of cues that facilitate expressive communication. Nardi (2005) goes beyond cognitive and social cueing to discuss social connection and different categories of activities for social bonding (affinity, commitment, and attention) that need to be considered in any type of communication, including textbased $\mathrm{CMC}$ in the absence of traditional nonverbal cues. Longer-term goals are to study text-based CMC beyond emotional expression to explore different categories of cues and activities that individuals use to connect and develop social bonds.

The relatively small sample size (ten couples) was one limitation of this study, though the within-subjects design across four conditions produced forty conversations total, an ample amount of data. Significant results found in this sample shows that this 
topic merits further consideration and future studies need to re-examine these findings with chat data from a bigger sample. The higher number of female compared to male participants is another limitation of this study. Although Hancock et al. $(2007,2008)$ found no gender affect on emotion expression and detection in instant messaging; future studies should reexamine the results of this study in a more diverse participant pool (in terms of gender, age, and ethnicity) than this overwhelmingly young female sample. Also, this study focused on data in English. Future studies should reexamine the results of this study in other languages and cultures.

Regarding to qualitative analysis, this study examined a few aspects of conversation analysis, which were overall structure of conversations, sequence organizations, and lexical choice. There are other areas of conversation analysis such as turn taking, interactional asymmetries and examining the level of asymmetry (e.g. participation, and knowledge) in social interaction that future studies could explore in informal IM communication.

The results of this study were applied as a strong research foundation for the following design study to develop solutions to support emotional communication in IM by facilitating effective and transparent emotion expression and response in instant messaging. 
Chapter 6. Design process

\subsection{Introduction}

The results of previous studies I, II and III as upfront research provided helpful insights to ground, inform, and inspire the design process in this chapter. The design process involved an active process of ideating, iterating, and critiquing potential multitouch gesture based design solutions to support emotional expression and response in instant messaging.

Narrowing down the scope of the design, this study specifically focused on mobile instant messaging (text messaging) and explored multi-touch gesture based design solutions to support emotional communication via this platform. Like desktop instant messaging, text messaging represents a merging of written and oral communication modes. However, the mobile and highly interactive nature of texting sets it apart from desktop instant messaging. The increasing trend of smartphone users has directly affected the number of mobile messaging app users. The reason behind the increasing trend is that the proliferation of cell phones with full keyboards and touchscreen function has made it easier to send mobile instant messages.

This chapter presents different stages of the design process. Brainstorming design sessions were conducted to explore different multi-touch gestures, body icons and symbols related to sadness and anger expression and response. Based on the results of brainstorming sessions and upfront research (study I, II and III), through prototyping and co-design sessions, two main multi-touch gesture based design solutions to support emotional communication in mobile text messaging were created, iterated and evaluated.

\subsection{Exploratory brainstorming design sessions}

The design process was started by conducting two brainstorming design sessions to explore different multi-touch gestures that could be used as input for 
emotional expression and acknowledgement from the sender's point of view (See appendix $E$ for the brainstorming session plan). Expression and acknowledgment were explored separately, since in the study III (conversation analysis) they were identified as two main components of emotional communication in IM. Different body icons and sign/symbols were also explored as the output to be seen from the receiver point of view. These channels were inspired from different channels of emotional communication in face-to-face interaction. Face icons were not explored in the brainstorming sessions, since a wide range of face icon emoticons is already available to represent facial expression. In the quantitative analysis in study III, sadness and anger were identified as emotions that were more challenging to be communicated in IM than happiness. Therefore, the design process started with focusing on sad and angry emotional communication.

\subsubsection{Participants}

Overall 16 visual communication design students participated in two brainstorming sessions, 8 students in 4 pairs for each session. Participants were fourteen females and two males, age range of 20-32, with a mean of 25.4 years. Four participants were graduate students, six juniors and six seniors. Design students were selected for this stage of the design process to make sure that they had enough design skills to communicate their ideas through drawing.

\subsubsection{Procedure}

Each session consisted of four parts of anger expression, anger response, sad expression, and sad response. Participants were paired up in four teams in each session (Figure 9) and asked to write an angry memory they had on the paper form given to them (See appendix $F$ for all the forms were used in the brainstorming session). This memory elicitation technique (Moris, 1989) was used to elicit anger and put participants in a specific emotional context. Then they were asked to draw eight multi-touch gestures 
to express their anger. They used painting colors to draw their gestures. They were also asked to draw as many body icons and sign/symbols as they would like to show their anger to their friends. On the forms they were given, they had space for four body icons and four symbols. However, they were given a blank sheet to be able to draw as many ideas they could generate. Considering the time limitation for the session (1.5-2 hrs.), participants were given 30 seconds for each part of the gesture, body icons, and symbols creation. The significance of the announcement was that it ensured everyone knew that the agreed upon goals must be met by a certain time. This encouraged them to stay on track and generate as many ideas as possible within the allotted timeframe.

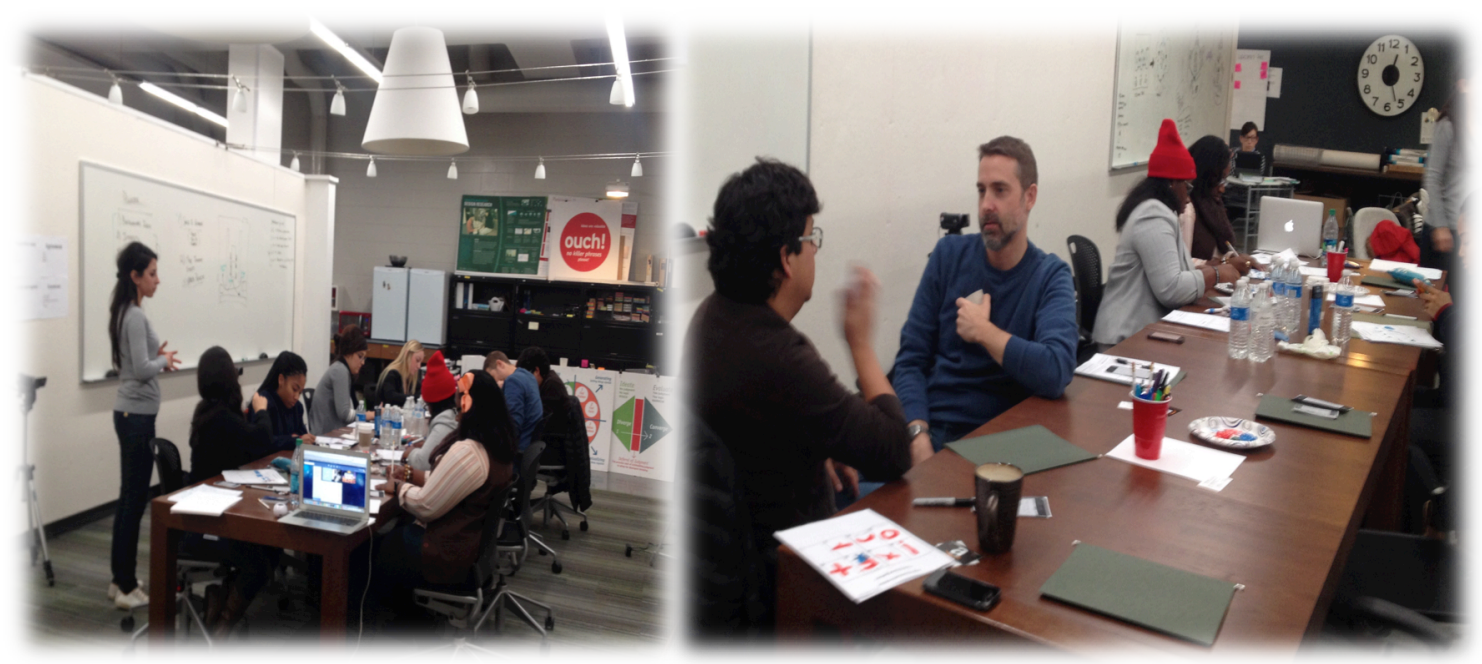

Figure 9. Brainstomring sessions setting

Then they were asked to switch their memory forms with their team member and read through each other's messages. They were asked to explore eight multi-touch gestures they would use to acknowledge their friend's anger expression and also four body icons and four symbols they would like their friends to see on the other side. All the 
above steps were repeated for the sad expression and response. Note that as a brainstorming session, it was emphasized that quantity was more important than quality.

Then they were asked to switch their memory forms with their team member and read through each other messages. Then they were asked to explore eight multi-touch gestures they would use to acknowledge their friends anger expression and also four body icons and four symbols they would like their friends to see on the other side. All the above steps were repeated for sad expression and response. Note that, as a brainstorming session, it was emphasized that quantity was more important than quality.

\subsubsection{Results}

For every category of anger expression, anger response, sad expression and sad response, an affinity diagram was created, and different ideas for each channel of multi-touch gestures, body icons, and symbols were grouped in different themes.

\subsubsection{Anger expression}

In total 113 multi-touch gestures were created for anger expression. The same multi-touch gestures were grouped together and placed into distinct categories, and the five most frequent gestures for anger expression were identified (Figure 10).
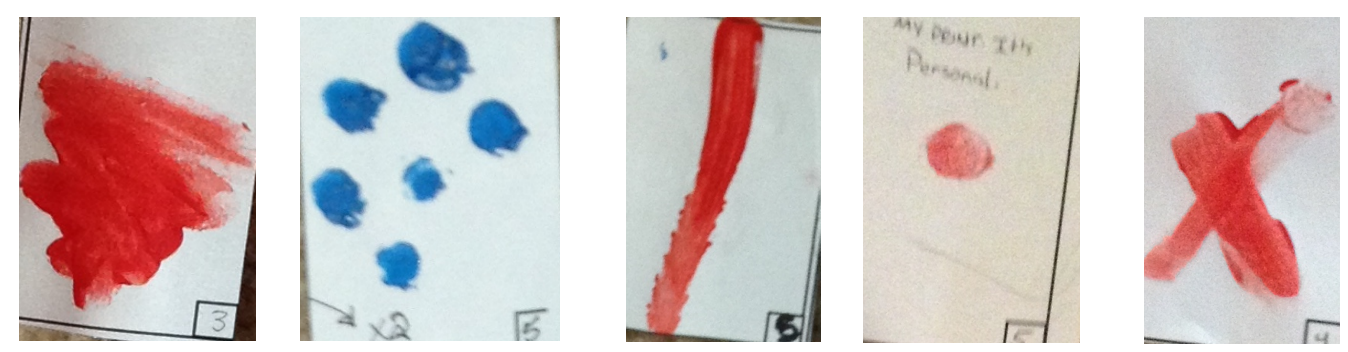

Figure 10. Anger expression- multi-touch gestures

Body icons (in total 56 icons) were also categorized in three main categories. The first category identified was static body expression. This category of body icons 
visualizes the angry static body expression, for example shaking hands in the air or pointed down from anger. The second category of body icons for anger expression presented physical anger such as hitting or kicking. The third category was body icons that presented pulling out hair. Figure 11 shows a few examples of body icons in each category.
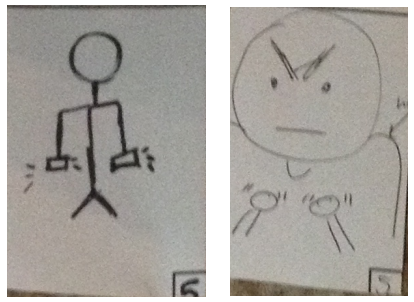

Static anger expression

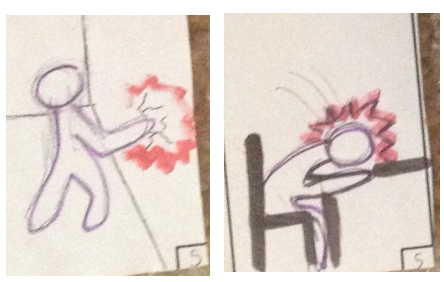

Physical anger
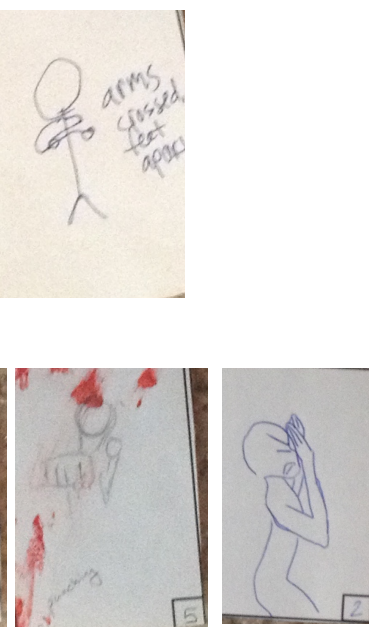

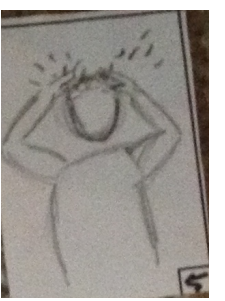

Pulling out hairs
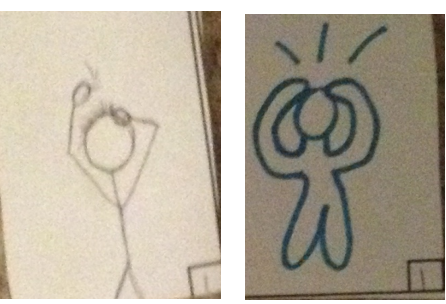

Figure 11. Anger expression- body icons

The same anger expression symbols (in total 54 icons) were also grouped.

Figure 12 shows the five most frequent symbols that were created in the brainstorming sessions.
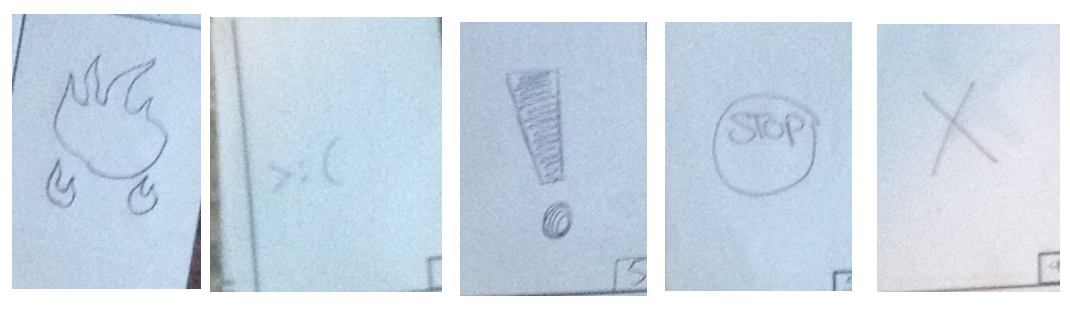

Figure 12. Anger expression- symbols 


\subsubsection{Anger response}

Data analysis was also done for anger response multi-touch gestures, body icons, and symbols. 102 multi-touch based gestures were gathered. Figure 13 shows the five most frequent multi-touch gestures participants created for anger response.
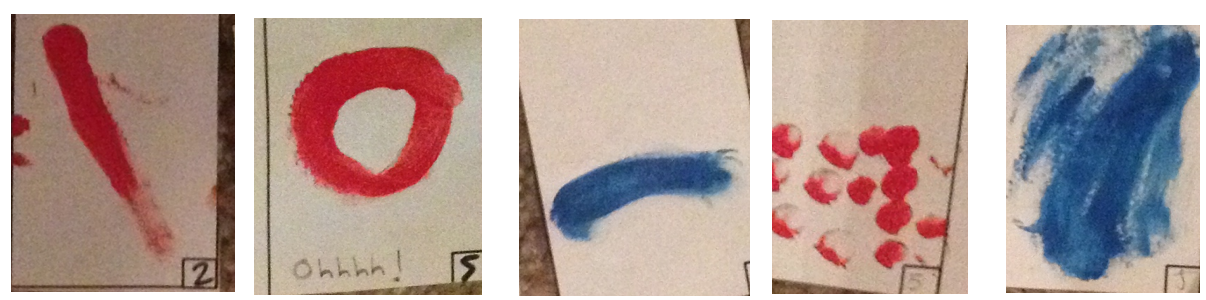

Figure 13. Anger response- multi-touch gestures

As figure 14 shows, five main categories were identified from the body icons (in total 45 icons) generated for the anger response. The first category of icons visualized anger in response to anger. Icons in this group showed that when their friend is angry, they would get angry too. The second category visualized how recipients get sad when their friend is angry. In the third category, they were shocked or surprised from their friends' anger. The fourth category visualized comforting and empathy with their friends. There was also a fifth category where they might also laugh at their friends' anger.

The anger response symbols (in total 49 icons) were also categorized, and four main categories were identified (Figure 15). Interestingly, category themes identified for symbols were similar to body icon categories. Participants visualized sadness, anger, shocked and comforting through symbols in this part. 


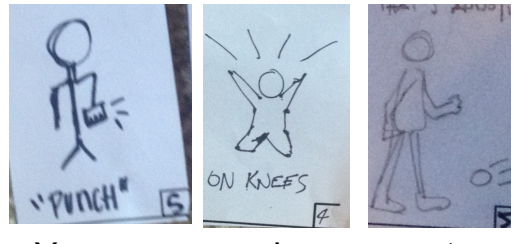

You are angry, I am angry too

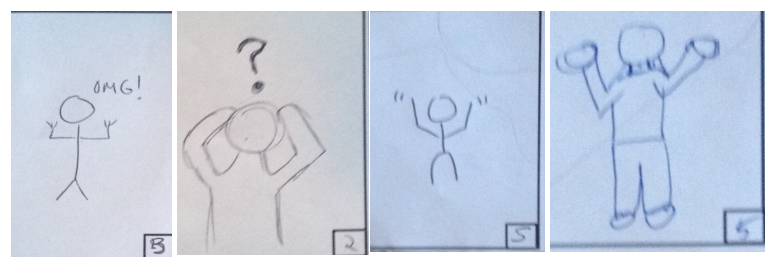

You are angry; I am shocked/surprised
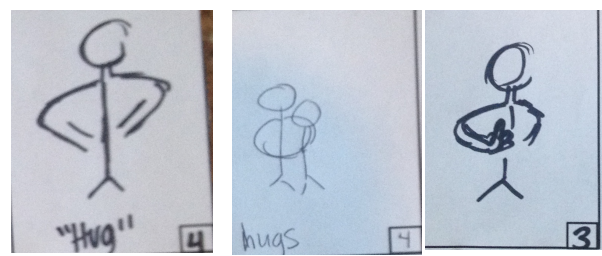

You are angry; I comfort you
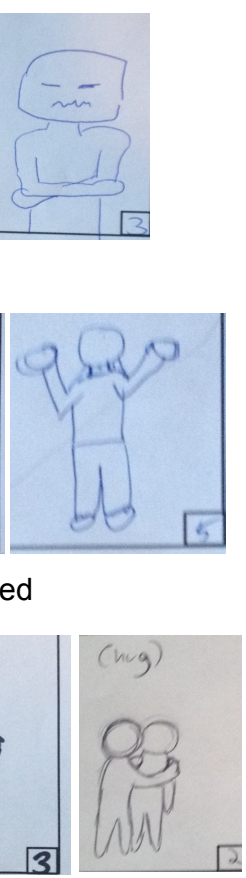

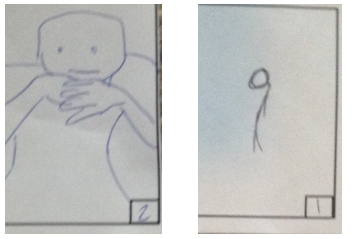

You are angry, I am sad

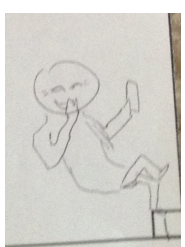

You are angry and it is funny

Figure 14. Anger response-body icons
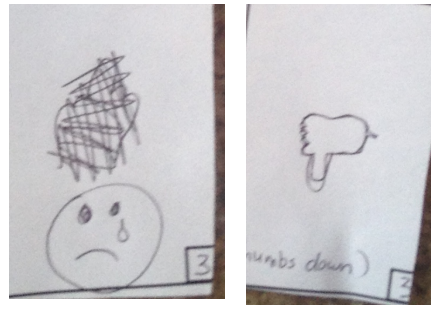

You are angry; I'm sad

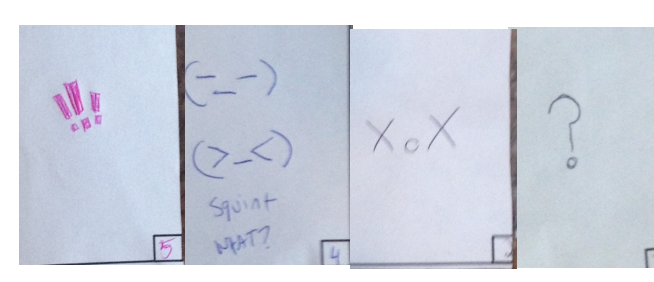

You are angry; I am shocked

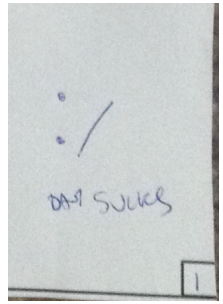
(1) 


\subsubsection{Sad expression}

The same process of data analysis was used to categorize multi-touch gestures, body icons, and symbols for sad expression and response. In total 104 multitouch gestures were collected for sad expression. Affinity diagrams were created, and identical multi-touch gestures were grouped together. Below are the five multi-touch gestures that were created with the highest frequency.
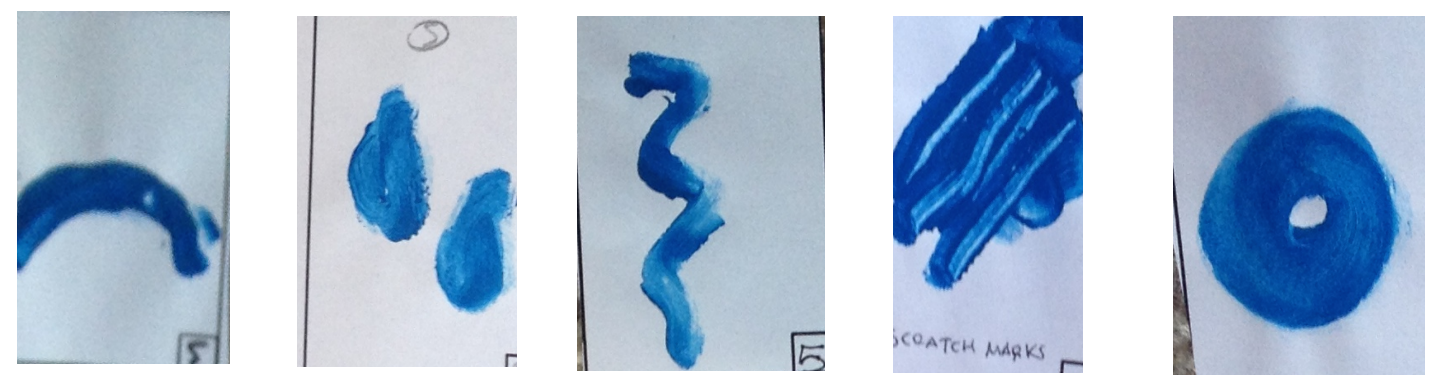

Figure 16. Sad expression- multi-touch gestures

Sad expression body icons (in total 54) were categorized into four groups. Body icons that showed slouching, tearing, and wiping off tears, sitting alone, and laying down. Figure 17 shows two icons from each category.

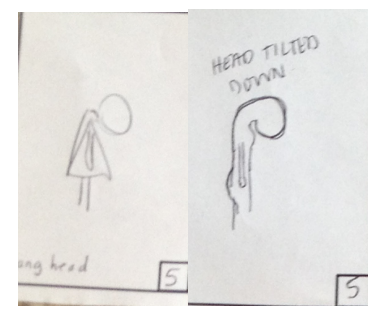

Slouchin

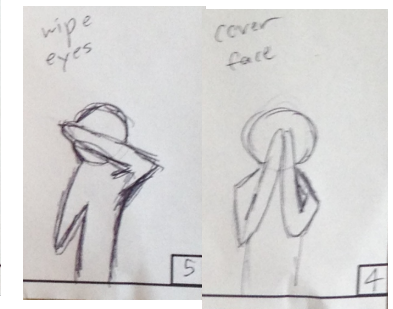

Tearing and wiping off

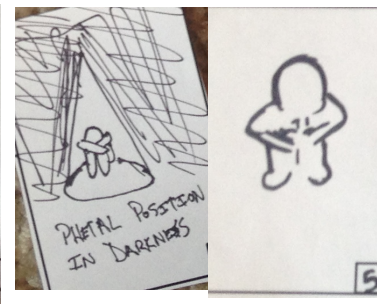

Sitting

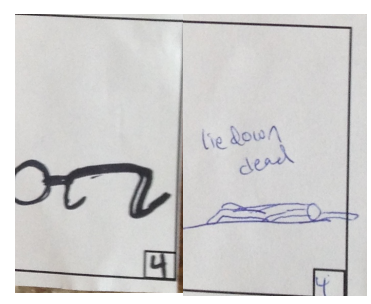

Laying

Figure 17. Sad expression- body icons

Sad expression symbols (in total 39 icons) were also categorized and below are the four most commonly generated symbols for sad expression. 

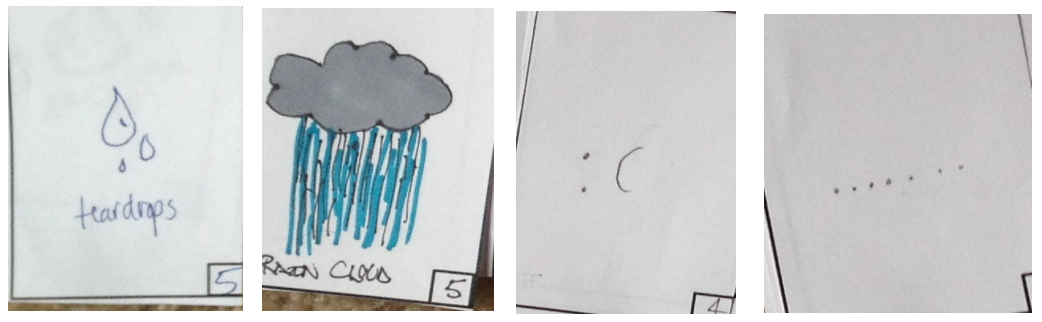

Figure 18. Sad expression- symbols

\subsubsection{Sad response}

The 106 multi-touch gestures created for the sad response were also categorized. Below are the five gestures with the highest frequency.
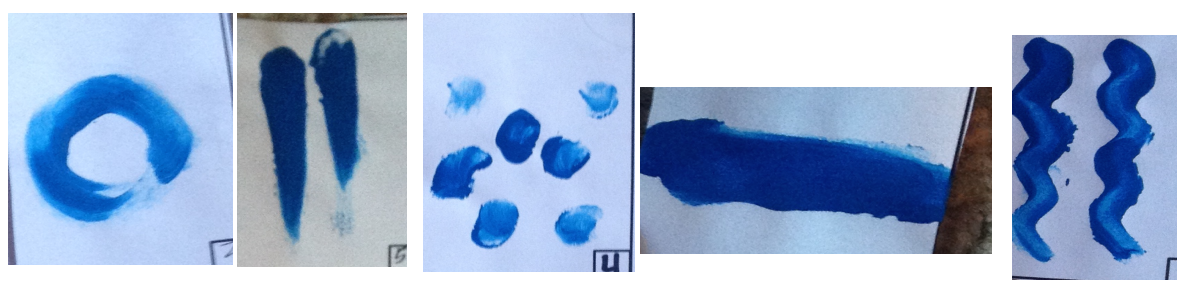

Figure 19. Sad response- multi-touch gestures

Sad response body icons and symbols were also grouped. Sad response body icons (in total 45) were categorized into three categories of sadness, being shocked/surprised, and comforting. Figure 20 shows three samples for each category.

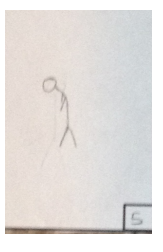

You are sad; I'm sad

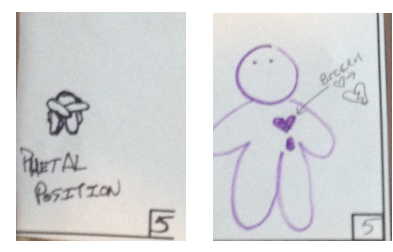

You are sad; I comfort you
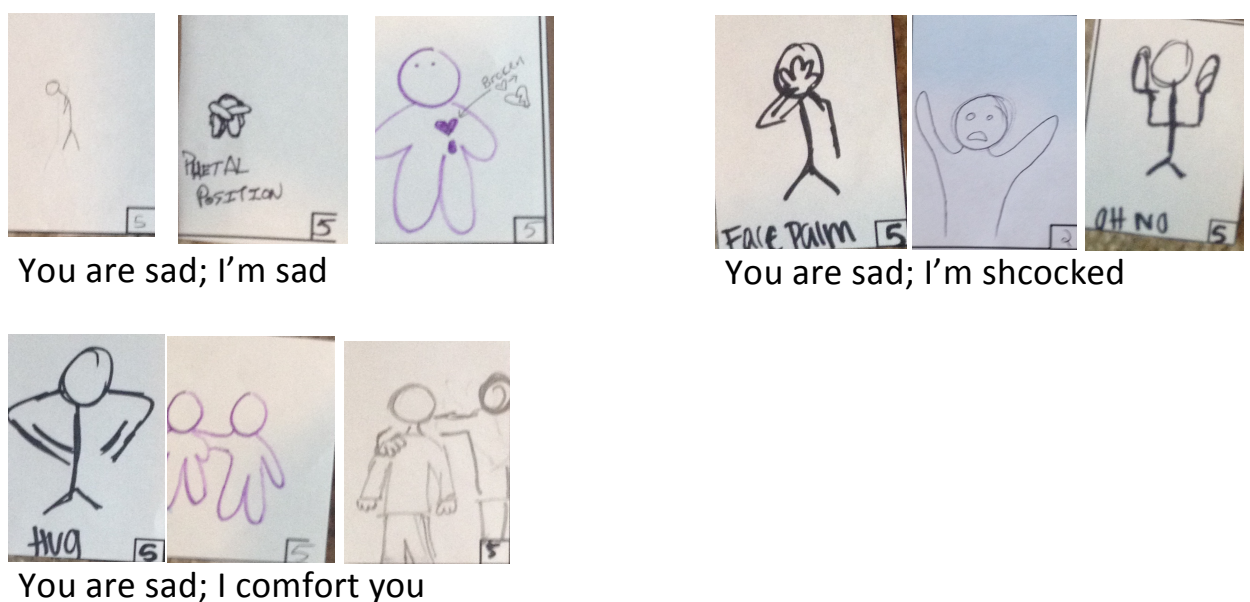

Figure 20. Sad response- body icons 
Sad response symbols (in total 42) were categorized into two main categories that represent sadness in response to sadness and also comforting that included hugging or giving support.
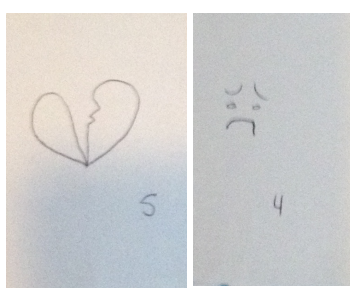

You are sad; I'm sad

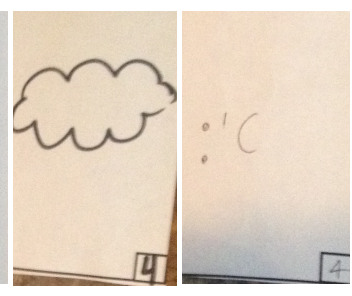

Figure 21. Sad response- symbols

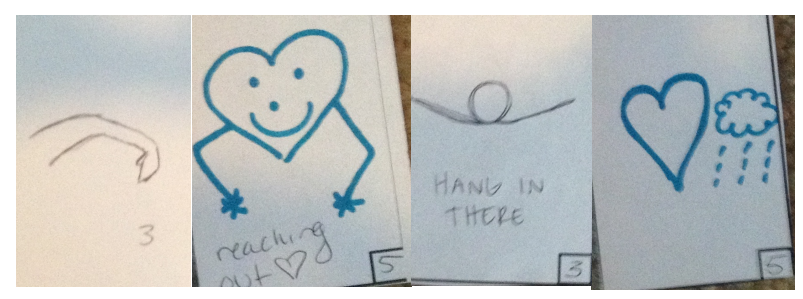

You are sad; I comfort you

Figures 22 and 23 show a summary of icons generated for ager and sad expression and different categories of response to those emotions.

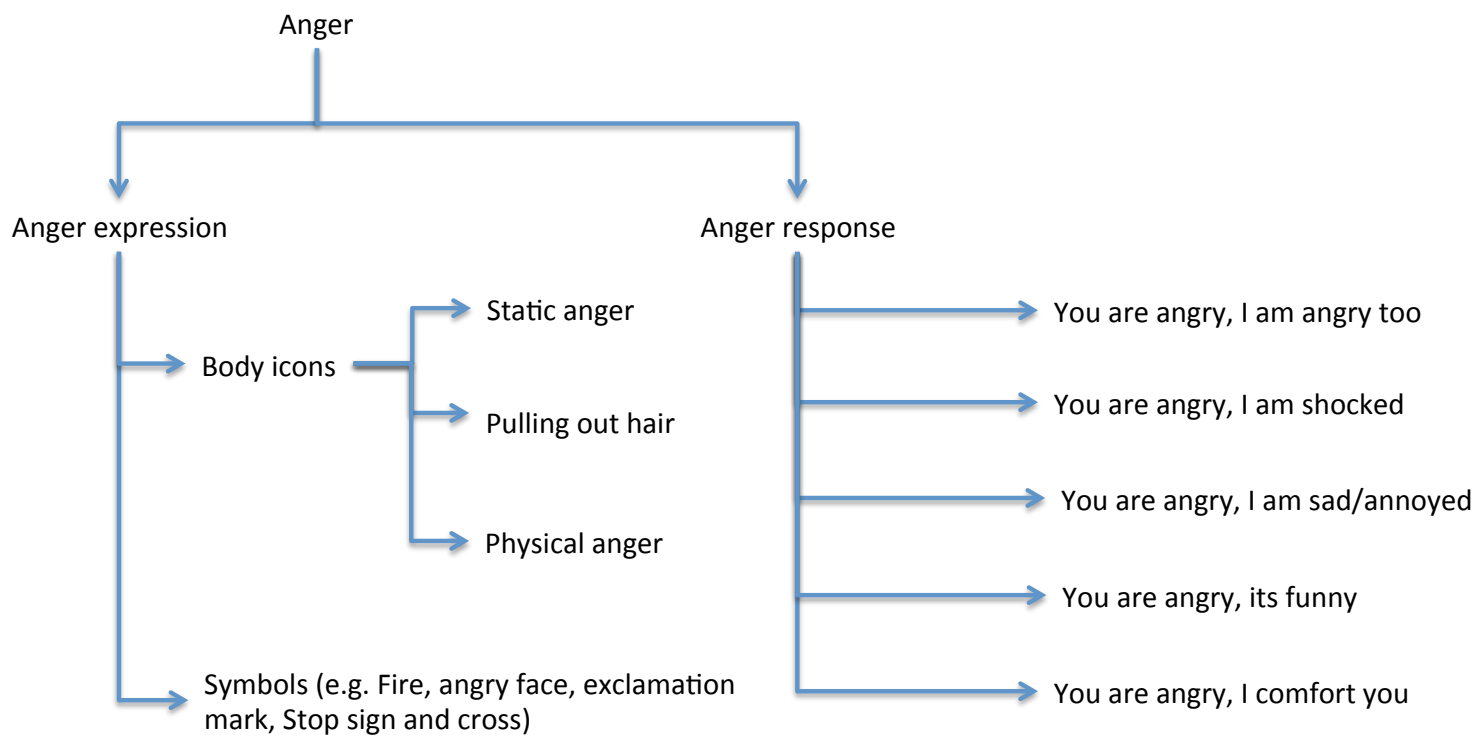

Figure 22. Anger expression and response 


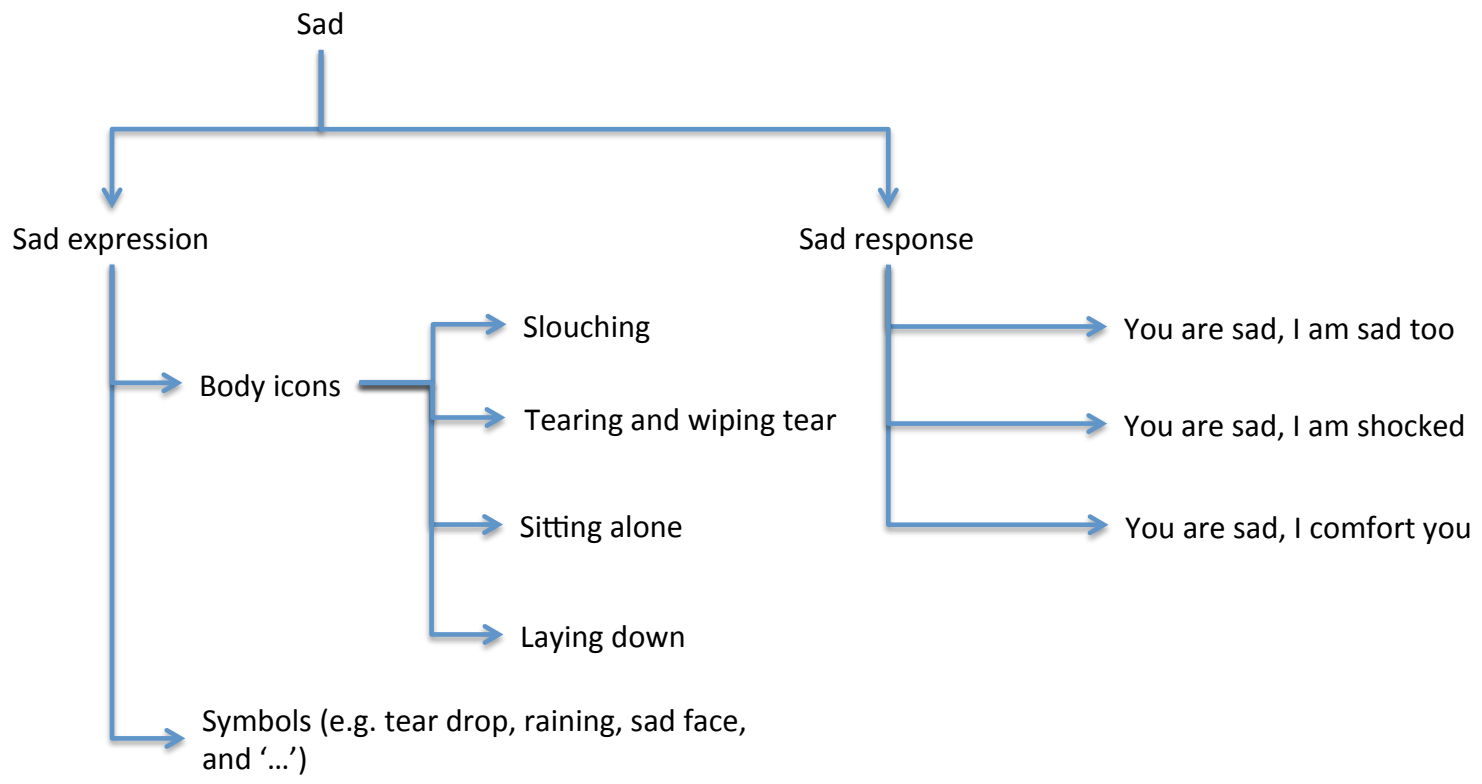

Figure 23. Sad expression and response

Another interesting theme that was identified from observing participants in the brainstorming sessions while they were creating the ideas was the process of creating multi-touch gestures. The intensity and pressure used in drawing gestures in sad and anger were different. Participants were observed to use more pressure on anger related multi-touch gestures (e.g. hard tapping), while gestures for sad expression and response were mostly generated with low pressure.

\subsection{Design solutions and prototype}

In this stage of the design process, the results of brainstorming sessions were combined with the results of conversation analysis gathered in study III to explore two main gesture-based design solutions to support emotional communication in IM.

The first multi-touch gesture based design idea was using multi-touch gestures, instead of typing or selecting emoticons to send different emotional cues users use to communicate their emotion. To communicate this design idea with users in the context 
of text messaging, a text messaging application prototype was created (Figure 24). By clicking on the icon of a gesture on the right side of the text box, a gesture board would be shown to the participant to perform a multi-touch gesture as an input and their friend on the other side would see one of the options of body icon, face icon, symbol, or word, related to that gesture.
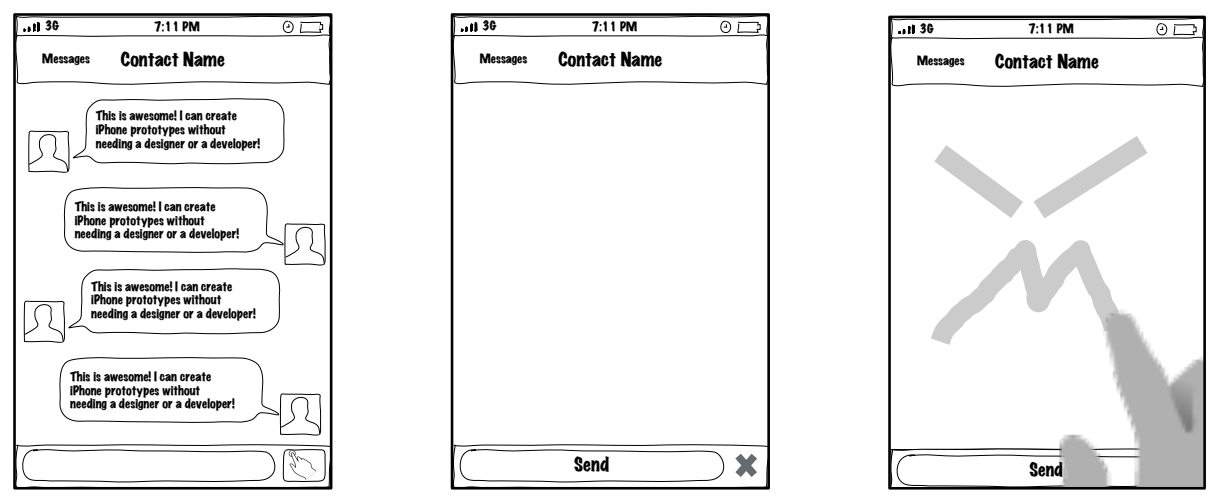

Gesture input on the sender's side
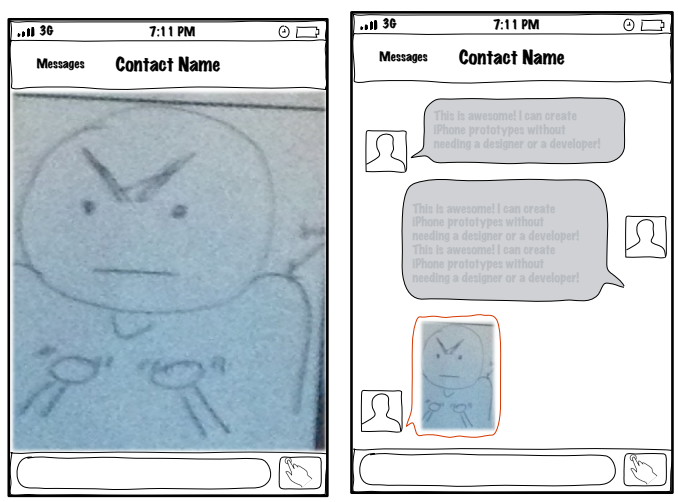

Output on the receiver's side

Figure 24. Gesture feature

Thirty emotional cues, identified from conversation analysis in study III, were selected for this stage of the design process. Emotional cues were selected from both emotional expression and response in three emotional states (happy, sad, and angry) including emotional words, lexical surrogates, emoticons, strong emotional words, and 
confirmation cues. For each cue a related gesture, body icon, and symbol were selected from the results of brainstorming sessions.

The process of matching multi-touch gestures, body icons, face icons, and symbols for every emotional cue was done by the design researcher based on the relevance and closeness of gestures and icons to specific words. Note that the design solutions (multi-touch gestures, body icons, and symbols) matched with the emotional cues were not final design solutions. They were used as triggers for participants to think about improving the solutions or create new design solutions in the following co-design sessions.

Brainstorming sessions were conducted only for sad and angry emotions because those emotions were identified as more challenging emotions to be communicated in instant messaging compared to happy emotions. The limited timing of the brainstorming sessions (two hours) also made it difficult to add the third emotion of happy to the sessions. However, the happy emotion was added in this stage of the design process, since happy is one of the main emotions that is expressed in everyday life and adding this emotion made the set of design solutions more comprehensive to cover both positive and negative emotions. Because of the time constraint, conducting another round of brainstorming sessions for happy emotions was not possible. Therefore for emotional cues that were selected from conversation analysis in a happy emotional state, design ideas including multi-touch gestures, body icons, and symbols were created by the design researcher and a design student.

Face icons were also added in this stage to explore differences between body, face, and symbols in emotional communication. Table 7 shows the list of cues and equivalent gestures, body icons, face-icons, and symbols. 
Table 7. List of emotional cues and equivalent multi-touch gestures, body icons, and symbols used in co-design sessions

\begin{tabular}{|l|l|l|l|l|}
\hline $\begin{array}{l}\text { Emotional cue } \\
\text { (Selected from } \\
\text { study III) }\end{array}$ & $\begin{array}{l}\text { Multi-touch } \\
\text { gesture } \\
\text { (Selected } \\
\text { from design } \\
\text { brainstormi } \\
\text { ng session) }\end{array}$ & $\begin{array}{l}\text { Body icon } \\
\text { (Selected from } \\
\text { design } \\
\text { brainstorming } \\
\text { session) }\end{array}$ & $\begin{array}{l}\text { Face icon } \\
\text { (Selected by } \\
\text { researcher) }\end{array}$ & $\begin{array}{l}\text { Symbol } \\
\text { (Selected from } \\
\text { design } \\
\text { brainstorming } \\
\text { session) }\end{array}$ \\
\hline Annoyed & & &
\end{tabular}




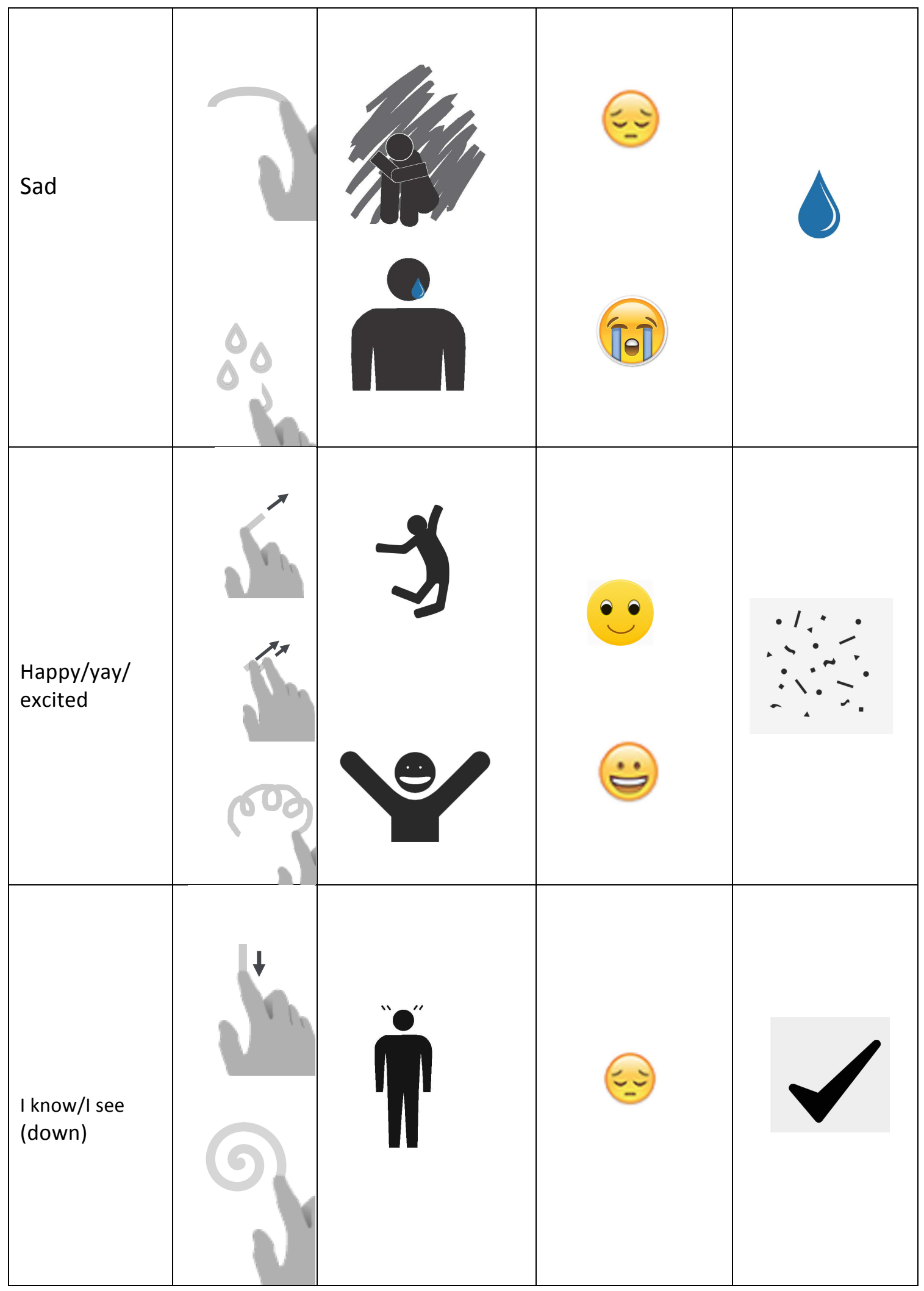




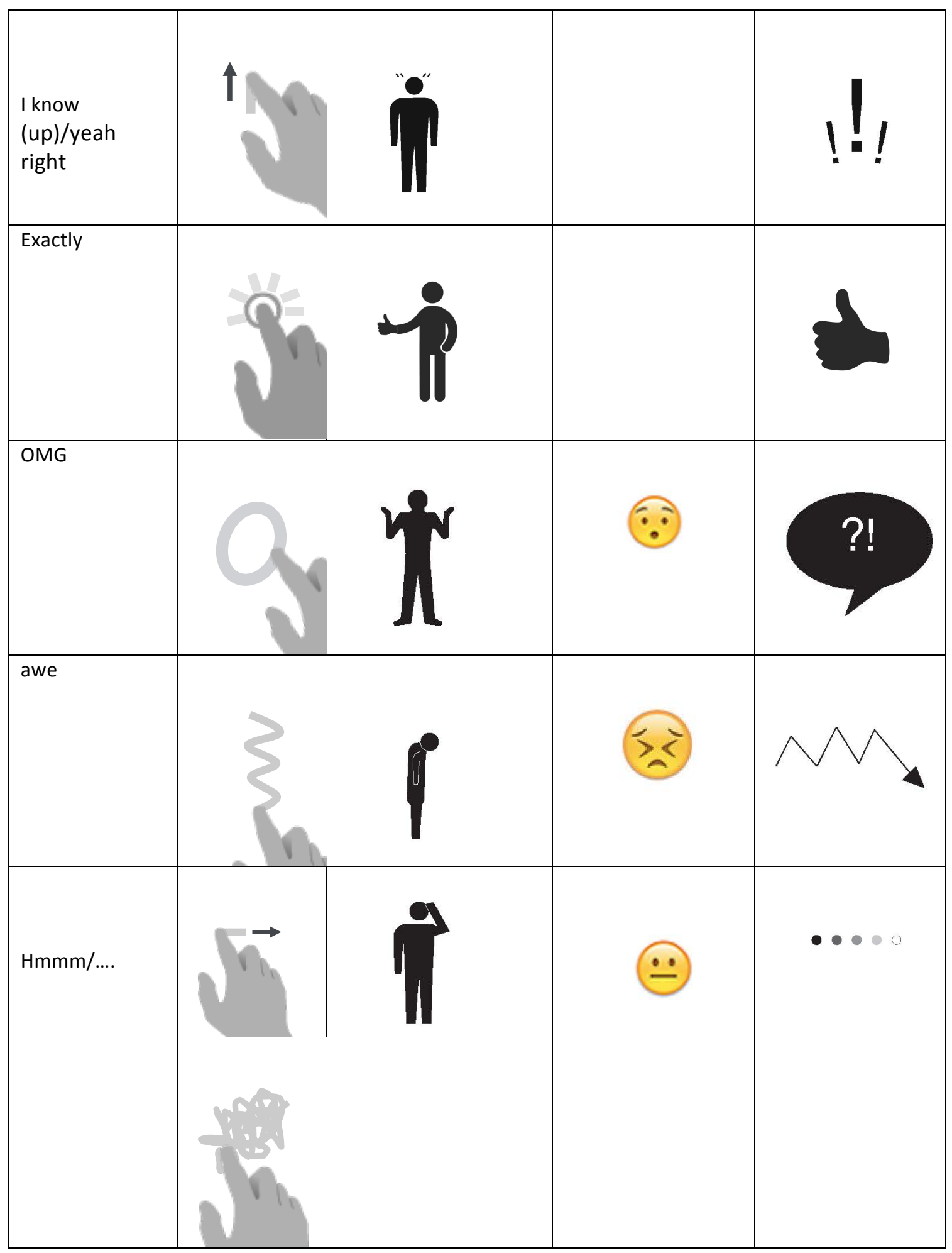




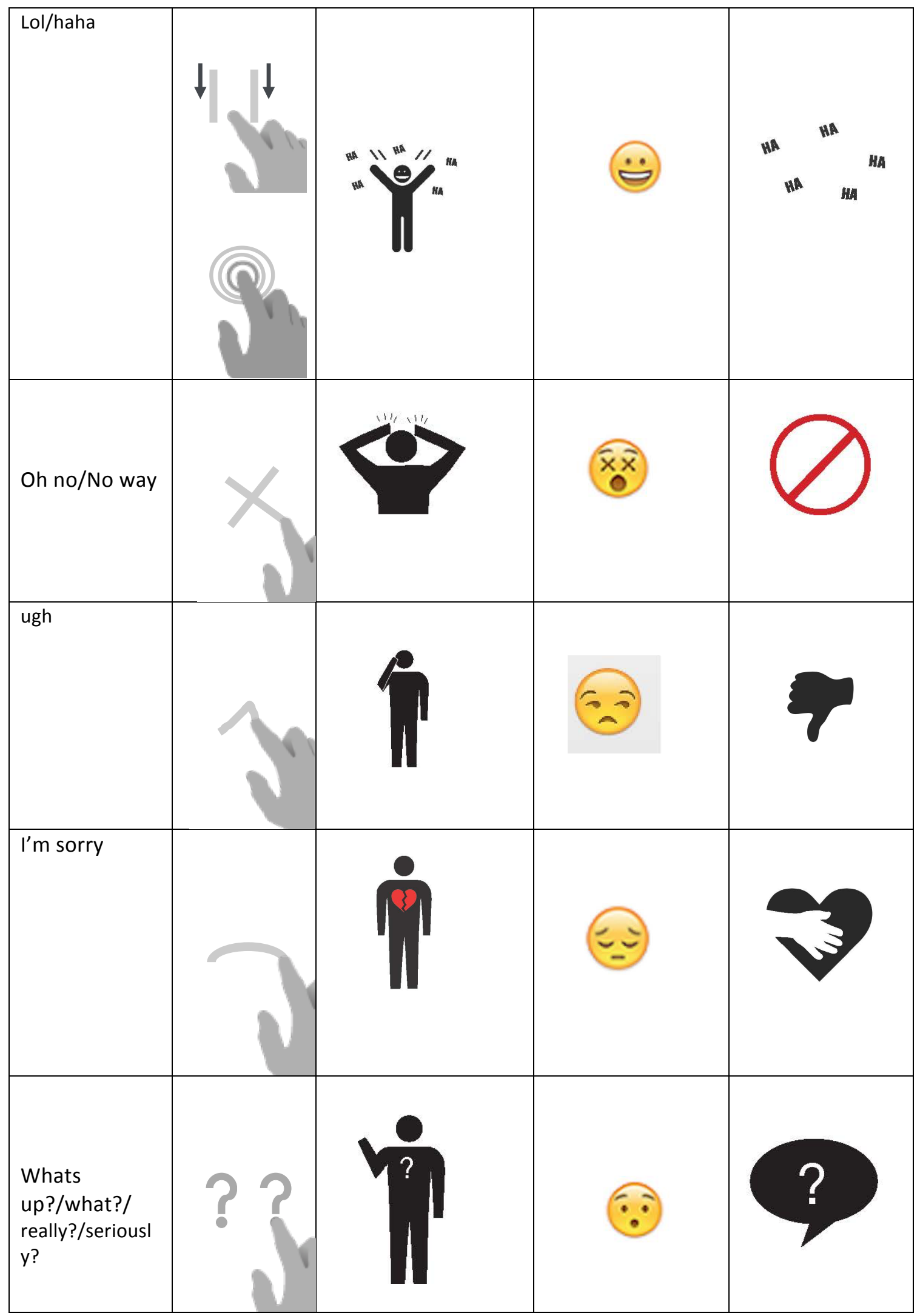




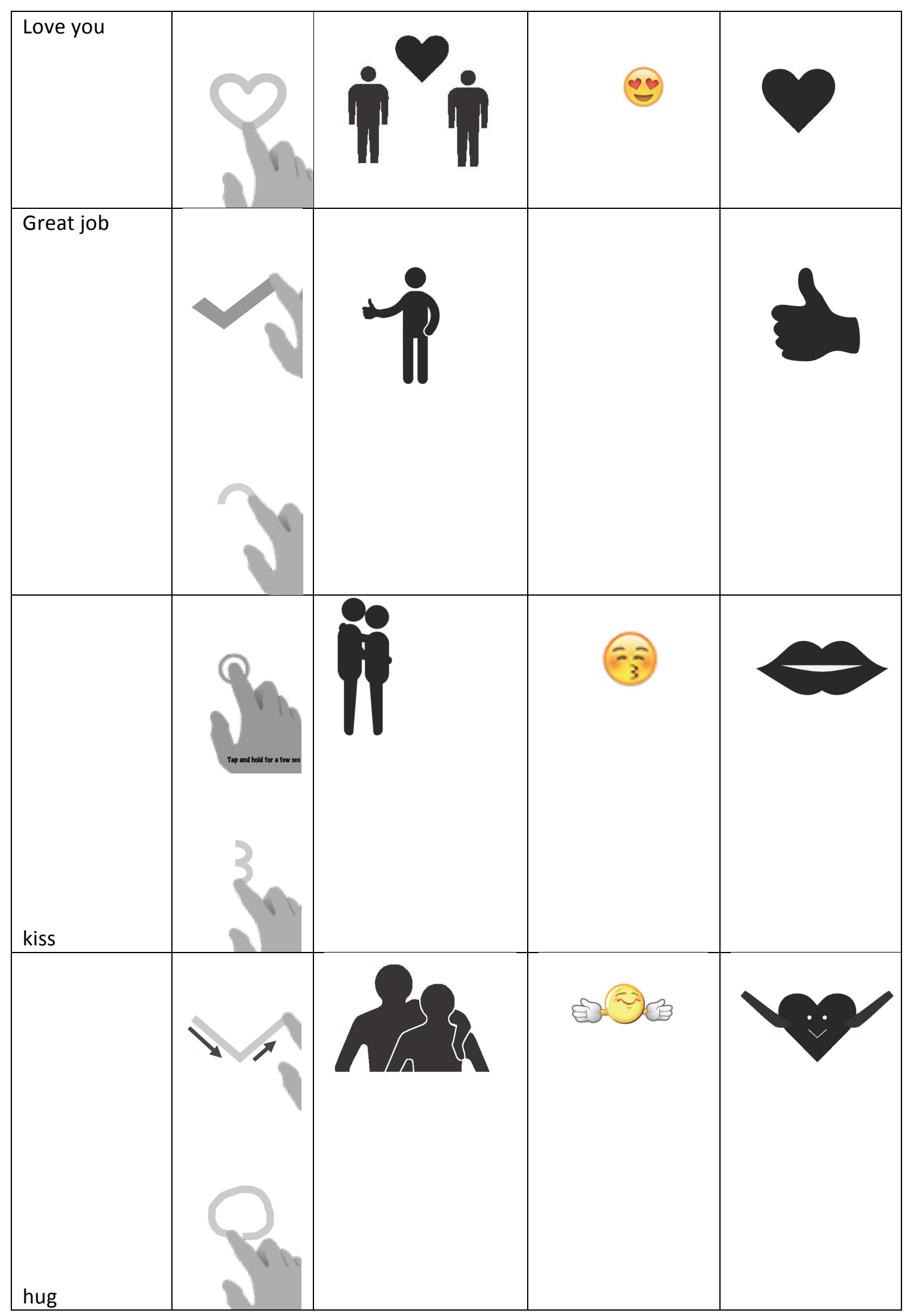


Based on the results of the conversation analysis, participants used different strategies such as using lexical surrogates or emoticons at the end of their utterances to convey the tone of their statements. The second multi-touch gesture based design solution was created to support this specific behavior with swiping text in different directions and changing the color of the text background to convey four main emotional tones of angry, sad, happy and neutral (Figure 25).

To evaluate and improve the design solutions and create new solutions, codesign sessions were conducted as the next stage in the design process.
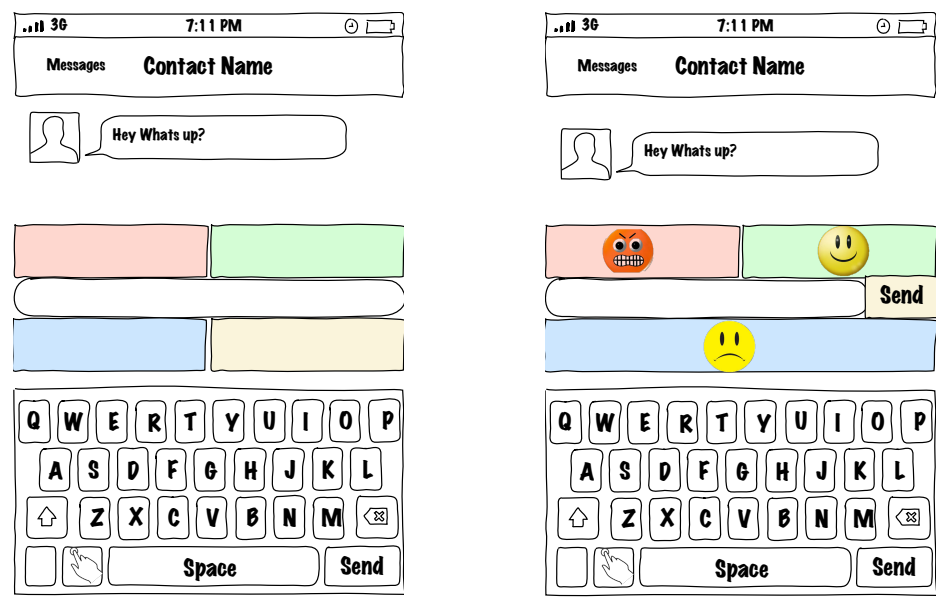

Figure 25. Swipe feature and color change

\subsection{Co-design sessions}

The main goal of the co-design sessions was to evaluate and improve two design solutions that were explained in the previous section. 30 IUPUI students in total in 15 pairs received a \$20 Amazon gift card to participate in the co-design sessions (16 in the first round, and 14 in the second round). 18 female (average age=24.6, $S D=4.3$ ) and 12 males (average age=23.2, $S D=3.2$ ) were recruited for this study. In this stage of the 
design process, careful attention was paid to recruit a roughly equal number of males and females. Eight teams were female-male, two teams were male-male, and five teams were female-female. Partners were required to be friends who knew each other for at least six months, so they were more comfortable expressing their emotions, and engaging in natural emotional conversation (Wagner and Smith, 1991; Parkinson, Fischer, and Manstead, 2005; Derks, Fischer, and Bos, 2008; Ramirez and Broneck, 2009).

After every session, the data was analyzed and minor changes in the prototype were made for the next session. However, after eight pairs, patterns on data were observed and design solutions and the problem statement were substantially refined. In the following section, two rounds of sessions and the transition from round one to two are discussed.

\subsubsection{Co-design sessions (round I)}

\subsubsection{Participants}

Sixteen IUPUI students, seven female and nine males, participated in the first round of co-design sessions. Two teams were female-female, three were male-male, and three were female-male.

\subsubsection{Procedure}

Participants were asked to fill out a consent form and demographic questionnaire upon arrival. Then they were asked to sit on each side of a table. The researcher was sitting in the middle to facilitate the session. Two video cameras captured the two sides of the table to record participants' hands and interaction with the cellphone as well as participants' spoken comments and think aloud data. Figure 26 shows the setting of the experiment. Participants were given paper prototype cellphones in addition to a conversation that included all the words shown in table 7. Emotional cues were in bold during the conversation. Participants were asked to role-play through the conversation 
line by line and as they got to the bold cues invent a gesture on the screen of the phone prototype to express that cue. They were asked to think aloud while doing so. After they created a gesture, the gesture identified by the designer in table 7 was shown to them and they were asked to compare that with the gesture they created.
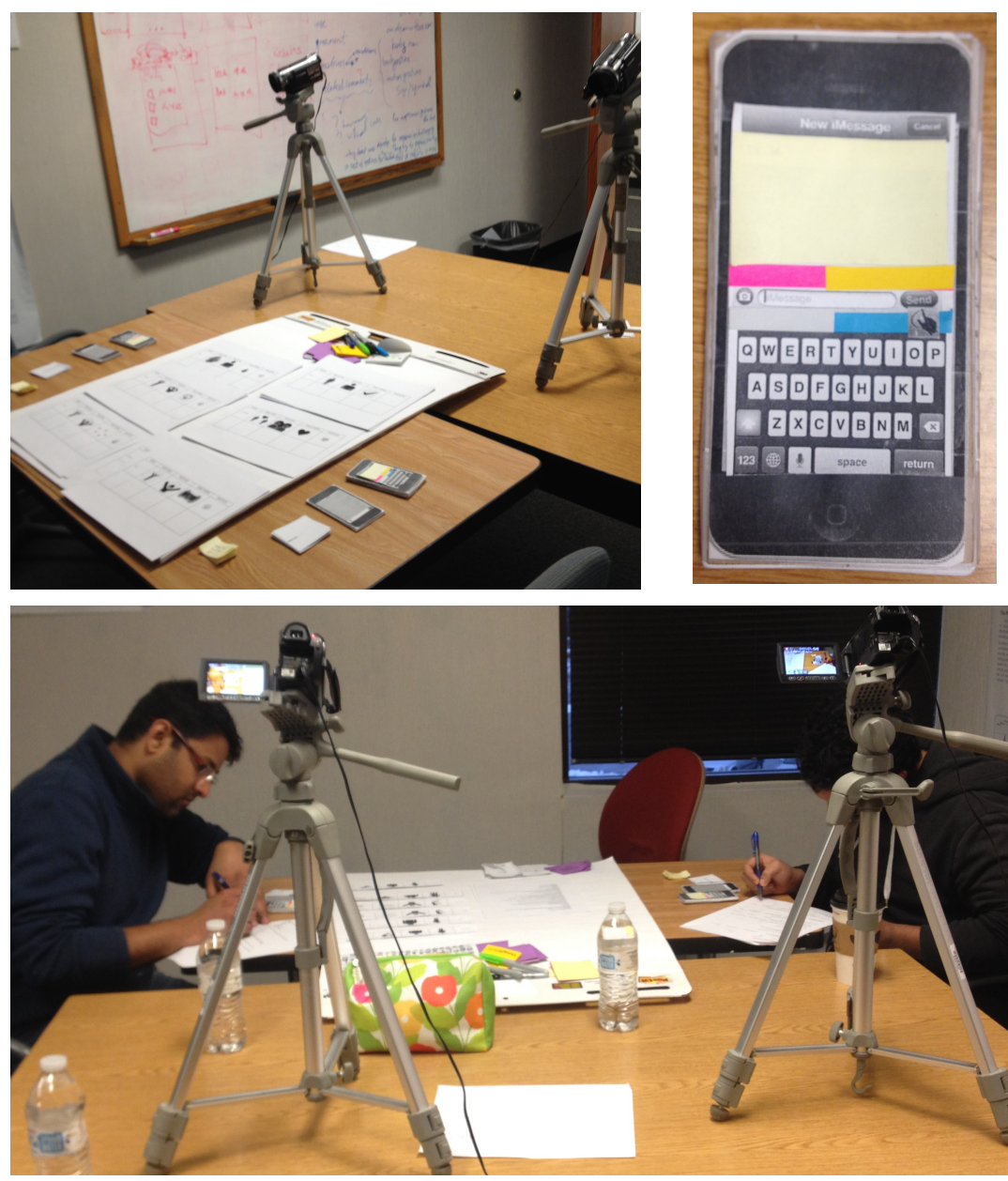

Figure 26. Co-design session round one setting

Participants were also shown the related body icon, face icon, symbol equivalent, and word to each multi-touch gesture on table 7 and asked which option they preferred their friend on the other side of the communication receive after they enter that specific gesture. Participants were also told that they had two options of using the gesture or 
swiping the text in four directions to convey their emotion. They were free to pick one or the other throughout the conversation.

\subsubsection{Results}

Video data from the first eight pairs of participants was analyzed and the following main themes related to the design solutions were identified:

- Gesture board: moving from one screen to the other for drawing seems cumbersome to users. "For gesture I prefer to do that on the text screen. Moving to another screen of the gesture board is too much", "Going to another screen is not good. I want to know who I'm texting and what are the previous texts".

- Being in control and avoiding surprise: Desiring to be in control was another main theme identified from data analysis. Participants wanted to be able to check exactly what the application would send to their friends before hitting the send button. It would make them able to change the unwanted state before sending the text. This concern was reported by participants for both color and gesture design features. They reported they liked to know what color their text is before sending and also what emoticon would be sent after doing a gesture. "Its easier if it shows me what it is going to send so that I know before sending it", "I want to know what will be sent when I do the gesture", "change the keyboard color so that I know the text is red because I could see myself putting red by mistake and sending something. But if you change the keyboard I know that I'm typing in red".

- Options on the receiver side: What participants (body icons, face icons, words, or symbols) liked to see after entering a gesture varied by participants and their emotion, which was explored more in the second 
round of design sessions. However, participants reported using gestures to retrieve one specific emoticon or word is not time efficient and it was sometimes easier and faster for them to just type the word or select the emoticon instead of using the gesture for brining up the word/emoticon. Giving more than one option related to the multi-touch gesture was suggested by participants as well. In that case, they would be able to choose what they would like to send among a selection of related emoticons and words.

- Overwhelming number of gestures: There were too many gestures and it was hard for participants to remember all of them. Participants started to put similar words in the same category of the gestures and created ten main categories that all the words could fall under (Figure 27). "I think some words are the same for example what? and Whats up? Or ummm... and sigh are the same. Sounds good, agree, good job, I know, and yeah are the same too".

Several other themes were also identified, which needed further investigation in the second round of the design sessions. For example, when and why participant's preferred body icons, versus face icons, versus symbols or words. Therefore the next round of co-design sessions was conducted after changing the prototype based on the results of data analysis, which is explained in the following section. 


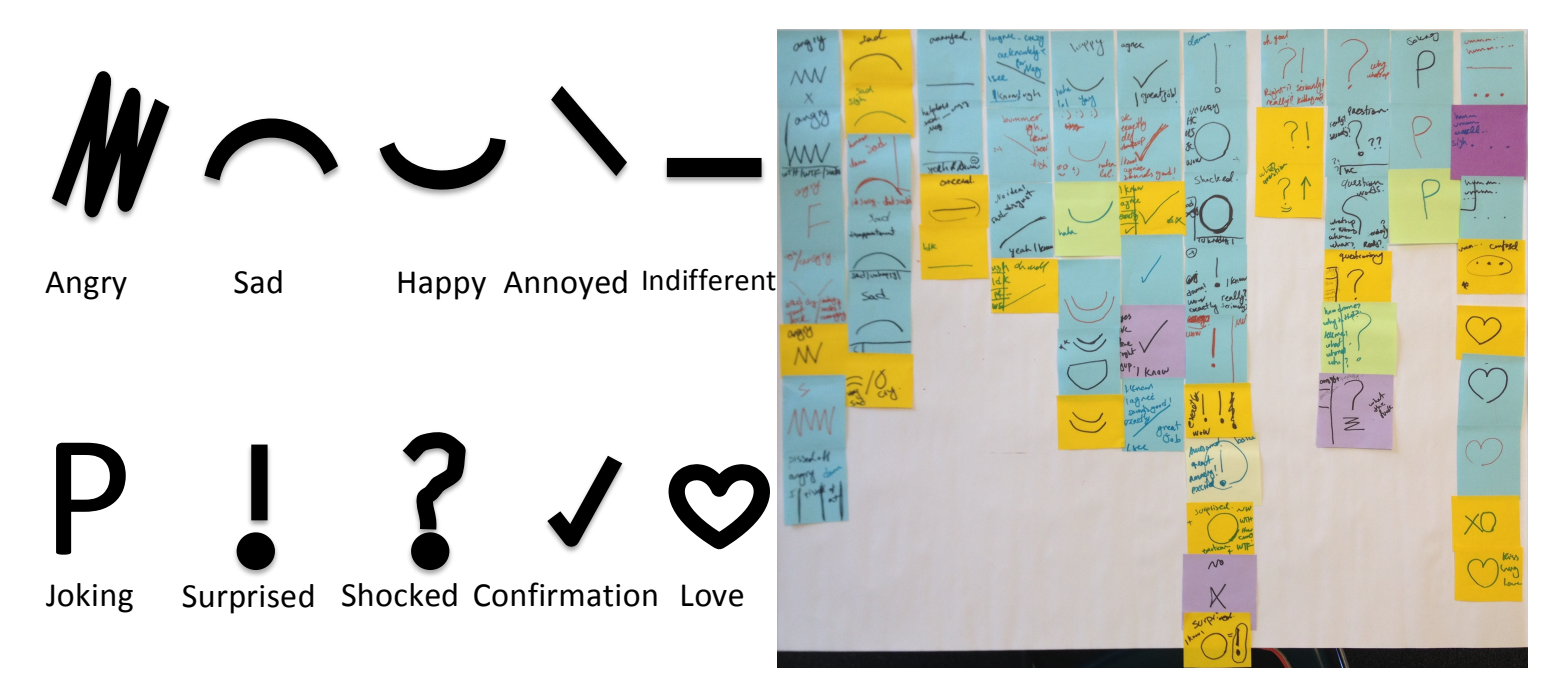

Figure 27. Multi-touch gesture categories created in co-design session round one

\subsubsection{Design Solutions and prototype refinement}

Based on the results of the first round, the following changes made on the design solutions:

- Since color swiping did not give the users the feeling of control on the color before sending the text, it was changed to the three buttons below the text box. As the user clicks any of the colors, the keyboard and the background of the text-box color would change to notify users of the color change. Figure 28 shows the refined color feature.

- Since moving to a new screen for a gesture board was time-consuming, and also users lost track of texting, the gesture board was moved to the space above the keyboard. Users needed to double click on the screen and the gesture board appeared. Figure 29 presents the refined gesture board. 

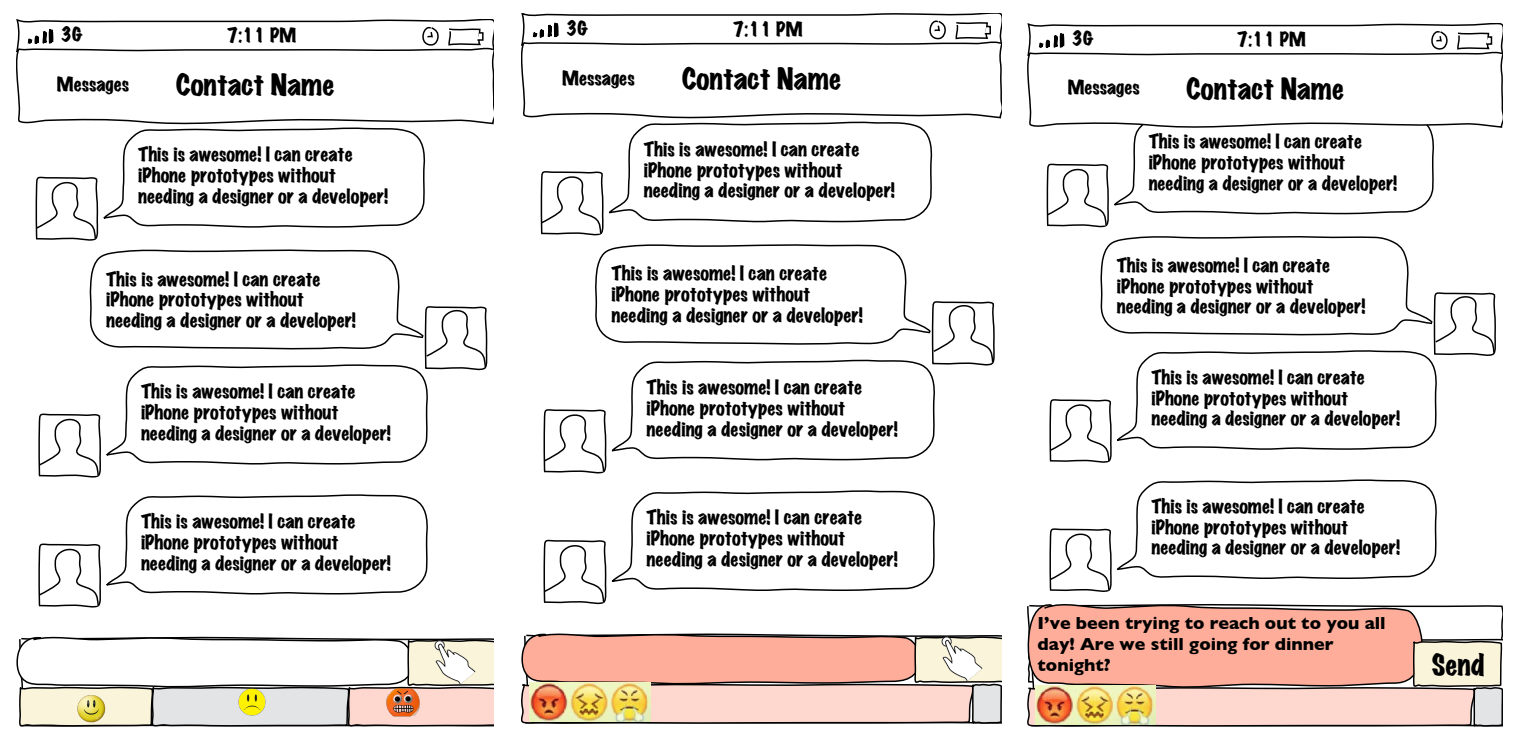

Figure 28. Refined color feature

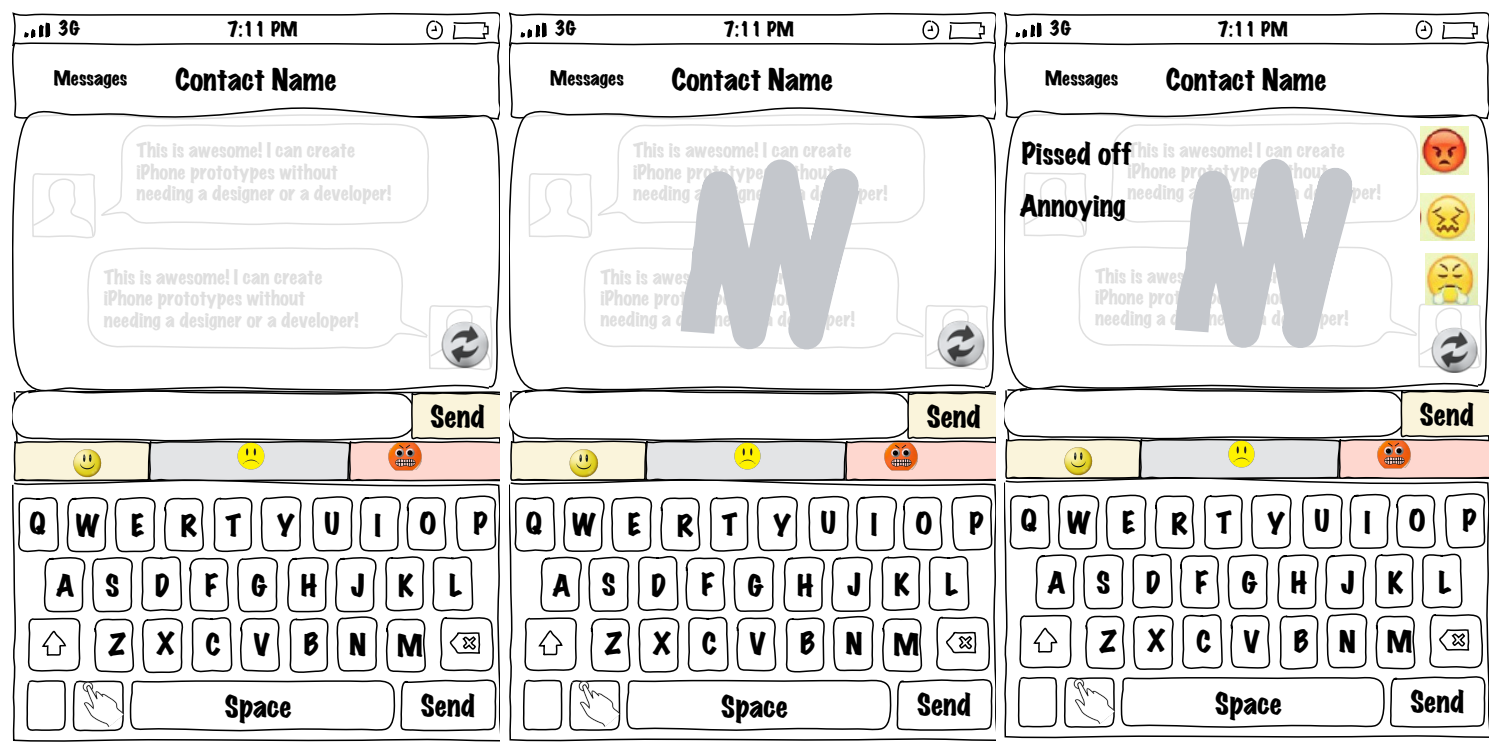

Figure 29. Refined gesture feature 
- Since participants categorized all the words under ten different gestures, the gesture feature changed in a way that as the user input the gesture on the gesture board, a list of all the options of body icons, face icons, words, and symbols related to that gesture would be shown and they could select one or more options to add to the text box (Figure 28).

The prototype was redesigned based on the above changes and the second round of co-design sessions was conducted with the revised design ideas.

\subsubsection{Co-design sessions (round II)}

The main goal of this second round of co-design sessions was evaluating and improving ten categories of gestures (Figure 27) and identifying different face icons, body icons, symbols, and words that go under each category. It also focused on improving the redesigned color feature.

\subsubsection{Participants}

Fourteen IUPUI students, eleven females and three males participated in this study. Four teams were female-female, and three teams were female-male.

\subsubsection{Procedure}

Participants were asked to fill out a consent form and demographic questionnaire upon arrival. Then they were asked to sit next to each other at a table. The researcher was sitting on the other side of the table to facilitate the session. One video camera captured the table and participants to record participants' hands and interaction with the cellphone as well as participants' spoken comments and think aloud data. Figure 30 shows the setting of the experiment.

To start the session, the main design features were explained to participants. Then they were shown all the different gesture categories that were identified from round I with some examples of face icons, body icons, words, and symbols that were identified from round I and brainstorming sessions. They were asked to give their feedback on 
different gestures including what category of emoticons and words each gesture referred to. They were also asked to make their own set of related face-icons, body-icons, symbols, or words for each gesture category. Finally, they were asked to imagine they were in different emotional states of sad, angry, and happy and would like to text each other about it. They were asked to role-play and text with each other using the main features of gesture and color.
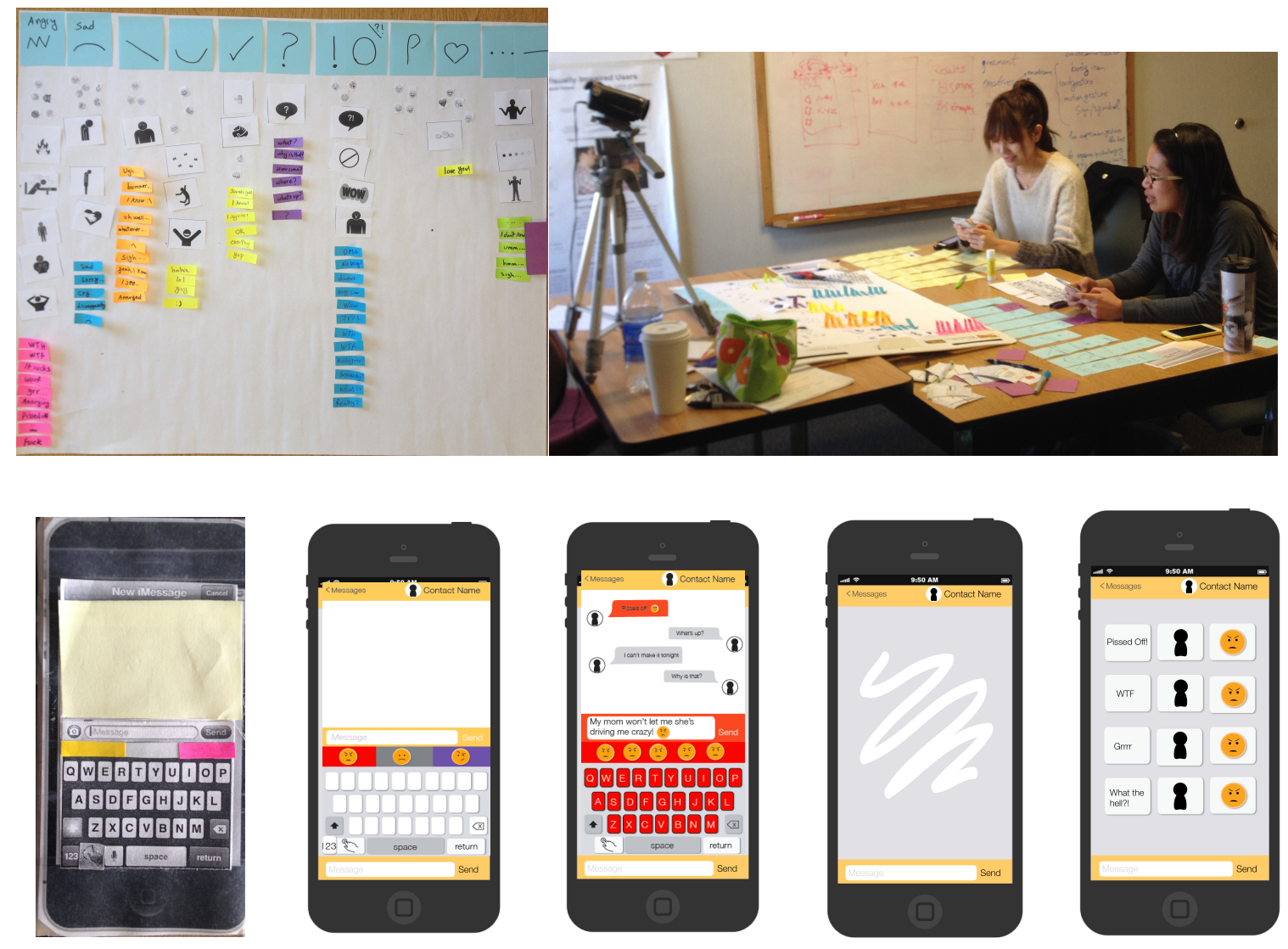

Figure 30 . Refined gesture feature 


\subsubsection{Results}

Emoticons and words that participants put under each category were combined as figure 31,32 , and 33 show.

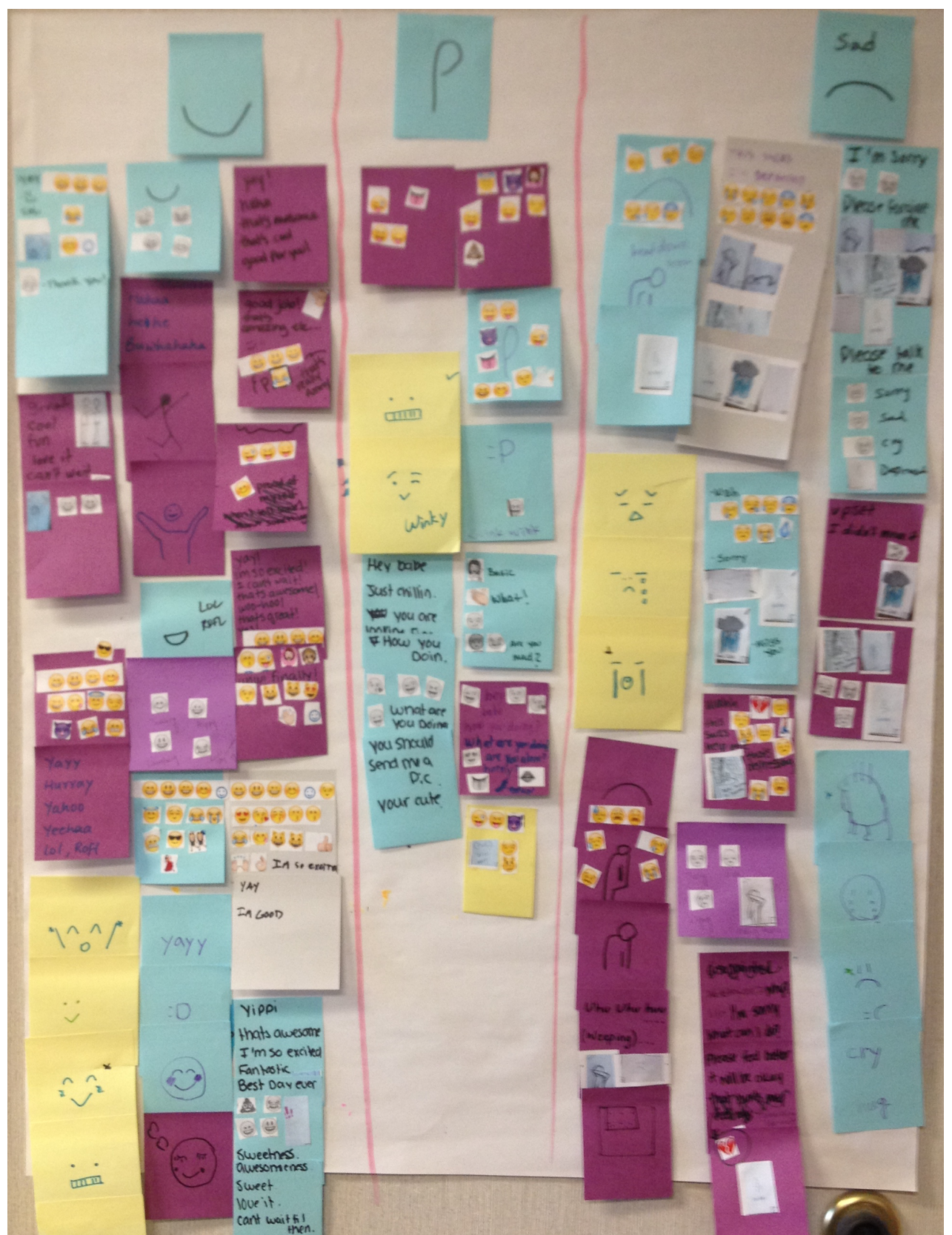

Figure 31. Gestures categories (happy, joking, sad) and sets of body icons, face icons, symbols, and words related to each category 


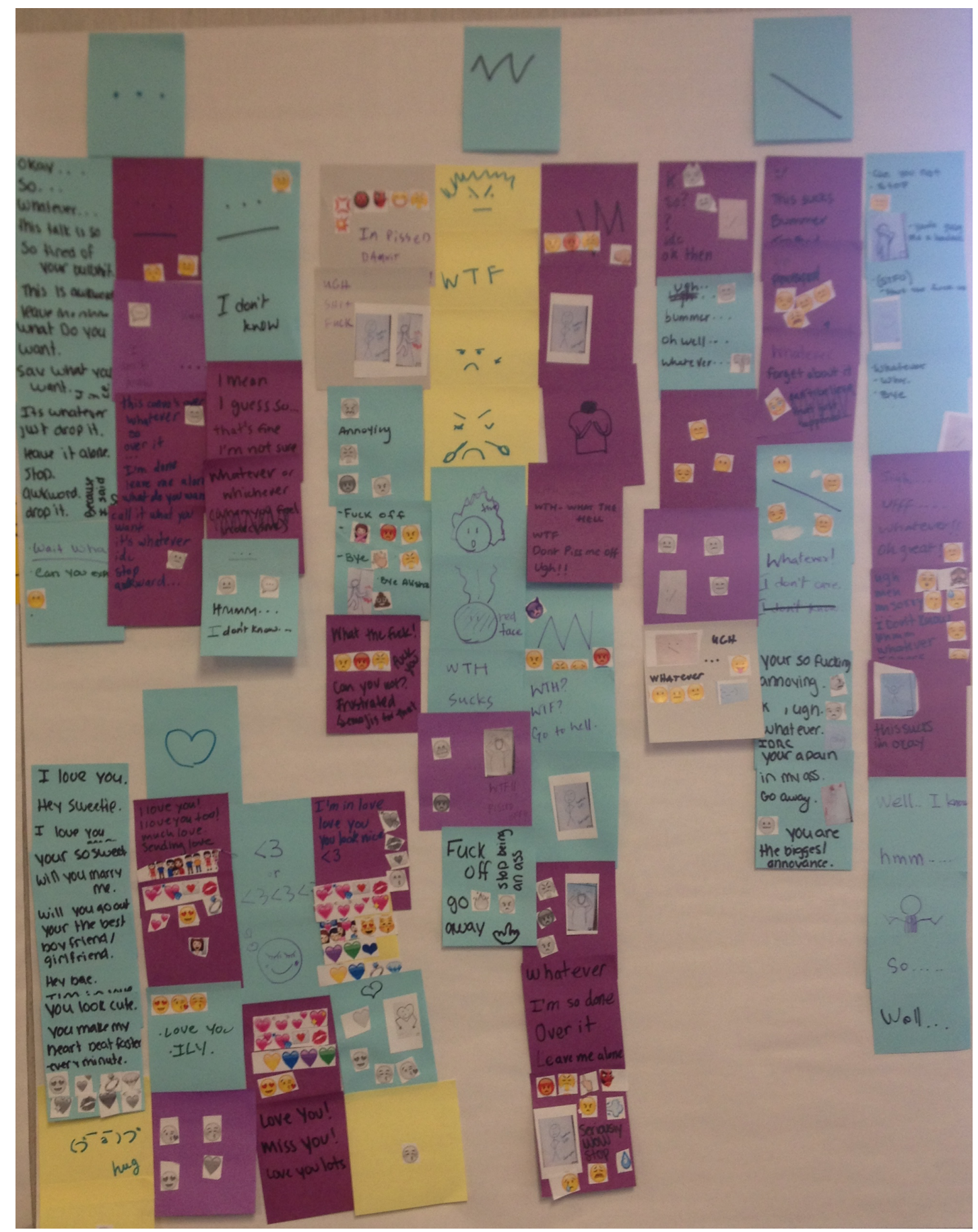

Figure 32. Gestures categories (indifferent, angry, annoyed, love) and sets of body icons, face icons, symbols, and words related to each category 


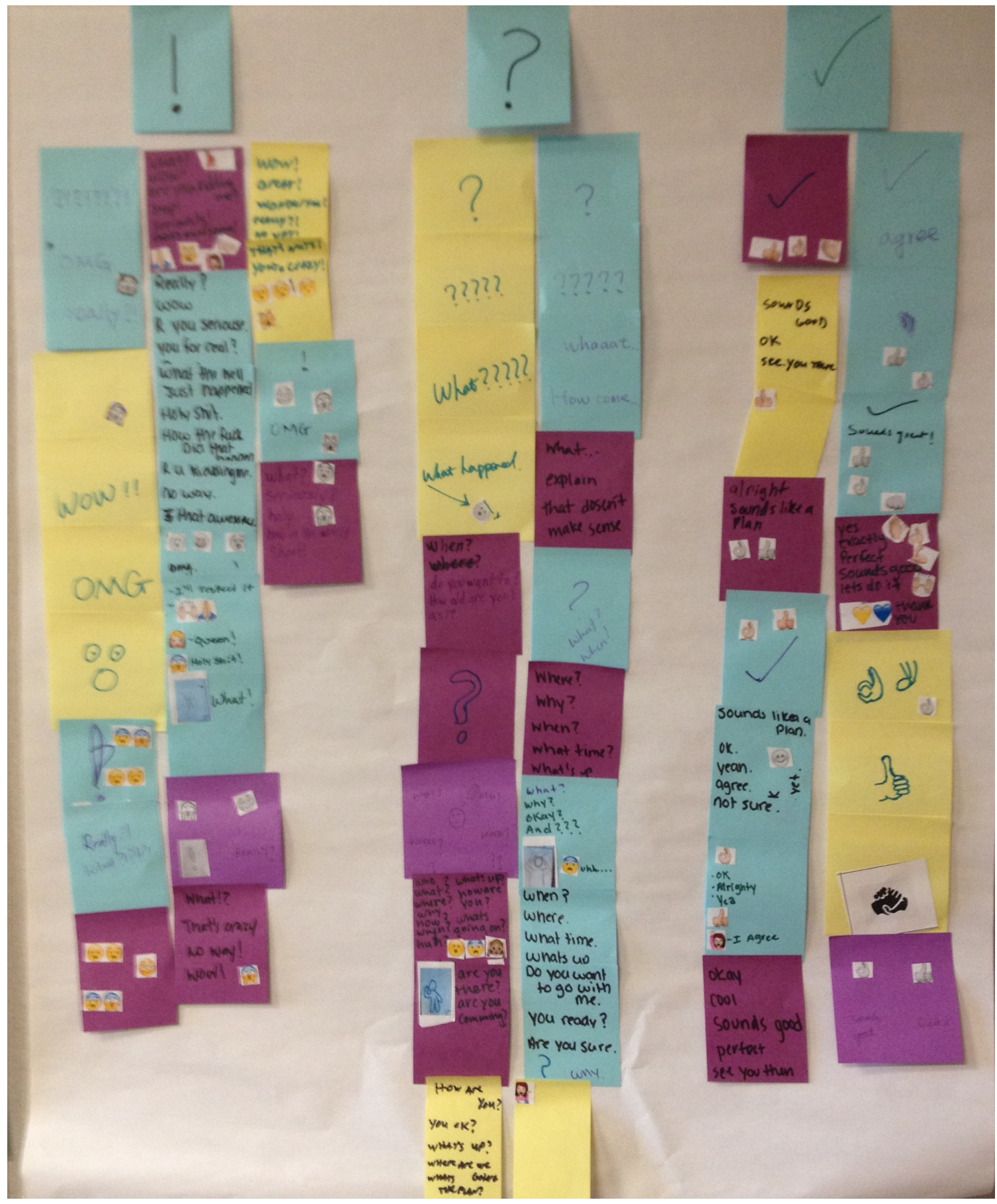

Figure 33. Gestures categories (surprised/shocked, questions, confirmation) and sets of body icons, face icons, symbols, and words related to each category 
Video data from brainstorming session round III was analyzed and the following themes were identified through theme analysis:

Why text messaging?

One of the themes identified in data analysis was the text messaging usage in everyday life. Participants reported different reasons why they use text messaging in everyday life and what technology they would like to use for text messaging. Time was identified as an important factor in mobile text messaging. Quick communication with the least effort was the main reason participants reported for the usage of the mobile text messaging. For example, "I use texting for quick communication", "If it can help me to send faster texts I would use it. If it slows me down I wouldn't use it".

They also reported they use text messaging and specifically emoticons to stay in touch with people that they already know and have an emotional connection. For example, "if someone texts me we have that close relationship that allows me to send emoticons...", "I use emoticons when texting people I know".

\section{Emotional expression styles}

Regarding emotional expression, IM users were divided into two categories. The first category included users who were not willing to express their emotion regardless of the medium. Willingness to express emotion varied among different participants and was different among the three emotions of happy, sad, and angry. Some participants reported that mostly for negative emotions, specifically anger, they do not express their emotion. Motivating this group of users to express their emotion using gestures and color design solutions was challenging, since the reasons behind their behavior were mostly related to different personality traits or cultural background. The following are some of the comments reported by participants: "I don't get really angry", "When I'm sad or angry I don't talk that much", "when I was young I used to get mad and release it but as I got older I learned I should think about my anger first before expressing it". 
The second group included users who were willing to express their emotion in text messaging. Users in this group were divided into two subcategories: first, those who were already satisfied with the current text messaging with regard to emotional communication, and second, those who reported the current text messaging did not completely support them in emotional expression and they sometime prefer to use other media such as phone or face-to-face communication to convey their emotions. For example, "If I am actually sad, I would be calling not texting...its hard to express your real sadness in text", "I think I'm pretty good in expressing my emotion to my friends in texting".

The second group of users (who were willing to express their emotion in text messaging) reported the most positive feedback on two main design features that were presented in the second round of design sessions. Therefore, special effort was made to understand how this group of users communicated their emotion and explored hidden and obvious challenges they had with emotional expression in text messaging to improve the design features.

Emoticons in emotional communication

Using current text messaging applications such as What's App, Wechat, Facebook messenger, or the iPhone text messaging application, participants reported different usage of emoticons to communicate their emotions:

- Conveying the tone of the message at the end of the text or giving emotional feedback upon receiving a text. For example, "I use emoticons at the beginning of the statement to give feedback first and then text and then put another emoticon at the end to show the tone of my text", "I usually use emoticons after text." 
- Making their message more genuine. For example, "when I use emoticons it makes the conversation more genuine and shows that I mean something."

- Emphasizing on emotion and making sure the person on the other side of the communication understands their emotion. For example: "I just add emoticons to make sure he understands my emotion."

- Showing extreme emotions. For example, "emoticons for me are used in extreme. When l'm very mad or excited."

- Replacing words with emoticons. Some participants reported it was easier for them to send an emoticon to convey a message rather than using words. For example, "Sometimes emoticons convey the message better. Showing the face of surprise is better than describing it in words."

Despite the usage of emoticons, participants reported some challenges they encountered in using the emoticons. They reported that there are too many emoticons and it makes it harder for them to find the emoticon they need among all the options. Some participants also reported emoticons are out of reach and it makes them not use them. For example, "Whats app has too many unnecessary emoticons that no one uses and searching is cumbersome", "I don't have the patience to search for emoticons....", "I don't use emoticons, emoticons are out of reach and that's maybe why I didn't get to use them".

The following sections discuss the usage of different types of emoticons including face-icons, body icons, and symbols more in detail and compared with the usage of words in emotional communication. 


\section{Face-icons}

Face-icons presented in the design sessions were selected from the list of currently available emoticons on the iPhone text messaging platform. Participants reported different reasons behind using face-icons such as being the most common available option in current applications, importance of communicating facial expression, and the proper alignment of face-icons with the text. For example, "Sadness by face is easier to understand", "When I'm texting I like to use emoticons because they align with text", "I think face gives all the information you need."

The usage of face-icons varied among participants and different emotions. Some participants did not use face-icons to express their negative emotions, especially anger, since they were cartoony/silly/funny and the other person would not take them seriously. For example, "when I feel sad I don't use face icons or when I am mad. I don't think the other person takes me seriously if I use them." "When I am in fun/good mood I use faceicons but not when I am in a bad mood. In a bad mood I just want to explain in words that I'm mad", "I use mad face icons when for example my professor gives a lot of homework, to show that I'm angry. But it's more like funny angry", "face-icons in sad and angry are funny“, “I don't use colorful icons when l'm sad or angry because it's not real and shows that I'm already over what made me angry ".

Although some participants reported no usage of sad and angry face icons, some participants reported they use them even in sadness and anger. For example, "I use more when I am sad and angry (its easier to convey anger and depression) especially angry face icons", "sadness is a good emotion to show and it shows affection to the other person and its genuine... In sad you want to show the other person that you are sad and you miss them or you are sorry. There is more emotion toward it when you use face icons", "I use face-icons of angry when I am upset. I don't see that as cartoony" 
Body icons

Participants reported some advantages and disadvantages of body icons. They liked body icons because they conveyed emotions better than face-icons, especially for some extreme emotions such as very sad or angry. For example, "I would personally use the body icons because I think they are more expressive", "If I am really angry I prefer body expression that shows I'm really angry", "Body icons sometime get the point across better. Emoticons are generic, but the body icons could display the emotion better than emoticons. Like when I was defeated or gave up I would use slouching body",

Participants also reported they would use body icons that were presented to them because they had more character than face-icons. For example "I like body icons they have more humanized feeling. They have more character and meaning". Especially for participants who did not use face icons because they were funny/cartoony, body icons were more interesting because they were simple and less colorful. For example, "I use body icon to show that I'm really angry because it's so simple and not funny", "Sometimes emoticons are too much, l'd like how simple body icons are".

Finally, some participants reported they prefer body icons because they were new and they liked to explore new options. For example, "I like body icons because they are new, and I like exploring new things", "I prefer body icon for angry. It shows the intensity of the emotion and also face icons become so common, but body icons are new and fun to use".

Body icons requiring more space than emoticons was reported as one of the disadvantages of using this type of icons. Participants also reported some body icons were not clear what they were trying to convey. For example, "Depending on the situation, I would use face icon or body icon. If they take too much space I prefer emoticons because they align with my text", "Some body icons are not clear." "body icons sometimes are hard to understand." 


\section{Symbols}

Some participants preferred symbols to express their emotion since they represented an action and not a cartoony face/body. However, some participants reported it was hard to get the meaning of symbols since they were abstract. For example, "I don't use symbols because I don't get them. For example "why do you send me fire or rain? I am more literal and logical", "I prefer rain and tear as sad. I take stuff as double meanings so I like the abstract and symbols", "the symbol is the best because it's an action it's not a silly person", "If I get fire from him, I won't get that he is angry"

Words

Participants reported that words, rather than emoticons, were sometimes a better way to convey emotions, especially for negative emotional expression and acknowledgment. For example, "for angry you curse so much and don't need emoticons". "for the sad response I like to send words not emoticons or symbols", "I prefer to send the word instead of emoticons. I don't want the other person to think I put no effort in texting specially when she is sad or angry." "Symbols are just too easy, and the other person might think that I didn't put any effort to empathize with her", "For acknowledgment I prefer to use words to show the other person that I care and spent time to answer you".

Using words and not emoticons in text messaging was reported as a sign of sadness and anger by some participants. For example, "I don't use as many emoticons when I'm sad or angry. I feel like when I don't use emoticons it shows the sadness and anger by itself. Usually, when you don't use the emoticons, it shows that you are not happy."

Participants also reported that for some words there were no related emoticons. For example for "No way" or "I know" there is no emoticon that could be replaced. "Sometime I prefer words over emoticons. For example for 'no way', I rarely use 
emoticons. I prefer words". They, however, reported that they would use emoticons in combination with words to convey the emotion of some word. For example, when they use "I know :)" for a happy response and "I know :)" for a sad acknowledgment.

Other media

Finally, there was a group of participants who were willing to communicate their emotion via text messaging, but they reported that none of the above channels of emoticons and words in text messaging are enough to comprehensively support emotional communication. Therefore, they have to use other media sometimes to communicate their emotions. This was reported mainly for negative emotions such as sadness and anger.

Gesture-based design solution

Participants expressed their interest in using gestures as a unique feature of the application that made it easier for them to find related emoticons to their emotion and eventually accelerated their texting. "Although it might take some time to learn how to interact, it will eventually speed up texting", "Instant messaging is about communicating fast and this application does that. Like the drawing...", "I really like the gesture on the screen and that's the main feature that differentiates this application from other applications in the market."

Participants reported that the gesture feature supported them to overcome the challenge of spending time going through a long list of emoticons to find the specific emoticon they need to send. "I just need it to tell me what to do. With all the emoticons we have I probably use five of them. If you give me just a few for the exact emotion that I have, I would choose one instead of going through all of them", "I like the gestures. Now I have a problem searching emoticons. But gestures made it pretty easy", "there are a lot of emoticons that I don't understand them and don't use. There are a lot of emoticons that I need to search among them. Gestures help to find what I exactly need." 
Another theme that was identified was gesturing and supporting expressivity. Some participants reported that they felt more expressive when they draw a symbol to find a specific emoticons. However, it varied for different emotions and the amount of time participants were willing to spend for texting. "I think it does connect me to my emotion more.... For example, specific to the anger emotion, I could see myself when I draw it I express my anger too", "When I feel happy and sad I do the gestures but not for anything else", "Depends on the situation if you have time you enjoy and like to draw but if not you just draw to get the emoticons", "Drawing the anger made me release my anger", "Just visually drawing make me feel more expressive."

Participants also reported some other challenges that this feature has the potential to help IM users to overcome. "My mom can't type, sometimes she presses two letters at one time. She could do the gestures. Its great for people who can't type". "I think this application would be very helpful for people who have difficulties expressing their emotion. For example, autistic children."

Despite extensive positive feedback that was received for the gesture feature, there were some limitations with this design solution. Participants reported that it would be a learning process to learn and get used to this feature of the application, since they were used to the emoticons button on the keyboard in current applications. It also takes some time to learn different gesture categories. Comments on the learning process emphasized the importance of creating a tutorial to introduce this feature to new users.

Using gestures was specifically very interesting to the group of users who were willing to communicate their emotions. For users who were already satisfied with the emoticons and current text messaging application, the gesture feature was reported as a great way to quickly reach the related emoticons and words they wanted to use.

The new list of emoticons such as body icons and symbols were specifically interesting to users who were not satisfied with the current emoticons because they were 
funny or degraded their emotions, especially in sadness and anger. Presenting both emotional words and emoticons was another interesting point reported by participants that encouraged them to use both words and emoticons to clearly communicate their emotions. Using gestures as quick shortcuts to some common words was also interesting for users who mainly used words in communicating their emotions. Although sometimes it was easier for participants just to type a word rather that doing a gesture to get that option, especially when the word was combined with other words in a sentence, for example: "damn it was tough" or "Awe that's so cute!".

In the second round of design, ten gestures categories (Figure 26) were also evaluated and the following are some of the main themes that were identified based on the users feedback:

- Some gesture categories overlapped. There were some emoticons and words that participants used in more than one category. "happy overlaps with love", "Awe could come under happy and sad", "I would put the tongue out [emoticon] in both 1 and $P$ categories", "You could be sarcastic [tongue emoticon] when you are in different emotions. For example; when I am sad or angry, I use tongue [emoticon] as more I'm bitchy. But when I am happy I use it to joke around“.

- For emoticons that were used in more than one gesture category (e.g. tongue out, hugging, shocked), participants used the combination of that emoticon with the emotion face-icon. For example, "for sarcastic I usually use a combination of two emoticons to show that I am angry and sarcastic. For example, mad face + crying from laughing". Participants used the same strategy and combined emoticons with acknowledgment and confirmation words in different emotions. For example, they used "I 
know" in combination of sad, happy, or angry face-icon to present their acknowledgment in different emotions.

- Some gesture categories such as "P" were confusing for participants. They suggested that some categories needed to be combined. " $P$ wouldn't come to my mind. But as you explained that, it makes total sense", "I think _ and \ need to be combined. Maybe we could have one category I", " __ and \overlap? After explanation, I know the difference but at the beginning I wouldn't distinguish between them. I would combine both in one category".

- A "being supportive" category was missing. Participants reported they needed a gesture besides heart to specifically empathize and support their friend in different emotions. "Heart is too much if I want just to support someone and give her a hug, I won't draw a heart.", "When I want to do empathy like patting, what gesture should I use? ...maybe heart... but it conveys love...", "What gesture should I use if I just want to support and give her hug?".

- Although participants suggested adding an empathy/support category, they reported that the number of categories was just fine and adding more to the categories would make it complicated and they might not remember. For example, when participants were asked if they liked to have different categories for different intensities emotions (e.g. happy and excited, sad and very sad), their responses were negative. "Having gestures for emotions with different intensity is too much! I use smiley for happy, laughing, or excited", "The number of categories is enough, more than that and III get confused and can't remember them", "If there were too many categories I couldn't remember them", "Maybe we can have 
another category for laughing and lol or those things. But it will get very difficult..."

Interestingly, the main gesture categories that were identified for the sadness and anger responses from co-design sessions were similar with different categories identified for anger and sad responses in brainstorming sessions as they are shown in figure (34).

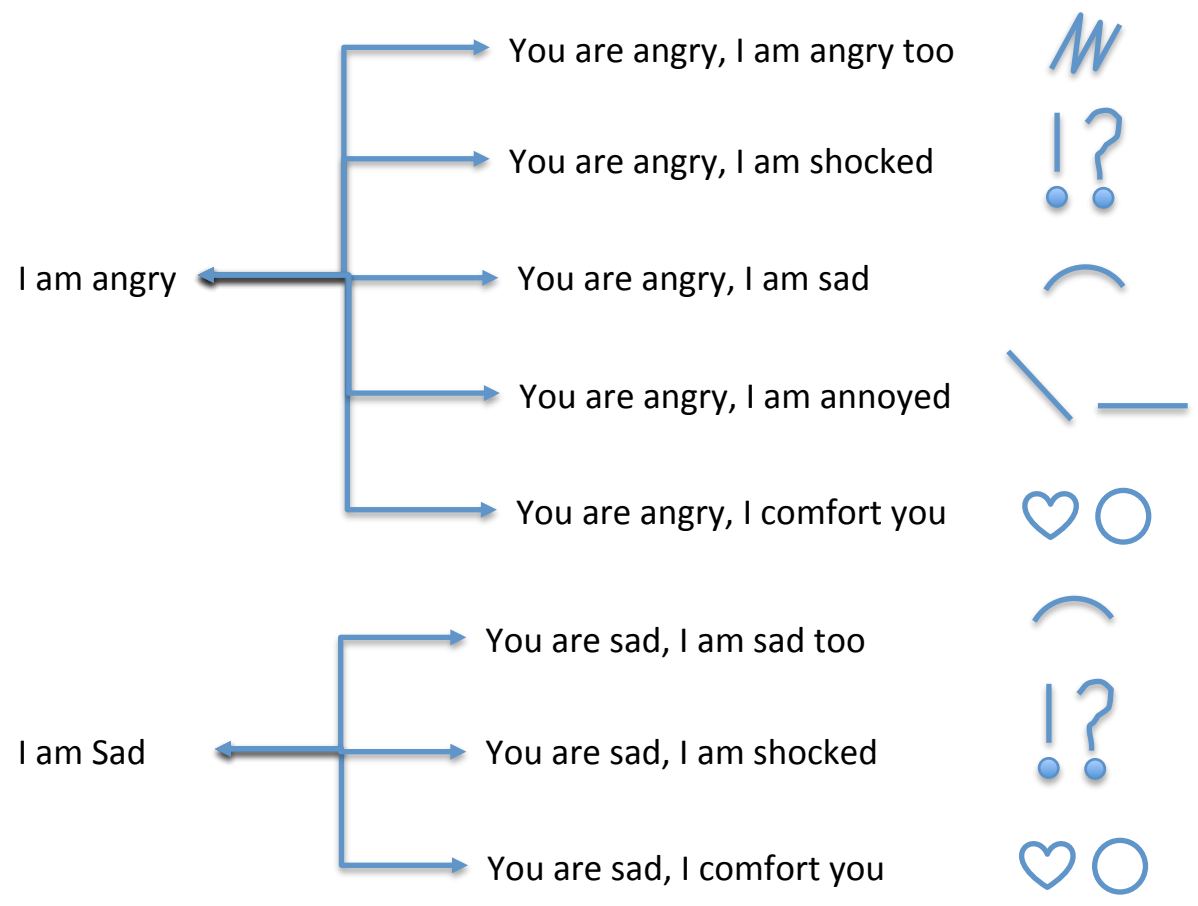

Figure 34. Combining the results of brainstorming sessions and co-design sessions with respect to sad and anger response

\section{Color design solution}

Participants also reported their interest in the color design solution. Especially participants who did not use emoticons, expressed more interest in using this feature, since it was simple and quick to use and presented the emotion clearly. However, participants that used emoticons more often used color as an additional emotional 
representation to emoticons. "I don't use emoticons, the color would work for me. Emoticons are childish and you don't want to use them in serious texting. When its serious, words and color would be the best way to convey my emotion". "all other apps are the same with different looks but this app really helps to show my emotion. I might actually use fewer emoticons if I have colors. But its great to have both color and emoticons", "I like the idea of the color. If you could add color to each gesture category it would be cool! ", "I really like the idea of color. Attaching color to text is cool and can be useful".

Another interesting theme that was identified was the order of changing color and texting. Some participants changed the color first and then text and some texted first and then changed the color of the text background. "I text first and then change the color. I prefer to text and get it off my chest first and then set the color", "I would text first and then pick the red and select some related emoticons", "I prefer to pick the color first. It's good to show on the keyboard somehow that the color has been changed".

Besides the positive feedback that the color solution received, there was also some confusion with this design solution. Agreement on some emotion colors such as red for angry, or yellow for happy was reached quickly. However, participants didn't agree on colors for some emotions like sadness or being sarcastic. Another limitation of this feature was the confusion of this feature overlapping with the gesture design solutions. Since participants could reach related emoticons of the three main emotions of happy, sad, and angry from both the color button and gesture board, they sometimes were confused which one they need to use. Note that, there was no right or wrong way and participants could do both ways.

Recommendation for future application

Participants also suggested a few features for future improvement of the design that could be added to the application: 
- Voice: participants reported voice as a feature that they use in current text messaging applications and could be added to this application too.

- Smart application: developing a smart application that recognizes emotion based on the words was another suggestion reported by participants. "It would be great if the application was smart and could tell me based on what I text which emoticons I need to use" "It would be cool if the app could tell me what emotion I'm having and show me the related the emoticons."

- Colorful stickers and 3D emoticons: some participants, especially those who already used emoticons often suggested to have a variety of colorful and interactive emoticons and body icons.

- Voice intensity: adding a feature to convey the Intensity of the voice was also reported as a suggestion. "Maybe you could have something that if I press the button and hold it longer it shows I am very angry or if I just hit it shows that I'm a little bit angry", "have a color adjustment to say how mad you are with a scroll bar."

- Font change: changing the font in addition to the color was also suggested by participants for conveying different emotions. "There needs to be a sarcastic font. Sarcastic is really hard to get through with words", "Sarcastic should be italic."

- Received text: How participants prefer to receive the text was also explored. Some participants reported they liked to send and receive emoticons bigger than their normal size. For example, "I like the emoticons big so that she understands my emotion better", "I like when the emoticon is bigger because I really feel like the other person has 
some emotion". Some participants, however, mentioned the alignment of text and the emoticon on the screen is important for them and they would prefer to keep the size of emoticons the way there are. For example "Stick to the format we have. I don't like to see the message and emoticon get the whole screen."

\subsection{Design Solutions and prototype refinement}

The design was finalized based on the results of the co-design sessions and input from two visual design students. Figure 35 presents the final list of screens.

\subsection{Discussion}

Although the medium studied in previous studies was desktop instant messaging and the focus of the design process in this study was mobile instant messaging (text messaging), the results of this study confirmed and in some areas, extended the results of upfront studies.

Similar to the results of the previous studies, emoticons and words were identified as two main strategies users used to communicate their emotion in mobile instant messaging. However, the results of this study identified some of the possible explanations for the results of previous studies, and expanded their results by presenting when and why participants prefer words, emoticons, or a combination of both in text messaging among the different emotions of sad, happy, and angry. 


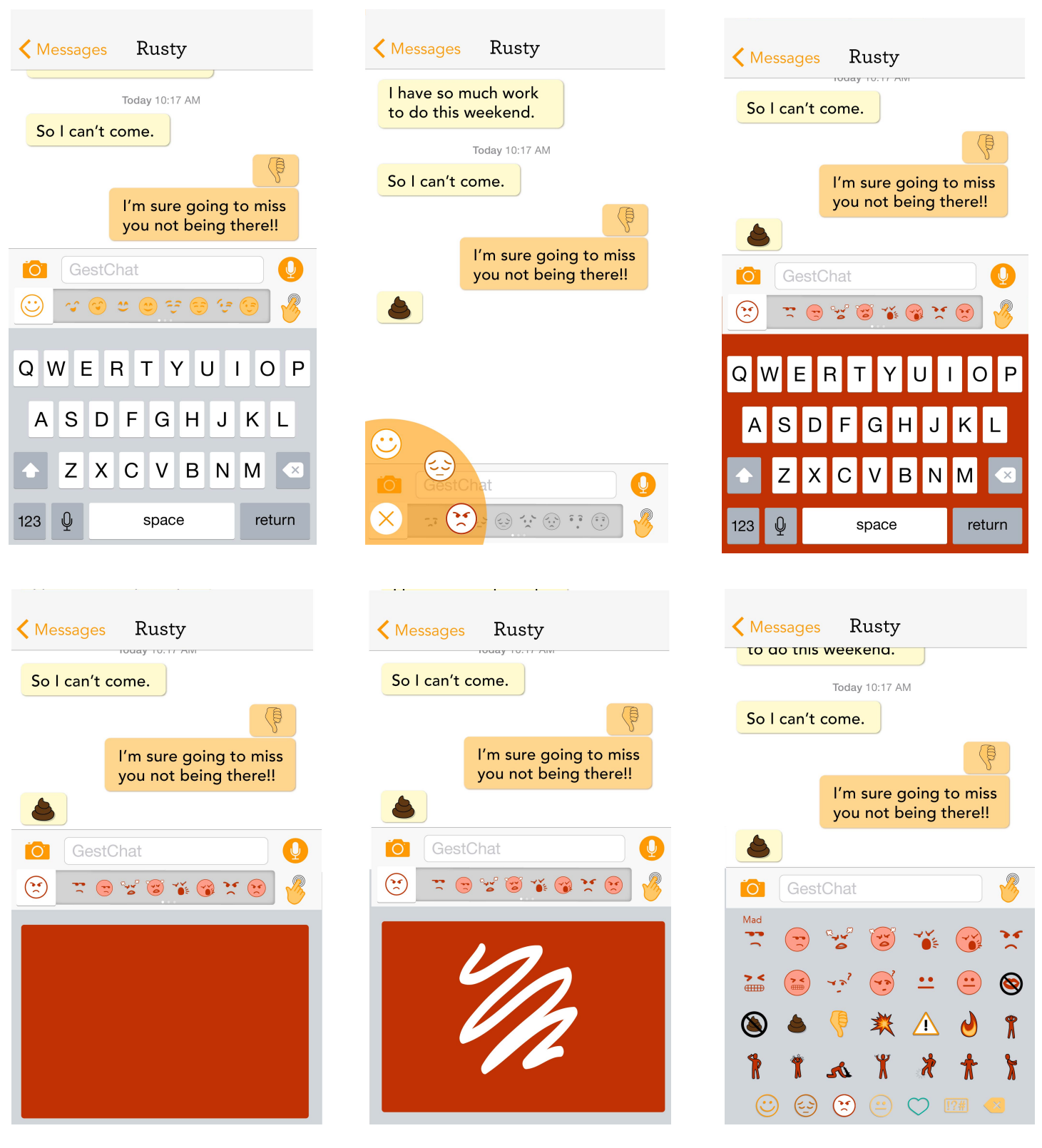

Figure 35. Final design of the color and gesture features

Overall more usage of emoticons was identified for emotional expression and response in mobile instant messaging through the design process compared to the results of the conversation analysis of study III. One possible explanation could be the change of medium from instant messaging to mobile instant messaging. Since participants use mobile instant messaging in everyday life, they might have been more 
used to using emoticons in text messaging. The second possible explanation could be the focus of the design process. Since participants were asked to evaluate emoticons and words, they were more exposed to emoticons and used them more.

Regarding emotional expression, the results of the conversation analysis showed more usage of emoticons in happy expression compared to sad and angry expression. This study could explain those findings in two ways 1) Some participants prefer not to express their negative emotion especially anger, in general, regardless of the medium, because of their culture, background, or personality traits. 2) Current emoticons are cartoony and funny and degrade their emotions, specifically their negative emotions. 3) Emoticons have been overused for positive emotional expression via text messaging, and the lack of emoticons and using only words per se could represent the negative emotion. Therefore, some participants do not see any reason to use emoticons for negative expression.

Regarding the response to emotional expression, the results of study III conversation analysis showed that participants used mostly lexical surrogates of "haha" and "Iol" to respond to happy expression and confirmation cues (e.g. "I know", "right") to respond to sadness and anger. This study identified an explanation for that finding. Participants reported that they preferred words or a combination of words and emoticons to acknowledge sad and anger expression because emoticons or lexical surrogates are not enough to empathize with the negative emotions of their friends. They reported when they used only emoticons it might be a representation of them not caring or empathizing enough. Another theme identified related to the lack of interest in using emoticons in response to negative emotions was the visualization of current emoticons. Similar to emotional expression, some participants reported that emoticons are cartoony/funny and might degrade the importance of their emotion in empathizing with their friend especially in sad and anger. Supporting the results of study III's conversation analysis, participants 
used capital letters, vocal spelling, and punctuation, to mimic the prosody of face-to-face communication in mobile instant messaging.

Two main groups of users were identified in this study. First, participants who were not willing to express their emotion, regardless of the medium and second, participants who were willing to express their emotions. The second group was also divided into two subcategories of participants who were satisfied with current design solutions that support them to communicate their emotion and who were not content with their emotional communication via current text messaging application. This confirmed the results of study III and the importance of personality traits and culture on emotional communication in text messaging.

Text messaging was identified as a fast and convenient medium of communication that should not need too much effort to interact with. Changing the design direction from developing equivalent multi-touch gestures for different words to using gestures as a quick shortcut to a specific group of emoticons was another proof that text messaging is used as a fast medium of communication. This result supported the results of study II in which one of the main features of instant messaging was identified as fast communication with short messages, mainly with those who users already know and have relationships. The results also confirmed other studies in which introducing text messaging as a fast, easy and convenient mode of communication (Grinter and Eldrige, 2001).

Developing user-defined gestures and assigning colors to emotions were two main design solutions that were developed in this study. Although three main emotions of happy, sad, and angry were the main focus of the design process, some additional emotions including surprised/shocked, annoyed, and being supportive/empathy were identified as critical emotions in response to those three basic emotions. 
Since the main goal of the design process was developing and evaluating the design solutions in a low fidelity prototype and the application was not fully implemented, it was not feasible to conduct a formal evaluation to compare the design ideas with the current applications in the market. However, some evaluation criteria were identified from data analysis of the co-design sessions to evaluate the application comprehensively as it is implemented:

- Learnability: How easy is it for users to learn the main features of the application (gestures and color) and reach needed emoticons and words to add to the text for the first time they encounter the design, compared to the current applications?

- Efficiency: Once users have learned the main features of the application and got familiar with the design, how quickly they reach different words and emoticons they need.

- Memorability: When users return to the design after a period of not using it, how easily can they reestablish proficiency?

- Control: how much users feel in control using the main features of the application, compared to the current application.

- Errors: How many errors do users make, how severe are these errors, and how easily can they recover from the errors?

- Satisfaction: How pleasant is it to use the design? And specifically how pleasant are the new emoticons.

- Utility: whether the application provides the feature they need?

- Emotional expressivity: whether they use more emoticons? And whether they become more expressive drawing the symbol to get the emoticons? 


\subsection{Implication for technology and design}

This study has implications for both multi-touch technology and user interface design. Multi-touch gestures that were identified in this study have implications for surface recognition technology, especially mobile technology. Since users use their cellphones on the go, moving forward with multi-touch technologies, special attention should be paid in recognizing gestures while users are moving and interacting with their phone considering the small size of cellphone screens.

Identifying different groups of users with respect to emotional communication in text messaging will also provide helpful insights for designers to understand mental models of each group of users and customize their design based on their target users.

Insights gained in this study on when, how, and why users use words and emoticons in text messaging could also have significant implications for designers to understand users better and design applications that satisfy users need. Considering text messaging as a fast communication revealed efficiency and utility as the main criteria for designers to evaluate their design ideas related to text-messaging applications.

\subsection{Conclusion and future work}

This study presented the design process that was conducted including an active process of ideating, iterating, and critiquing potential multi-touch gesture based design solutions to support emotional communication in mobile instant messaging. The design process was grounded in real knowledge produced by upfront research (study I, II, and III) on how people actually communicate their emotions in IM.

The usage of emoticons and words in emotional communication in text messaging was investigated in this study. Extending and confirming the results of studies I, II, and III, this study explored more in depth why and when users apply words 
and/or emoticons to express and respond emotion in different emotional states of happy, sad, anger.

A set of seven user-defined multi-touch gestures were also developed as shortcuts to different categories of emoticons and words related to sad, happy, angry, annoyed, surprised/shocked, empathy, and love. The user-defined set of gestures has properties that make it a good candidate for deployment in instant messaging mobile applications.

In the design process of developing the gestures, insights into the mental models of mobile instant messaging users were gained and translated into implications for design and technology However, there were several limitations that need to be addressed in future studies.

The medium that was studied in this study was mobile instant messaging. Future studies need to explore other text-based communication media such as instant messaging and email and also other platforms such as laptop or tablets.

The design process explored design solutions that were mostly focused on facial expression and tone of voice in text messaging using emoticons and lexical surrogates. Future studies need to explore design solutions to support emphasizing certain words in a statement or expressing the intensity of the emotion. Participants use capital letters, vocal spelling, and punctuation to convey intensity and emphasis on specific words. Future studies also need to expand the range of emotions and explore emotional expression and response in other emotional states such as fear, or boredom.

Participants participated in this study were students who were technologically savvy and used text messaging in every day communication. Future studies should be replicated by more diverse participants in different age groups and with varying familiarity with technology and text messaging. 
Finally, since the application was developed in the low fidelity prototype level to mainly communicate, evaluate, and refine the design ideas with participants, the final evaluation and comparison of the application with the current applications in the market were not possible. Future studies need to implement the application to evaluate the design ideas and compare them with the current design solutions. 
Chapter 7. Conclusion and Contributions

Emotional communication is fundamental to everyday interaction. How well emotions are communicated is crucial to interpersonal relationships and individual wellbeing (Fussell, 2002). In spite of the rapid growth of text-based instant messaging (IM) in diverse settings, emotional communication in IM has received limited empirical scrutiny, especially inside casual settings.

Connecting bodies of literature in text-based computer-mediated communication and design, through a design research approach, this dissertation critically examined how IM users communicate emotions and established multi-touch gesture-based design solutions to support emotional communication via this medium. The design research included three upfront studies (I, II, and III) on how people actually communicate their emotion in instant messaging and a design process that was conducted to explore gesture-based design solutions to support emotional communication in mobile text messaging.

Study I extended previous studies on emotional communication in CMC (e.g. Boonthanom, 2004; Hancock et al., 2007; Hancock et al., 2008) by exploring the effect of two psychological states (mood and stress) on the type and quantity of emotional cues users apply in task-focused IM communication. The results of this study showed that participants under stress produced significantly more self-references, negative emotions, and cognitive words, but fewer vocal spellings than non-stressed participants. There was also a significant correlation between extraversion and several of the chat cue categories (e.g. vocal spelling, lexical surrogates, minus features, social words, positive emotion words, and articles). This study provided empirical support for Brunswik's lens model (1956) in synchronous text-based CMC, demonstrating that situational context and personal traits of the encoder can affect emotional communication. 
Although study I presented an initial effort to understand emotion expression in text-based CMC, several insights were gained to design study III. Study III needed to cultivate controlled emotionally-laden situations in which participants are more likely to apply text-based emotional message cues to express their emotions, rather than opportunistically mine data sets such as these, which are more task-focused than expressly intended to elicit emotional exchanges between partners. Study III also needed to operationalize emoticons in addition to strictly text-based emotional message cues. Study I provided empirical support for Brunswik's lens model (Brunswik, 1956) in text-based CMC, study III also needed to apply the modified version of the lens model to characterize the influences of other emotional states (relaxed, angry, happy, sad), on the proportions of different emotion-related cues used during informal IM conversations in controlled emotionally-laden situations.

While Study I mined communication data to better understand how people express emotions using existing text-based emotional cues and provided several insights for Study III, Study II as an exploratory design research was conducted to investigate the potential of multi-touch gestures to support emotional communication in this medium. The results of study II showed how participants perceived and reacted to multi-touch gestures for emotional expression in IM and revealed some positive feedback from participants in interacting with gestures. This study provided evidence that multi-touch gestures have the potential to support emotional communication in IM.

Study III was conducted to address some limitations of study I and other previous studies (e.g. Boonthanom, 2004; Hancock et al., 2007; Hancock et al., 2008) in the area of emotional communication in instant messaging and extend their findings. Similar to study I, study III also applied the modified version of the Brunswik's lens model. This study investigated how people actually communicate their emotions during informal IM conversations in controlled emotionally-laden situations through a mixed-methodology 
approach. The quantitative analysis investigated how different emotional states influence cues individuals apply in instant messaging. While qualitative analysis investigated emotion in interaction and identified emotional cues IM users used in emotional communication including emotional expression and response via this medium.

Similar to study I, the results of study III (quantitative and qualitative) provided empirical support for Brunswik's lens model (1956) in synchronous text-based CMC, demonstrating that the emotional state of the encoder can affect emotional communication and the usage of text-based cues in IM. The results of study III's, quantitative analysis, revealed that the happy condition led to more use of nonverbal cues than the other three conditions, including more punctuation, vocal spellings, lexical surrogates, and minus features. This increase in nonverbal emotional expressivity led to three potential explanations. The first is that $I M$, as it is currently implemented in common desktop and web-based applications such as Google Chat, may not support negative expressivity sufficiently. This explanation was in line with the survey results, in which some of the participants reported that sadness and anger were difficult to express in IM because of the lack of cues to communicate facial expression in this medium. This explanation, inline with social presence theory, put the social information processing (SIP) theory in a new perspective with respect to negative emotions, since the results showed that users were not able to find any strategies to convey nonverbal behaviors to express their negative emotions. The second explanation is that participants expressed negative emotions using cues, not among those captured in this analysis. The results of qualitative analysis provided some additional evidence for this explanation. Study III's qualitative analysis showed that the main strategy receivers used to acknowledge emotional expression and empathize with senders in sad and angry conditions were agreement cues (specifically "yeah" and "I know"), which were not counted in the quantitative analysis. Lastly, the third explanation is that perhaps sad and angry partners 
chatting informally turn their focus inward and become less expressive overall. This explanation was in line with the survey results, in which some participants reported they wanted to focus more on the reason that made them angry before expressing their anger.

Finally, the design process was conducted based on the results of upfront research (study I, II, and III). The design process involved an active process of ideating, iterating, and critiquing potential multi-touch gesture based design solutions to support emotional expression and response in instant messaging. The results of this study provided some evidence to support the results of study III.

The results of the design process revealed that users in sad and angry emotional states sometimes turn their focus inward and become less expressive overall. Participants also reported that they prefer words or combinations of words and emoticons to acknowledge sad and anger expressions because emoticons or lexical surrogates are not enough to empathize with the negative emotions of their friends. Another theme identified related to the lack of interest in using emoticons in response to negative emotions was the visualization of the current emoticons. Similar to emotional expression, some participants reported that emoticons are cartoony/funny and might degrade the importance of their emotion in empathizing with their friend especially with sad and anger. These results provided more evidence for the argument on why users used less nonverbal cues in sad and angry conditions compared to happy condition.

Armed with the results of studies I, II, III, and IV, there were also several limitations throughout these studies that will need to be addressed in future studies. Study III explored the topic by cultivating emotionally-laden situations by using video clips and memory elicitation techniques. Future studies need to apply other creative techniques to increase the emotion expression in natural conversation among friends.

Based on the results of study I, extraversion (Scherer, 1978) and emotional intelligence (Petrides and Kokkinaki, 2007) could be the most likely factors to explain 
some of the variation between individuals in their use of verbal and non-verbal cues to express and respond to emotion in IM, and should be considered in future research in this area.

Future studies also need to study text-based CMC beyond emotional expression to explore different categories of cues and activities that individuals use to connect and develop social bonds such as affinity, commitment, and attention.

Participants who participated in study III and IV were students who were technologically savvy and used text messaging in every day communication. Future studies should be replicated by more diverse participants in different age groups and with varying familiarity with technology and text messaging. Future studies also should reexamine the results of this dissertation in other languages and cultures.

The medium that was studied in study IV was mobile instant messaging. Future studies need to explore other text-based communication media such email and also other platforms such as laptop or tablets.

The design process explored design solutions that were mostly focused on facial expression and tone of the voice in text messaging using emoticons and lexical surrogates. Future studies need to explore design solutions to support emphasizing certain words in a statement or expressing the intensity of the emotion. Future studies also need to expand the range of emotions and explore emotional expression and response in other emotional states such as fear, or boredom.

The design research that was conducted through this dissertation has several contributions. The first contribution is identifying gaps in theories and models of emotional communication in $\mathrm{IM}$ and bridging the general aspects of emotional communication to a specific context of informal IM conversations.

The results of studies in this dissertation provided empirical support for Brunswik's lens model (Brunswik, 1956) in text-based CMC, which argues that 
situational context and personal traits of the encoder can affect emotional communication. The results also support SIP theory with respect to positive emotion, in which participants discover different strategies to adjust positive emotional communication to IM. However, regarding sadness and anger communication, the results put SIP theory in a new perspective and support social presence theory, in which despite two different strategies users identified to communicate negative emotions, they still encounter challenges to communicate negative emotions via this medium.

To summarize the first contribution, the results of this dissertation revealed that the medium of instant messaging alone does not determine whether there are limitations with users' emotional communication. Difficulty in emotional communication via instant messaging varies among different users, emotional states they are communicating in, and supporting design solutions available for them. Some users are satisfied with emotional communication via current applications (with/without using emoticons) and some still experience difficulties. Emotional communication also varies among different emotional states.

The second contribution of this dissertation is presenting a holistic research contribution that reveals the balance researchers have between conflicting perspectives of communication, psychology, design, and $\mathrm{HCl}$. Upfront, studies I, II and III and the design process reflected how knowledge was created throughout the dissertation with the design research approach. This dissertation also showed how different studies, supported and complemented each other's findings.

The third contribution of this dissertation is providing inspiration and motivation for designers to design and engineers to build new technologies for IM applications to support emotional communication (See appendix $G$ for more details on the patent which was filed on emotional communication in text messaging using multi-touch gestures). This dissertation specifically focused on gesture-based design solutions to support 
emotional communication in text messaging. A set of ten multi-touch gestures was developed as a shortcut to different categories of emoticons and words. Based on the results of this project, a new set of emoticons was also designed. Nowadays, more and more companies (e.g. Facebook, Google, and Yahoo) realize the importance of emotional communication in IM and other social network applications. The results of this dissertation could provide inspiration for the new direction different companies take on supporting emotional communication.

Finally, the fourth contribution is that the artifacts provided by this design research reflects on theories and technical opportunities and could be used in $\mathrm{HCl}$ or design practice communities. The output of this study could transfer knowledge to different communities of research, practice, and education. 
Appendices

Appendix A: Demographic questionaire

Demographic Information

Gender: __ Male _ _ Female

Age:

Educational Level

Bachelor Student

Master Student

PhD student

other

Race

_. American Indian or Alaska Native

Asian

Black or African American

- Hispanic or Latino

Native Hawaiian or Other Pacific Islander

White

Other

Are you a native English speaker?

Yes

No

For how long you now your partner in this experiment.

_ Less than 6months _ _ 6 months to one year _ _ one year to three years _ _ More than three years

On average, how often do you chat with following devices?

\begin{tabular}{|c|c|c|c|}
\hline $\begin{array}{l}\text { Almost } \\
\text { Every day }\end{array}$ & $\begin{array}{l}\text { few times } \\
\text { each week }\end{array}$ & $\begin{array}{l}\text { between once a week } \\
\text { and once in a month }\end{array}$ & $\begin{array}{l}\text { Less than once } \\
\text { in a month }\end{array}$ \\
\hline
\end{tabular}

Computer/Tablet

Cellphone/Smart phone

If you chat via computer/tablet how often you use the following software to chat

$\begin{array}{llll}\text { Almost A } & \text { few times } & \text { between once a week } \\ \text { Every day } & \text { each week } & \begin{array}{l}\text { Less than once } \\ \text { and once in a month }\end{array} & \text { in a month }\end{array}$

Google Chat

Yahoo Messenger

AOL Instant Messenger

Other 


\section{Appendix B: Emotion manipulation check survey}

\section{Dear Participant:}

This scale consists of a number of words that describe different feelings and emotions. Read each item and then mark the appropriate answer in the space next to that word. Indicate to what extent you felt this while watching the film clip. Use the following scale to record your answers.

\begin{tabular}{|c|c|c|c|c|}
\hline 1 & 2 & 3 & 4 & 5 \\
\hline $\begin{array}{c}\text { Very slightly or } \\
\text { not at all }\end{array}$ & A little & Moderately & Quite a bit & Extremely \\
\hline
\end{tabular}

Happy

Annoyed

Afraid

Tense

Satisfied

Bored

Calm

Droopy

Astonished

Aroused

Gloomy

Relaxed

Alarmed

Excited
Angry

$\mathrm{Sad}$

Glad

Pleased

Tired

Sleepy

At ease

Serene

Frustrated

Content

Delighted

Depressed

Distressed

Miserable

Did you feel any other emotion during the film clip?

-------Yes --------No

If so what was the emotion?

How much of this feeling did you feel? 


\section{Appendix C: Sample questions}

\section{First video clip:}

- Her/his feeling about the video?

- Things she/he usually does to get relaxed?

- Relaxing time she/he has had recently? What did she/he do?

- Does she/he practice yoga or any type of medication techniques? If so what are those?

- What are the differences between relaxing in nature and walking on the beach? Which one does she/he prefer?

- An exercise she/he does to relax her/his body

- Why does she/he need to relax sometime?

\section{Second video clip:}

- Her/his feeling about the video?

- Part of the video made her/him angry the most? Why?

- Any experience that she/he, any friend or family members have been bullied? How?

- Any similar bully scene from other movies?

- If she/he were the movie character who was bullied what would she/he do?

- The recent thing that made her/him angry?

- In general what makes her/him angry the most? Why?

- Type of people that makes her/him angry?

\section{Third video clip:}

- Her/his feeling about the video

- Part of the video made her/his laugh the most

- A funny story from her/his childhood

- The funniest thing that has happened to her/his recently

- The funniest joke she/he has heard

- Things make her/his happy the most. Why?

- The funniest scene of a movie she/he has ever seen

\section{Forth video clip:}

- Her/his feeling about the video?

- A sad memory from losing someone?

- A sad memory from childhood?

- The saddest moment in her/his life?

- The saddest thing that she/he has heard recently?

- Things make her/him sad in general? Why?

- Things about the world these days that makes her/him sad?

- The saddest movie scene she/he has ever seen?

- Things she/he does when she/he gets sad? 


\section{Appendix D: Open ended survey}

Thanks for participating in our experiment. We would be thankful if you could also answer some final questions. You may continue on the back of the page if you need more room.

1. How satisfied were you in your ability to express emotions in text-based chat during the experiment? (Circle one)

\begin{tabular}{|c|c|c|c|c|c|c|}
\hline $\mathbf{1}$ & $\mathbf{2}$ & $\mathbf{3}$ & $\mathbf{4}$ & $\mathbf{6}$ & $\mathbf{7}$ \\
\hline $\begin{array}{c}\text { Totally } \\
\text { Satisfied }\end{array}$ & $\begin{array}{c}\text { Very } \\
\text { Satisfied }\end{array}$ & $\begin{array}{c}\text { Somewhat } \\
\text { Satisfied }\end{array}$ & $\begin{array}{c}\text { Neither } \\
\text { satisfied nor } \\
\text { dissatisfied }\end{array}$ & $\begin{array}{c}\text { Somewhat } \\
\text { Dissatisfied }\end{array}$ & $\begin{array}{c}\text { Very } \\
\text { Dissatisfied }\end{array}$ & $\begin{array}{c}\text { Totally } \\
\text { Dissatisfied }\end{array}$ \\
\hline
\end{tabular}

Explain why:

2. What differences do you see between expressing your emotions in text-based chat compared to expressing them face-to-face?

3. What would be your ideal way to express emotions in text-based chat? 


\section{Appendix E: Brainstorming session plan}

\section{Warm-up}

The main topic of this brainstorming session is emotion and how we communicate our emotion in everyday life. As a warm up activity I want you to close your eyes and think about any memory you remember that made you so happy and gave you joy. Later in this session I will need you remember this memory.... so grab a yellow post-it and write down a few words of the memory, the approximate date or time or anything that would help you remember the memory later through this session.

Now close your eyes again and try to remember a sad memory in your life. Grab a blue post-it and write down something that help you remember this memory later in the session.

We are going to repeat this memory elicitation for anger emotion. Close your eyes and think about an experience in your life that made you very angry. Write it down on a red post-it this time.

\section{Introduction}

So why did we do this memory elicitation? I'm working on a project on emotional communication in instant messaging. I did an experiment with 20 students in 10 pairs in informatics school. We put them in different rooms and showed them 4 videos to elicit four emotions of relaxed, happy, sad, and angry. Then we asked them to chat with their friends via instant messaging. We did a quantitative analysis and found that people use more emotional cues including emoticons, vocal spelling (e.g. sooo, weeell), lexical surrogates (e.g. uh huh, haha), and abbreviations (e.g. Idk) in happy condition than in sadness and anger communication. From the survey questions participants explained that it's hard for them to communicate their negative emotions since there isn't sufficient support of negative emotional communication in IM applications. However, Some participants reported that they prefer to turn their focus inward when they feel negative and become less expressive overall.

The focus of my study is to support IM users who want to communicate their negative emotions and figure out how we can support them. We are using happiness communication as a point of reference since users reported they are satisfied in communicating their happiness.

We also did a qualitative research and found that not only emotion expression is important, but also emotion acknowledgement is critical in emotional communication in IM. People use different strategies to empathize and sympathize with their friends in IM.

So for this session, we are specifically interested to see how you communicate (expression and acknowledgement) sadness, anger, and happiness in face-to-face and how you transfer that to the paper, which represent instant messaging medium.

\section{Activities}

You are going to work in groups of two. We have four cameras around the room that record the session. Find a camera for your team since we want to record whatever you do in this session in case we need them later.

We will start with sadness. Imagine yourself in the past when that memory (on the blue post-it) happened to you and act like you are explaining that incident to your friend. While one person expresses his/her emotion the other partner tries to empathize with her/him.

After that you have 3 minutes to transfer your emotion expression to your paper forms. Imagine now your friend is far away from you and you have to communicate your emotion through instant messaging. Your partner will also transfer his/her empathy to the paper. 
The only thing is that we don't want you to use words. We are specifically interested in nonverbal channels. We have different channels on your form that you need to specifically transfer the emotion through those channels separately.

We need you to come up with at least two ideas for each expression and acknowledgment. We also have intensity factor. So we need you to think about how would you communicate your emotion when it has high intensity? For example when you are very sad or angry.

In each box there is a small box that you need to give us a number from 1 to 5 on how confident you think you communicated that emotion in the picture you draw. 1 the least confidence and 5 the highest confidence.

After that you switch your role sand the expresser plays the role of empathizer. 
Appendix F: Brainstorming session forms

\section{Sad-Emotional expression}

\section{Touch-based gesture}

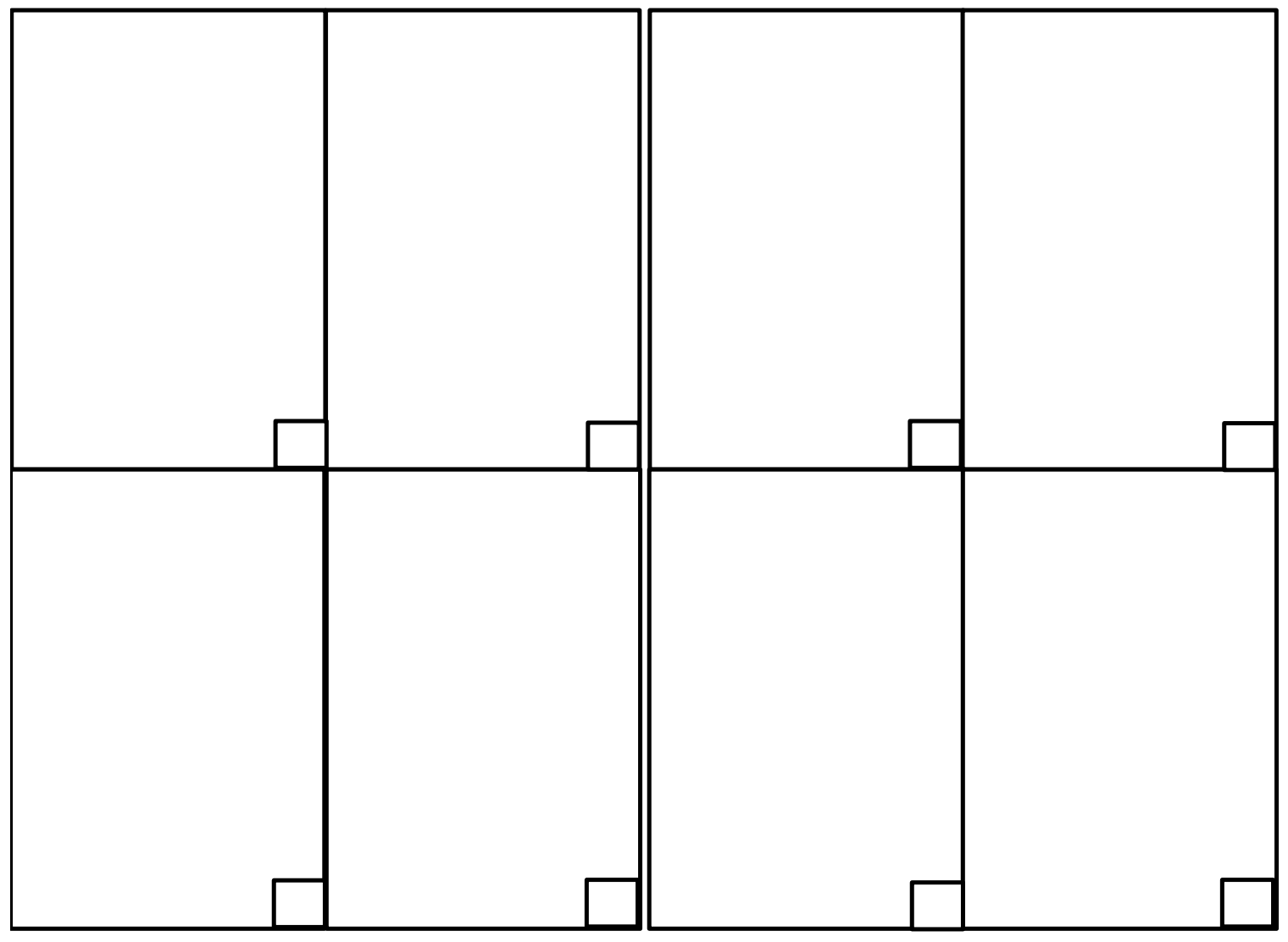


Sad- Emotional expression

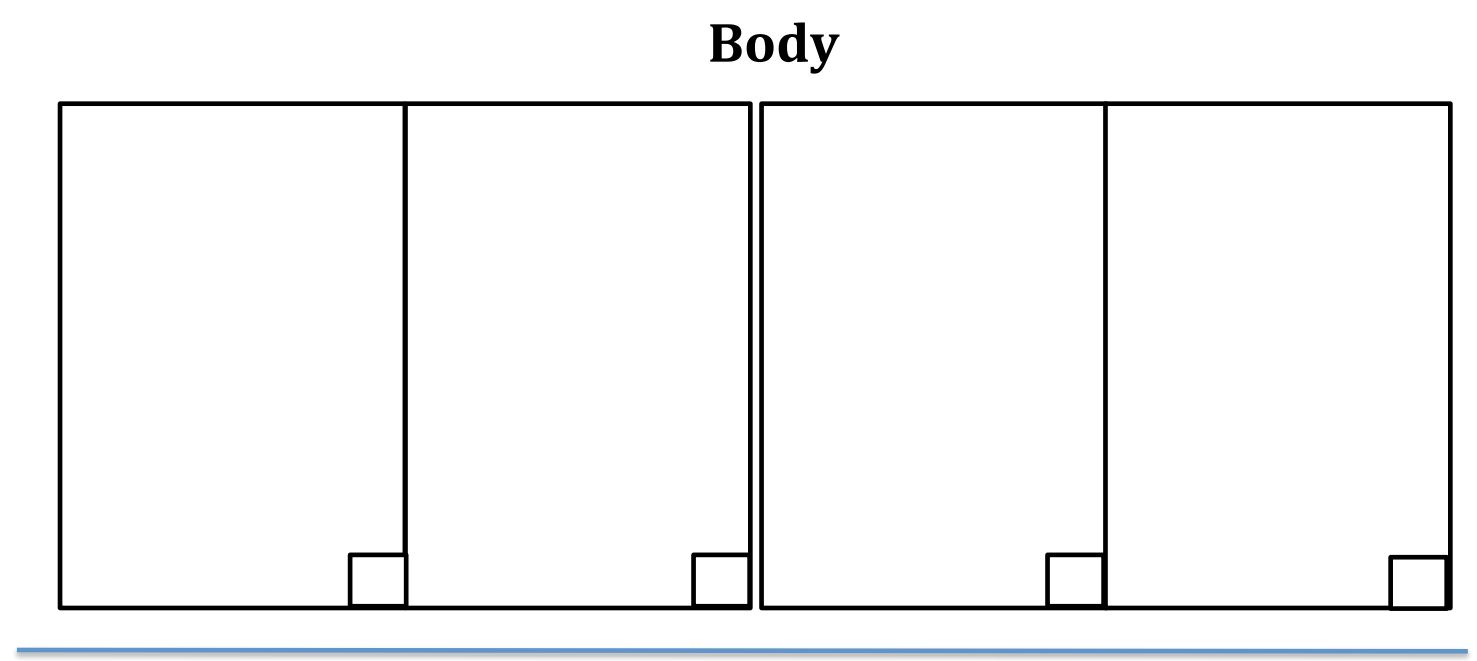

Sign/Symbol

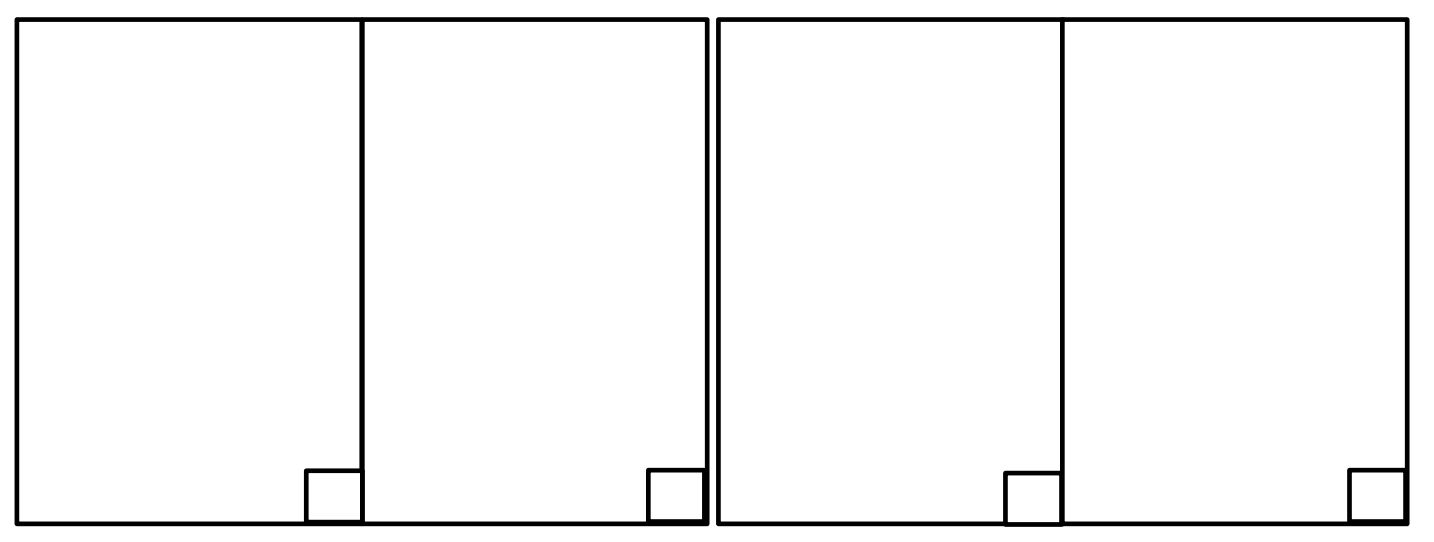

\section{Lexical Surrogates}

(textual representations of vocal sounds that are not words, e.g. uh huh, haha)
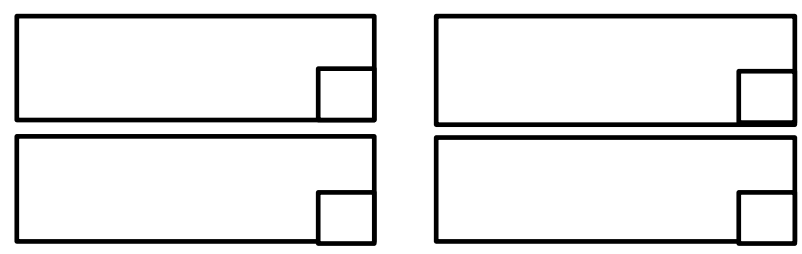
Sad- Emotional response

Body

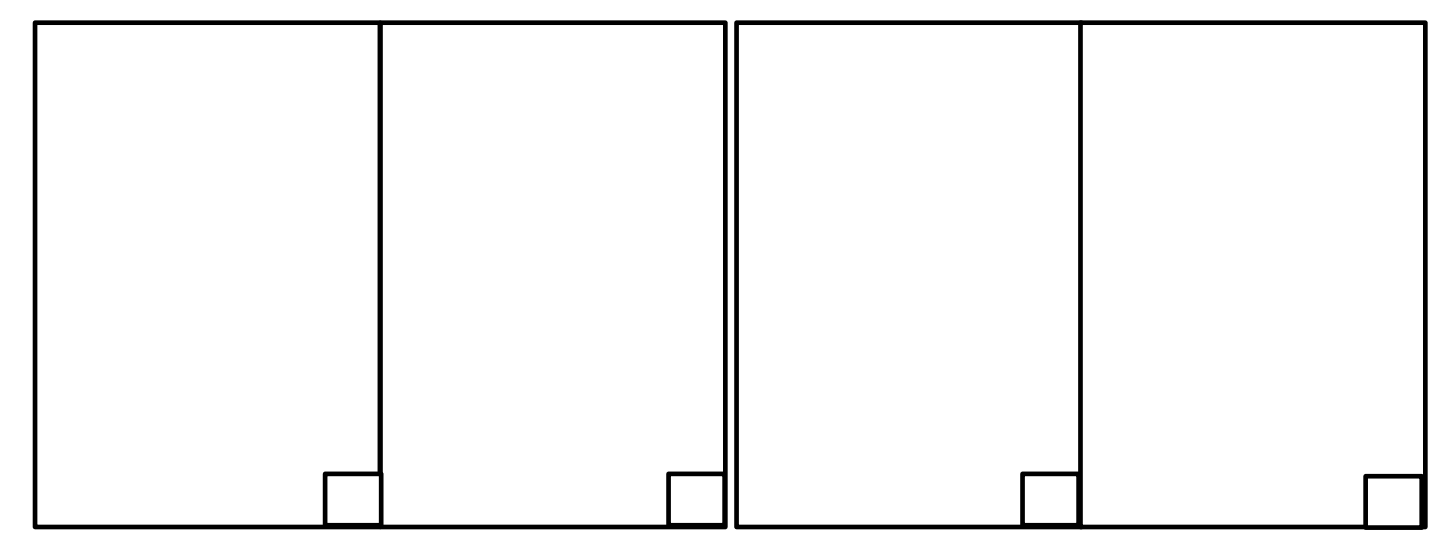

Sign/Symbol

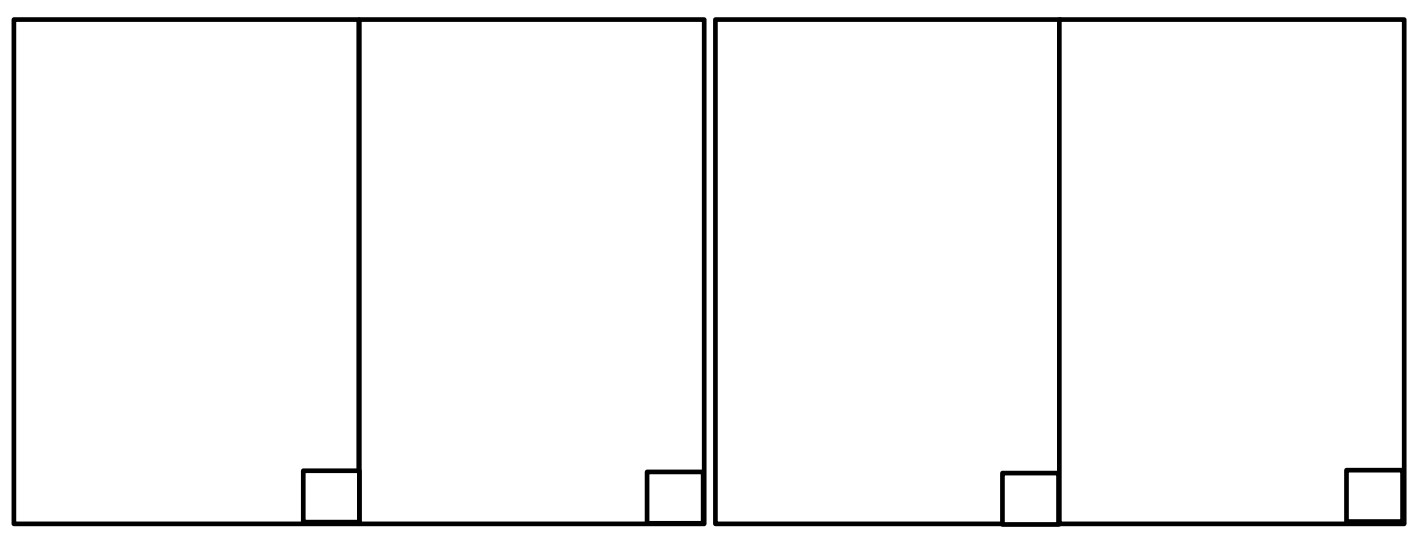

\section{Lexical Surrogates}

(textual representations of vocal sounds that are not words, e.g. uh huh, haha)
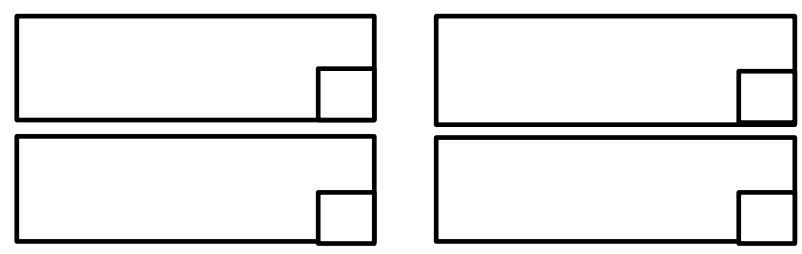


$$
\overline{\bar{I}}
$$


Appendix G: Emotonal gesture-based communication method patent

PROVISIONAL PATENT APPLICATION

of

AFARIN PIRZADEH

for

EMOTIONAL-GESTURE BASED COMMUNICATION METHOD

Attorney Docket No.: IURTC-2015-124-01-US-E

IURTC Reference No.: 2015-124

US.56112227.01 
ATTORNEY DOCKET: IURTC-2015-124-01-US-E

\section{EMOTIONAL-GESTURE BASED COMMUNICATION METHOD}

\section{FIELD OF THE DISCLOSURE}

[0001] This disclosure relates to a method for communicating emotions via text messaging, more particularly, this disclosure is related to a method of instant messaging to input emotional cues through touch and motion gestures on a first side and present them through visualization of the gestures, face icons, body icons, sign/symbols or vibration on a second side.

\section{BACKGROUND OF THE DISCLOSURE}

[0002] Technology has revolutionized the way people communicate. People use a variety of media to enhance and extend interpersonal communication depending on social, security, or efficiency factors. Communications media, however, affects the quantity and quality of the messages and can change senders' and receivers' behavior and attitudes. Instant messaging ("IM"), as one type of synchronous text-based computer-mediated communication ("CMC"), is not an exception. Despite the advantages of IM communication (e.g. convenience, mobility, and control) over face-to-face communication, the absence of visual and aural nonverbal cues in IM causes some limitations on users' emotional communication via this medium. IM users sometimes have difficulties expressing their emotions accurately and fail to accurately understand the actual emotion coming from their partners due to the absence of visual and aural nonverbal behaviors. For example, in everyday communications only $7 \%$ of peoples' emotional communication stemmed from the words spoken, whereas $38 \%$ was attributed to verbal tone and $55 \%$ was related to facial expression.

[0003] Prior art instant messaging applications do not include adequate methods for communicating visual and aural nonverbal cues. The absence of visual and aural nonverbal cues in IM and its limits on emotional communication presents a variety of new challenges and opportunities for designers and researchers in the area of human-computer interaction ("HCl") and design. The dramatically increasing use of text-based chat for interpersonal communication in everyday life has led to development of several design solutions to address the lack of visual 
ATTORNEY DOCKET: IURTC-2015-124-01-US-E

and aural nonverbal behaviors. As such a variety of prior art methods have been designed which seek to support users in emotional and social communication in IM, these methods include:

Emoticons: Emoticons include visual cues which are used in the absence of nonverbal cues such as gestures and facial expression. Emoticons are designed to indicate a sender's mood or feeling.

Avatars: Avatars may be integrated with IM applications that use automated facial expression recognition to display emotion to a chat partner by an expressive avatar. In other IM applications, the emotion of the user may be detected by analyzing the emotional content of the text and automatically transferring the emotional content to an avatar.

Haptics: Haptic IM uses waveforms with different frequencies, amplitudes, and durations, to which emotional meaning can be attached and transferred by haptic devices such as joysticks and/or a touchpad.

Dynamic Typography: Kinetic typography includes a real time modification of text, such as font, color, and size, in IM that can be used to communicate a user's emotion.

[0004] The above identified prior art methods include one or more designs and/or cost limitations relied upon to improve the communication of visual and aural nonverbal cues during text-based communication. However, the above prior art methods fail to adequately improve emotional and social communication via the text-based communication medium. Accordingly, a need exists for an instant messaging application incorporating one or more methods which improve emotional communication through instant messaging in an efficient and cost effective manner.

\section{SUMMARY OF THE DISCLOSURE}

[0005] This present disclosure relates to a method for communicating emotions during a text-based communication session between at least a first user and a second user; more particularly, this disclosure is related to a method in an instant messaging text-based 
ATTORNEY DOCKET: IURTC-2015-124-01-US-E

communication session wherein a first user inputs emotional cues through touch and motion gestures on an electronic gesture board and transmits the inputted emotional cues to a second user wherein the second user receives an image providing a visualization comprising gestures, face icons, body icons, sign/symbols or vibration which correspond to the emotional cue inputted by the first user via the electronic gesture board.

\section{BRIEF DESCRIPTION OF THE DRAWINGS}

[0006] The above-mentioned and other features of this disclosure and the manner of obtaining them will become more apparent and the disclosure itself will be better understood by reference to the following description of embodiments of the present disclosure taken in conjunction with the accompanying drawings, wherein:

[0007] FIG. 1 shows a main screen of a text-based communication session in accordance with the present disclosure;

[0008] FIG. 2 shows a main screen of a gesture board of a text-based communication session in accordance with the present disclosure; and

[0009] FIGs. 3 - 21 show exemplary gestures from a gesture dictionary in accordance with the present disclosure.

\section{DETAILED DESCRIPTION OF EMBODIMENTS}

[00010] The embodiments disclosed herein are not intended to be exhaustive or to limit the disclosure to the precise forms disclosed in the following detailed description. Rather, the embodiments were chosen and described so that others skilled in the art may utilize their teachings.

[00011] The method and system of the present disclosure centers around new strategies to support emotional communication via instant messaging. In one exemplary embodiment the present disclosure allows IM users to express different emotional cues through touch and motion gestures and visualizes those gestures for their IM partner in different formats of image

US.56112227.01 
ATTORNEY DOCKET: IURTC-2015-124-01-US-E

(including gesture icon, face icons, body icons, signs/symbols, or vibration). The present disclosure specifically introduces a new mode of gesture to instant messaging keyboard and also a dictionary of touch and motion gestures of emotional cues to support emotional communication in this medium. From the sender's point of view, as soon as an IM user selects the gesture mode (by hitting a key or doing a specific gesture like a vertical swipe on the screen) (Fig. 1) a gesture board is presented on the screen that makes the user able to input a touch or motion gesture (Fig. 2). The user is able to add a gesture on the gesture board or move the phone through a motion gesture, receive feedback on the screen to review the gestures he/she entered, and send the gesture by hitting the send button or specific gesture (tapping the screen or shaking). From the receiver's point of view, as soon as the sender sends the gesture, the receiver can receive the gesture in different formats of the gesture icon, emoticon (face icon, body icon, sign/symbol), customized image or vibration.

[00012] In the foregoing specification, specific embodiments of the present disclosure have been described. However, one of ordinary skill in the art will appreciate that various modifications and changes can be made without departing from the scope of the disclosure as set forth in the claims below. Accordingly, the specification and figures are to be regarded in an illustrative rather than a restrictive sense, and all such modifications are intended to be included within the scope of disclosure. The benefits, advantages, solutions to problems, and any element(s) that may cause any benefit, advantage, or solution to occur or become more pronounced are not to be construed as critical, required, or essential features or elements of any or all the claims. The disclosure is defined solely by the appended claims including any amendments made during the pendency of this application and all equivalents of those claims as issued. Although the invention has been described in detail with reference to certain preferred embodiments, variations and modifications exist within the spirit and scope of the invention as described and defined in the following claims.

US.56112227.01 
ATTORNEY DOCKET: IURTC-2015-124-01-US-E

\section{CLAIMS}

1. A method for communicating emotions during a text-based communication session between at least a first user and a second user, comprising:

inputting, by at least the first user, one or more emotional cues through touch and motion gestures on an electronic gesture board; and

transmitting the inputted emotional cues to the second user wherein the second user receives an image providing a visualization comprising at least one of gestures, face icons, body icons, sign/symbols or vibration which corresponds to the emotional cue inputted by the first user via the electronic gesture board.

US.56112227.01 
ATTORNEY DOCKET: IURTC-2015-124-01-US-E

\begin{abstract}
This present disclosure relates to a method for communicating emotions during a textbased communication session between at least a first user and a second user. More particularly, this disclosure is related to a method in an instant messaging text-based communication session wherein a first user inputs emotional cues through touch and motion gestures on an electronic gesture board and transmits the inputted emotional cues to a second user wherein the second user receives an image providing a visualization comprising gestures, face icons, body icons, sign/symbols or vibration which correspond to the emotional cue inputted by the first user via the electronic gesture board.
\end{abstract}

US.56112227.01 


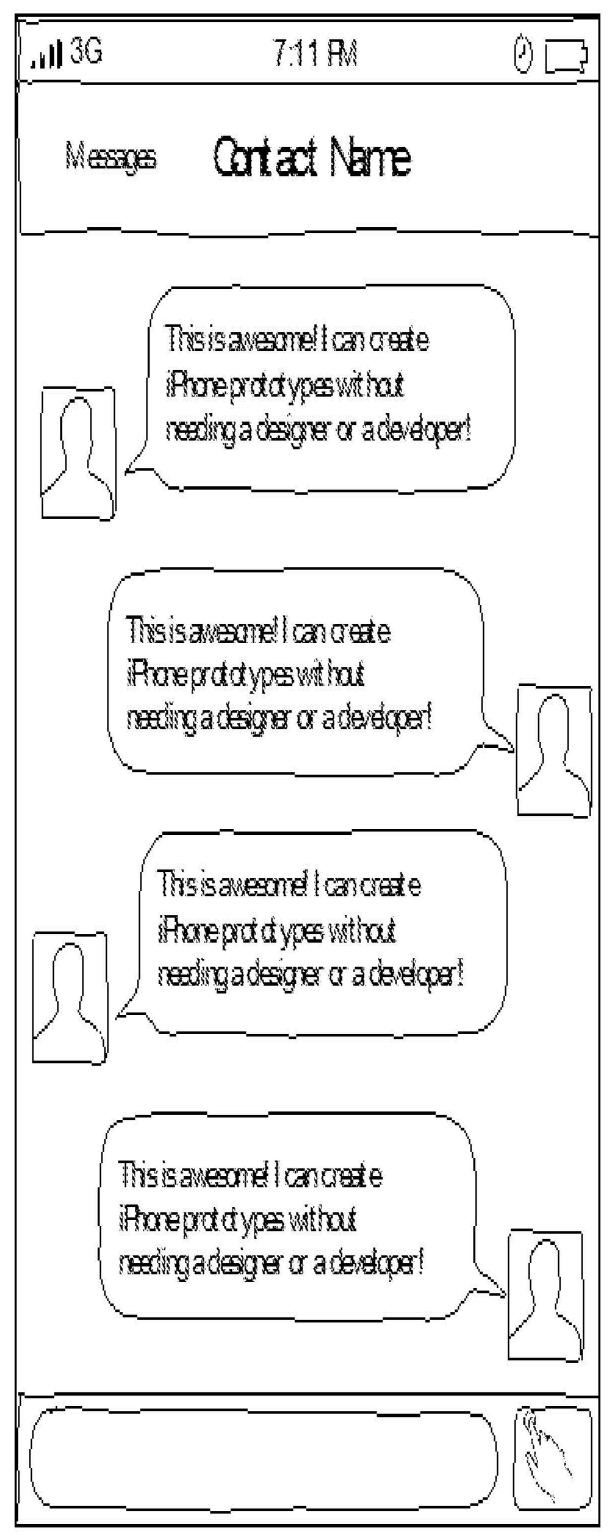

$F \mid G .1$ 


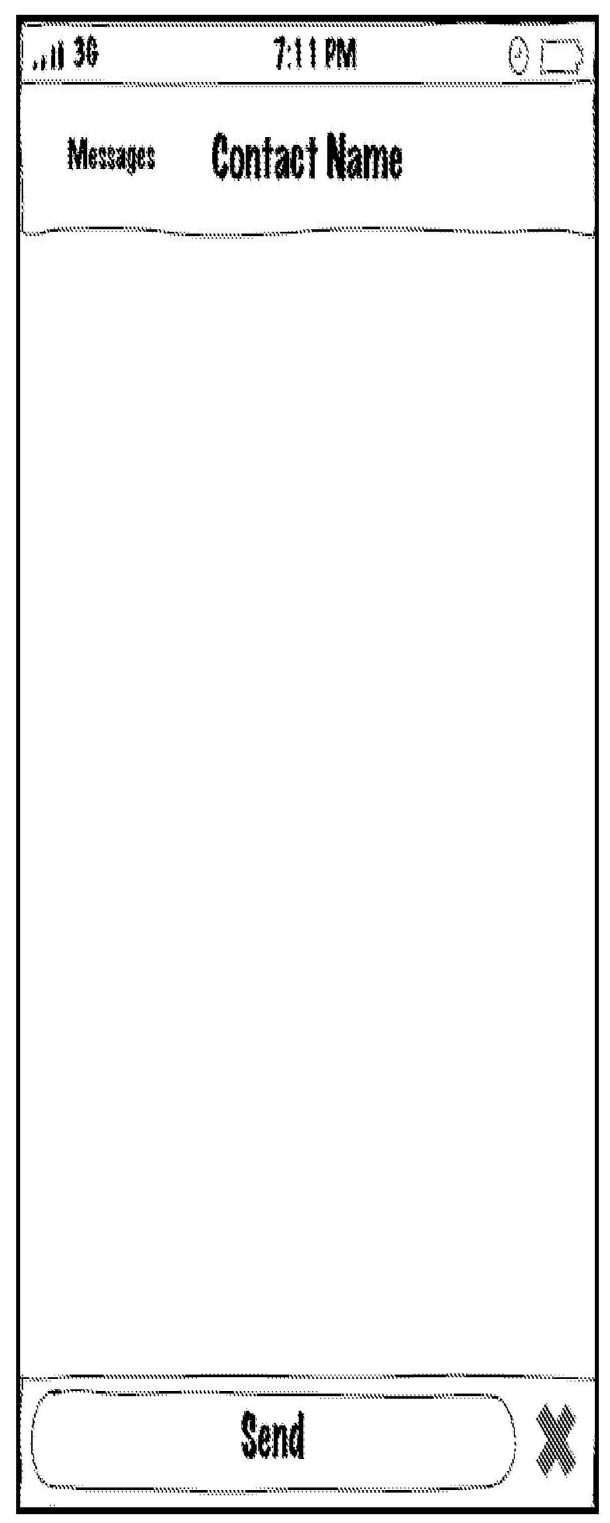

$F \mid G .2$ 

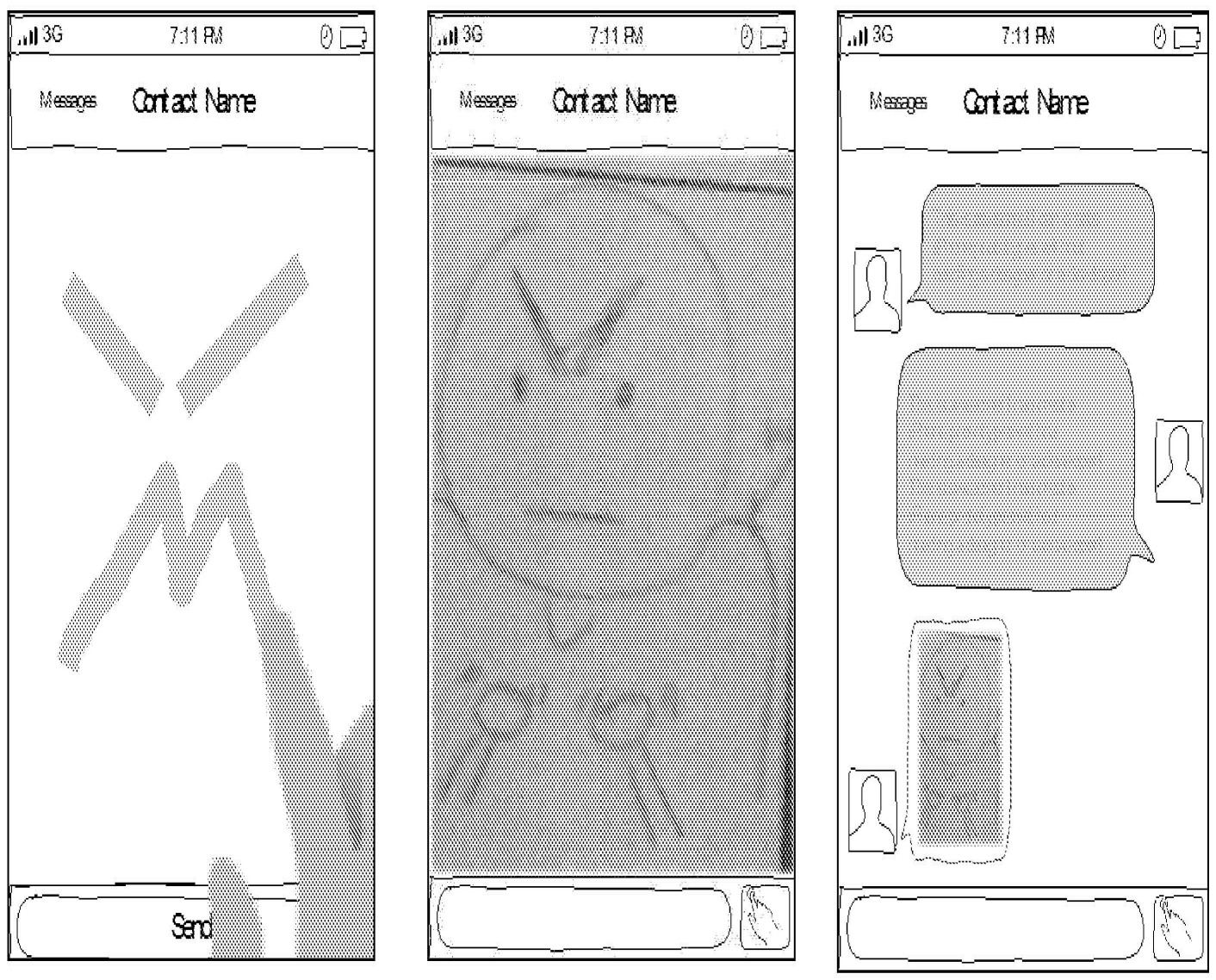

FIG. 3 

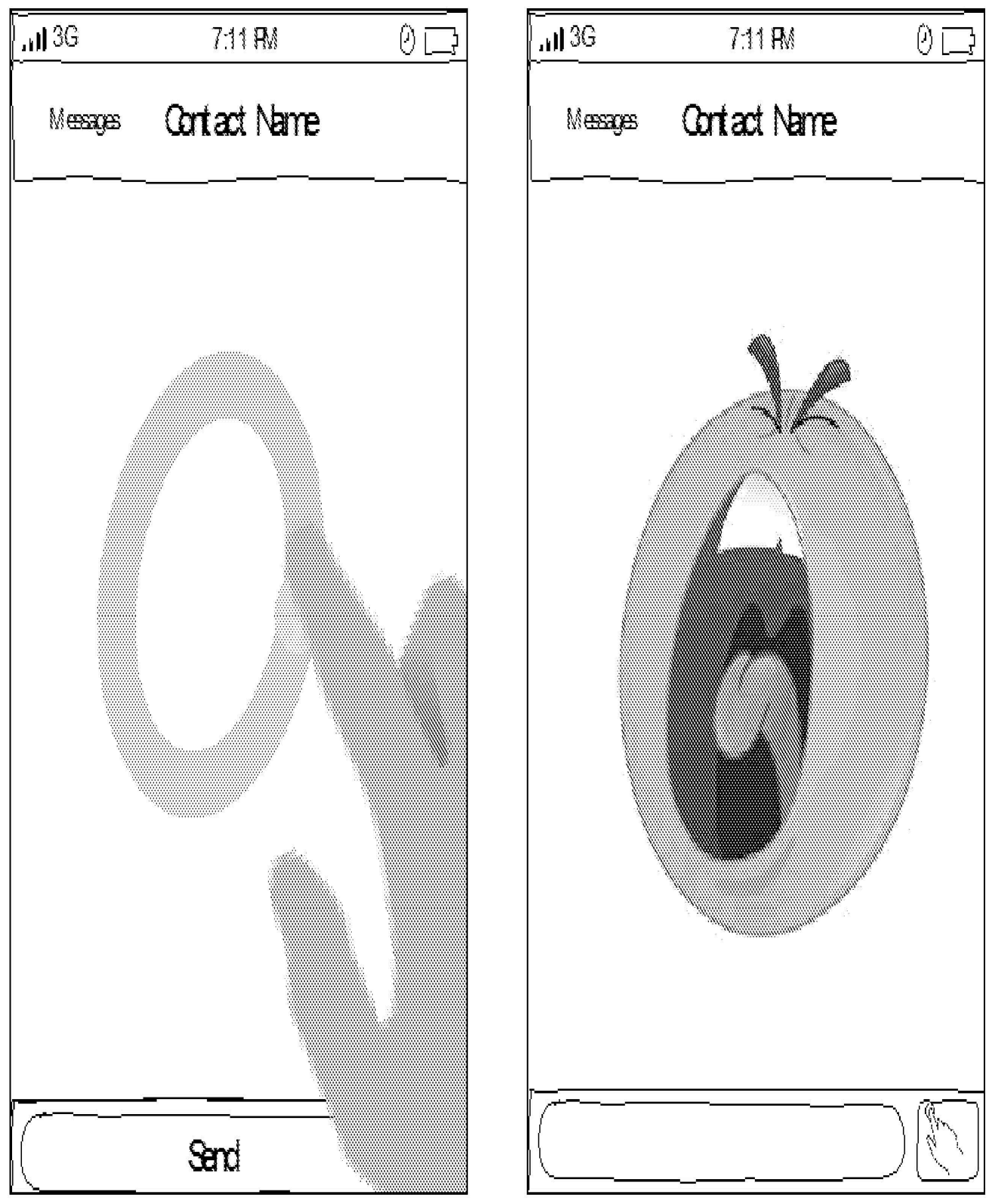

FIG. 4 

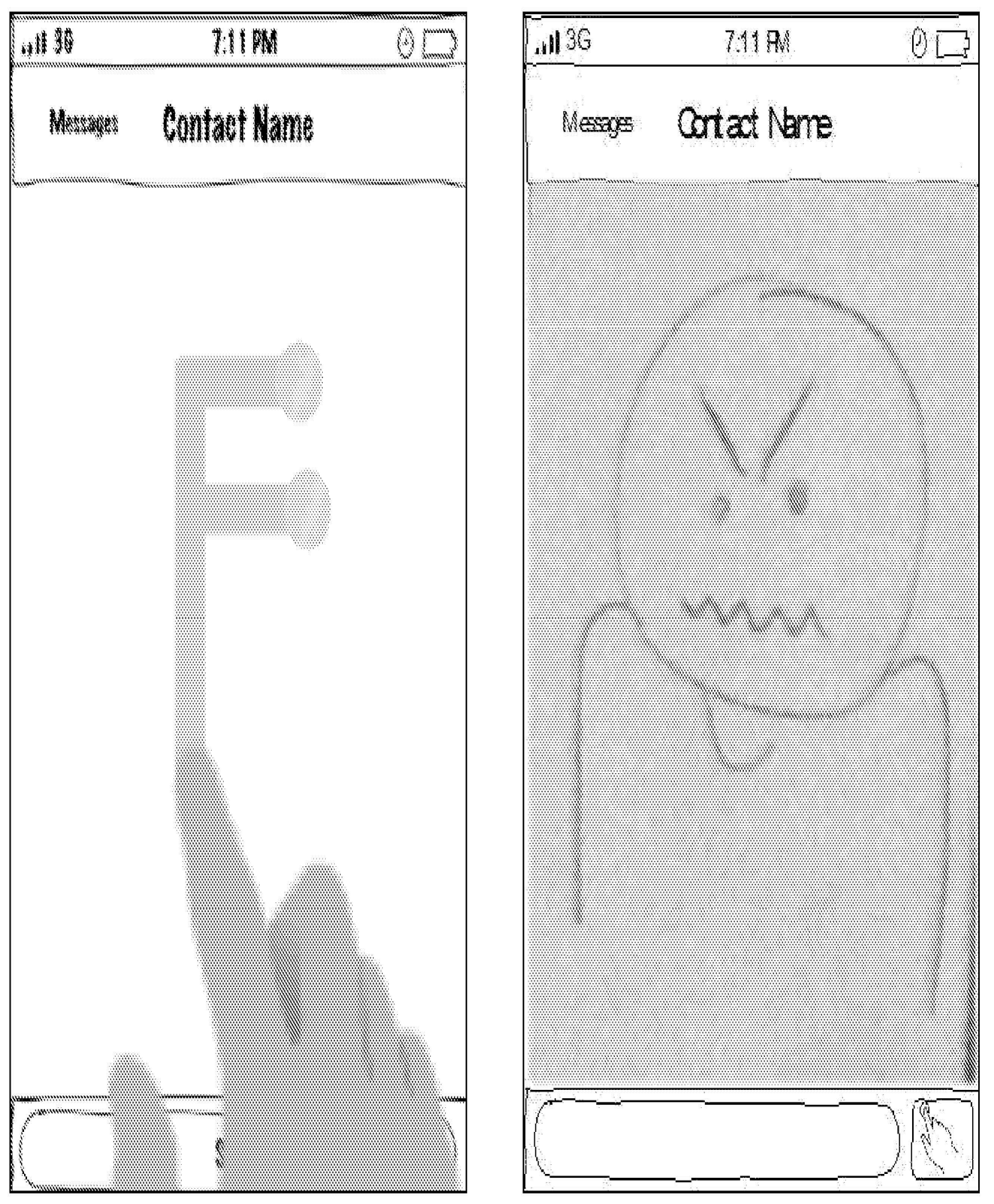

FIG. 5 

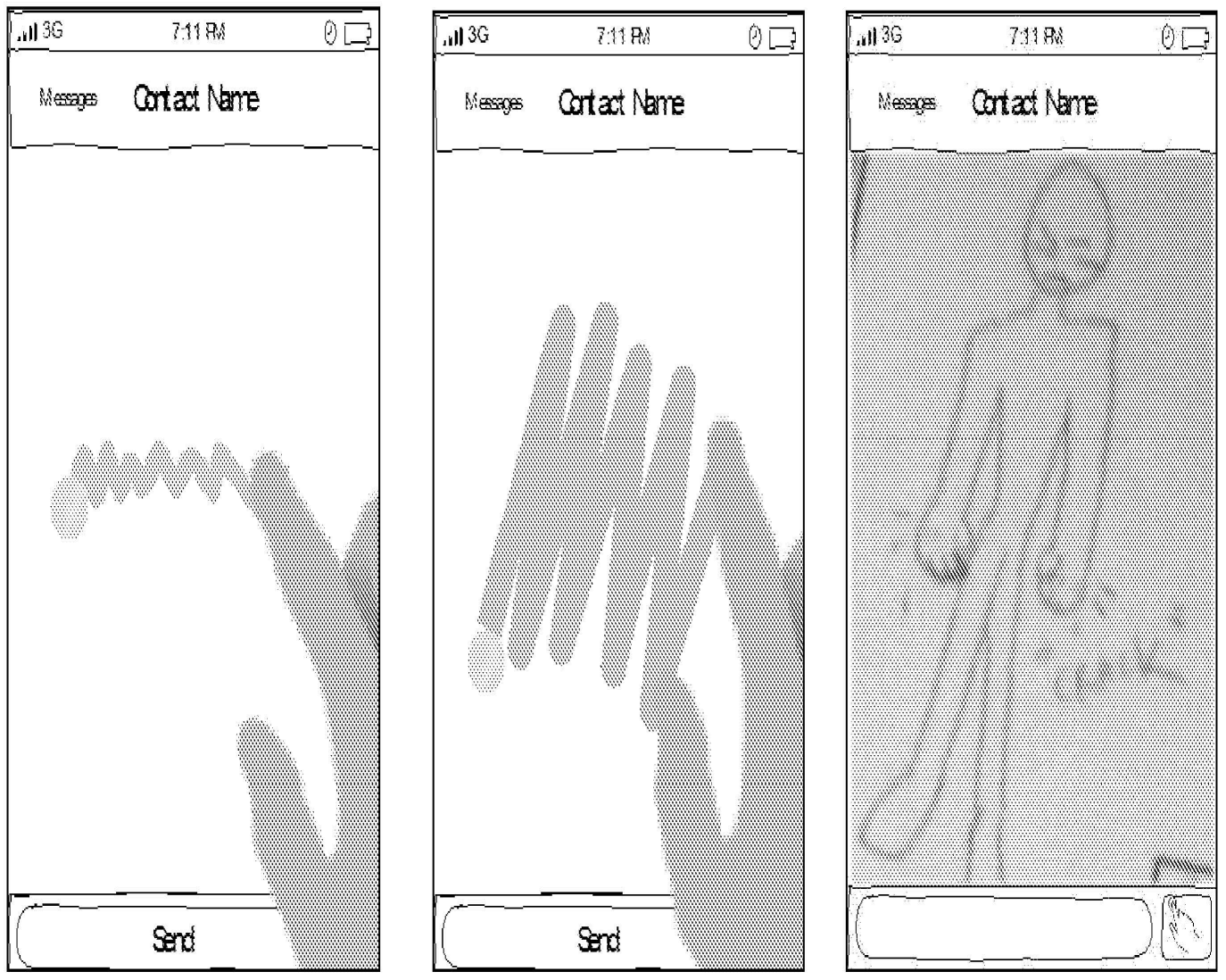

FIG. 6 

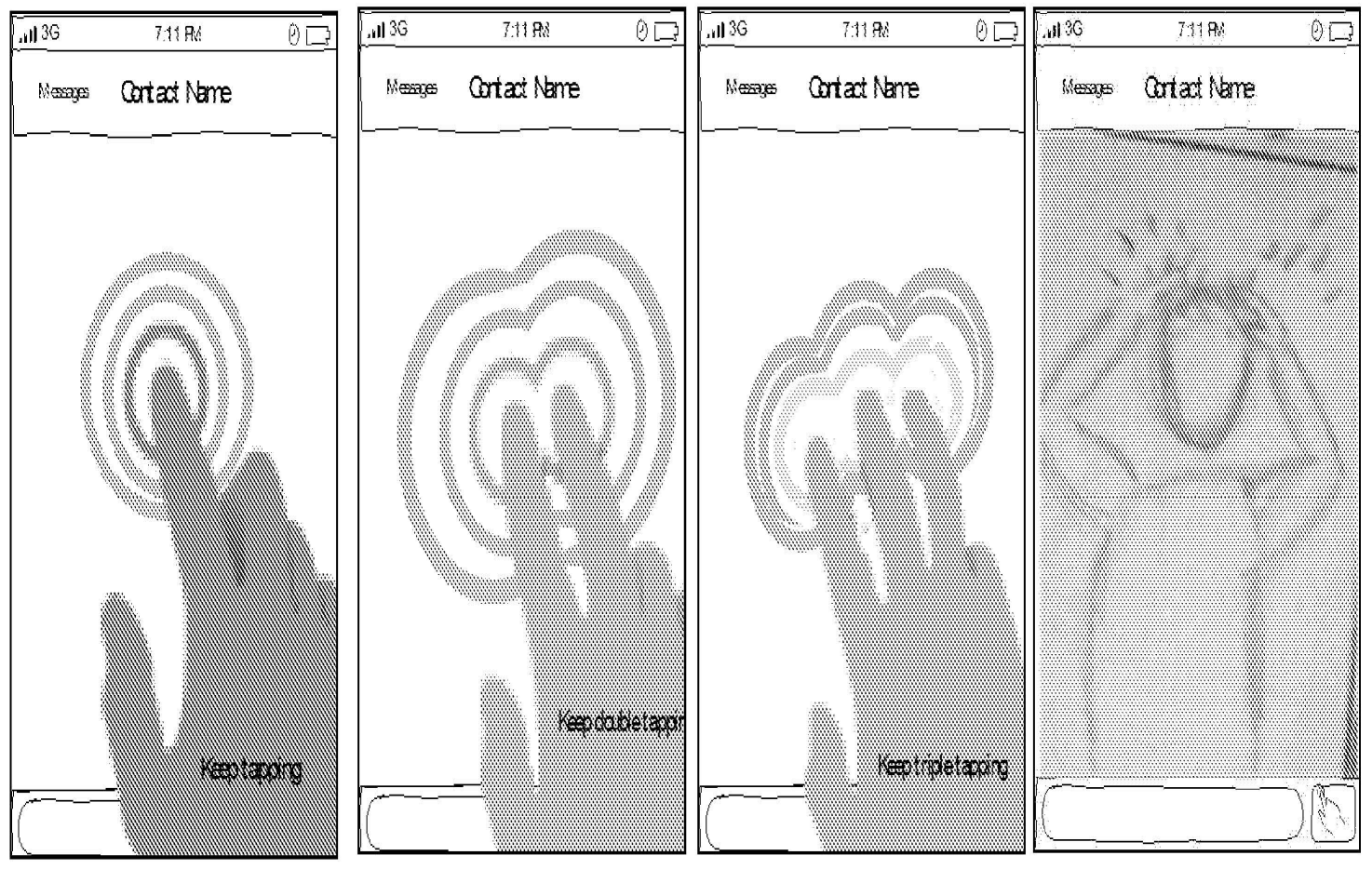

$F \mid G .7$ 

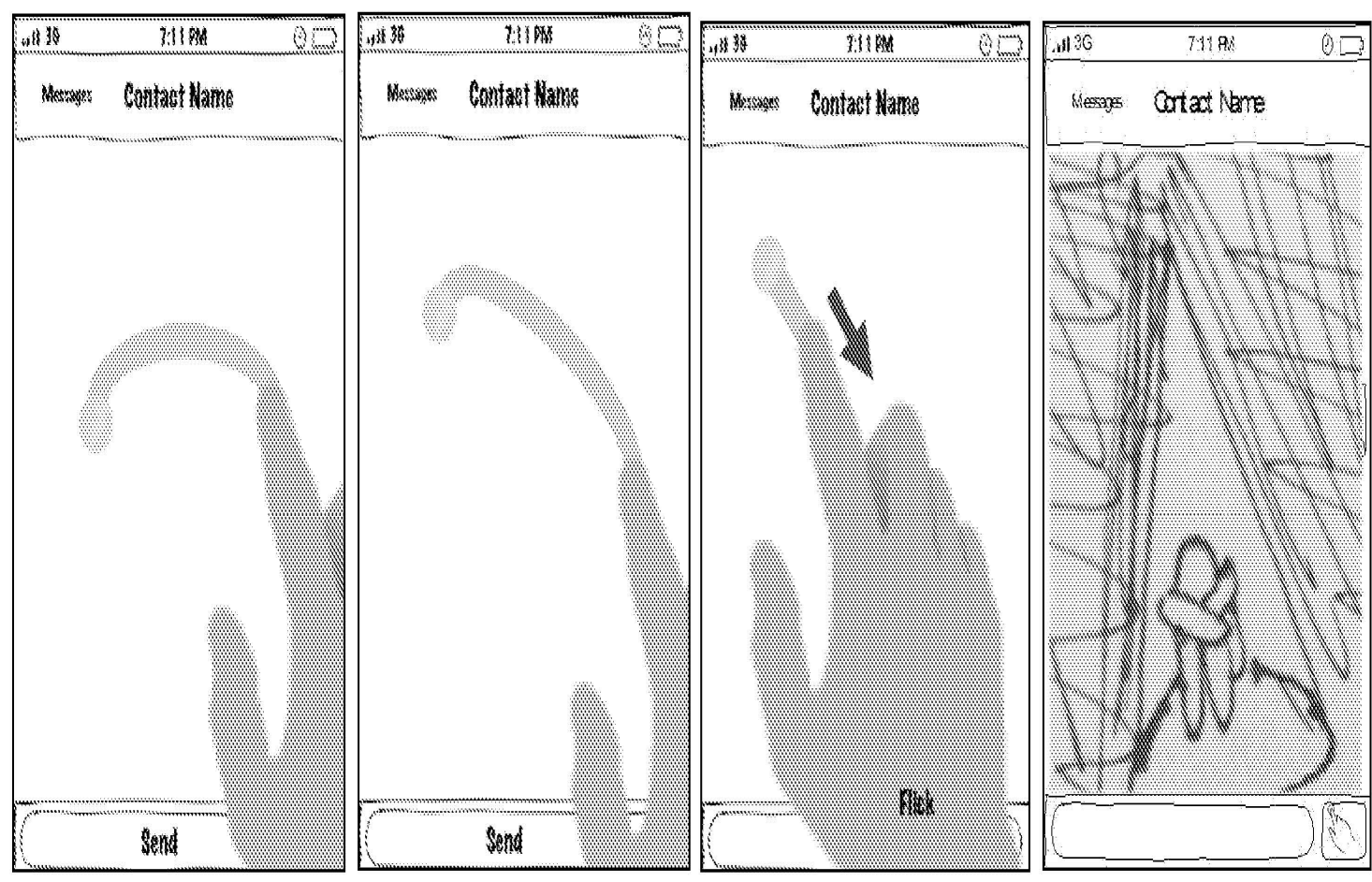

FIG. 8 

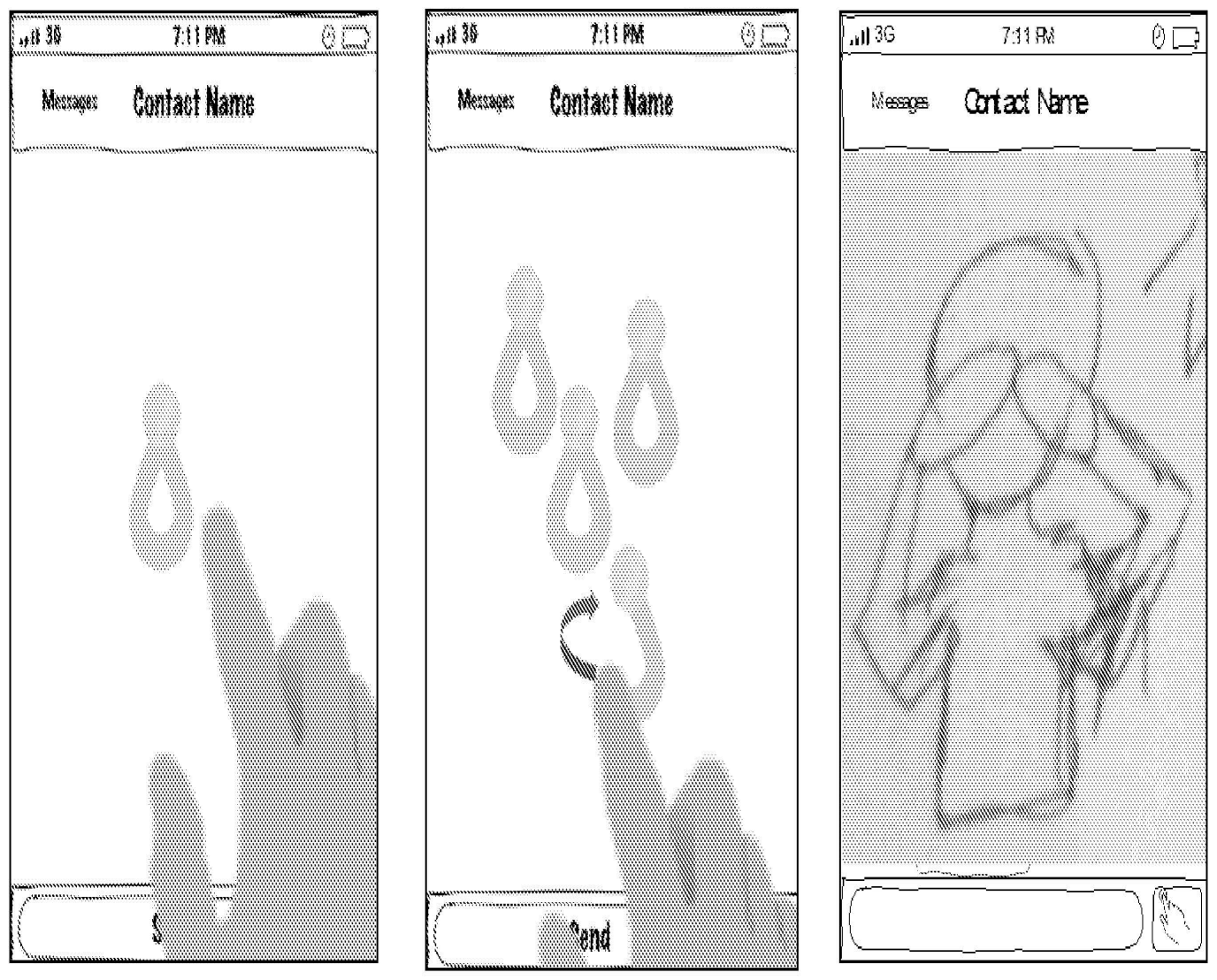

FIG. 9 

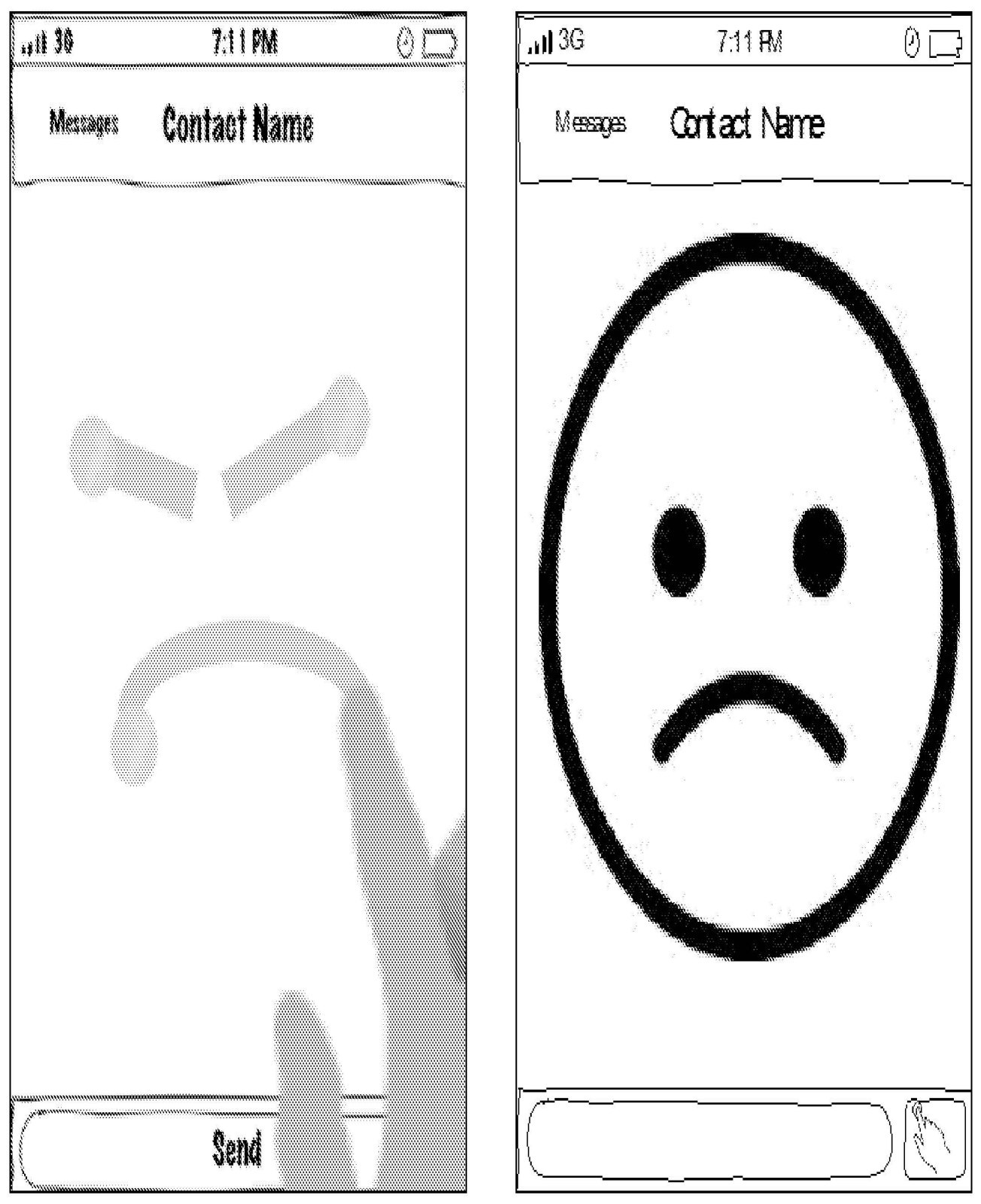

FIG. 10 


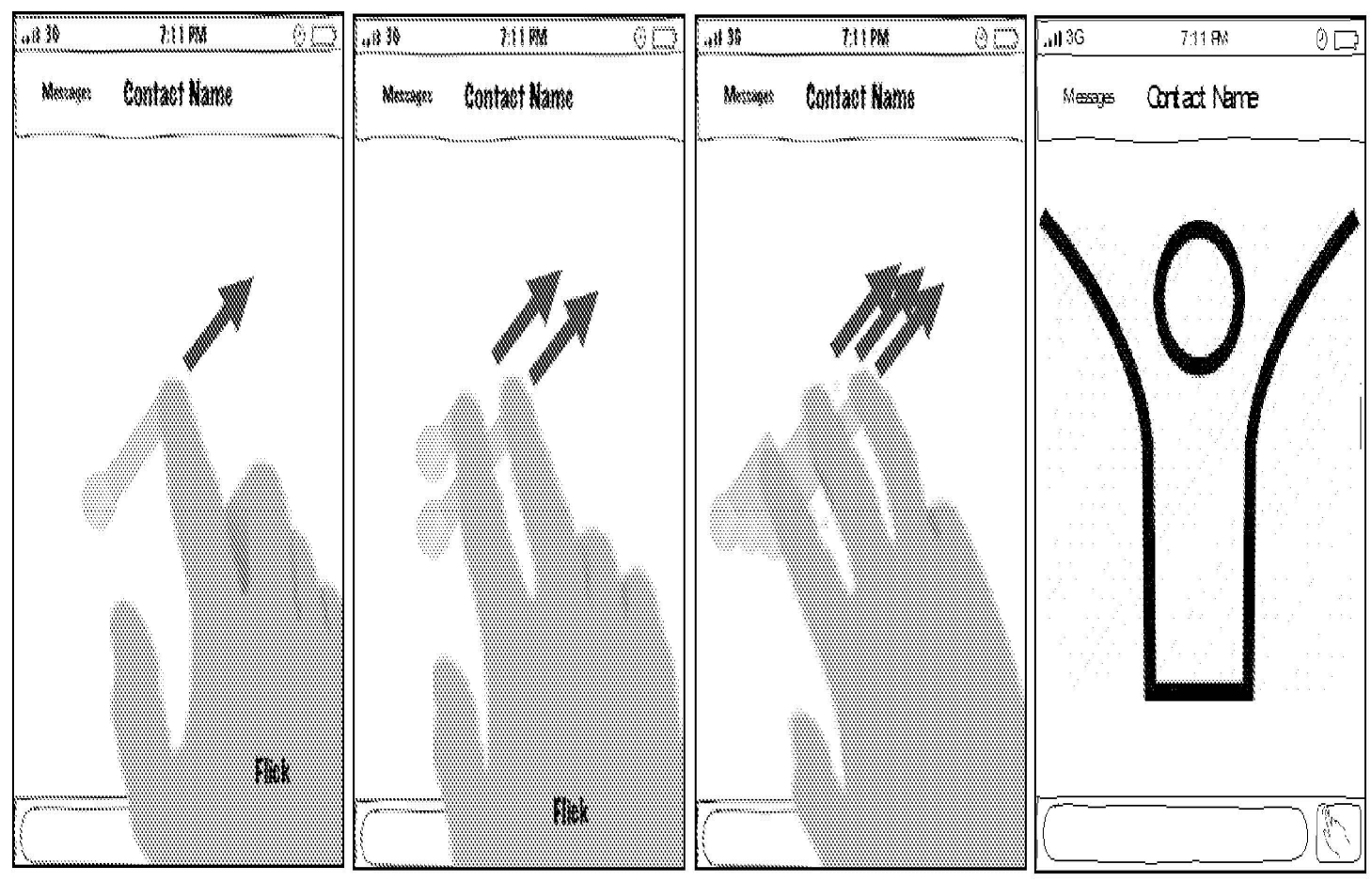

FIG. 11 

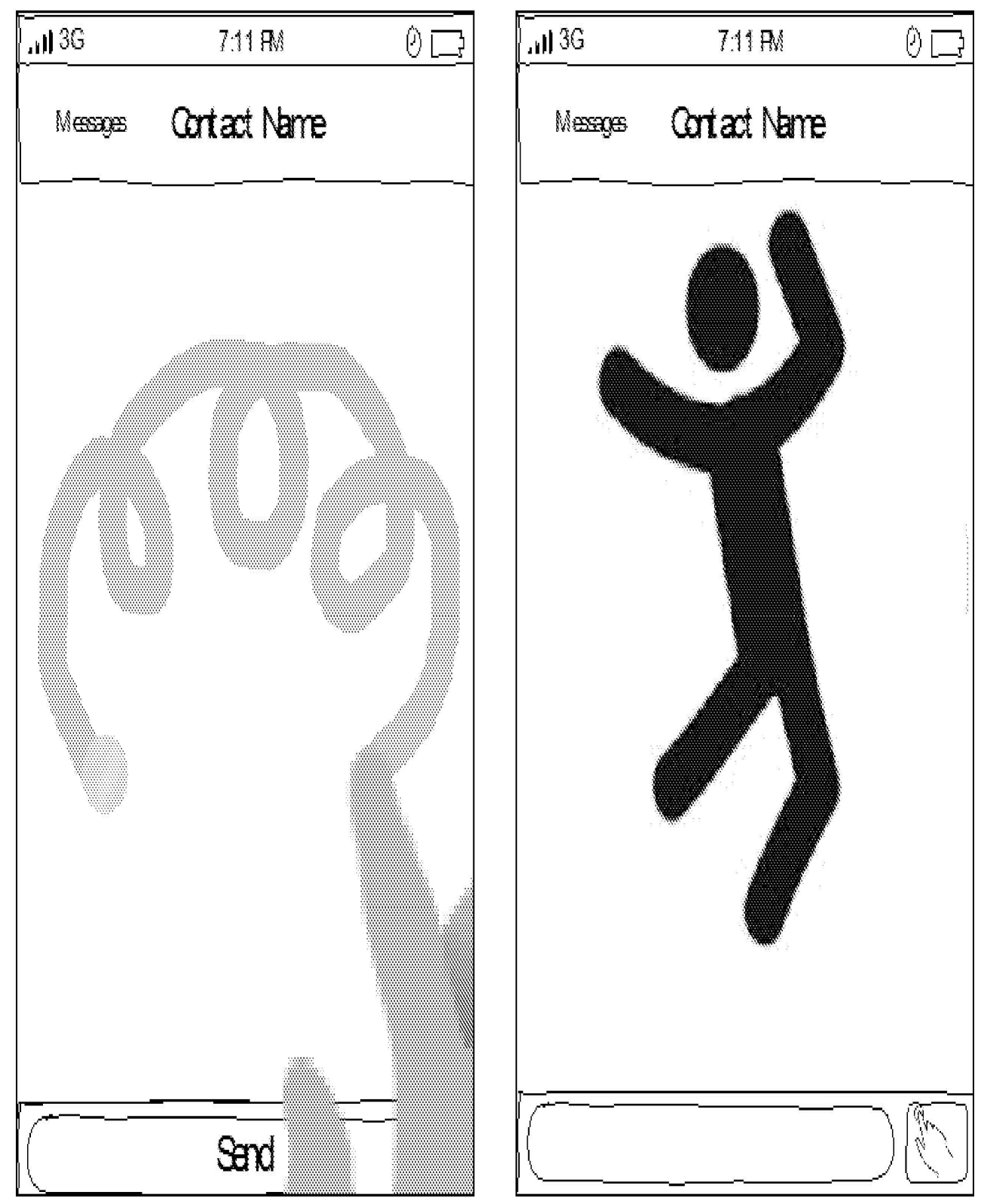

FIG. 12 


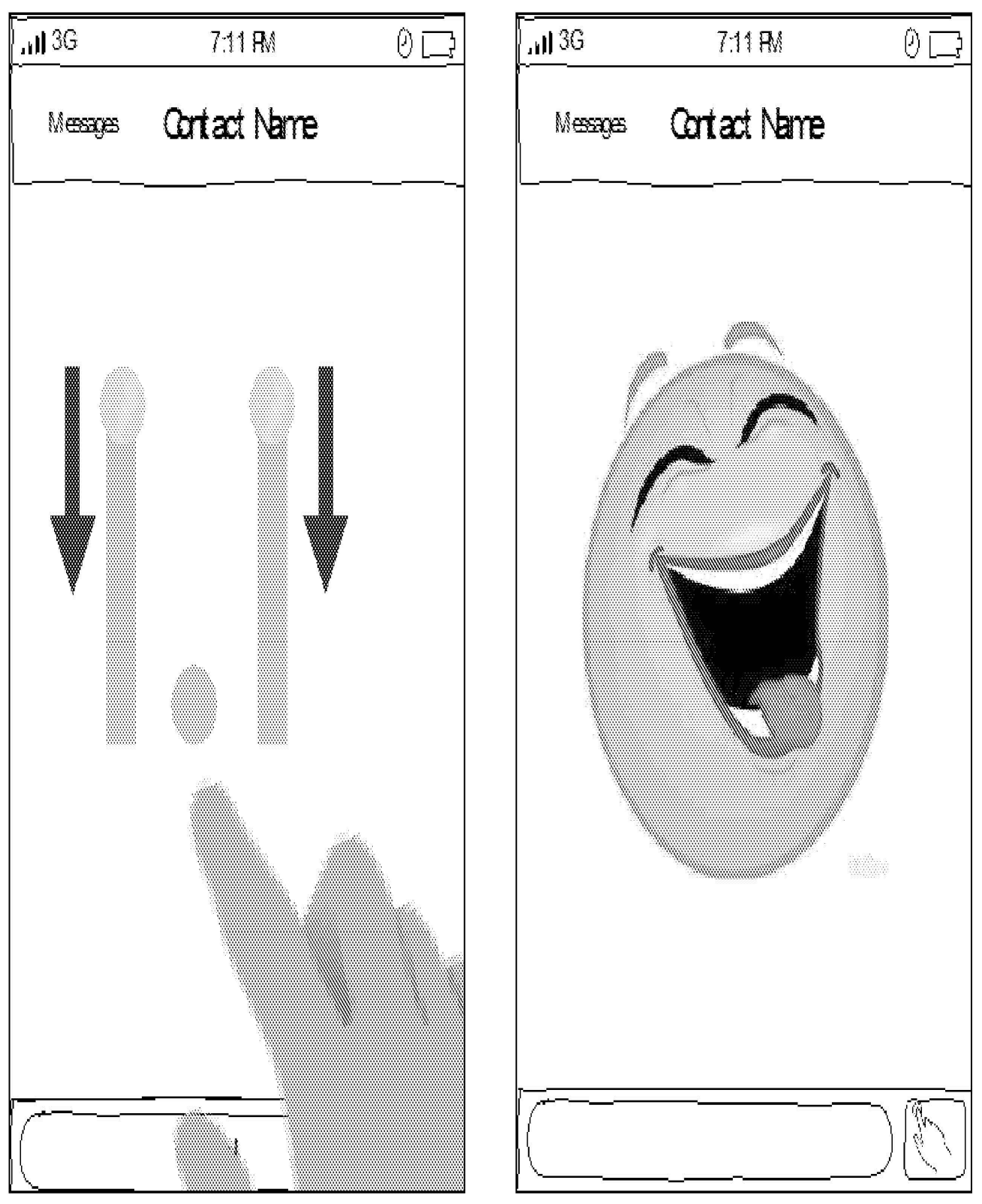

FIG. 13 

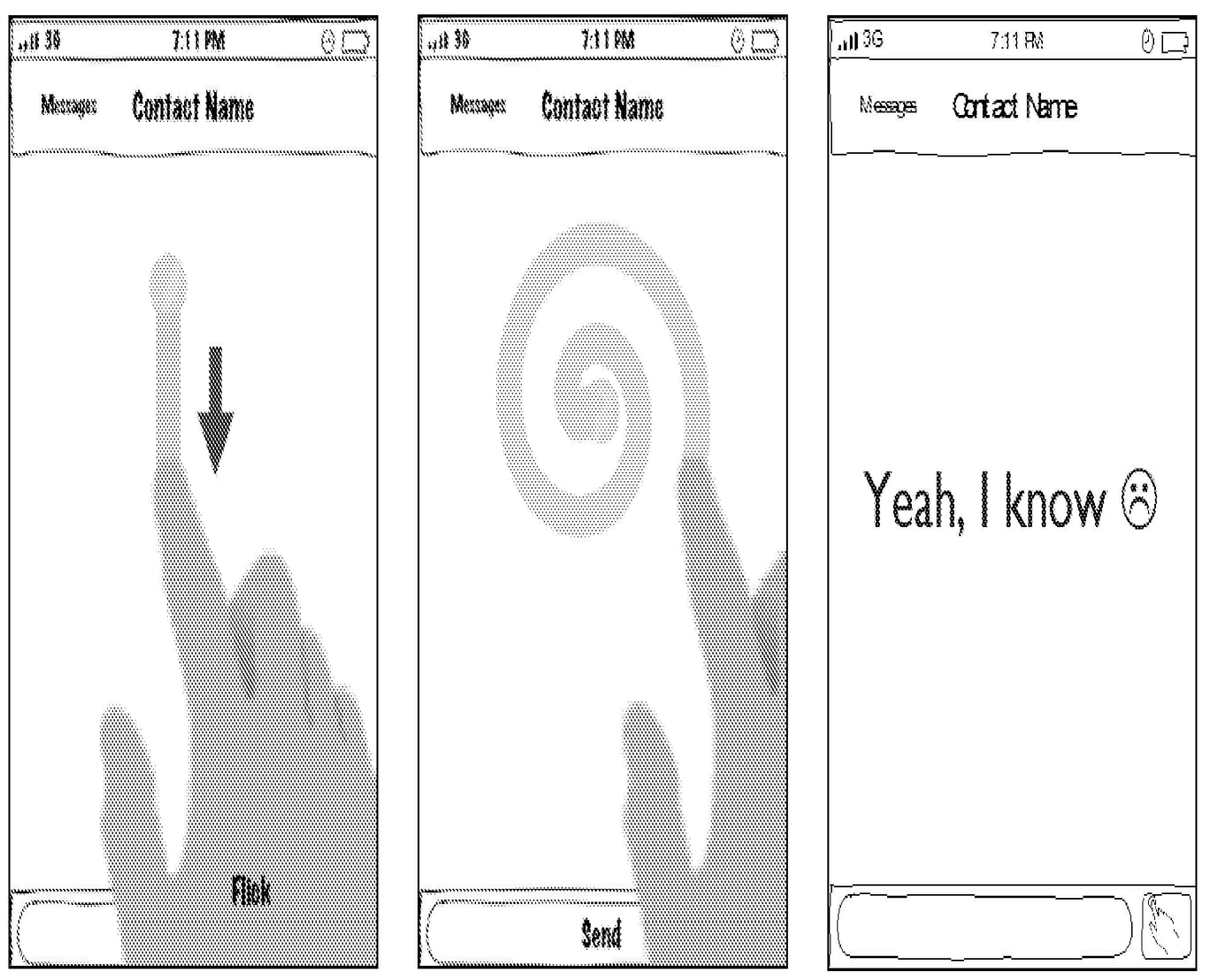

FIG. 14 

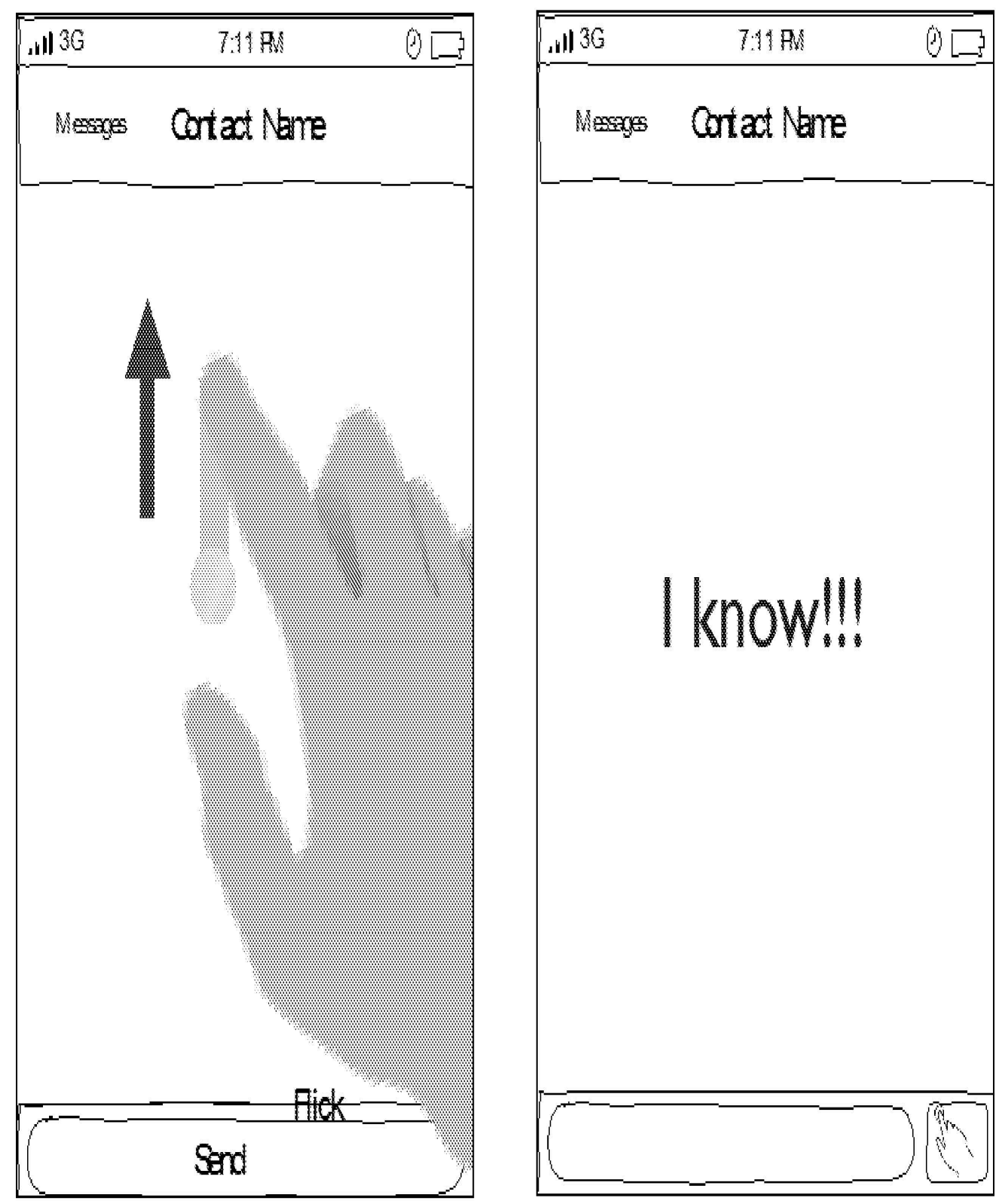

FIG. 15 

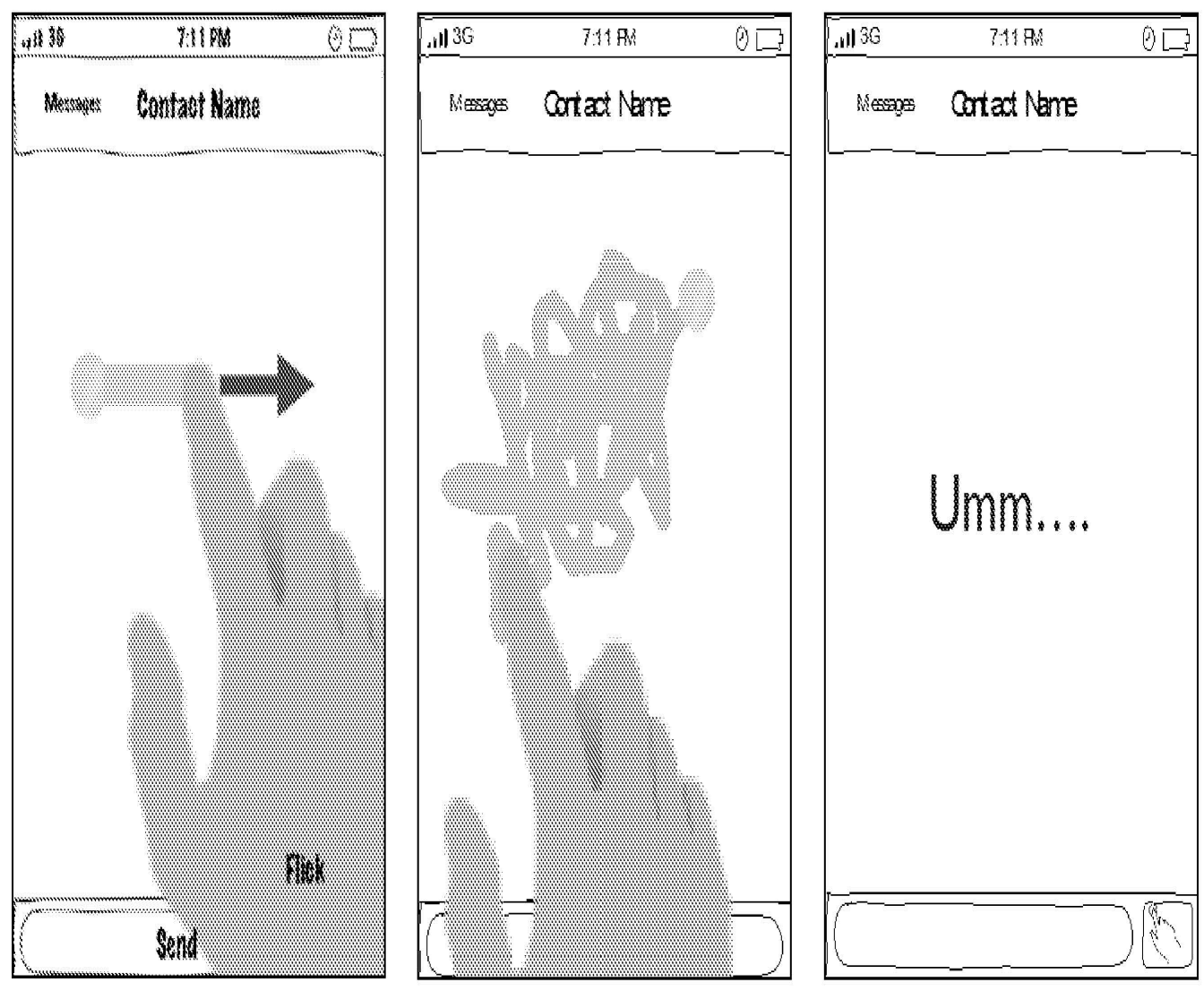

FIG. 16 

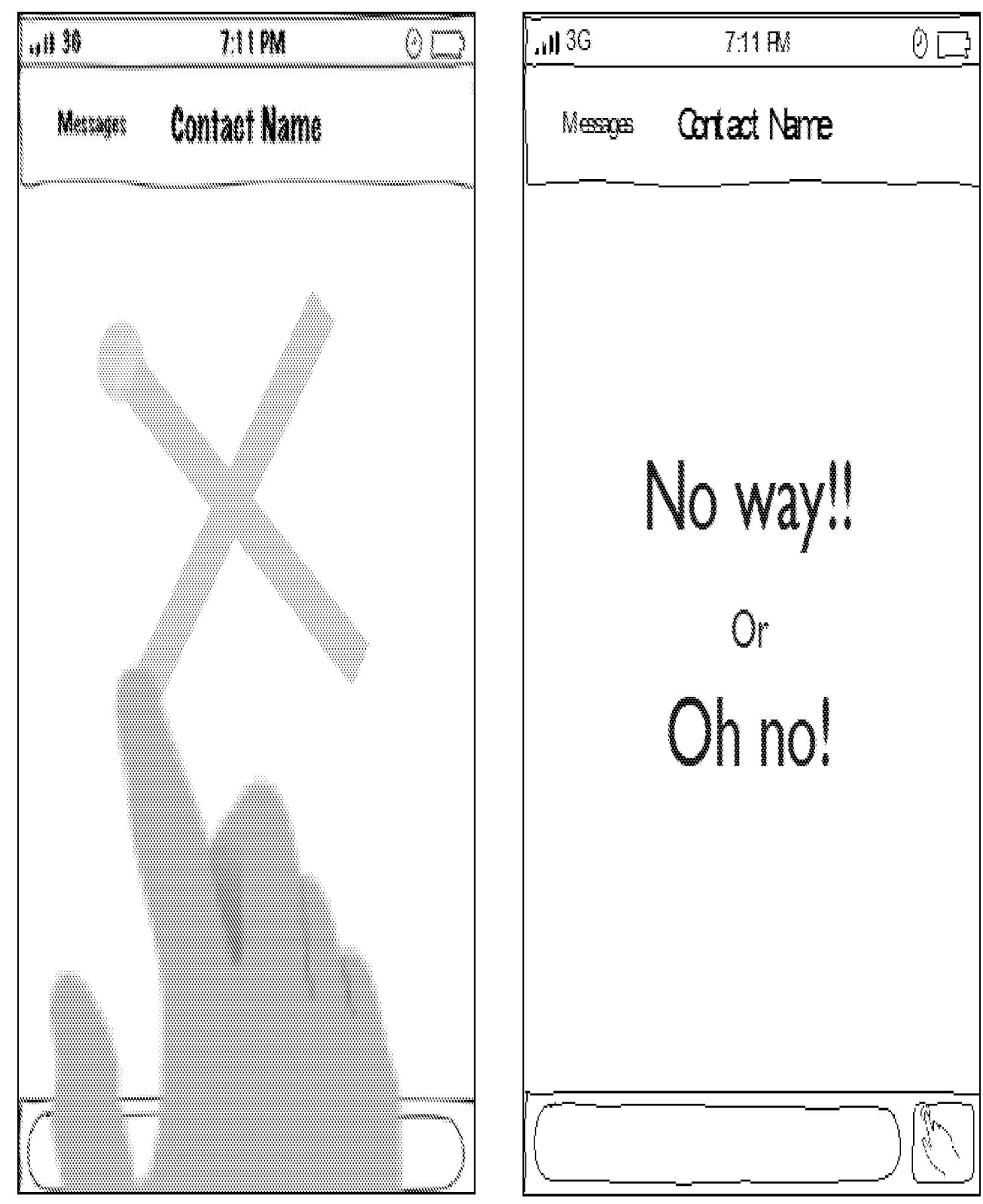

FIG. 18 

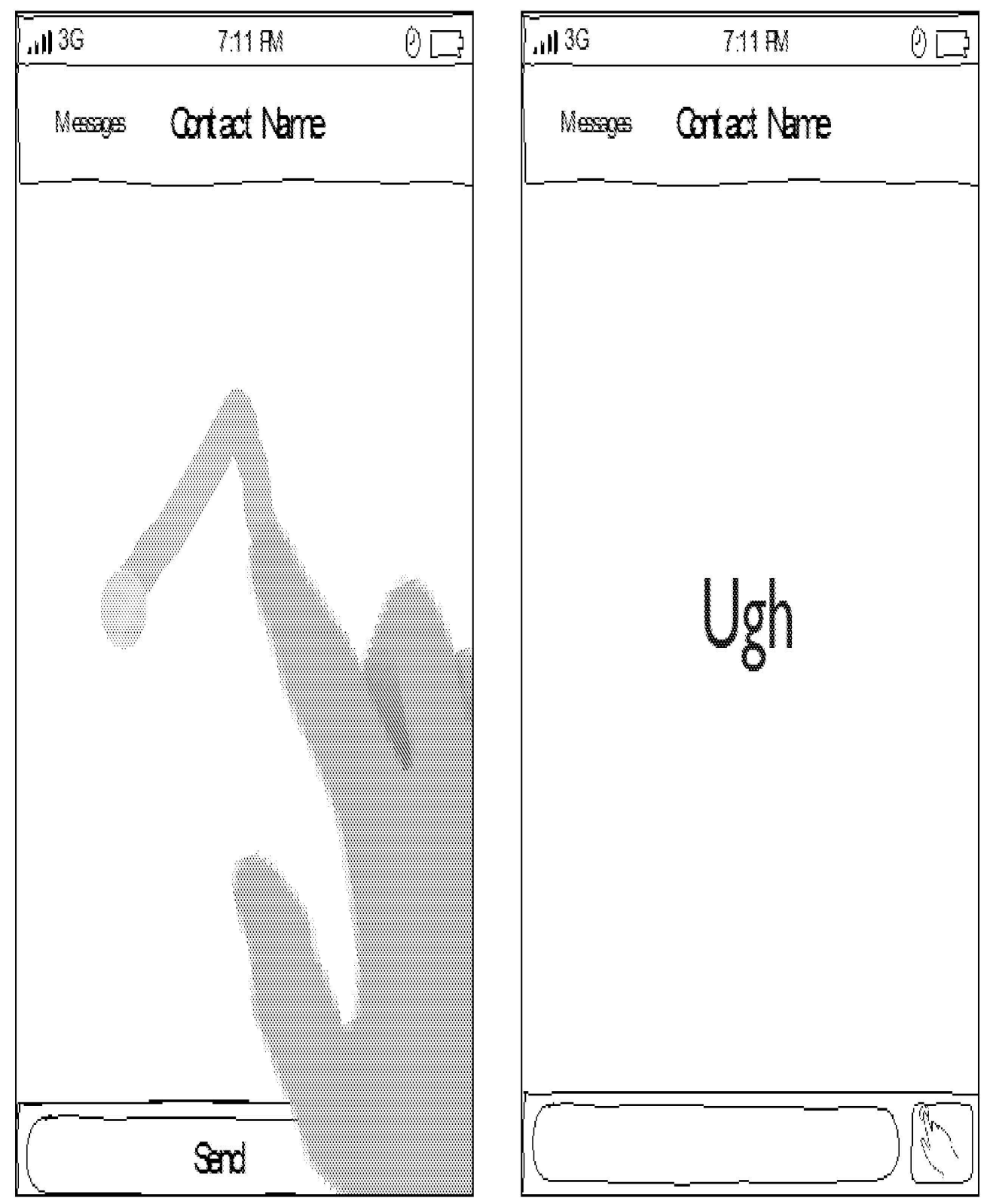

FIG. 19 

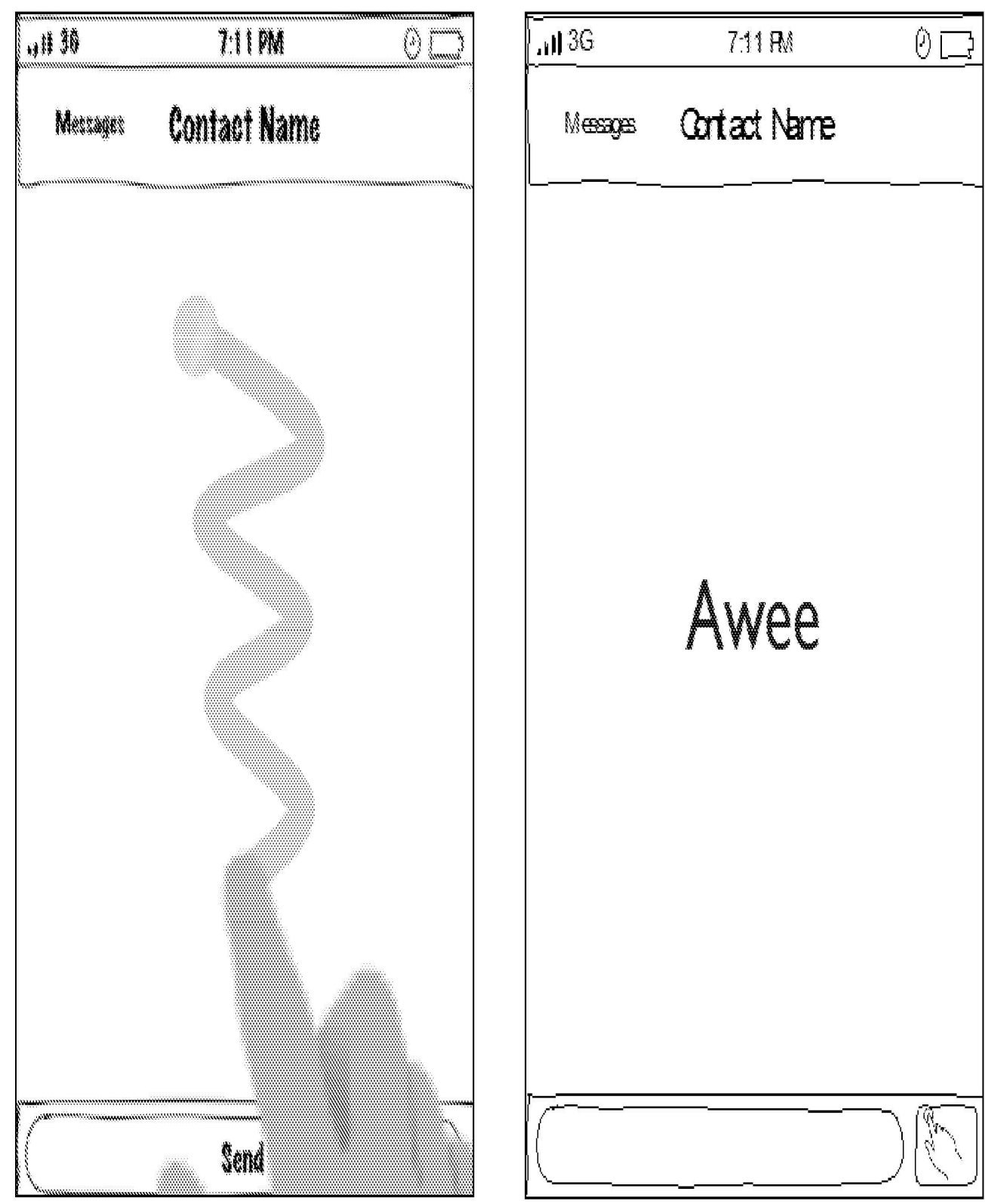

FIG. 20 

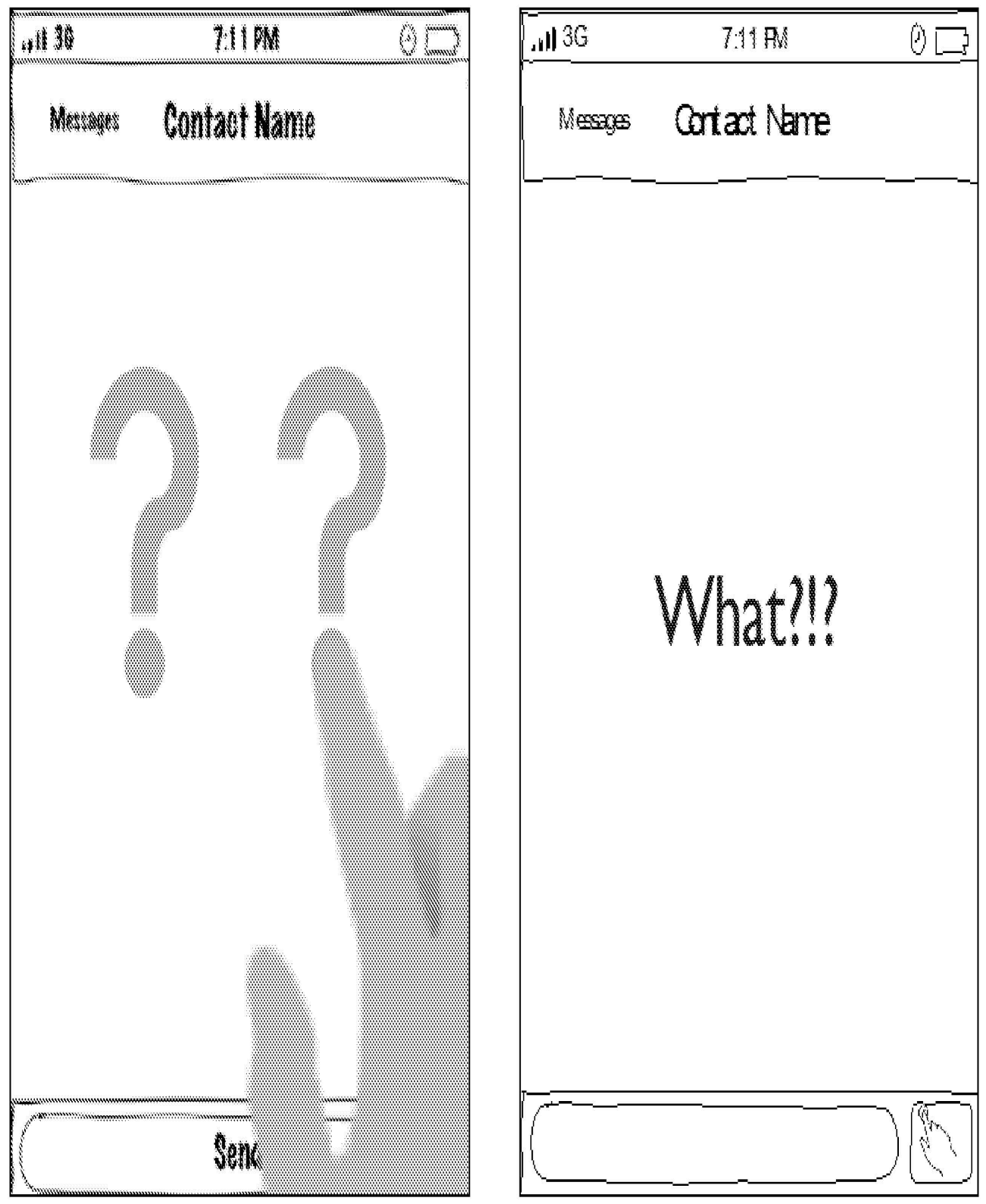

FIG. 21 


\begin{tabular}{|l|l|l|}
\hline Application Data Sheet 37 CFR 1.76 & Attorney Docket Number & IURTC-2015-124-01-US \\
\cline { 2 - 3 } & Application Number & \\
\hline Title of Invention & EMOTIONAL-GESTURE BASED COMMUNICATION METHOD \\
\hline $\begin{array}{l}\text { The application data sheet is part of the provisional or nonprovisional application for which it is being submitted. The following form contains the } \\
\text { bibliographic data arranged in a format specified by the United States Patent and Trademark Office as outlined in 37 CFR 1.76. } \\
\text { This document may be completed electronically and submitted to the Office in electronic format using the Electronic Filing System (EFS) or the } \\
\text { document may be printed and included in a paper filed application. }\end{array}$ \\
\hline
\end{tabular}

\section{Secrecy Order 37 CFR 5.2}

Portions or all of the application associated with this Application Data Sheet may fall under a Secrecy Order pursuant to 37 CFR 5.2 (Paper filers only. Applications that fall under Secrecy Order may not be filed electronically.)

\section{Inventor Information:}

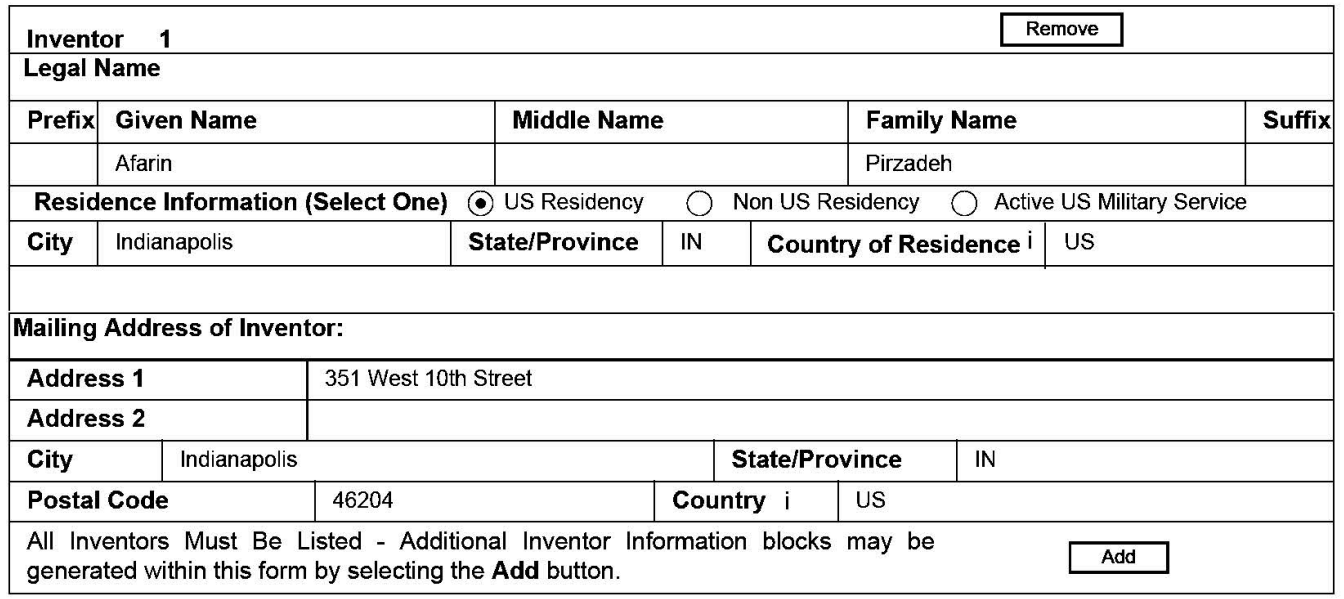

\section{Correspondence Information:}

Enter either Customer Number or complete the Correspondence Information section below. For further information see 37 CFR 1.33(a).

\begin{tabular}{|l|l|l|}
\hline \multicolumn{1}{|c|}{ An Address is being provided for the correspondence Information of this application. } \\
\hline Customer Number & 43556 & Add Email \\
\hline Email Address & inteas@faegrebd_com & Remove Email \\
\hline
\end{tabular}

\section{Application Information:}

\begin{tabular}{|l|l|l|l|}
\hline Title of the Invention & \multicolumn{4}{|l|}{ EMOTIONAL-GESTURE BASED COMMUNICATION METHOD } \\
\hline Attorney Docket Number & IURTC-2015-124-01-US & Small Entity Status Claimed & 区 \\
\hline Application Type & Provisional & Suggested Figure for Publication (if any) & \\
\hline Subject Matter & Utility & 21 & \\
\hline
\end{tabular}



Under the Paperwork Reduction Act of 1995, no persons are required to respond to a collection of information unless it contains a valid OMB control number.

\begin{tabular}{|l|l|l|l|}
\hline \multicolumn{2}{|l|}{ Application Data Sheet 37 CFR 1.76 } & Attorney Docket Number & IURTC-2015-124-01-US \\
\cline { 3 - 4 } & Application Number & \\
\hline Title of Invention & EMOTIONAL-GESTURE BASED COMMUNICATION METHOD \\
\hline
\end{tabular}

\section{Publication Information:}

Request Early Publication (Fee required at time of Request 37 CFR 1.219)

Request Not to Publish. I hereby request that the attached application not be published under 35 U.S.C. 122(b) and certify that the invention disclosed in the attached application has not and will not be the subject of an application filed in another country, or under a multilateral international agreement, that requires publication at eighteen months after filing.

\section{Representative Information:}

\begin{tabular}{|c|c|c|c|}
\hline \multicolumn{4}{|c|}{$\begin{array}{l}\text { Representative information should be provided for all practitioners hav } \\
\text { this information in the Application Data Sheet does not constitute a power } \\
\text { Either enter Customer Number or complete the Representative Name sec } \\
\text { Number will be used for the Representative Information during processing. }\end{array}$} \\
\hline Please Select One: & Customer Number & US Patent Practitioner & Limited Recognition (37 CFR 11.9) \\
\hline Customer Number & \multicolumn{3}{|l|}{43556} \\
\hline
\end{tabular}

\section{Domestic Benefit/National Stage Information:}

\begin{tabular}{|c|c|c|c|}
\hline \multicolumn{4}{|c|}{$\begin{array}{l}\text { This section allows for the applicant to either claim benefit under } 35 \text { U.S.C. } 119(\mathrm{e}), 120,121 \text {, or } 365 \text { (c) or indicate } \\
\text { National Stage entry from a PCT application. Providing this information in the application data sheet constitutes the } \\
\text { specific reference required by } 35 \text { U.S.C. } 119(\text { e) or } 120 \text {, and } 37 \text { CFR } 1.78 \text {. }\end{array}$} \\
\hline Prior Application Status & & \multicolumn{2}{|r|}{\begin{tabular}{|l|} 
Remove \\
\end{tabular}} \\
\hline Application Number & Continuity Type & Prior Application Number & Filing Date (YYYY-MM-DD) \\
\hline $\begin{array}{l}\text { dditional Domestic Ben } \\
\text { / selecting the Add but }\end{array}$ & age & atec & Add \\
\hline
\end{tabular}

\section{Foreign Priority Information:}

This section allows for the applicant to claim priority to a foreign application. Providing this information in the application data sheet constitutes the claim for priority as required by 35 U.S.C. $119(\mathrm{~b})$ and 37 CFR 1.55 (d). When priority is claimed to a foreign application that is eligible for retrieval under the priority document exchange program (PDX) ithe information will be used by the Office to automatically attempt retrieval pursuant to 37 CFR 1.55(h)(1) and (2). Under the PDX program, applicant bears the ultimate responsibility for ensuring that a copy of the foreign application is received by the Office from the participating foreign intellectual property office, or a certified copy of the foreign priority application is filed, within the time period specified in $37 \mathrm{CFR} 1.55(\mathrm{~g})(1)$

\begin{tabular}{|l|l|l|c|}
\hline \multicolumn{1}{|c|}{ Remove } \\
\hline \multicolumn{1}{|c|}{ Application Number } & Country i & Filing Date (YYYY-MM-DD) & Access Code' (if applicable) \\
\hline $\begin{array}{l}\text { Additional Foreign Priority Data may be generated within this form by selecting the } \\
\text { Add button. }\end{array}$
\end{tabular}

EFS Web 2.2.6 


\section{Statement under 37 CFR 1.55 or 1.78 for AIA (First Inventor to File) Transition Applications}

This application (1) claims priority to or the benefit of an application filed before March 16, 2013 and (2) also

contains, or contained at any time, a claim to a claimed invention that has an effective filing date on or after March 16, 2013.

\section{Authorization to Permit Access:}

A Authorization to Permit Access to the Instant Application by the Participating Offices

If checked, the undersigned hereby grants the USPTO authority to provide the European Patent Office (EPO),

the Japan Patent Office (JPO), the Korean Intellectual Property Office (KIPO), the World Intellectual Property Office (WIPO)

and any other intellectual property offices in which a foreign application claiming priority to the instant patent application

is filed access to the instant patent application. See 37 CFR 1.14(c) and (h). This box should not be checked if the applicant

does not wish the EPO, JPO, KIPO, WIPO, or other intellectual property office in which a foreign application claiming priority

to the instant patent application is filed to have access to the instant patent application

In accordance with 37 CFR 1.14(h)(3), access will be provided to a copy of the instant patent application with respect

to: 1) the instant patent application-as-filed; 2) any foreign application to which the instant patent application

claims priority under 35 U.S.C. 119 (a)-(d) if a copy of the foreign application that satisfies the certified copy requirement of

37 CFR 1.55 has been filed in the instant patent application; and 3) any U.S. application-as-filed from which benefit is

sought in the instant patent application

In accordance with $37 \mathrm{CFR} 1.14$ (c), access may be provided to information concerning the date of filing this Authorization

\section{Applicant Information:}

Providing assignment information in this section does not substitute for compliance with any requirement of part 3 of Title 37 of CFR

to have an assignment recorded by the Office. 

Under the Paperwork Reduction Act of 1995, no persons are required to respond to a collection of information unless it contains a valid OMB control number.

\section{Application Data Sheet 37 CFR 1.76}

Attorney Docket Number

Application Number

Title of Invention EMOTIONAL-GESTURE BASED COMMUNICATION METHOD

Applicant 1

If the applicant is the inventor (or the remaining joint inventor or inventors under 37 CFR 1.45), this section should not be completed The information to be provided in this section is the name and address of the legal representative who is the applicant under $37 \mathrm{CFR}$

1.43; or the name and address of the assignee, person to whom the inventor is under an obligation to assign the invention, or person who otherwise shows sufficient proprietary interest in the matter who is the applicant under 37 CFR 1.46. If the applicant is an

applicant under 37 CFR 1.46 (assignee, person to whom the inventor is obligated to assign, or person who otherwise shows sufficient proprietary interest) together with one or more joint inventors, then the joint inventor or inventors who are also the applicant should be identified in this section.

$$
\text { Clear }
$$

\begin{tabular}{|c|c|c|c|}
\hline \multicolumn{3}{|c|}{ Legal Representative under 35 U.S.C. 117} & Joint Inventor \\
\hline \multicolumn{2}{|c|}{ Person to whom the inventor is obligated to assign. } & \multicolumn{2}{|c|}{ Person who shows sufficient proprietary interest } \\
\hline \multicolumn{4}{|c|}{ If applicant is the legal representative, indicate the authority to file the patent application, the inventor is: } \\
\hline \multicolumn{4}{|c|}{ Name of the Deceased or Legally Incapacitated Inventor: } \\
\hline \multicolumn{4}{|c|}{ If the Applicant is an Organization check here. $\mathrm{X}$} \\
\hline Organization Name & \multicolumn{3}{|c|}{ Indiana University Research and Technology Corp. } \\
\hline \multicolumn{4}{|c|}{ Mailing Address Information: } \\
\hline Address 1 & \multicolumn{3}{|l|}{351 West 10th Street } \\
\hline \multicolumn{4}{|l|}{ Address 2} \\
\hline City & Indianapolis & State/Province & IN \\
\hline \multicolumn{2}{|l|}{\begin{tabular}{l|l} 
Country i & US \\
\end{tabular}} & Postal Code & 46202 \\
\hline Phone Number & & Fax Number & \\
\hline \multicolumn{4}{|l|}{ Email Address } \\
\hline Additional Applicant & $y$ be generated within & cting the Ad & Add \\
\hline
\end{tabular}

\section{Non-Applicant Assignee Information:}

Providing assignment information in this section does not subsitute for compliance with any requirement of part 3 of Title 37 of CFR to have an assignment recorded by the Office

\section{Assignee 1}

Complete this section only if non-applicant assignee information is desired to be included on the patent application publication in accordance with 37 CFR 1.215(b). Do not include in this section an applicant under 37 CFR 1.46 (assignee, person to whom the inventor is obligated to assign, or person who otherwise shows sufficient proprietary interest), as the patent application publication will include the name of the applicant(s)

\begin{tabular}{|l|l|}
\hline & Remove \\
\hline If the Assignee is an Organization check here.
\end{tabular}

EFS Web 2.2.6 

Under the Paperwork Reduction Act of 1995 , no persons are required to respond to a collection of information unless it contains a valid OMB control number.

\begin{tabular}{|l|l|l|l|}
\hline \multicolumn{2}{|l|}{ Application Data Sheet 37 CFR 1.76 } & Attorney Docket Number & IURTC-2015-124-01-US \\
\cline { 3 - 4 } & Application Number & \\
\hline Title of Invention & EMOTIONAL-GESTURE BASED COMMUNICATION METHOD \\
\hline
\end{tabular}

\begin{tabular}{|l|l|l|l|l|}
\hline \hline Prefix & Given Name & Middle Name & Family Name & Suffix \\
\hline & & & & \\
\hline
\end{tabular}

Mailing Address Information:

Address 1

Address 2

\begin{tabular}{|l|l|l|l|}
\hline \multicolumn{2}{|l|}{ City } & State/Province & \\
\hline Country i & & Postal Code & \\
\hline Phone Number & Fax Number & \\
\hline Email Address & & \\
\hline Additional Assignee Data may be generated within this form by selecting the Add button. & Add \\
\hline
\end{tabular}

\begin{tabular}{ll} 
Signature: & Remove \\
\hline
\end{tabular}

NOTE: This form must be signed in accordance with 37 CFR 1.33. See 37 CFR 1.4 for signature requirements and certifications

\begin{tabular}{|c|c|c|c|c|c|}
\hline Signature & \multicolumn{3}{|c|}{ jjoel a henry/ } & Date (YYYY-MM-DD) & $2015-04-20$ \\
\hline First Name & Joel A. & Last Name & Henry & Registration Number & 72970 \\
\hline
\end{tabular}

Additional Signature may be generated within this form by selecting the Add button.

This collection of information is required by $37 \mathrm{CFR} 1.76$. The information is required to obtain or retain a benefit by the public which is to file (and by the USPTO to process) an application. Confidentiality is governed by 35 U.S.C. 122 and 37 CFR 1.14. This collection is estimated to take 23 minutes to complete, including gathering, preparing, and submitting the completed application data sheet form to the USPTO. Time will vary depending upon the individual case. Any comments on the amount of time you require to complete this form and/or suggestions for reducing this burden, should be sent to the Chief Information Officer, U.S. Patent and Trademark Office, U.S. Department of Commerce, P.O. Box 1450, Alexandria, VA 22313-1450. DO NOT SEND FEES OR

COMPLETED FORMS TO THIS ADDRESS. SEND TO: Commissioner for Patents, P.O. Box 1450, Alexandria, VA 22313-1450. 


\section{Privacy Act Statement}

The Privacy Act of 1974 (P.L. 93-579) requires that you be given certain information in connection with your submission of the attached form related to a patent application or patent. Accordingly, pursuant to the requirements of the Act, please be advised that: (1) the general authority for the collection of this information is 35 U.S.C. 2(b)(2); (2) furnishing of the information solicited is voluntary; and (3) the principal purpose for which the information is used by the U.S. Patent and Trademark Office is to process and/or examine your submission related to a patent application or patent. If you do not furnish the requested information, the U.S. Patent and Trademark Office may not be able to process and/or examine your submission, which may result in termination of proceedings or abandonment of the application or expiration of the patent.

The information provided by you in this form will be subject to the following routine uses:

1. The information on this form will be treated confidentially to the extent allowed under the Freedom of Information Act (5 U.S.C. 552$)$ and the Privacy Act (5 U.S.C. 552a). Records from this system of records may be disclosed to the Department of Justice to determine whether the Freedom of Information Act requires disclosure of these records.

2. A record from this system of records may be disclosed, as a routine use, in the course of presenting evidence to a court, magistrate, or administrative tribunal, including disclosures to opposing counsel in the course of settlement negotiations.

3. A record in this system of records may be disclosed, as a routine use, to a Member of Congress submitting a request involving an individual, to whom the record pertains, when the individual has requested assistance from the Member with respect to the subject matter of the record.

4. A record in this system of records may be disclosed, as a routine use, to a contractor of the Agency having need for the information in order to perform a contract. Recipients of information shall be required to comply with the requirements of the Privacy Act of 1974, as amended, pursuant to 5 U.S.C. $552 \mathrm{a}(\mathrm{m})$.

5. A record related to an International Application filed under the Patent Cooperation Treaty in this system of records may be disclosed as a routine use, to the International Bureau of the World Intellectual Property Organization, pursuant to the Patent Cooperation Treaty.

6. A record in this system of records may be disclosed, as a routine use, to another federal agency for purposes of National Security review (35 U.S.C. 181) and for review pursuant to the Atomic Energy Act (42 U.S.C. 218(c)).

7. A record from this system of records may be disclosed, as a routine use, to the Administrator, General Services, or his/her designee, during an inspection of records conducted by GSA as part of that agency's responsibility to recommend improvements in records management practices and programs, under authority of 44 U.S.C. 2904 and 2906 . Such disclosure shall be made in accordance with the GSA regulations governing inspection of records for this purpose, and any other relevant (i.e., GSA or Commerce) directive. Such disclosure shall not be used to make determinations about individuals.

8. A record from this system of records may be disclosed, as a routine use, to the public after either publication of the application pursuan to 35 U.S.C. 122(b) or issuance of a patent pursuant to 35 U.S.C. 151. Further, a record may be disclosed, subject to the limitations of 37 CFR 1.14, as a routine use, to the public if the record was filed in an application which became abandoned or in which the proceedings were terminated and which application is referenced by either a published application, an application open to public inspections or an issued patent.

9. A record from this system of records may be disclosed, as a routine use, to a Federal, State, or local law enforcement agency, if the USPTO becomes aware of a violation or potential violation of law or regulation. 


\begin{tabular}{|c|c|c|c|c|}
\hline \multicolumn{5}{|c|}{ Electronic Patent Application Fee Transmittal } \\
\hline \multicolumn{5}{|l|}{ Application Number: } \\
\hline \multicolumn{5}{|l|}{ Filing Date: } \\
\hline Title of Invention: & \multicolumn{4}{|c|}{ EMOTIONAL-GESTURE BASED COMMUNICATION METHOD } \\
\hline First Named Inventor/Applicant Name: & \multicolumn{4}{|l|}{ Afarin Pirzadeh } \\
\hline Filer: & \multicolumn{4}{|c|}{ Joel Alphonso Henry } \\
\hline Attorney Docket Number: & \multicolumn{4}{|c|}{ IURTC-2015-124-01-US-E } \\
\hline \multicolumn{5}{|l|}{ Filed as Small Entity } \\
\hline \multicolumn{5}{|l|}{ Filing Fees for Provisional } \\
\hline Description & Fee Code & Quantity & Amount & $\begin{array}{c}\text { Sub-Total in } \\
\text { USD(\$) }\end{array}$ \\
\hline \multicolumn{5}{|l|}{ Basic Filing: } \\
\hline Provisional Application Filing Fee & 2005 & 1 & 130 & 130 \\
\hline \multicolumn{5}{|l|}{ Pages: } \\
\hline \multicolumn{5}{|l|}{ Claims: } \\
\hline \multicolumn{5}{|l|}{ Miscellaneous-Filing: } \\
\hline \multicolumn{5}{|l|}{ Petition: } \\
\hline \multicolumn{5}{|l|}{ Patent-Appeals-and-Interference: } \\
\hline Post-Allowance-and-Post-Issuance: & & & & \\
\hline
\end{tabular}




\begin{tabular}{|c|c|c|c|c|}
\hline Description & Fee Code & Quantity & Amount & $\begin{array}{c}\text { Sub-Total in } \\
\text { USD(\$) }\end{array}$ \\
\hline \multicolumn{5}{|l|}{ Extension-of-Time: } \\
\hline \multicolumn{5}{|l|}{ Miscellaneous: } \\
\hline \multicolumn{4}{|c|}{ Total in USD (\$) } & 130 \\
\hline
\end{tabular}




\begin{tabular}{|c|c|}
\hline \multicolumn{2}{|c|}{ Electronic Acknowledgement Receipt } \\
\hline EFS ID: & 22111216 \\
\hline Application Number: & 62150069 \\
\hline \multicolumn{2}{|l|}{ International Application Number: } \\
\hline Confirmation Number: & 1192 \\
\hline Title of Invention: & EMOTIONAL-GESTURE BASED COMMUNICATION METHOD \\
\hline First Named Inventor/Applicant Name: & Afarin Pirzadeh \\
\hline Customer Number: & 43556 \\
\hline Filer: & Joel Alphonso Henry \\
\hline \multicolumn{2}{|l|}{ Filer Authorized By: } \\
\hline Attorney Docket Number: & IURTC-2015-124-01-US-E \\
\hline Receipt Date: & 20-APR-2015 \\
\hline \multicolumn{2}{|l|}{ Filing Date: } \\
\hline Time Stamp: & $17: 26: 20$ \\
\hline Application Type: & Provisional \\
\hline
\end{tabular}

Payment information:

\begin{tabular}{|l|l|}
\hline Submitted with Payment & yes \\
\hline Payment Type & Deposit Account \\
\hline Payment was successfully received in RAM & $\$ 130$ \\
\hline RAM confirmation Number & 4935 \\
\hline Deposit Account & 020390 \\
\hline Authorized User & \\
\hline $\begin{array}{l}\text { The Director of the USPTO is hereby authorized to charge indicated fees and credit any overpayment as follows: } \\
\text { Charge any Additional Fees required under 37 C.F.R. Section 1.16 (National application filing, search, and examination fees) }\end{array}$ \\
\hline
\end{tabular}




\begin{tabular}{|c|c|c|c|c|c|}
\hline \multicolumn{6}{|c|}{ File Listing: } \\
\hline $\begin{array}{c}\text { Document } \\
\text { Number }\end{array}$ & Document Description & File Name & $\begin{array}{l}\text { File Size(Bytes)/ } \\
\text { Message Digest }\end{array}$ & \begin{tabular}{c|c|} 
Multi \\
Part /.zip
\end{tabular} & $\begin{array}{c}\text { Pages } \\
\text { (if appl.) }\end{array}$ \\
\hline \multirow{2}{*}{1} & & \multirow{2}{*}{ IURTC-2015-124-01-APP.pdf } & 125918 & \multirow{2}{*}{ yes } & \multirow{2}{*}{7} \\
\hline & & & 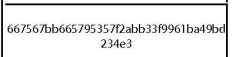 & & \\
\hline & \multicolumn{5}{|c|}{ Multipart Description/PDF files in .zip description } \\
\hline & \multicolumn{2}{|c|}{ Document Description } & Start & \multicolumn{2}{|c|}{ End } \\
\hline & \multicolumn{2}{|c|}{ Specification } & 1 & \multicolumn{2}{|c|}{5} \\
\hline & \multicolumn{2}{|c|}{ Claims } & 6 & \multicolumn{2}{|c|}{6} \\
\hline & \multicolumn{2}{|c|}{ Abstract } & 7 & \multicolumn{2}{|c|}{7} \\
\hline \multicolumn{6}{|l|}{ Warnings: } \\
\hline \multicolumn{6}{|l|}{ Information: } \\
\hline \multirow{2}{*}{2} & \multirow{2}{*}{$\begin{array}{l}\text { Drawings-only black and white line } \\
\text { drawings }\end{array}$} & \multirow{2}{*}{ IURTC-2015-124-01-DRAW.pdf } & 1234961 & \multirow{2}{*}{ no } & \multirow{2}{*}{21} \\
\hline & & & 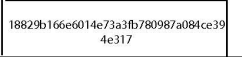 & & \\
\hline \multicolumn{6}{|l|}{ Warnings: } \\
\hline \multicolumn{6}{|c|}{$\begin{array}{l}\text { The page size in the PDF is too large. The pages should be } 8.5 \times 11 \text { or A4. If this PDF is submitted, the pages will be resized upon entry into the } \\
\text { Image File Wrapper and may affect subsequent processing }\end{array}$} \\
\hline \multicolumn{6}{|l|}{ Information: } \\
\hline \multirow[b]{2}{*}{3} & \multirow{2}{*}{ Application Data Sheet } & \multirow{2}{*}{ IURTC-2015-124-01-ADS.pdf } & 1503257 & \multirow{2}{*}{ no } & \multirow[b]{2}{*}{6} \\
\hline & & & 2783976762655 efledde96341049510a930e & & \\
\hline \multicolumn{6}{|l|}{ Warnings: } \\
\hline \multicolumn{6}{|l|}{ Information: } \\
\hline \multirow{2}{*}{4} & \multirow{2}{*}{ Fee Worksheet (SB06) } & \multirow{2}{*}{ fee-info.pdf } & 29802 & & \\
\hline & & & 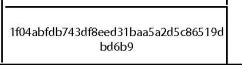 & no & 2 \\
\hline Warnings: & & & & & \\
\hline Information: & & & & & \\
\hline & & Total Files Size (in bytes) & & 93938 & \\
\hline
\end{tabular}


This Acknowledgement Receipt evidences receipt on the noted date by the USPTO of the indicated documents, characterized by the applicant, and including page counts, where applicable. It serves as evidence of receipt similar to a Post Card, as described in MPEP 503.

New Applications Under 35 U.S.C. 111

If a new application is being filed and the application includes the necessary components for a filing date (see 37 CFR 1.53(b)-(d) and MPEP 506), a Filing Receipt (37 CFR 1.54) will be issued in due course and the date shown on this Acknowledgement Receipt will establish the filing date of the application.

National Stage of an International Application under 35 U.S.C. 371

If a timely submission to enter the national stage of an international application is compliant with the conditions of 35 U.S.C. 371 and other applicable requirements a Form PCT/DO/EO/903 indicating acceptance of the application as a national stage submission under 35 U.S.C. 371 will be issued in addition to the Filing Receipt, in due course.

New International Application Filed with the USPTO as a Receiving Office

If a new international application is being filed and the international application includes the necessary components for an international filing date (see PCT Article 11 and MPEP 1810), a Notification of the International Application Number and of the International Filing Date (Form PCT/RO/105) will be issued in due course, subject to prescriptions concerning national security, and the date shown on this Acknowledgement Receipt will establish the international filing date of the application. 


\section{References}

Alm, C. O., Roth, D., \& Sproat, R. (2005). Emotions from text: machine learning for textbased emotion prediction. In Proceedings of the conference on Human Language Technology and Empirical Methods in Natural Language Processing (pp. 579-586).

Aronoff, J., Woike, B. A., \& Hyman, L. M. (1992). Which are the stimuli in facial displays of anger and happiness - Configurational bases of emotion recognition. Journal of Personality and Social Psychology, 62(6), 1050-1066.

Boonthanom, R. (2004). Computer-mediated communication of emotions: a lens model $\begin{array}{llll}\text { approach. } & \text { (Doctoral } & \text { dissertation) }\end{array}$ http://citeseerx.ist.psu.edu/viewdoc/download?doi=10.1.1.85.5320\&rep=rep1\&typ e=pdf.

Boonthanom,R. (2004). Computer-mediated communication of emotions: a lens model $\begin{array}{llll}\text { approach. } & \text { (Doctoral } & \text { dissertation) }\end{array}$ http://hdl.handle.net/123456789/398.

Brave S, Dahley D (1997) inTouch: a medium for haptic interpersonal communication. In: Proceedings of the ACM Conference on Human Factors in Computing Systems (CHI97),Atlanta, GA, 22-27 March 1997

Brunswik, E. (1956). Perception and the Representative Design of Psychological Experiments. Berkeley, CA: University of California Press.

Burgoon, J. K., Buller, D. B., \& Woodall, W. G. (1996). Nonverbal communication: The unspoken dialogue (2nd ed.). New York: The McGraw-Hill.

Burleson, Brant R. (2010), 'The nature of interpersonal communication: A messagecentered approach', in C. R. Berger, M. E. Roloff and D. Roskos-Ewoldsen (eds), The Handbook of Communication Science, 2nd ed., Thousand Oaks, CA: Sage, pp. 145-63. 
Cathcart R, Gumpert G. (1983). Mediated interpersonal communication: toward a new typology. Q. J. Speech 69:267-77

Chapanis, A., Ochsman, R., Parrish, R., \& Weeks, G. (1972). Studies in interactive communication I: The effects of four communication modes on the behavior of teams during cooperative problem solving. Human Factors, 14(6), 487-509.

Chapanis, A., Ochsman, R., Parrish R., \& Weeks, G. (1977). Studies in interactive communication II: The effects of four communication modes on the linguistic performance of teams during.

Culnan, M. J. \& M. L. Markus (1987). Information Technologies. In F. M. Jablin, L. L. Putnam, K. H. Roberts and L. W. Porter (Eds.), Handbook of Organizational Communication: An Interdisciplinary Perspective (pp. 421-443). Newbury Park, CA: Sage Publications.

Dennis, A. R.,\&Kinney, S. T. (1998, May). Testing media richness theory in the newmedia: The effect of cues, feedback and task equivocality. Information Systems Research, 9, 30-33.

Derks, D., Bos, A. R., and von Grumbkow, J. 2008. Emoticons in Computer-Mediated Communication: Social Motives and Social Context. Cyberpsychology \& Behavior, 11, 1 (2008), 99-101. DOI=10.1089/cpb.2007.9926

Derks, D., Fischer, A.H., Bos, A. (2008) The role of emotion in computer mediatedcommunication: A review. Journal of Computer in Human Behavior, 24(3), 766785.

Duncan, S., "Some signals and rules for taking speaking turns in conversations," Journal of Personality and Social Psychology, vol. 23, no. 2, pp. 283-292, 1972.

Ekman, P. 1982. Emotion in the human face. Cambridge University Press.

Ekman, Paul \& Friesen, W.V. (1975). Unmasking the Face. Prentice-Hall. 
Fridlund, A. J., Ekman, P., \& Oster, H. (1987). Facial Expressions of Emotion: Review of Literature, 1970-1983. In Siegman, A. W. \& Feldstein, S. (Eds.), Nonverbal Behavior and Communication (pp. 143-224). Hillsdale, NJ: Lawrence Erlbaum Associates.

Fussell, S. R. (2002). The verbal communication of emotion: Interdisciplinary perspectives: Introduction and overview. In S. R. Fussell, (Ed.) The verbal communication of emotion: Interdisciplinary perspectives. Mahwah, NJ: Lawrence Erlbaum Associates. Download Preprint (PDF)

Gajadhar, J., \& Green, J. (2005). The importance of nonverbal elements in online chat. Educause Quarterly, 63-64.

Garcia, A. \& Jacobs, J. (1999) The eyes of the beholder: Understanding the turn-taking system in quasi-synchronous computer mediated communication. Research on Language and Social Interaction, 32 (4). 337-367

Gasper, K. (2003). When necessity is the mother of invention: Mood and problem solving. Journal of Experimental Social Psychology, 39(3), 248-262.

Glaser, B., \& Strauss, A. (1967). The discovery of grounded theory: Strategies for qualitative research. Chicago: Aldine.

Goffman, E. (1972). Fun in games. In Goffman E. (Eds.), Encounters (pp. 5-72). New York: Penguin.

Goldberg, L. R., Johnson, J. A., Eber, H. W., Hogan, R., Ashton, M. C., Cloniger, C. R., \& Gough, H. G. (2006). The international personality item pool and the future of public-domain personality measures. Journal of Research in Personality, 40, 8496.

Grinter, R.E. and M.A. Eldrige. (2001). Y do tngrs luv 2 txt msg? In Proceedings of the Seventh European Conference on Computer-Supported Cooperative Work 
ECSCW '01 Bonn, Germany. W. Prinz, M.Jarke, Y. Rogers, K. Schmidt and V. Wulf (eds), 219-238.

Hancock, J.T. 2004. Verbal irony use in computer-mediated and face-to-face conversations. Journal of Language and Social Psychology, 23, 447-463.

Hancock, J. T., Landrigan, C., \& Silver, C. (2007). Expressing emotion in text-based communication. Proceedings of SIGCHI' 07: ACM Conference on Human factors in computing systems, 929-932.

Hancock, J. T., Gee, K., Ciaccio, K., \& Lin, J. M. H. (2008). I'm sad you're sad: Emotional contagion in CMC. Proceedings of CSCW '08: ACM Conference on Computer Supported Cooperative Work, 295-298.

Harrison, R. P. (1974). Beyond words. Englewood Cliffs, NJ: Prentice Hall.

Helton, W. S., \& Garland, G. (2006). Short stress state questionnaire: Relationships with reading comprehension and land navigation. In Proceedings of the 50th Annual Meeting of the Human Factors and Ergonomics Society (pp. 1731-1735).

Iannizzotto, G., Villari, M. \& Vita, L. (2001) Hand tracking for human-computer interaction with graylevel visualglove: Turning back to the simple way. In: Workshop on Perceptive User Interfaces. ACM Digital Library. ISBN 1-58113-448.

Isaacs, E., Whittaker, S., Frohlich, F., and O'Conaill, B. (1997). Informal communication reexamined: New functions for video in supporting opportunistic encounters". In K. Finn, A. Sellen, and S. Wilbur, Eds., Video-mediated communication, pps 459486. Mahwah, NJ: Lawrence Erlbaum Associates,1997.

Johnstone, T., \& Scherer, K.R. (2000). Vocal communication of emotion. In M. Lewis \& J. Haviland, (Eds.), Handbook of emotion (2nd ed., pp. 220-235). New York, NY: Guilford Press. 
Kaliouby, R. E., \& Robinson, P. (2004). FAIM: integrating automated facial affect analysis in instant messaging. Paper presented at the Proceedings of the 9th international conference on Intelligent user interfaces.

Karam, M., Schraefel, M. (2005). A Taxonomy of Gestures in Human Computer Interaction. Technical Report ECSTR-IAM05-009, Electronics and Computer Science, University of Southampton.

Kiesler, S., Siegel, J., \& McGuire, T. (1984). Social psychological aspects of computermediated communication. American Psychologist, 39, 1123-1134.

Kleinke, C. (1986). Gaze and eye contact: a research review. Psychological Bulletin, 100, 78-100.

Krippendorf, K. (1980). Content analysis: An introduction to its methodology. Beverly Hills, CA: Sage.

Lefcourt, H. M. (1981). The construction and development of the multidimensionalmultiattributional causality scales. In H. M. Lefcourt (Ed.), Research with the locus of control construct (Vol. 1: Assessment methods, pp. 245- 261). New York: Academic Press.

Lo, O. (2006). The roles of gratification opportunities, gratifications-obtained, and demographics in determining usage preference of instant messaging and e-mail among college students. Unpublished thesis, School of Journalism \& Communication, The Chinese University of Hong Kong.

Marteau, T. M., \& Bekker, H. (1992). The development of a six-item short-form of the state scale of the Spielberger State-Trait Anxiety Inventory (STAI). The British Journal of Clinical Psychology, 31(3), 301-306.

Mazeland, H. (2006). Conversation analysis. In Brown, K. (Eds.), Encyclopedia of language and linguistics, Vol. 3 (2nd ed.) (pp. 153-162). Oxford \& Amsterdam: Elsevier. 
McNeese, M. D., Bains, P., Brewer, I., Brown, C., Connors, E. S., Jefferson, T., . . . Terrell, I. S. (2005). The NeoCITIES simulation: Understanding the design and experimental methodology used to develop a team emergency management simulation. In Proceedings of the 49th Annual Meeting of the Human Factors and Ergonomics Society (pp. 591-594).

Mehrabian, A. (1972), Nonverbal commmunication. Chicago: Aldine-Atherton.

Morris, W. N. 1989. Mood: The Frame of Mind. New York: Springer-Verlag.

Nardi, B. 2005. Beyond bandwidth: Dimensions of connection in interpersonal communication. Computer Supported Cooperative Work, 14, 2(2005), 91-130.

Nelson, H.G. and Stoltermann, E. The Design Way: intentional change in an unpredictable world . Educational Technology Publications, Englewood Cliffs, NJ, 2003.

Neviarouskaya, Alena, Helmut Prendinger, and Mitsuru Ishizuka. "User study of AffectIM, an emotionally intelligent instant messaging system." Intelligent Virtual Agents. Springer Berlin Heidelberg, 2008.

Oakley, I. O. M., S. (2003). Contact IM: Exploring asynchronous touch over distance. Paper presented at the CSCW'02.

Oviatt S, and Cohen P. (1991) Discourse Structure and Performance Efficiency in Interactive and non-Interactive Spoken Modalities. Computer Speech and Language, 5, 297-326.

Parkinson B, Fischer AH, and Manstead ASR. 2005. Emotions in social relations: cultural, group and interpersonal processes. New York: Psychology Press.

Pang, B., and Lee, L. 2008. Opinion Mining and Sentiment Analysis. Now Publishers Inc. Petrides, K., Pita, R., \& Kokkinaki, F. 2007. The location of trait emotional intelligence in personality factor space. British Journal of Psychology. 98, 273-289. 
Pfaff, M. S. (2012). Negative affect reduces team awareness: The effects of mood and stress on computer-mediated team communication. Human Factors, 54(4), 560571.

Pfaff, M. S., \& McNeese, M. D. (2010). Effects of mood and stress on distributed team cognition. Theoretical Issues in Ergonomics Science, 11(4), 321-339.

Pirzadeh, A., and Pfaff, M. S. 2012. Emotion expression under stress in instant messaging. Proceedings of the Human Factors and Ergonomics Society. 493497.

Planalp, S. (1998). Current issues arising at the confluence of communication and emotion. Australian Journal of Communication, 25, 65-70.

Ramirez, A., \& Broneck, K. (2009). 'IM me': Instant messaging as relational maintenance and everyday communication. Journal of Social and Personal Relationships, 26, 291-314.

Reid, A. (1977). Comparing the telephone with face-to-face interaction. In I. Pool Ed., The Social Impact of the Telephone (pp. 386-414). Cambridge, MA: MIT Press.

Riva, G. (2002). The sociocognitive psychology of computer-mediated communication: The present and future of technology-based interactions. CyberPsychology \& Behavior, 5, 581-598.

Rottenberg, J., and Ray, R. D., \& Gross, J. J. 2007. Emotion elicitation using films. In J. A. Coan \& J. J. B. Allen (Eds.), The handbook of emotion elicitation and assessment. London: Oxford University Press.

Rovers, A. F., and Harm A. van Essen. "HIM: a framework for haptic instant messaging." CHI'04 Extended Abstracts on Human Factors in Computing Systems. ACM, 2004.

Russell, J. A. 1980. A circumplex model of affect. Journal of Personality and Social Psychology. 39, 1161-1178. 
Sacks, H., Schegloff, E. A., \& Jefferson, G. (1974). A simplest systematics for the organization of turn-taking for conversation. Language, 50 (4), 696-735.

Sasaki, M. U., \& Ohbuchi, K. (1999). Conflict processes on computer-mediated communication. Tohoku Psychologica Folia, 58, 50-55.

Sawada, M., Suda, K., \& Ishii, M. (2003). Expression of emotions in dance: relation between arm movement characteristics and emotion. Perceptual and Motor Skills, $97,697-708$.

Schegloff, E. A. (1987). Analyzing Single Episodes of Interaction: An Exercise in Conversation Analysis. Social Psychology Quarterly, 50(2), 101-114.

Schegloff, E. A., Koshik, I., Jacoby, S., \& Olsher, D. (2002). Conversation analysis and applied linguistics. Annual Review of Applied Linguistics, 22, 3-31.

Scherer, K.R. (1978). Personality inference from voice quality: The loud voice of extroversion. European Journal of Social Psychology, 8, 467-487.

Scherer, K. R. (2003). Vocal communication of emotion: A review of research paradigms. Speech Communication, 40, 227-256.

Scherer, K. R. (2005). What are emotions? And how can they be measured? Social Science Information, 44(4), 695- 729.

Sexton, J. B., \& Helmreich, R. L. (2000). Analyzing cockpit communication: The links between language, performance, error, and workload. Human Performance in Extreme Environments, 5(1), 63-68.

Shannon, C. E., \& Weaver, W. (1949). The Mathematical Theory of Information. Champaign, IL: University of Illinois Press.

Short, J, Williams E, \& Christie, B. (1976). The social psychology of telecommunications. London: Wiley Press.

Sidnell, J. and Stivers, Tanya. (eds.) 2012. The Handbook of Conversation Analysis. Malden, MA: Wiley-Blackwell. 
Spector, P. E. (1982). Behavior in organizations as a function of employee's locus of control. Psychological Bulletin, 91(3), 482-497.

Spielberger, C. D. (1983). Manual of the State-Trait Anxiety Inventory, form Y. Palo Alto, CA: Consulting Psychologists Press.

Streeck, J., \& Knapp, M. L. (1992). The interaction of visual and verbal features in human communication (. In F. Poyatos (Ed.), Advances in Nonverbal Communication (pp. 3-24). Amsterdam/Philadelphia: John Benjamins Publishing Co.

Tagliamonte, A., \& Denis, D. (2008). Linguistic ruin? LOL! Instant messanging and teen language. American Speech, 83.

Tausczik, Y. R., \& Pennebaker, J. W. (2010). The psychological meaning of words: LIWC and computerized text analysis methods. Journal of Language and Social Psychology, 29(1), 24-54.

Wagner, H. L., and Smith, J. 1991. Facial expression in the presence of friends and strangers. Journal of Nonverbal Behavior. 15, 201-214.

Walther, J.B. (1992). Interpersonal effects in computer-mediated interaction: A relational perspective. Communication Research, 19, 52-60.

Walther, J. B. (1993). Impression development in computer-mediated interaction. Western Journal of Communication, 57, 381-398.

Walther, J. B., Anderson, J. F., \& Park, D. W. (1994). Interpersonal effects in computermediated interaction: A meta-analysis of social and antisocial communication. Communication Research, 21(4), 460-487.

Walther JB. (1996). Computer-mediated communication: Impersonal, interpersonal and hyperpersonal interaction. Communication Research. 23(1), 3-43. 
Walther, J. B., \& D'Addario, K. P. (2001). The impacts of emoticons on message interpretation in computer-mediated communication. Social Science Computer Review, 19, 323-345.

Walther , J.B., Loh, T., \& Granka, L. (2005) Let me count the ways: The interchange of verbal and nonverbal cues in computermediated and face-to-face affinity. Journal of Language and Social Psychology, 24, 36-65.

Wang, H., Prendinger, H., \& Igarashi, T. (2004). Communicating emotions in online chat using physiological sensors and animated text. Paper presented at the $\mathrm{CHI}$ '04 extended abstracts on Human factors in computing systems.

Watson, D., Clark, L. A., \& Tellegen, A. (1988). Development and validation of brief measures of positive and negative affect: The PANAS scales. Journal of Personality and Social Psychology, 54(6), 1063-1070.

Watt, R. (1995). An Examination Of The Visual Aspects Of Human Facial Gesture. In S. Emmot (Ed.), Information superhighways: multimedia users and futures. London, Academic Press.

Westermann R, Spies K, Stahl G, Hesse FW. 1996. Relative effectiveness and validity of mood induction procedures: a meta- analysis. Eur. J. Soc. Psychol. 26, 557-580

Whittaker, S. (2003). Theories and Methods in Mediated Communication. In A. Graesser (ed.): The Handbook of Discourse Processes. Cambridge: Hillsdale NJ: Lawrence Erlbaum.

Yeo, Z. (2008). Emotional instant messaging with KIM. Paper presented at the CHI '08 extended abstracts on Human factors in computing systems.

Zimmerman, J., Forlizzi, J., \& Evenson, S. (2007). Research through design as a method for interaction design research in $\mathrm{HCl}$. In Proc. CHI '07 , 493-502. 
Curriculum Vitae

\section{Afarin Pirzadeh}

\section{Education}

IUPUI, School of Informatics and Computing, Indianapolis, IN

PhD in Human-Computer Interaction (GPA 3.94)

May 2016

Kish University, Humanities Department, Kish, Iran

Sept 2009

Master of Business Administration (GPA 18.10/20)

Amirkabir University,Computer Engineering Department,Tehran, Iran

Sept 2009

B.Sc. in Information Technology (GPA 16.24/20)

\section{Computing skill set}

- Database Management: MS SQL Server 2005, MS-Access

- Operating Systems: Windows XP, Windows Vista

- Programming Languages: C, PowerBuilder, C++, Java, HTML, SQL

- Software Packages: SPSS, R

\section{Human-computer interaction $(\mathrm{HCl})$ skill set}

$\mathrm{HCl}$ Skills: Quantitative and qualitative user research, Wire-framing, contextual inquiry, ethnography, Interviews, personas and story telling/scenarios, role playing,

survey/questionnaire, affinity diagramming, story boarding and journey maps, work flow modeling, information architecture, conceptual mapping, user experience design, participatory design and ideation, , reframing, prototyping, usability evaluation, user testing, walkthrough, heuristic evaluation, A/B testing, conversation analysis, Cognitive walkthrough, heuristic evaluation, website analytics, SWOT analysis, competitive analysis, business process modeling.

$\mathrm{HCl}$ tools: PowerPoint, OmniGraffle, Keynote, Balsamiq, Axure, Sketch Flow, Photoshop, Illustration, Morae Recorder and Manager, Camtasia

\section{Patent}

Emotional-Gesture Based Communication Method Date filed: 04/20/2015. Inventor: Afarin Pirzadeh.

US Provisional Patent Application No. 62/150,069

\section{Employment history}

Formstack, Indianapolis, US

Nov 2015 - Feb 2016

UX Researcher (Internship)

- Conducting user research (usability testing, $A / B$ testing, and interviews) to improve current products and developing new features/products based on users need

IUPUI, Indianapolis, IN

Aug 2010 - Aug 2014

Research Assistant - School of Informatics and Computing

Assisting Dr. Mark Pfaff (PI) in different projects at GRAPPA (Group Psychology and Performance Lab)

- Literature review on diverse topics of distributed teamwork, information sharing, and individual and collaborative decision making 
- Brainstorming on MITRE project on supporting complex decision making through option awareness

- Conducting usability evaluation

Intuit, San Diego, US

UX Designer/Researcher (Internship)

May 2014 - Aug 2014

- Conducted heuristic evaluation on TurboTax-healthcare website and proposed redesign solutions

- Developed low fidelity prototype, using keynote, for part of the tax-exempt application process

Xerox Research Center Webster (XRCW), Webster

May 2013 - Aug 2013

UX Researcher/Designer (Internship)

- Developed and documented a framework for Ignite, Educators Support System

- Developed the design conceptual model for different components of Ignite

- Conducted the design process and developed a low fidelity prototype of "Essay" type of assessment, selected from the conceptual model, from both teachers and students points of view

Tosan, Banking Solution Provider, Iran

May 2009 - July 2010

UX lead (Aug 2009 - July 2010)

- Conducted heuristic evaluation for two products of the company, Internet and mobile banking applications

- Interviewed customers and stakeholders to identify their expectations and systems problems

- Organized meetings prioritized issues of usability evaluation, developed electronic prototype and presented to development team for implementation. Developed paper prototype and conducted usability test

Consultant (May 2009 - Aug 2009)

- Analyzed the work flow of testing in the of software development process

- Improved test processes by TPI (Test Process Improvement) model, resulting in better documentation of software bugs and time savings

Avajang, Hardware Service Company, Tehran, Iran $\quad$ Dec 2008 - March 2009 Business Analyst

- Developed Business Process Model by conducting contextual inquiry

- Identified breakdowns of different work models

- Presented solutions for the business process improvement to the management board which when implemented, reduced lead time of process and resulted in cost savings and higher employees satisfaction

Parsun Farayand, Computer Company, Iran Jan 2007 - Jan 2008

IT Project Manager

- Planned, prepared and tracked immediate reports for the managerial board of the APC (Automated Passenger Check-in) project

- Supervised six team members

Data Processing, Computer Company, Iran

July 2005 - Oct 2005

System Analyst (Internship) 
- Conducted requirement analysis of work force management system as one part of Iran Switch Management Network project. Application was to automate assigning different troubles to work force and dispatch them to the location

\section{Research/design projects experience}

IUPUI, Indianapolis, IN, US (Ph.D. Dissertation) Aug 2011 - May 2016

Emotional communication in instant messaging- Advisor: Dr. Mark Pfaff

- Conducted user research both quantitative (questionnaires, content analysis) and qualitative (e.g. conversation analysis, interviews, brainstorming sessions, and participatory design sessions) to examine the strategies users apply to overcome the lack of visual and aural nonverbal communication in instant messaging

- Conducted user-centered design process and established multi-touch gesture based design strategies to enhance nonverbal communication in this text messaging

- Designed and evaluated paper prototyping of Gestchat, a text messaging mobile application to support emotional communication

- Accomplishment: Five research publications, low-fidelity prototype of Gestchat, an instant messaging tool to support emotional communication

IUPUI, Indianapolis, IN, US (Ph.D. project)

Aug 2012 - Oct 2015

Personal informatics and reflection: a critical examination of the nature of reflectionAdvisor: Dr. Erik Stolterman

- Conducted user research to examine the intrapersonal communication in the context of intimate relationships

- Conducted user-centered design process and established design strategies to support reflection, meaning making, and learning

- Developed and evaluated paper prototyping of Inome, a mobile application to support young women to reflect on their dating experience with the purpose of improving self-identity

- Accomplishment: Two research publications, low-fidelity prototype of Inome, a journaling tool to support reflection and learning

IUPUI, Indianapolis, IN, US (Class projects)

Priceline.com - Usability and Evaluative Methods 1543

Graduate Student in $\mathrm{HCl}$

- Conducted scenario-based and heuristics evaluation inspection on the usability of priceline.com website

- Reported the usability problems and recommended strategies for improvements

Priceline.com - Usability and Evaluative Methods 154

Graduate Student in $\mathrm{HCl}$

- Conducted user testing with 10 participants across 5 tasks

- Reported success rate, time on task and other findings tasks by tasks

Recommended strategies for improvements based on the user study findings

Bamboo - HCl1 1541

Graduate Student in $\mathrm{HCl}$

- Designed Bamboo, a mobile device could be used by doctors in a hospital, which integrates all of their workday needs to increase their effectiveness, reduce errors, and increase patients' satisfaction 
- Conducted the design evaluation with interviewing the users and the internal walkthrough

- Revised the interactive prototype based on the usability study findings

On Set - HCl1 I541

Graduate Student in $\mathrm{HCl}$

- Designed On Set, a tool to provide a space for communication where all members of the crew can actively participate in the filmmaking process from the first steps of pre-production to the final stages of post-production

- Sketched the paper prototype of On Set

- Conducted the design evaluation with interviewing the users and the cognitive walkthrough

- Reported the usability issues and revised the interactive prototype based on these findings

SmartShopper - HCl2 1561

Graduate Student in $\mathrm{HCl}$

- Designed SmartShopper, an on-cart tablet application that allows shoppers to quickly and visually compare key product features across brands based on their price, volume and nutritional values.

- Conducted contextual inquiry

- Consolidated all the information from the previous work to build several work and activity models

- Sketched the low-fidelity prototype based on the synthesized data

- Conducted internal walkthrough

ChaCha Powered by People - HCl2 1561

Graduate Student in $\mathrm{HCl}$

- Conducted a two-week diary study with 8 participants on existing ChaCha iPhone application

- Developed a high-fidelity prototype based on the collected users' feedbacks

Kish University, Kish, Iran (MBA Dissertation)

Studying the relationship between supervisors' emotional intelligence and their subordinates' styles of conflict management in small and medium enterprises Advisor:

Dr. Babak Alavi

- Measured emotional intelligence of supervisors and conflict management style of their subordinates in five SMEs in Tehran-Iran, using questionnaires

- Analyzed the gathered data and examined the main hypothesis of the study

\section{Entrepreneurial experience}

Gestchat, Indianapolis, IN, US

Aug 2014 - Present

Founder and Project lead

Gestchat is text messaging mobile application with unique features to support emotional communication gestchat.com

- Built a team of one programmer, two visual designers, one marketer, and one content writer and communicated design ideas of Gestchat with the purpose of market analysis and implementation of the minimum viable product

- Managed project timeline and team

- Conducted user testing to evaluate the minimum viable product 
- Redesigned Gestchat based on the user evaluation results

- Accomplishment: Implementation of minimum viable product and proof of concept, commercial video, full requirement analysis and design of next version of Gestchat

Inome, Indianapolis, IN, US

Aug 2014 - Present

Founder and Project lead

Inome is a tool that assists young women with reflecting on their dating relationship and supporting them to discover self-identity and improve personal growth, and positive selfesteem

- Built a team of two programmers and two visual designer and communicated the design ideas with them with the purpose of implementation of the application

- Conducted user testing to evaluate the minimum viable product

- Redesigned the product based on user evaluation

- Managed project timeline and team

- Redesigned Inome based on the user evaluation results

- Accomplishment: Implementation of minimum viable product and proof of concept, commercial video, full requirement analysis and design of the next version of Inome

\section{Teaching experience}

Teaching Assistant, Digital Story Telling (NEWM -N202), Fall 2015

Teaching Assistant, Digital Imagery (NEWM- N102), Fall 2015

Teaching Assistant, Seeing Sideways (NEWM- N385), Fall 2015

Teaching Assistant, School of Informatics Capstone, Fall 2015

Teaching Assistant, Informatics Research Design (INFO-I575), Spring 2015

Teaching Assistant, Interactive Design (NEWM- N285), Spring 2015

Teaching Assistant, Introduction to Human-Computer Interaction Practice (INFO-I270), Spring 2015

Teaching Assistant, Informatics Research Design (INFO-I575), Fall 2014

Teaching Assistant, Interaction Design Practice (INFO-H541), Fall 2014

Teaching Assistant, School of Informatics Capstone, Fall 2014

\section{Refereed Publications}

Afarin Pirzadeh, Mark Pfaff, S., (2014), " How do you IM when you get emotional" In Proc. Group Conference 2014.

Afarin Pirzadeh, Hsiao-Wen Wu, Reecha Bharali, Bomi Kim, Terri Wada, Mark Pfaff, S., (2014), "Designing Multi-Touch Gestures to Support Emotional Expression in IM", work in progress, CHI2014, Toronto.

Afarin Pirzadeh, Reecha Bharali, Terri Wada (2013), “ My Mirror-A Tool to Support Reflection” In Proc. HCl international Conference 2013.

Afarin Pirzadeh, Li He, Erik Stolterman, (2013), "Personal Informatics and Reflection: A Critical Examination of the Nature of Reflection", In Proc. alt.chi 2013.

Moon, Sung Pil; Liu, Yikun; Entezari, Steven O.; Pirzadeh, Afarin; Pappas, Andrew; Pfaff, Mark S., (2013), "Top Health Trends: An information visualization tool for awareness of local health trends", ISCRAM 2013. 
Afarin Pirzadeh, and Mark S. Pfaff, (2012), "Emotional expression under stress in instant messaging", In Proc. HFES'12.

Afarin Pirzadeh, and Mark S. Pfaff, (2012), "Expression of Emotion in Instant Messaging", In Proc. CSCW2012.

Preethi Srinivas, Huang Hai Dan, Afarin Pirzadeh, and Davide Bolchini, (2011), "Clever Shopper: Supporting In-Store Decision-Making", In Proc. Int. Conference on Pervasive Computing 2011.

Pirzadeh A., and Alavi S. B., (2011), "A study of relationship between managers' emotional intelligence and their staff's conflict management style", Journal of Management Sciences.

\section{Non-Refereed Publications and Posters}

Afarin Pirzadeh, and Mark Pfaff, S., "Emotional Communication in Instant Messaging", poster presented at the GROUP 2014, Sanibel Island, Florida.

Afarin Pirzadeh, Li He, Erik Stolterman, "Wandering Mind: A Tool to Support Reflection", poster presented at the Interaction 2013, Toronto.

Afarin Pirzadeh, Reecha Bharali, Terri Wada, " My Mirror-A Tool to Support Reflection", poster presented at $\mathrm{HCl}$ international Conference, Las Vegas (NV), July 2013.

Afarin Pirzadeh, Li He, Erik Stolterman, S., "Wandering Mind-A Tool to Support SelfReflection", poster presented at the 2013 Grace Hopper Celebration of Women in Computing (GHC), Minneapolis (Minnesota), Oct 2-5, 2013

Afarin Pirzadeh, and Mark Pfaff, S., "Expression of Emotion under Stress in Instant Messaging", poster presented at the 2012 IUPUI Research Day, Student Showcase, IUPUI Campus Center, Indianapolis (IN), April 13, 2012.

Afarin Pirzadeh, and Mark Pfaff, S., Pfaff, "Expression of Emotion in Instant Messaging", poster presented at the 2012 Women in Technology, IUPUI Campus Center, Indianapolis, April 11, 2012.

Afarin Pirzadeh, and Mark Pfaff, S., "Expression of Emotion in Instant Messaging", poster presented at the 2012 Grace Hopper Celebration of Women in Computing (GHC), Baltimore Convention Center, Baltimore (MD), October 3-6, 2012.

Afarin Pirzadeh, and Mark Pfaff, S., "Expression of Emotion in Instant Messaging", poster presented at the 2011 CRA-W Grad Cohort Workshop, Boston, Jan 15, 2011.

\section{Journal Article in Review}

Afarin Pirzadeh, \& Mark Pfaff, S., Emotional expression and response in instant messaging, The Journal of Collaborative Computing and Work Practices. (In review) 


\section{Doctoral Consortium Papers}

Afarin Pirzadeh, and Mark Pfaff, S., Emotional Communication in Instant Messaging, Accepted abstract at Richard Tapia Celebration of Diversity in Computing Conference, February 7, 2013.

Afarin Pirzadeh, and Mark Pfaff, S., Emotional Communication in Instant Messaging, Accepted abstract at GROUP Conference, November 9, 2014.

\section{Invited Presentations and Talks}

How do you IM when you get emotional, 2014 ACM Conference on Supporting Groupwork, (GROUP 2014), Sanibel Island (Florida), Nov 9-12, 2014.

Emotional expression under stress in instant messaging, 2012 Symposium on Human Factors and Ergonomics in Health Care: Bridging the Gap, (HFES'12), Baltimore (Maryland), March 12-14, 2012.

Emotional communication in IM, Guest Speaker at Informatics research design, (INFO1575), November, 2015

Emotional communication in IM, Guest Speaker at Interaction Design Practice, (INFOH541), November, 2014

\section{Honors and Awards}

IUPUI Innovation-To-Enterprise Award

Aug, 2015

Gestchat project got funded ( $\$ 14000$ ) by Innovation to Enterprise Central (ITEC)

ISSEC 2015 Student Pitch Competition Finalist

March 6, 2015

Selected as IUPUI Ideas Solving Social and Economic Challenges (ISSEC) 2015

Student Pitch Competition Finalist to present Gestchat idea

Richard Tapia Travel Scholarship to attend and present

Feb 2013, 2014

At Richard Tapia Celebration of Diversity in Computing Conference

Graduate Research Assistantship

Aug 2010 - Dec 2015

Indiana University Purdue University Indianapolis

\section{Service Activities}

Reviewer for Conference Papers

Aug 2010 - Aug 2015

- CHI'16 ACM Conference on Human Factors in Computing Systems - 2015

- CHI'15 ACM Conference on Human Factors in Computing Systems - 2014

\section{Languages}

English, Persian (Farsi), German

\section{Volunteer Work}

$\mathrm{HCl}$ International Conference, Las Vegas, NV

CHI'12 Conference, Austin, TX

July 2013 Student Volunteer

Computer Supported Cooperative Work (CSCW), Seattle

$\mathrm{HCl}$ International Conference, Orlando, FL

May 2012 Student Volunteer

Feb 2012 Student Volunteer

Int. Conference on Pervasive Computing, San Francisco

July 2011 Student Volunteer Jun 2011 Student Volunteer 